

\title{
Opbrengsttabellen Nederland 2018
}

\author{
Hans Jansen en Anne Oosterbaan \\ (Redactie)
}

Forest Ecology and Forest Management group, Wageningen University, Department of Environmental Sciences

Wageningen Environmental Research (WENR)
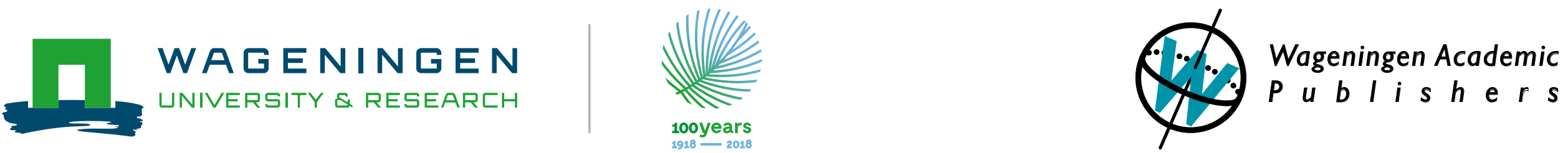
Behoudens de in of krachtens de Auteurswet van 1912 gestelde uitzonderingen mag niets uit deze uitgave worden verveelvoudigd, opgeslagen in een geautomatiseerd gegevensbestand, of openbaar gemaakt, in enige vorm of op enige wijze, hetzij elektronisch, mechanisch, door fotokopieën, opnamen of enige andere manier, zonder voorafgaande schriftelijke toestemming van de uitgever:

Wageningen Academic Publishers

Postbus 220, 6700 AE Wageningen

telefoon (0317) 476516

telefax (0317) 453417

www.WageningenAcademic.com

copyright@WageningenAcademic.com

e-EAN: 9789086868766

e-ISBN: 978-90-8686-876-6 DOI: $10.3920 / 978-90-8686-876-6$

\section{Omslagfoto's: Anne Oosterbaan}

Eerste uitgave, 2018

(C) Wageningen Academic Publishers Nederland, 2018
Voorzover het maken van reprografische verveelvoudigingen uit deze uitgave is toegestaan op grond van artikel $16 \mathrm{~h}$ Auteurswet 1912 dient men de daarvoor wettelijk verschuldigde vergoedingen te voldoen aan de Stichting Reprorecht (Postbus 3060, 2130 KB Hoofddorp, www.reprorecht.nl).

Voor het overnemen van gedeelte(n) uit deze uitgave in bloemlezingen, readers en andere compilatiewerken (artikel 16 Auteurswet 1912) kan men zich wenden tot de Stichting PRO (Stichting Publicatie- en Reproductierechten Organisatie, Postbus 3060, 2130 KB Hoofddorp, www.stichting-pro.nl).

De inhoud van deze publicatie en alle verantwoordelijkheden die daaruit voortkomen blijven de verantwoordelijkheid van de auteurs.

De uitgever aanvaardt geen aansprakelijkheid voor eventuele schade, die zou kunnen voortvloeien uit enige fout die in deze publicatie zou kunnen voorkomen. 
Deze publicatie is mogelijk gemaakt door financiële steun van

Staatsbosbeheer (www.staatsbosbeheer.nl), Unie van Bosgroepen (https://bosgroepen.nl), Probos (www.probos.nl) en Koninklijke Nederlandse Bosbouw Vereniging (http://knbv.nl).
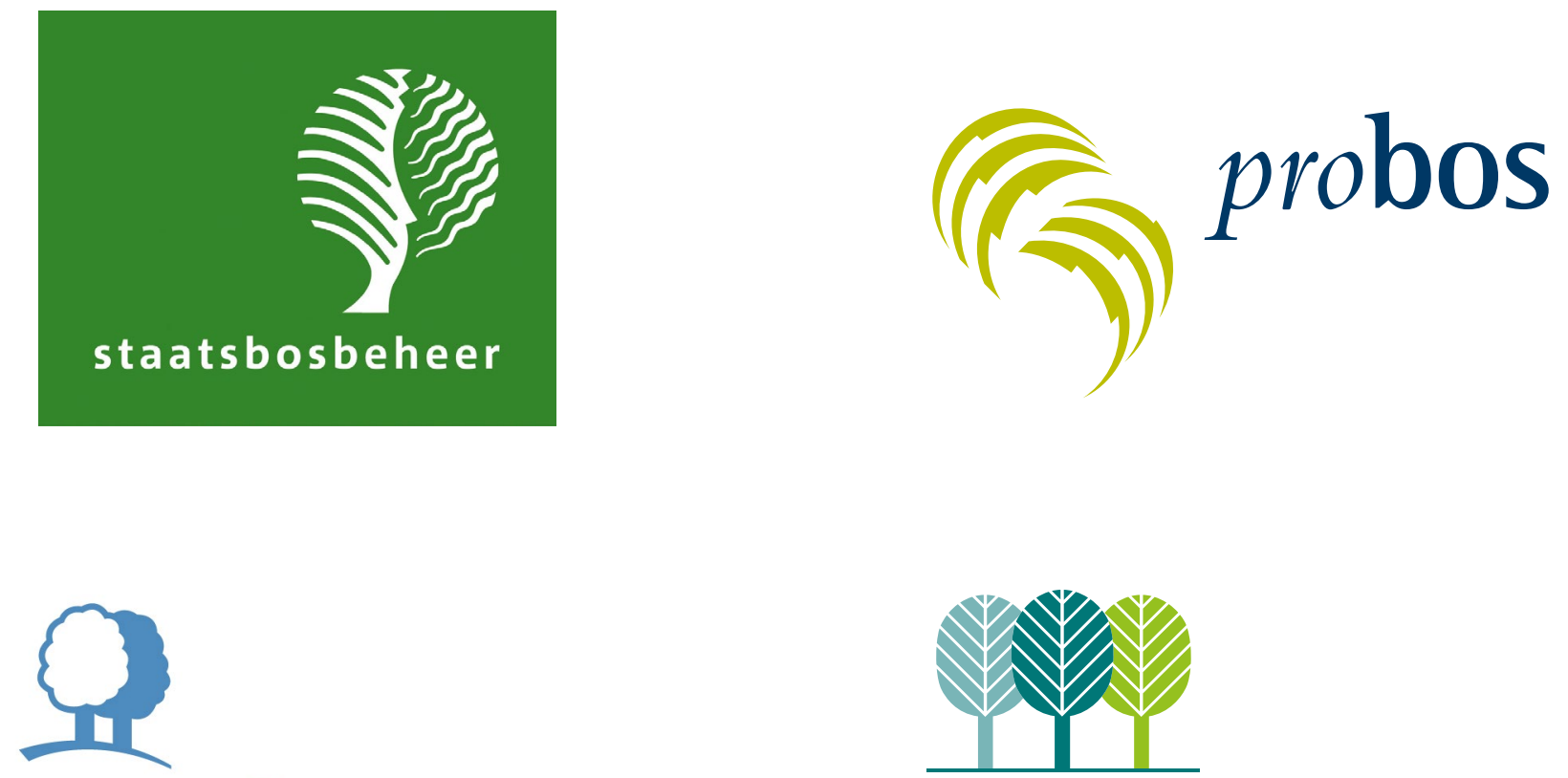

Unie van Bosgroepen

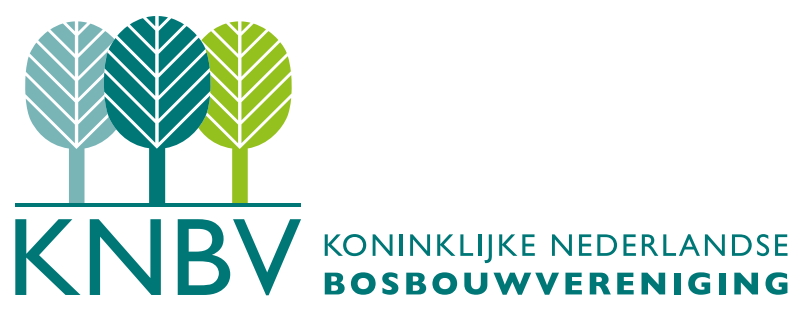




\section{J.J. Jansen en A. Oosterbaan (redactie), 2018. Opbrengsttabellen Nederland 2018, 172 blz.}

Met de volgende bijdragen:

Grove den door J.J. Jansen, G.M.J. Mohren, A. Oosterbaan, L. Goudzwaard, en J. den Ouden

Corsicaanse den door J.J. Jansen, A. Oosterbaan, G.M.J. Mohren en J. den Ouden

Oostenrijkse den door J.J. Jansen, A. Oosterbaan, G.M.J. Mohren en J. den Ouden

Douglas door J.J. Jansen, H. Schoonderwoerd, G.M.J. Mohren, E.A.H. Thomassen en J. den Ouden

Japanse lariks door J.J. Jansen, A. Oosterbaan, G.M. J. Mohren en J. den Ouden

Fijnspar door J.J. Jansen, G.M.J. Mohren, A. Oosterbaan en J. den Ouden

Zomereik door J.J. Jansen, A. Oosterbaan, G.M.J. Mohren en J. den Ouden

Amerikaanse eik door J.J. Jansen, A. Oosterbaan, G.M.J. Mohren en J. den Ouden

Beuk door J.J. Jansen, G.M.J. Mohren, A. Oosterbaan, L. Goudzwaard en J. den Ouden

Populier door J.J. Jansen, G.M.J. Mohren, P. Schmidt, L. Goudzwaard, A. Oosterbaan en J. den Ouden

Trilpopulier door J.J. Jansen, G.M.J. Mohren, P. Schmidt, L. Goudzwaard, A. Oosterbaan en J. den Ouden

Ruwe berk door J.J. Jansen, A. Oosterbaan, G.M. Mohren en J. den Ouden

Es door J.J. Jansen L. Goudzwaard, A. Oosterbaan, G.M.J. Mohren en J. den Ouden

Zwarte els door J.J. Jansen, A. Oosterbaan, G.M.J. Mohren, P. Copini en J. den Ouden

Gewone Esdoorn door J.J. Jansen, A. Oosterbaan, G.M.J. Mohren en J. den Ouden

Dit rapport is gratis te downloaden op: www.wageningenacademic.com/opbrengsttabellen. 


\section{Voorwoord}

In Nederland is met behulp van permanente proef- en steekproefperken groei- en productieonderzoek gedaan in gelijkjarige monoculturen van boomsoorten. De waarnemingen liggen ongeveer tussen 1920 en 2010, maar het merendeel van die waarnemingen ligt tussen 1950 en 2000. Voortrekkers hierbij waren De Exotencommissie ${ }^{1}$, en vanaf 1950 Becking op de Landbouwhogeschool, Van Soest op de Dorschkamp en Grandjean en Stoffels bij het Staatsbosbeheer. Bartelink et al. (2001) geven een uitgebreid overzicht van de context en publicaties van het groei- en productieonderzoek aan boomsoorten in Nederland. Samen met Anne Oosterbaan heb ik deze data voor zover te achterhalen in een set databases opgenomen op de DANS-site van de KNAW, welke te raadplegen is via https://doi.org/10.17026/dans-zan-sjhm. Daarin zijn ook databases met gegevens van gemengd bos en van ongelijkjarig bos opgenomen.

Hans Jansen,

Wageningen, 2018

1 "Commissie tot onderzoek van exotische coniferen" opgericht door de Nederlandsche Heidemaatschappij in 1899.

2 Bartelink, H.H., A.F.M. Olsthoorn, A. Oosterbaan \& S.M.J. Wijdeven, 2001. Overzicht van een eeuw onderzoek naar groei en opstandsontwikkeling in relatie tot groeiplaats en beheer. Alterra, Research Instituut voor de Groene Ruimte, Wageningen, Alterra-rapport 256.
De eerste set opbrengsttabellen ${ }^{3}$ voor gebruik in Nederland is in 1959 verschenen als Bijlage 10 (IV) bij het "Groene boekje". Het betrof 11 boomsoorten, waarvan 4 gebaseerd op Nederlandse gegevens. Jansen et al. ${ }^{4}$ publiceerden in 1996 een opbrengsttabellenboek met 12 boomsoorten, waarvan er 9 gebaseerd waren op Nederlandse gegevens. In het huidige boek komen de opbrengsttabellen van 15 boomsoorten aan de orde, alle gebaseerd op Nederlandse gegevens.

Anders dan bij Jansen et al. (1996) wordt er naast de tabellen geen uitvoerige uitleg over de modellen gegeven, Hiervoor wordt verwezen naar de afzonderlijke rapporten per soort (een verwijzing is per soort opgenomen). Voor de toelichting op het gebruik van opbrengsttabellen, definities van de gebruikte begrippen is het boek van Jansen et al. (1996) nog steeds aan te bevelen.

In de rapporten van de afzonderlijke tabellen per soort zijn vaak meerdere varianten (dunning of plantverband) opgenomen; in dit boek is daar een selectie uit opgenomen. Bij boomsoorten met voldoende data een matige en een sterke dunning, anders een matige dunning of een sterke dunning. De meeste tabellen komen rechtstreeks uit de rapporten, in sommige gevallen is er met de gepubliceerde modellen een andere variant berekend. Bij populier is gekozen voor de plantverbanden $4 \times 4,5 \times 5$ en $5 \times 6 \mathrm{~m}$ en voor lijnbeplantingen met een plantverband van $3,2 \mathrm{~m}$.

3 Becking, J.H. en P.G. de Vries, 1959. Richtlijnen voor de bedrijfsregeling van bosbezit in Nederland: samengesteld door de commissie bosbedrijfsregeling van de Nederlandsche Boschbouwvereeniging, ingesteld op 7 januari 1954 (http://edepot.wur.nl/454998)

4 Jansen, J.J., J. Sevenster \& P.G. Faber (redactie), 1996. Opbrengsttabellen voor belangrijke boomsoorten in Nederland. IBN rapport 96/Hinkeloord reports No.17, 202 pag. (http://library.wur.nl/WebQuery/groenekennis/922890) 


\section{Toelichting/Explanation}

Voor alle boomsoorten exclusief (tril)populier.

For all tree species excluding poplar and aspen.

\begin{tabular}{|c|c|c|c|}
\hline symbool & eenheid/unit & betekenis & meaning \\
\hline Boniteit & & relatieve indeling in groeiklassen & site class \\
\hline$h_{\mathrm{xx}}$ & $\mathrm{m}$ & site index (opperhoogte op xx jr) & site index (top height at $x x y r$ ) \\
\hline$t$ & j & leeftijd vanaf kieming & age since germination \\
\hline$h_{\text {top }}$ & $\mathrm{m}$ & opperhoogte & top height \\
\hline$h_{\text {dom }}$ & $\mathrm{m}$ & dominante hoogte & dominant height \\
\hline$d_{d o m}$ & $\mathrm{~cm}$ & $\begin{array}{l}\text { gemiddelde diameter van } \\
\text { dominante hoogte boom }\end{array}$ & mean diameter of dominant tree \\
\hline$S \%$ & & S\% (dunningsindex van Hart) & Hart-Becking spacing index \\
\hline$N$ & $h^{-1}$ & stamtal per ha & density (number of trees) \\
\hline G & $m^{2} h a^{-1}$ & grondvlak per ha & basal area \\
\hline$d_{g}$ & $\mathrm{~cm}$ & $\begin{array}{l}\text { diameter }(1,30 \mathrm{~m}) \text { van de } \\
\text { grondvlakmiddenboom }\end{array}$ & $\begin{array}{l}\text { diameter at breast height of mean } \\
\text { basal area tree }\end{array}$ \\
\hline$h_{g}$ & $\mathrm{~m}$ & $\begin{array}{l}\text { hoogte van de } \\
\text { grondvlakmiddenboom }\end{array}$ & height of mean basal area tree \\
\hline$v$ & $m^{3} h a^{-1}$ & spilvolume met schors & stem volume over bark \\
\hline$I c_{G}$ & $m^{2} h a^{-1} j^{-1}$ & $\begin{array}{l}\text { lopende grondvlakbijgroei op } \\
\text { leeftijd } t \text { jaar }\end{array}$ & current basal area increment at age $t$ \\
\hline $1 c_{v}$ & $m^{3} h a^{-1} j^{-1}$ & $\begin{array}{l}\text { lopende volumebijgroei op leeftijd } \\
t \text { jaar }\end{array}$ & current volume increment at age $t$ \\
\hline $\operatorname{Im} m_{G}$ & $m^{2} h a^{-1} j^{-1}$ & $\begin{array}{l}\text { gemiddelde grondvlakbijgroei tot } \\
\text { op leeftijd } t \text { jaar }\end{array}$ & mean basal area increment until age $t$ \\
\hline $\operatorname{Im} m_{V}$ & $m^{3} h a^{-1} j^{-1}$ & $\begin{array}{l}\text { gemiddelde volumebijgroei tot op } \\
\text { leeftijd } t \text { jaar }\end{array}$ & mean volume increment until age $t$ \\
\hline
\end{tabular}

Voor (tril)populier in bosverband / For poplar and aspen in forest.

\begin{tabular}{|c|c|c|c|}
\hline symbool & eenheid/unit & betekenis & meaning \\
\hline Boniteit & & relatieve indeling in groeiklassen & site class \\
\hline$h_{25}$ & $\mathrm{~m}$ & $\begin{array}{l}\text { site index (gemiddelde hoogte op } \\
25 \text { j) }\end{array}$ & site index (mean height at $25 \mathrm{yr}$ ) \\
\hline$t$ & j & kiem- of stekleeftijd & age since germination or sprouting \\
\hline$h_{m}$ & $\mathrm{~m}$ & gemiddelde hoogte & mean height \\
\hline$S \%$ & & S\% (dunningsindex van Hart) & Hart-Becking spacing index \\
\hline N & $h a^{-1}$ & stamtal per ha & density (number of trees) \\
\hline G & $m^{2} h a^{-1}$ & grondvlak per ha & basal area \\
\hline$d_{g}$ & $\mathrm{~cm}$ & $\begin{array}{l}\text { diameter }(1,30 \mathrm{~m}) \text { van de } \\
\text { grondvlakmiddenboom }\end{array}$ & $\begin{array}{l}\text { diameter at breast height of mean } \\
\text { basal area tree }\end{array}$ \\
\hline v & $m^{3} \mathrm{ha}^{-1}$ & spilvolume met schors & stem volume over bark \\
\hline $1 c_{G}$ & $m^{2} h a^{-1-1}$ & $\begin{array}{l}\text { lopende grondvlakbijgroei op } \\
\text { leeftijd } t \text { jaar }\end{array}$ & current basal area increment at age $t$ \\
\hline$c_{v}$ & $m^{3} h a^{-1} j^{-1}$ & $\begin{array}{l}\text { lopende volumebijgroei op leeftijd } \\
t \text { jaar }\end{array}$ & current volume increment at age $t$ \\
\hline$I m_{G}$ & $m^{2} h a^{-1} j^{-1}$ & $\begin{array}{l}\text { gemiddelde grondvlakbijgroei tot } \\
\text { op leeftijd } t \text { jaar }\end{array}$ & mean basal area increment until age $t$ \\
\hline $\operatorname{Im} m_{V}$ & $m^{3} h a^{-1-1}$ & $\begin{array}{l}\text { gemiddelde volumebijgroei tot op } \\
\text { leeftijd } t \text { jaar }\end{array}$ & mean volume increment until age $t$ \\
\hline
\end{tabular}

Voor populier in lijnbeplanting / For poplar line plantation.

\begin{tabular}{|c|c|c|c|}
\hline symbool & eenheid & betekenis & meaning \\
\hline Boniteit & & relatieve indeling in groeiklassen & site class \\
\hline$h_{25}$ & $\mathrm{~m}$ & Site index gemiddelde hoogte op $25 \mathrm{jr}$ ) & site index (top height at $25 \mathrm{yr}$ ) \\
\hline$t$ & j & leeftijd vanaf kieming & age since germination \\
\hline$h_{m}$ & $\mathrm{~m}$ & gemiddelde hoogte & mean height \\
\hline $5 \%$ & & S\% (dunningindex van Hart) & Hart-Becking spacing index \\
\hline N & $\mathrm{km}^{-1}$ & stamtal & density (number of trees) \\
\hline G & $\mathrm{m}^{2} \mathrm{~km}^{-1}$ & grondvla & basal area \\
\hline$d_{g}$ & $\mathrm{~cm}$ & $\begin{array}{l}\text { diameter }(1,30 \mathrm{~m}) \text { van de } \\
\text { grondvlakmiddenboom }\end{array}$ & $\begin{array}{l}\text { diameter at breast height of mean } \\
\text { basal area tree }\end{array}$ \\
\hline$d_{\text {or }}$ & $\mathrm{cm}$ & diameter loodrecht op rij & diameter on row \\
\hline$d_{i r}$ & $\mathrm{~cm}$ & diameter in de rij & diameter in row \\
\hline$v$ & $\mathrm{~m}^{3} \mathrm{~km}^{-1}$ & spilvolume met schors & stem volume over bark \\
\hline $1 c_{G}$ & $\mathrm{~m}^{2} \mathrm{~km}^{-1} \mathrm{j}^{-1}$ & $\begin{array}{l}\text { lopende grondvlakbijgroei op leeftijd } t \\
\text { jaar }\end{array}$ & current basal area increment at age $t$ \\
\hline$I c_{V}$ & $\mathrm{~m}^{3} \mathrm{~km}^{-1-\mathrm{j}^{-1}}$ & lopende volumebijgroei op leeftijd $t$ jaar & current volume increment at age $t$ \\
\hline$I m_{G}$ & $\mathrm{~m}^{2} \mathrm{~km}^{-1} \mathrm{j}^{-1}$ & $\begin{array}{l}\text { gemiddelde grondvlakbijgroei tot op } \\
\text { leeftijd } t \text { jaar }\end{array}$ & mean basal area increment until age $t$ \\
\hline $\operatorname{Im} v$ & $\mathrm{~m}^{3} \mathrm{~km}^{-1-1}$ & $\begin{array}{l}\text { gemiddelde volumebijgroei tot op leeftijd } \\
t \text { jaar }\end{array}$ & mean volume increment until age $t$ \\
\hline
\end{tabular}




\section{Grove den (Pinus sylvestris)}

Scots pine

Jansen, J.J., G.M.J. Mohren, A. Oosterbaan, L. Goudzwaard, en J. den

Ouden

Bron: Jansen, J.J., G.M.J. Mohren, A. Oosterbaan, L. Goudzwaard, en J. den Ouden, 2018. Groei en productie van grove den in Nederland. FEM Groei en Productie Rapport 2018 - 3, 87 blz.

\section{Dit rapport is gratis te downloaden}

op: https://doi.org/10.18174/444090

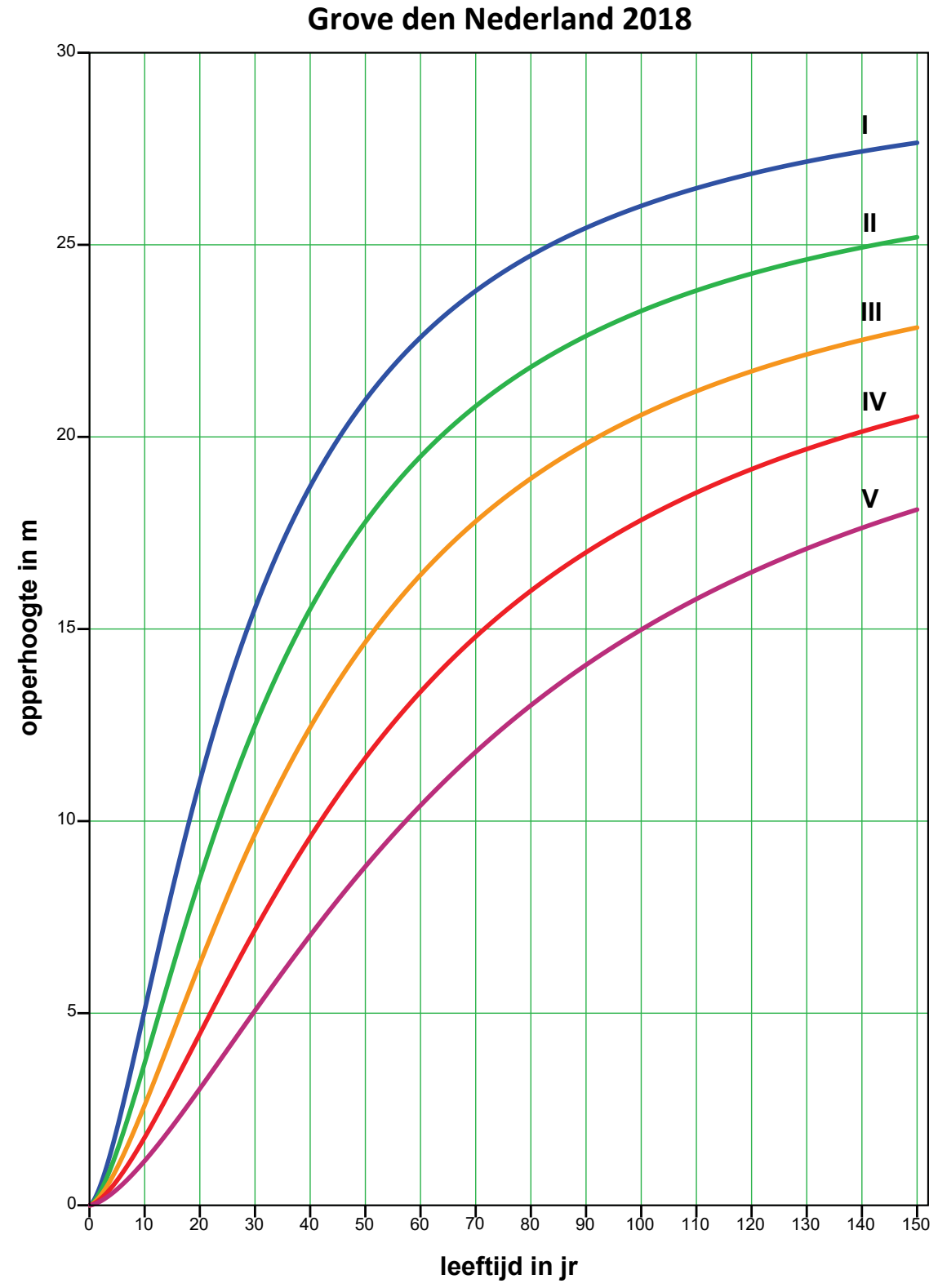




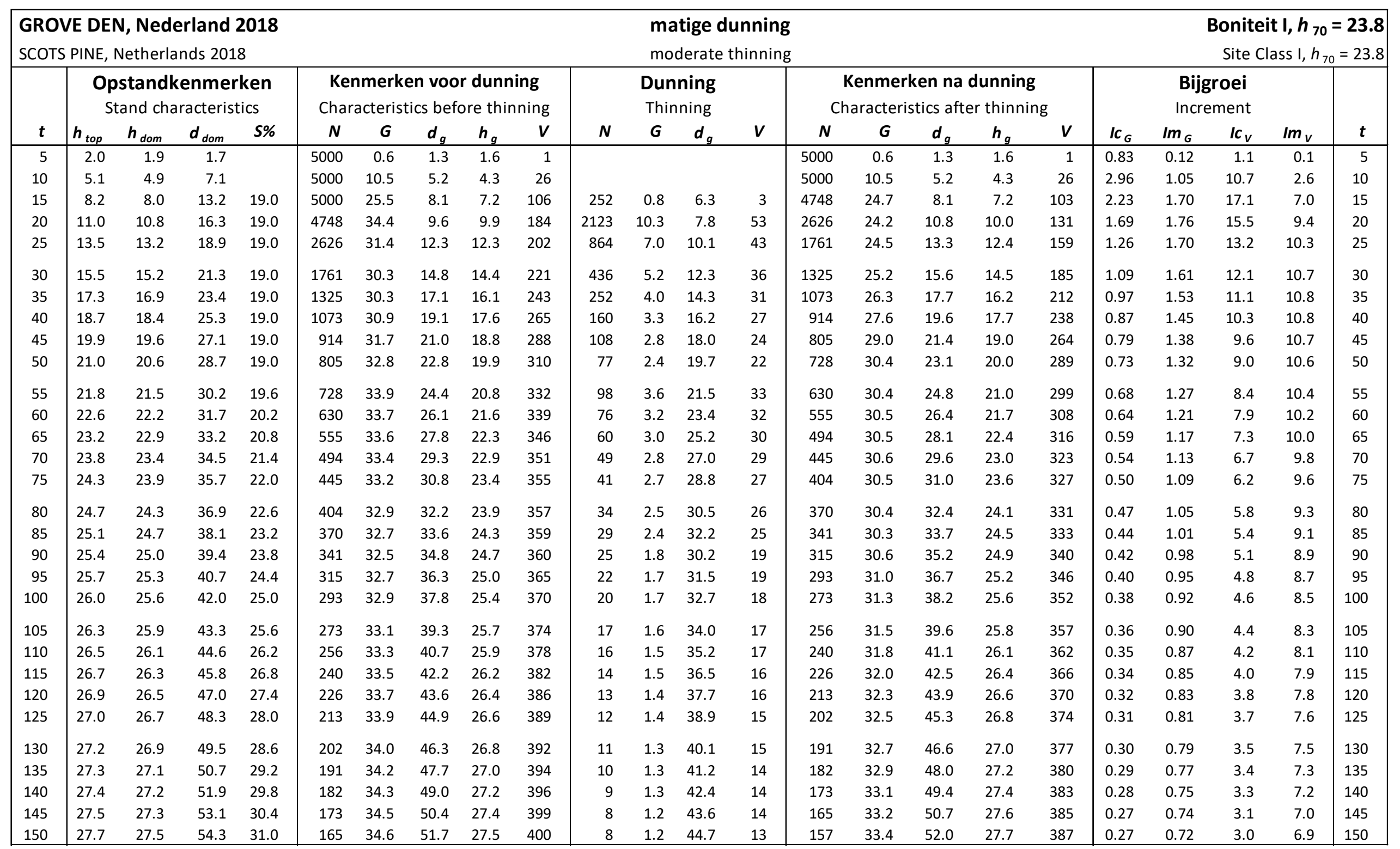




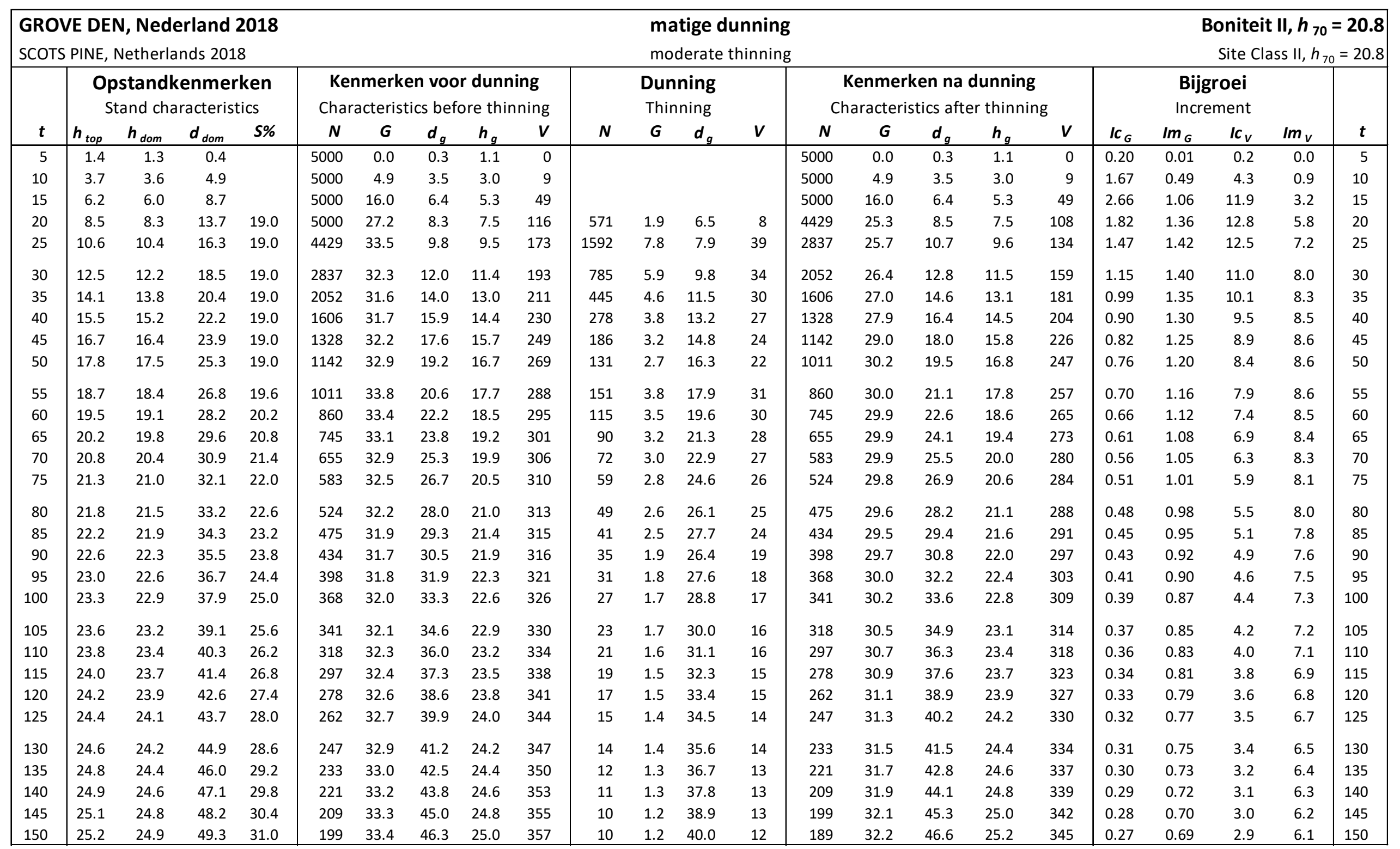




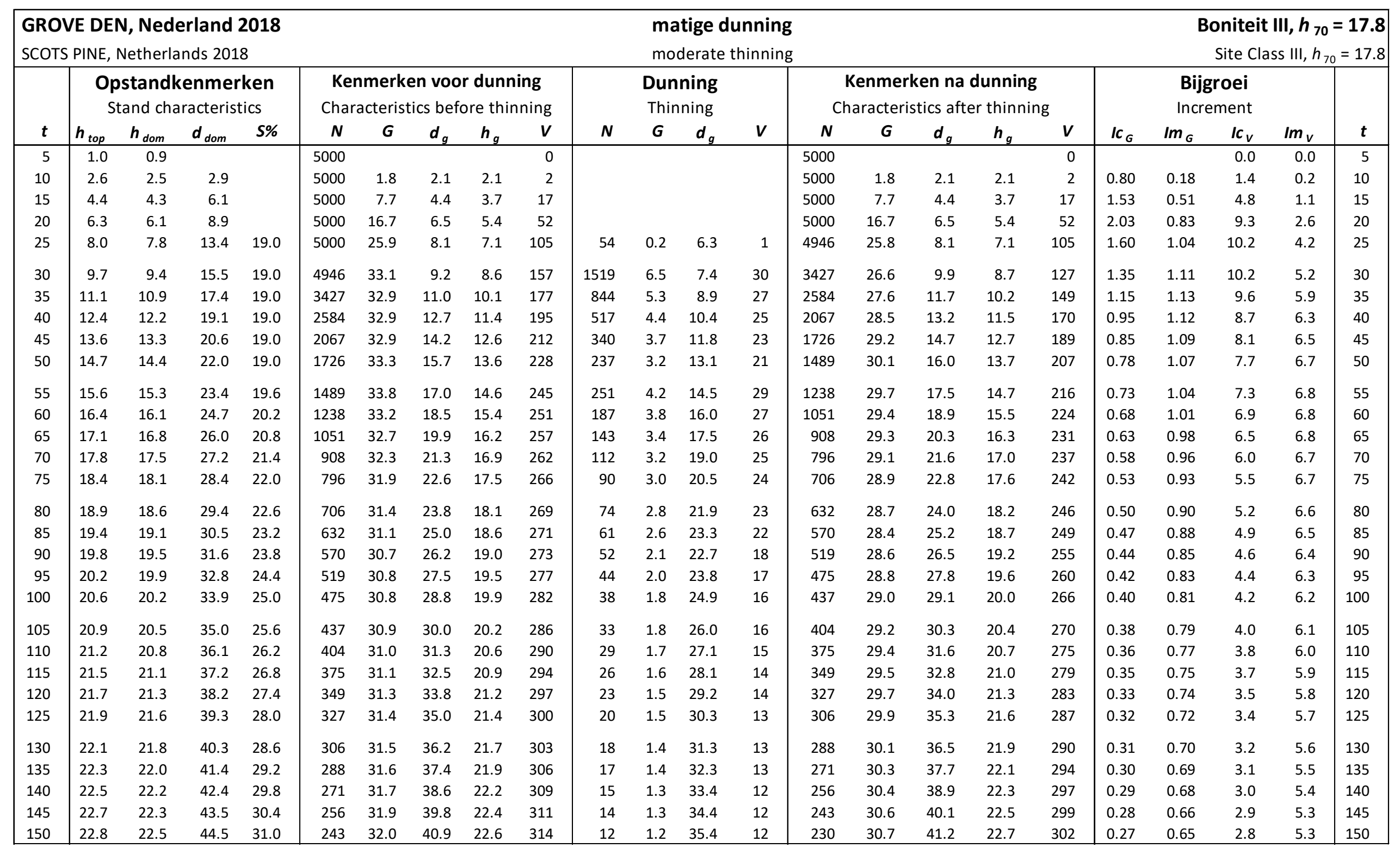




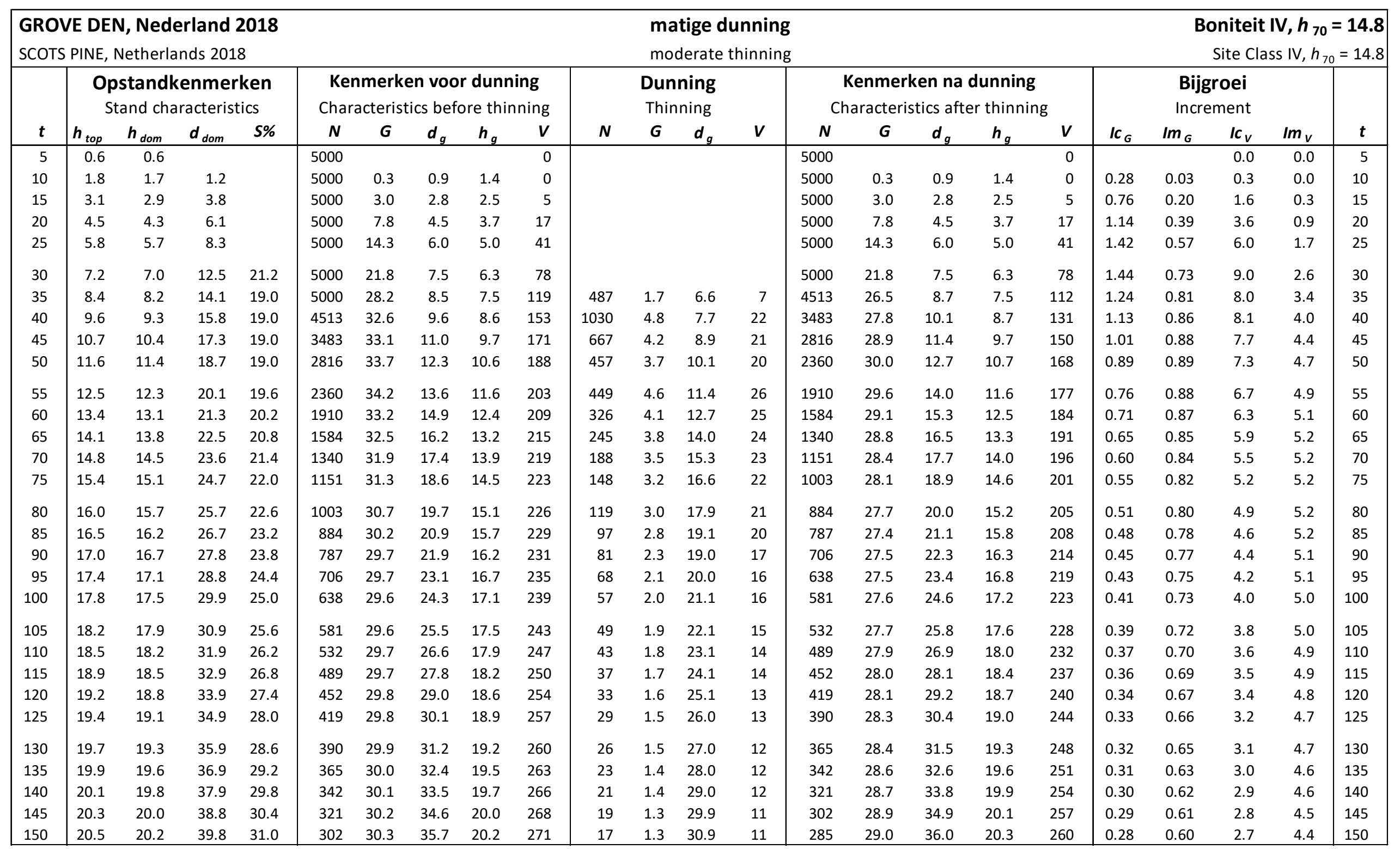




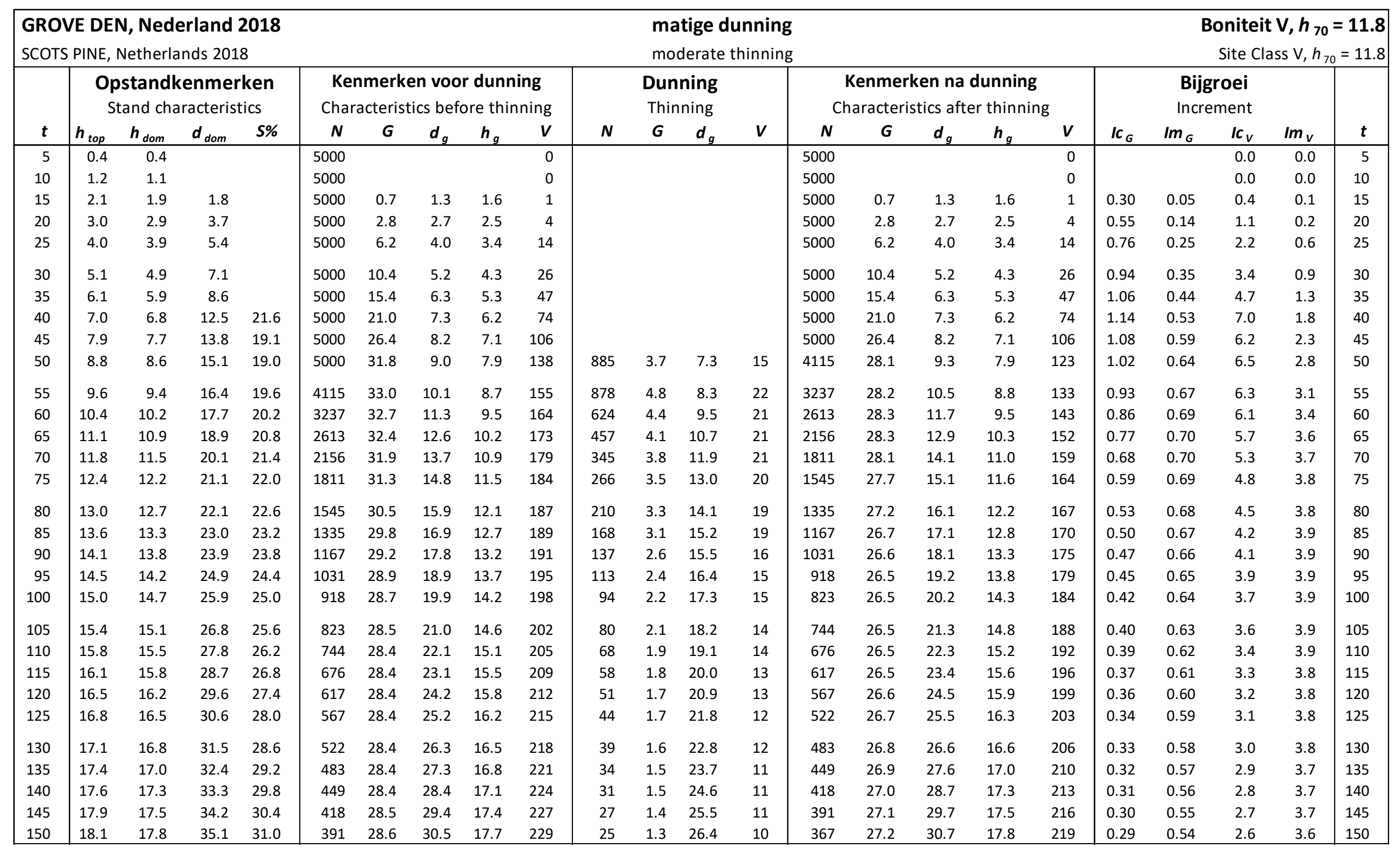




\begin{tabular}{|c|c|c|c|c|c|c|c|c|c|c|c|c|c|c|c|c|c|c|c|c|c|c|c|}
\hline \multirow{2}{*}{\multicolumn{5}{|c|}{$\begin{array}{l}\text { GROVE DEN, Nederland } 2018 \\
\text { SCOTS PINE, Netherlands } 2018\end{array}$}} & \multicolumn{15}{|c|}{ sterke dunning } & \multicolumn{4}{|c|}{ Boniteit I, $h_{70}=23.8$} \\
\hline & & & & & & & & & & & & eavy $t$ & ning & & & & & & & & Site & ass I, $h$ & $=23.8$ \\
\hline \multirow[b]{2}{*}{$t$} & \multicolumn{4}{|c|}{$\begin{array}{l}\text { Opstandkenmerken } \\
\text { Stand characteristics }\end{array}$} & \multicolumn{5}{|c|}{$\begin{array}{l}\text { Kenmerken voor dunning } \\
\text { Characteristics before thinning }\end{array}$} & \multicolumn{4}{|c|}{$\begin{array}{l}\text { Dunning } \\
\text { Thinning }\end{array}$} & \multicolumn{5}{|c|}{$\begin{array}{l}\text { Kenmerken na dunning } \\
\text { Characteristics after thinning }\end{array}$} & \multicolumn{4}{|c|}{$\begin{array}{l}\text { Bijgroei } \\
\text { Increment }\end{array}$} & \multirow[b]{2}{*}{$t$} \\
\hline & $h_{\text {top }}$ & $\boldsymbol{h}_{\text {dom }}$ & $d_{d o m}$ & $S \%$ & $N$ & $\boldsymbol{G}$ & $d_{g}$ & $\boldsymbol{h}_{g}$ & $\boldsymbol{v}$ & $N$ & $\boldsymbol{G}$ & $d_{g}$ & $\boldsymbol{V}$ & $N$ & $\boldsymbol{G}$ & $d_{g}$ & $\boldsymbol{h}_{g}$ & $\boldsymbol{V}$ & $I c_{G}$ & $I m_{G}$ & $I c_{v}$ & $\operatorname{Im} m_{V}$ & \\
\hline 5 & 2.0 & 1.9 & 1.7 & & 5000 & 0.6 & 1.3 & 1.6 & 1 & & & & & 5000 & 0.6 & 1.3 & 1.6 & 1 & 0.83 & 0.12 & 1.1 & 0.1 & 5 \\
\hline 10 & 5.1 & 4.9 & 7.1 & & 5000 & 10.5 & 5.2 & 4.3 & 26 & & & & & 5000 & 10.5 & 5.2 & 4.3 & 26 & 2.96 & 1.05 & 10.7 & 2.6 & 10 \\
\hline 15 & 8.2 & 8.0 & 13.4 & 22.0 & 5000 & 25.1 & 8.0 & 7.2 & 104 & 1458 & 4.8 & 6.5 & 19 & 3542 & 20.2 & 8.5 & 7.2 & 85 & 2.02 & 1.67 & 15.6 & 6.9 & 15 \\
\hline 20 & $\mid$\begin{tabular}{|l|}
11.0 \\
\end{tabular} & 10.8 & 16.6 & 22.0 & 3542 & 29.0 & 10.2 & 9.9 & 156 & 1583 & 9.0 & 8.5 & 47 & 1958 & 20.0 & 11.4 & 10.0 & 110 & 1.53 & 1.69 & 13.7 & 8.8 & 20 \\
\hline 25 & 13.5 & 13.2 & 19.5 & 22.0 & 1958 & 26.6 & 13.2 & 12.3 & 172 & 645 & 6.1 & 11.0 & 38 & 1314 & 20.5 & 14.1 & 12.4 & 134 & 1.14 & 1.62 & 11.7 & 9.5 & 25 \\
\hline 30 & 15.5 & 15.2 & 22.0 & 22.0 & 1314 & 25.8 & 15.8 & 14.4 & 189 & 325 & 4.6 & 13.3 & 32 & 988 & 21.2 & 16.5 & 14.5 & 157 & 0.99 & 1.52 & 10.8 & 9.8 & 30 \\
\hline 35 & \begin{tabular}{|l}
17.3 \\
\end{tabular} & 16.9 & 24.3 & 22.0 & 988 & 25.9 & 18.3 & 16.1 & 209 & 188 & 3.6 & 15.6 & 28 & 800 & 22.3 & 18.8 & 16.2 & 181 & 0.87 & 1.44 & 10.0 & 9.9 & 35 \\
\hline 40 & \begin{tabular}{|l}
18.7 \\
\end{tabular} & 18.4 & 26.3 & 22.0 & 800 & 26.4 & 20.5 & 17.6 & 229 & 119 & 2.9 & 17.7 & 24 & 681 & 23.5 & 21.0 & 17.7 & 204 & 0.79 & 1.36 & 9.3 & 9.8 & 40 \\
\hline 45 & 19.9 & 19.6 & 28.2 & 22.0 & 681 & 27.3 & 22.6 & 18.8 & 249 & 81 & 2.5 & 19.7 & 22 & 601 & 24.8 & 22.9 & 19.0 & 227 & 0.72 & 1.30 & 8.6 & 9.7 & 45 \\
\hline 50 & 21.0 & 20.6 & 30.0 & 22.0 & 601 & 28.3 & 24.5 & 19.9 & 269 & 58 & 2.1 & 21.6 & 19 & 543 & 26.2 & 24.8 & 20.0 & 250 & 0.66 & 1.23 & 8.1 & 9.6 & 50 \\
\hline 55 & 21.8 & 21.5 & 31.6 & 22.6 & 543 & 29.4 & 26.2 & 20.8 & 289 & 69 & 3.0 & 23.5 & 28 & 474 & 26.4 & 26.6 & 21.0 & 261 & 0.61 & 1.18 & 7.5 & 9.4 & 55 \\
\hline 60 & 22.6 & 22.2 & 33.2 & 23.2 & 474 & 29.3 & 28.0 & 21.6 & 297 & 54 & 2.7 & 25.5 & 27 & 420 & 26.5 & 28.4 & 21.7 & 270 & 0.56 & 1.13 & 6.9 & 9.2 & 60 \\
\hline 65 & 23.2 & 22.9 & 34.7 & 23.8 & 420 & 29.3 & 29.8 & 22.3 & 303 & 43 & 2.5 & 27.5 & 25 & 378 & 26.7 & 30.0 & 22.4 & 278 & 0.52 & 1.09 & 6.4 & 9.1 & 65 \\
\hline 70 & 23.8 & 23.4 & 36.1 & 24.4 & 378 & 29.2 & 31.4 & 22.9 & 309 & 35 & 2.4 & 29.4 & 24 & 342 & 26.9 & 31.6 & 23.0 & 285 & 0.49 & 1.04 & 6.0 & 8.8 & 70 \\
\hline 75 & 24.3 & 23.9 & 37.4 & 25.0 & 342 & 29.2 & 33.0 & 23.4 & 314 & 29 & 2.2 & 31.2 & 23 & 313 & 27.0 & 33.1 & 23.6 & 291 & 0.46 & 1.01 & 5.6 & 8.6 & 75 \\
\hline 80 & \begin{tabular}{|l|}
24.7 \\
\end{tabular} & 24.3 & 38.7 & 25.6 & 313 & 29.2 & 34.5 & 23.9 & 318 & 25 & 2.1 & 33.1 & 22 & 288 & 27.1 & 34.6 & 24.1 & 296 & 0.43 & 0.97 & 5.3 & 8.4 & 80 \\
\hline 85 & 25.1 & 24.7 & 39.9 & 26.2 & 288 & 29.2 & 35.9 & 24.3 & 321 & 21 & 2.0 & 34.9 & 22 & 267 & 27.1 & 36.0 & 24.5 & 300 & 0.41 & 0.94 & 5.0 & 8.3 & 85 \\
\hline 90 & 25.4 & 25.0 & 41.4 & 26.8 & 267 & 29.2 & 37.3 & 24.7 & 324 & 19 & 1.5 & 32.3 & 16 & 248 & 27.6 & 37.6 & 24.9 & 308 & 0.39 & 0.91 & 4.7 & 8.1 & 90 \\
\hline 95 & 25.7 & 25.4 & 42.8 & 27.4 & 248 & 29.5 & 38.9 & 25.0 & 331 & 16 & 1.4 & 33.7 & 16 & 232 & 28.1 & 39.3 & 25.2 & 316 & 0.37 & 0.88 & 4.5 & 7.9 & 95 \\
\hline 100 & 26.0 & 25.7 & 44.2 & 28.0 & 232 & 29.9 & 40.5 & 25.4 & 338 & 14 & 1.4 & 35.0 & 15 & 218 & 28.5 & 40.8 & 25.6 & 322 & 0.36 & 0.86 & 4.3 & 7.7 & 100 \\
\hline 105 & 26.3 & 25.9 & 45.6 & 28.6 & 218 & 30.3 & 42.1 & 25.7 & 343 & 13 & 1.3 & 36.4 & 15 & 205 & 28.9 & 42.4 & 25.8 & 329 & 0.34 & 0.83 & 4.1 & 7.5 & 105 \\
\hline 110 & 26.5 & 26.2 & 47.0 & 29.2 & 205 & 30.6 & 43.6 & 25.9 & 349 & 12 & 1.3 & 37.7 & 14 & 193 & 29.3 & 43.9 & 26.1 & 335 & 0.33 & 0.81 & 3.9 & 7.4 & 110 \\
\hline 115 & 26.7 & 26.4 & 48.3 & 29.8 & 193 & 30.9 & 45.1 & 26.2 & 354 & 10 & 1.3 & 39.0 & 14 & 183 & 29.7 & 45.5 & 26.4 & 340 & 0.32 & 0.79 & 3.8 & 7.2 & 115 \\
\hline 120 & 26.9 & 26.6 & 49.7 & 30.4 & 183 & 31.2 & 46.6 & 26.4 & 359 & 9 & 1.2 & 40.3 & 13 & 173 & 30.0 & 47.0 & 26.6 & 345 & 0.31 & 0.77 & 3.6 & 7.1 & 120 \\
\hline 125 & 27.0 & 26.8 & 51.0 & 31.0 & 173 & 31.5 & 48.1 & 26.6 & 363 & 9 & 1.2 & 41.6 & 13 & 165 & 30.4 & 48.4 & 26.8 & 350 & 0.30 & 0.75 & 3.5 & 6.9 & 125 \\
\hline 130 & 27.2 & 27.0 & 52.3 & 31.6 & 165 & 31.8 & 49.6 & 26.8 & 367 & 8 & 1.1 & 42.9 & 13 & 157 & 30.7 & 49.9 & 27.0 & 354 & 0.29 & 0.73 & 3.3 & 6.8 & 130 \\
\hline 135 & 27.3 & 27.2 & 53.6 & 32.2 & 157 & 32.1 & 51.0 & 27.0 & 371 & 7 & 1.1 & 44.1 & 12 & 149 & 30.9 & 51.3 & 27.2 & 358 & 0.28 & 0.71 & 3.2 & 6.7 & 135 \\
\hline 140 & 27.4 & 27.3 & 54.9 & 32.8 & 149 & 32.3 & 52.5 & 27.2 & 374 & 7 & 1.1 & 45.4 & 12 & 143 & 31.2 & 52.8 & 27.4 & 362 & 0.27 & 0.70 & 3.1 & 6.5 & 140 \\
\hline 145 & 27.5 & 27.4 & 56.2 & 33.4 & 143 & 32.5 & 53.9 & 27.4 & 377 & 6 & 1.1 & 46.6 & 12 & 136 & 31.5 & 54.2 & 27.6 & 365 & 0.26 & 0.68 & 3.0 & 6.4 & 145 \\
\hline 150 & 27.7 & 27.6 & 57.5 & 34.0 & 136 & 32.8 & 55.3 & 27.5 & 380 & 6 & 1.0 & 47.8 & 12 & 131 & 31.7 & 55.6 & 27.7 & 368 & 0.25 & 0.67 & 2.9 & 6.3 & 150 \\
\hline
\end{tabular}




\begin{tabular}{|c|c|c|c|c|c|c|c|c|c|c|c|c|c|c|c|c|c|c|c|c|c|c|c|}
\hline \multirow{2}{*}{\multicolumn{5}{|c|}{$\begin{array}{l}\text { GROVE DEN, Nederland } 2018 \\
\text { SCOTS PINE, Netherlands } 2018\end{array}$}} & \multicolumn{15}{|c|}{ sterke dunning } & \multicolumn{4}{|c|}{ Boniteit II, $h_{70}=20.8$} \\
\hline & & & & & & & & & & & & eavy $t$ & ining & & & & & & & & Site $C$ & ss II, $h$ & $=20.8$ \\
\hline \multirow[b]{2}{*}{$t$} & \multicolumn{4}{|c|}{$\begin{array}{l}\text { Opstandkenmerken } \\
\text { Stand characteristics }\end{array}$} & \multicolumn{5}{|c|}{$\begin{array}{l}\text { Kenmerken voor dunning } \\
\text { Characteristics before thinning }\end{array}$} & \multicolumn{4}{|c|}{$\begin{array}{l}\text { Dunning } \\
\text { Thinning }\end{array}$} & \multicolumn{5}{|c|}{$\begin{array}{l}\text { Kenmerken na dunning } \\
\text { Characteristics after thinning }\end{array}$} & \multicolumn{4}{|c|}{$\begin{array}{l}\text { Bijgroei } \\
\text { Increment }\end{array}$} & \multirow[b]{2}{*}{$t$} \\
\hline & $h_{\text {top }}$ & $\boldsymbol{h}_{\text {dom }}$ & $d_{d o m}$ & $S \%$ & $N$ & $\boldsymbol{G}$ & $d_{g}$ & $\boldsymbol{h}_{g}$ & $\boldsymbol{v}$ & $N$ & $\boldsymbol{G}$ & $d_{g}$ & $\boldsymbol{V}$ & $N$ & $\boldsymbol{G}$ & $d_{g}$ & $\boldsymbol{h}_{g}$ & $\boldsymbol{V}$ & $I c_{G}$ & $I m_{G}$ & $I c_{v}$ & $\operatorname{Im} m_{V}$ & \\
\hline 5 & 1.4 & 1.3 & 0.4 & & 5000 & 0.0 & 0.3 & 1.1 & 0 & & & & & 5000 & 0.0 & 0.3 & 1.1 & 0 & 0.20 & 0.01 & 0.2 & 0.0 & 5 \\
\hline 10 & 3.7 & 3.6 & 4.9 & & 5000 & 4.9 & 3.5 & 3.0 & 9 & & & & & 5000 & 4.9 & 3.5 & 3.0 & 9 & 1.67 & 0.49 & 4.3 & 0.9 & 10 \\
\hline 15 & 6.2 & 6.0 & 8.7 & & 5000 & 16.0 & 6.4 & 5.3 & 49 & & & & & 5000 & 16.0 & 6.4 & 5.3 & 49 & 2.66 & 1.06 & 11.9 & 3.2 & 15 \\
\hline 20 & 8.5 & 8.3 & 13.9 & 22.0 & 5000 & 26.6 & 8.2 & 7.5 & 114 & 1697 & 5.9 & 6.7 & 24 & 3303 & 20.7 & 8.9 & 7.5 & 89 & 1.65 & 1.33 & 11.6 & 5.7 & 20 \\
\hline 25 & 10.6 & 10.4 & 16.6 & 22.0 & 3303 & 28.1 & 10.4 & 9.5 & 146 & 1187 & 6.8 & 8.6 & 34 & 2116 & 21.3 & 11.3 & 9.6 & 112 & 1.33 & 1.36 & 11.0 & 6.8 & 25 \\
\hline 30 & 12.5 & 12.2 & 19.0 & 22.0 & 2116 & 27.2 & 12.8 & 11.4 & 164 & 586 & 5.2 & 10.6 & 30 & 1530 & 22.0 & 13.5 & 11.5 & 134 & 1.04 & 1.33 & 9.8 & 7.4 & 30 \\
\hline 35 & $\mid 14.1$ & 13.8 & 21.1 & 22.0 & 1530 & 26.8 & 14.9 & 13.0 & 180 & 332 & 4.1 & 12.5 & 26 & 1198 & 22.7 & 15.5 & 13.1 & 154 & 0.90 & 1.28 & 9.0 & 7.7 & 35 \\
\hline 40 & 15.5 & 15.2 & 23.0 & 22.0 & 1198 & 27.0 & 16.9 & 14.4 & 197 & 207 & 3.4 & 14.4 & 24 & 991 & 23.6 & 17.4 & 14.5 & 174 & 0.81 & 1.23 & 8.5 & 7.8 & 40 \\
\hline 45 & \begin{tabular}{|l|}
16.7 \\
\end{tabular} & 16.4 & 24.7 & 22.0 & 991 & 27.5 & 18.8 & 15.7 & 214 & 139 & 2.8 & 16.1 & 21 & 852 & 24.7 & 19.2 & 15.8 & 193 & 0.74 & 1.18 & 8.0 & 7.9 & 45 \\
\hline 50 & 17.8 & 17.5 & 26.4 & 22.0 & 852 & 28.2 & 20.5 & 16.7 & 232 & 98 & 2.4 & 17.8 & 19 & 754 & 25.8 & 20.9 & 16.8 & 213 & 0.68 & 1.13 & 7.5 & 7.8 & 50 \\
\hline 55 & 18.7 & 18.4 & 27.9 & 22.6 & 754 & 29.1 & 22.2 & 17.7 & 249 & 108 & 3.2 & 19.6 & 27 & 647 & 25.8 & 22.6 & 17.8 & 222 & 0.63 & 1.09 & 7.0 & 7.8 & 55 \\
\hline 60 & 19.5 & 19.1 & 29.4 & 23.2 & 647 & 28.9 & 23.8 & 18.5 & 256 & 82 & 2.9 & 21.3 & 25 & 565 & 25.9 & 24.2 & 18.6 & 231 & 0.58 & 1.05 & 6.5 & 7.7 & 60 \\
\hline 65 & 20.2 & 19.8 & 30.8 & 23.8 & 565 & 28.7 & 25.4 & 19.2 & 263 & 64 & 2.7 & 23.1 & 24 & 500 & 26.0 & 25.7 & 19.4 & 239 & 0.54 & 1.01 & 6.1 & 7.6 & 65 \\
\hline 70 & 20.8 & 20.4 & 32.1 & 24.4 & 500 & 28.6 & 27.0 & 19.9 & 268 & 52 & 2.5 & 24.8 & 23 & 448 & 26.1 & 27.2 & 20.0 & 245 & 0.50 & 0.97 & 5.7 & 7.5 & 70 \\
\hline 75 & 21.3 & 21.0 & 33.4 & 25.0 & 448 & 28.5 & 28.5 & 20.5 & 273 & 43 & 2.4 & 26.6 & 22 & 406 & 26.1 & 28.6 & 20.6 & 251 & 0.47 & 0.94 & 5.3 & 7.3 & 75 \\
\hline 80 & \begin{tabular}{|l|l} 
& 21.8 \\
\end{tabular} & 21.5 & 34.6 & 25.6 & 406 & 28.4 & 29.9 & 21.0 & 277 & 36 & 2.2 & 28.2 & 21 & 370 & 26.2 & 30.0 & 21.1 & 256 & 0.44 & 0.91 & 5.0 & 7.2 & 80 \\
\hline 85 & 22.2 & 21.9 & 35.8 & 26.2 & 370 & 28.4 & 31.2 & 21.4 & 281 & 30 & 2.1 & 29.9 & 20 & 340 & 26.2 & 31.3 & 21.6 & 260 & 0.42 & 0.88 & 4.8 & 7.1 & 85 \\
\hline 90 & 22.6 & 22.3 & 37.1 & 26.8 & 340 & 28.3 & 32.5 & 21.9 & 284 & 26 & 1.6 & 28.2 & 16 & 314 & 26.7 & 32.9 & 22.0 & 268 & 0.40 & 0.86 & 4.5 & 6.9 & 90 \\
\hline 95 & 23.0 & 22.6 & 38.4 & 27.4 & 314 & 28.6 & 34.1 & 22.3 & 290 & 22 & 1.5 & 29.5 & 15 & 292 & 27.1 & 34.4 & 22.4 & 275 & 0.38 & 0.83 & 4.3 & 6.8 & 95 \\
\hline 100 & 23.3 & 22.9 & 39.8 & 28.0 & 292 & 28.9 & 35.5 & 22.6 & 296 & 20 & 1.5 & 30.8 & 14 & 272 & 27.5 & 35.9 & 22.8 & 282 & 0.36 & 0.81 & 4.1 & 6.7 & 100 \\
\hline 105 & 23.6 & 23.2 & 41.0 & 28.6 & 272 & 29.3 & 37.0 & 22.9 & 302 & 17 & 1.4 & 32.0 & 14 & 254 & 27.9 & 37.3 & 23.1 & 288 & 0.35 & 0.79 & 3.9 & 6.5 & 105 \\
\hline 110 & 23.8 & 23.4 & 42.3 & 29.2 & 254 & 29.6 & 38.5 & 23.2 & 307 & 16 & 1.3 & 33.3 & 13 & 239 & 28.2 & 38.8 & 23.4 & 294 & 0.33 & 0.77 & 3.8 & 6.4 & 110 \\
\hline 115 & 24.0 & 23.7 & 43.6 & 29.8 & 239 & 29.9 & 39.9 & 23.5 & 312 & 14 & 1.3 & 34.5 & 13 & 225 & 28.6 & 40.2 & 23.7 & 299 & 0.32 & 0.75 & 3.6 & 6.3 & 115 \\
\hline 120 & 24.2 & 23.9 & 44.8 & 30.4 & 225 & 30.1 & 41.3 & 23.8 & 316 & 13 & 1.3 & 35.7 & 13 & 213 & 28.9 & 41.6 & 23.9 & 304 & 0.31 & 0.73 & 3.5 & 6.2 & 120 \\
\hline 125 & 24.4 & 24.2 & 46.0 & 31.0 & 213 & 30.4 & 42.7 & 24.0 & 321 & 11 & 1.2 & 36.9 & 12 & 201 & 29.2 & 43.0 & 24.2 & 308 & 0.30 & 0.71 & 3.3 & 6.1 & 125 \\
\hline 130 & 24.6 & 24.4 & 47.2 & 31.6 & 201 & 30.7 & 44.0 & 24.2 & 325 & 10 & 1.2 & 38.1 & 12 & 191 & 29.5 & 44.3 & 24.4 & 313 & 0.29 & 0.70 & 3.2 & 6.0 & 130 \\
\hline 135 & 24.8 & 24.5 & 48.4 & 32.2 & 191 & 30.9 & 45.4 & 24.4 & 328 & 9 & 1.1 & 39.3 & 12 & 181 & 29.8 & 45.7 & 24.6 & 317 & 0.28 & 0.68 & 3.1 & 5.9 & 135 \\
\hline 140 & 24.9 & 24.7 & 49.6 & 32.8 & 181 & 31.1 & 46.8 & 24.6 & 332 & 9 & 1.1 & 40.4 & 11 & 173 & 30.0 & 47.0 & 24.8 & 320 & 0.27 & 0.67 & 3.0 & 5.8 & 140 \\
\hline 145 & 25.1 & 24.9 & 50.8 & 33.4 & 173 & 31.4 & 48.1 & 24.8 & 335 & 8 & 1.1 & 41.6 & 11 & 165 & 30.3 & 48.4 & 25.0 & 324 & 0.26 & 0.65 & 2.9 & 5.7 & 145 \\
\hline 150 & 25.2 & 25.0 & 52.0 & 34.0 & 165 & 31.6 & 49.4 & 25.0 & 338 & 7 & 1.1 & 42.7 & 11 & 157 & 30.5 & 49.7 & 25.2 & 327 & 0.26 & 0.64 & 2.8 & 5.6 & 150 \\
\hline
\end{tabular}




\begin{tabular}{|c|c|c|c|c|c|c|c|c|c|c|c|c|c|c|c|c|c|c|c|c|c|c|c|}
\hline \multirow{2}{*}{\multicolumn{5}{|c|}{$\begin{array}{l}\text { GROVE DEN, Nederland } 2018 \\
\text { SCOTS PINE, Netherlands } 2018\end{array}$}} & \multicolumn{15}{|c|}{ sterke dunning } & \multirow{2}{*}{\multicolumn{4}{|c|}{$\begin{array}{r}\text { Boniteit III, } \boldsymbol{h}_{70}=\mathbf{1 7 . 8} \\
\text { Site Class III, } h_{70}=17.8\end{array}$}} \\
\hline & & & & & & & & & & & & eavy $t$ & ining & & & & & & & & & & \\
\hline \multirow[b]{2}{*}{$t$} & \multicolumn{4}{|c|}{$\begin{array}{l}\text { Opstandkenmerken } \\
\text { Stand characteristics }\end{array}$} & \multicolumn{5}{|c|}{$\begin{array}{l}\text { Kenmerken voor dunning } \\
\text { Characteristics before thinning }\end{array}$} & \multicolumn{4}{|c|}{$\begin{array}{l}\text { Dunning } \\
\text { Thinning }\end{array}$} & \multicolumn{5}{|c|}{$\begin{array}{l}\text { Kenmerken na dunning } \\
\text { Characteristics after thinning }\end{array}$} & \multicolumn{4}{|c|}{$\begin{array}{l}\text { Bijgroei } \\
\text { Increment }\end{array}$} & \multirow[b]{2}{*}{$t$} \\
\hline & $\boldsymbol{h}_{\text {top }}$ & $\boldsymbol{h}_{\text {dom }}$ & $d_{d o m}$ & $S \%$ & $N$ & $\boldsymbol{G}$ & $d_{g}$ & $\boldsymbol{h}_{g}$ & $v$ & $N$ & $\boldsymbol{G}$ & $d_{g}$ & $v$ & $N$ & $\boldsymbol{G}$ & $d_{g}$ & $\boldsymbol{h}_{g}$ & $V$ & $I c_{G}$ & $I m_{G}$ & $I c_{v}$ & $I m_{V}$ & \\
\hline 5 & 1.0 & 0.9 & & & 5000 & & & & 0 & & & & & 5000 & & & & 0 & & & 0.0 & 0.0 & 5 \\
\hline 10 & 2.6 & 2.5 & 2.9 & & 5000 & 1.8 & 2.1 & 2.1 & 2 & & & & & 5000 & 1.8 & 2.1 & 2.1 & 2 & 0.80 & 0.18 & 1.4 & 0.2 & 10 \\
\hline 15 & 4.4 & 4.3 & 6.1 & & 5000 & 7.7 & 4.4 & 3.7 & 17 & & & & & 5000 & 7.7 & 4.4 & 3.7 & 17 & 1.53 & 0.51 & 4.8 & 1.1 & 15 \\
\hline 20 & 6.3 & 6.1 & 8.9 & & 5000 & 16.7 & 6.5 & 5.4 & 52 & & & & & 5000 & 16.7 & 6.5 & 5.4 & 52 & 2.03 & 0.83 & 9.3 & 2.6 & 20 \\
\hline 25 & 8.0 & 7.8 & 13.6 & 22.0 & 5000 & 25.4 & 8.0 & 7.1 & 103 & 1311 & 4.3 & 6.4 & 17 & 3689 & 21.2 & 8.5 & 7.1 & 87 & 1.45 & 1.02 & 9.3 & 4.1 & 25 \\
\hline 30 & 9.7 & 9.4 & 15.8 & 22.0 & 3689 & 27.8 & 9.8 & 8.6 & 133 & 1133 & 5.7 & 8.0 & 26 & 2556 & 22.1 & 10.5 & 8.7 & 107 & 1.22 & 1.07 & 9.0 & 5.0 & 30 \\
\hline 35 & $\mid 11.1$ & 10.9 & 17.9 & 22.0 & 2556 & 27.8 & 11.8 & 10.1 & 150 & 629 & 4.6 & 9.7 & 24 & 1927 & 23.1 & 12.4 & 10.2 & 126 & 1.04 & 1.08 & 8.5 & 5.5 & 35 \\
\hline 40 & 12.4 & 12.2 & 19.7 & 22.0 & 1927 & 27.9 & 13.6 & 11.4 & 167 & 386 & 3.9 & 11.3 & 22 & 1541 & 24.0 & 14.1 & 11.5 & 145 & 0.86 & 1.06 & 7.7 & 5.8 & 40 \\
\hline 45 & 13.6 & 13.3 & 21.3 & 22.0 & 1541 & 28.1 & 15.2 & 12.6 & 181 & 254 & 3.3 & 12.8 & 20 & 1287 & 24.8 & 15.7 & 12.7 & 161 & 0.76 & 1.03 & 7.2 & 6.0 & 45 \\
\hline 50 & 14.7 & 14.4 & 22.8 & 22.0 & 1287 & 28.4 & 16.8 & 13.6 & 196 & 177 & 2.8 & 14.3 & 19 & 1111 & 25.6 & 17.1 & 13.7 & 177 & 0.71 & 1.00 & 6.9 & 6.1 & 50 \\
\hline 55 & 15.6 & 15.3 & 24.3 & 22.6 & 1111 & 29.0 & 18.2 & 14.6 & 211 & 180 & 3.5 & 15.8 & 25 & 931 & 25.5 & 18.7 & 14.7 & 186 & 0.65 & 0.97 & 6.5 & 6.2 & 55 \\
\hline 60 & \begin{tabular}{|l|l}
16.4 \\
\end{tabular} & 16.1 & 25.7 & 23.2 & 931 & 28.6 & 19.8 & 15.4 & 217 & 134 & 3.2 & 17.4 & 23 & 797 & 25.4 & 20.1 & 15.5 & 194 & 0.60 & 0.95 & 6.1 & 6.2 & 60 \\
\hline 65 & $\mid 17.1$ & 16.8 & 27.0 & 23.8 & 797 & 28.3 & 21.2 & 16.2 & 223 & 103 & 2.9 & 19.0 & 22 & 694 & 25.3 & 21.6 & 16.3 & 201 & 0.55 & 0.92 & 5.7 & 6.2 & 65 \\
\hline 70 & \begin{tabular}{|l}
17.8 \\
\end{tabular} & 17.5 & 28.2 & 24.4 & 694 & 28.0 & 22.7 & 16.9 & 229 & 82 & 2.7 & 20.6 & 21 & 612 & 25.3 & 22.9 & 17.0 & 207 & 0.52 & 0.89 & 5.3 & 6.1 & 70 \\
\hline 75 & 18.4 & 18.1 & 29.4 & 25.0 & 612 & 27.8 & 24.0 & 17.5 & 233 & 66 & 2.5 & 22.1 & 20 & 546 & 25.3 & 24.3 & 17.6 & 213 & 0.48 & 0.86 & 5.0 & 6.0 & 75 \\
\hline 80 & $\mid 18.9$ & 18.6 & 30.6 & 25.6 & 546 & 27.6 & 25.4 & 18.1 & 237 & 54 & 2.4 & 23.6 & 20 & 492 & 25.3 & 25.6 & 18.2 & 218 & 0.46 & 0.84 & 4.8 & 6.0 & 80 \\
\hline 85 & \begin{tabular}{|l|l}
19.4 \\
\end{tabular} & 19.1 & 31.7 & 26.2 & 492 & 27.5 & 26.7 & 18.6 & 241 & 45 & 2.2 & 25.2 & 19 & 447 & 25.2 & 26.8 & 18.7 & 222 & 0.43 & 0.82 & 4.5 & 5.9 & 85 \\
\hline 90 & \begin{tabular}{|l}
19.8 \\
\end{tabular} & 19.5 & 32.9 & 26.8 & 447 & 27.3 & 27.9 & 19.0 & 244 & 38 & 1.7 & 24.1 & 15 & 409 & 25.6 & 28.2 & 19.2 & 229 & 0.41 & 0.79 & 4.3 & 5.8 & 90 \\
\hline 95 & 20.2 & 19.9 & 34.2 & 27.4 & 409 & 27.6 & 29.3 & 19.5 & 250 & 33 & 1.7 & 25.4 & 14 & 376 & 25.9 & 29.6 & 19.6 & 235 & 0.39 & 0.77 & 4.1 & 5.7 & 95 \\
\hline 100 & 20.6 & 20.2 & 35.4 & 28.0 & 376 & 27.8 & 30.7 & 19.9 & 256 & 28 & 1.6 & 26.6 & 14 & 348 & 26.3 & 31.0 & 20.0 & 242 & 0.37 & 0.75 & 3.9 & 5.6 & 100 \\
\hline 105 & 20.9 & 20.5 & 36.6 & 28.6 & 348 & 28.1 & 32.1 & 20.2 & 261 & 25 & 1.5 & 27.7 & J & 323 & 26.6 & 32.4 & 20.4 & 247 & 0.36 & 0.73 & 3.7 & 5.6 & 105 \\
\hline 110 & 21.2 & 20.8 & 37.8 & 29.2 & 323 & 28.3 & 33.4 & 20.6 & 266 & 22 & 1.4 & 28.9 & 13 & 302 & 26.9 & 33.7 & 20.7 & 253 & 0.34 & 0.72 & 3.6 & 5.5 & 110 \\
\hline 115 & 21.5 & 21.1 & 38.9 & 29.8 & 302 & 28.6 & 34.7 & 20.9 & 271 & 19 & 1.4 & 30.1 & 12 & 282 & 27.2 & 35.0 & 21.0 & 258 & 0.33 & 0.70 & 3.4 & 5.4 & 115 \\
\hline 120 & 21.7 & 21.3 & 40.1 & 30.4 & 282 & 28.8 & 36.1 & 21.2 & 275 & 17 & 1.3 & 31.2 & 12 & 265 & 27.5 & 36.4 & 21.3 & 263 & 0.32 & 0.69 & 3.3 & 5.3 & 120 \\
\hline 125 & 21.9 & 21.6 & 41.2 & 31.0 & 265 & 29.1 & 37.4 & 21.4 & 279 & 15 & 1.3 & 32.3 & 12 & 250 & 27.8 & 37.7 & 21.6 & 267 & 0.31 & 0.67 & 3.2 & 5.2 & 125 \\
\hline 130 & 22.1 & 21.8 & 42.3 & 31.6 & 250 & 29.3 & 38.7 & 21.7 & 283 & 14 & 1.2 & 33.4 & 11 & 236 & 28.1 & 38.9 & 21.9 & 272 & 0.29 & 0.66 & 3.1 & 5.1 & 130 \\
\hline 135 & 22.3 & 22.0 & 43.5 & 32.2 & 236 & 29.5 & 39.9 & 21.9 & 287 & 13 & 1.2 & 34.5 & 11 & 223 & 28.4 & 40.2 & 22.1 & 276 & 0.29 & 0.64 & 3.0 & 5.1 & 135 \\
\hline 140 & 22.5 & 22.2 & 44.6 & 32.8 & 223 & 29.8 & 41.2 & 22.2 & 290 & 12 & 1.1 & 35.6 & 11 & 212 & 28.6 & 41.5 & 22.3 & 280 & 0.28 & 0.63 & 2.9 & 5.0 & 140 \\
\hline 145 & 22.7 & 22.4 & 45.7 & 33.4 & 212 & 30.0 & 42.5 & 22.4 & 294 & 11 & 1.1 & 36.7 & 11 & 201 & 28.8 & 42.7 & 22.5 & 283 & 0.27 & 0.62 & 2.8 & 4.9 & 145 \\
\hline 150 & 22.8 & 22.6 & 46.8 & 34.0 & 201 & 30.2 & 43.7 & 22.6 & 297 & 10 & 1.1 & 37.8 & 10 & 191 & 29.1 & 44.0 & 22.7 & 286 & 0.26 & 0.61 & 2.7 & 4.8 & 150 \\
\hline
\end{tabular}




\begin{tabular}{|c|c|c|c|c|c|c|c|c|c|c|c|c|c|c|c|c|c|c|c|c|c|c|c|}
\hline \multirow{2}{*}{\multicolumn{5}{|c|}{$\begin{array}{l}\text { GROVE DEN, Nederland } 2018 \\
\text { SCOTS PINE, Netherlands } 2018 \\
\end{array}$}} & \multicolumn{15}{|c|}{ sterke dunning } & \multicolumn{4}{|c|}{ Boniteit IV, $h_{70}=14.8$} \\
\hline & & & & & & & & & & & & eavy $t$ & ning & & & & & & & & ite $\mathrm{Cl}$ & s IV, $h$ & $=14.8$ \\
\hline \multirow[b]{2}{*}{$t$} & \multicolumn{4}{|c|}{$\begin{array}{l}\text { Opstandkenmerken } \\
\text { Stand characteristics }\end{array}$} & \multicolumn{5}{|c|}{$\begin{array}{l}\text { Kenmerken voor dunning } \\
\text { Characteristics before thinning }\end{array}$} & \multicolumn{4}{|c|}{$\begin{array}{l}\text { Dunning } \\
\text { Thinning }\end{array}$} & \multicolumn{5}{|c|}{$\begin{array}{c}\text { Kenmerken na dunning } \\
\text { Characteristics after thinning }\end{array}$} & \multicolumn{4}{|c|}{$\begin{array}{l}\text { Bijgroei } \\
\text { Increment }\end{array}$} & \multirow[b]{2}{*}{$t$} \\
\hline & $h_{\text {top }}$ & $\boldsymbol{h}_{d o m}$ & $\boldsymbol{d}_{\text {dom }}$ & S\% & $N$ & $G$ & $d_{g}$ & $h_{g}$ & $v$ & $N$ & $\boldsymbol{G}$ & $d_{g}$ & $v$ & $N$ & $G$ & $d_{g}$ & $\boldsymbol{h}_{g}$ & $v$ & $I c_{G}$ & $I m_{G}$ & $I c_{v}$ & $I m_{V}$ & \\
\hline 5 & 0.6 & 0.6 & & & 5000 & & & & 0 & & & & & 5000 & & & & 0 & & & 0.0 & 0.0 & 5 \\
\hline 10 & 1.8 & 1.7 & 1.2 & & 5000 & 0.3 & 0.9 & 1.4 & 0 & & & & & 5000 & 0.3 & 0.9 & 1.4 & 0 & 0.28 & 0.03 & 0.3 & 0.0 & 10 \\
\hline 15 & 3.1 & 2.9 & 3.8 & & 5000 & 3.0 & 2.8 & 2.5 & 5 & & & & & 5000 & 3.0 & 2.8 & 2.5 & 5 & 0.76 & 0.20 & 1.6 & 0.3 & 15 \\
\hline 20 & 4.5 & 4.3 & 6.1 & & 5000 & 7.8 & 4.5 & 3.7 & 17 & & & & & 5000 & 7.8 & 4.5 & 3.7 & 17 & 1.14 & 0.39 & 3.6 & 0.9 & 20 \\
\hline 25 & 5.8 & 5.7 & 8.3 & & 5000 & 14.3 & 6.0 & 5.0 & 41 & & & & & 5000 & 14.3 & 6.0 & 5.0 & 41 & 1.42 & 0.57 & 6.0 & 1.7 & 25 \\
\hline 30 & 7.2 & 7.0 & 12.4 & 21.2 & 5000 & 21.7 & 7.4 & 6.3 & 77 & & & & & 5000 & 21.7 & 7.4 & 6.3 & 77 & 1.37 & 0.72 & 8.8 & 2.6 & 30 \\
\hline 35 & 8.4 & 8.2 & 14.4 & 22.0 & 5000 & 27.9 & 8.4 & 7.5 & 117 & 1634 & 6.0 & 6.8 & 24 & 3366 & 21.9 & 9.1 & 7.5 & 93 & 1.16 & 0.80 & 7.4 & 3.4 & 35 \\
\hline 40 & 9.6 & 9.3 & 16.1 & 22.0 & 3366 & 27.4 & 10.2 & 8.6 & 129 & 768 & 4.2 & 8.3 & 19 & 2598 & 23.2 & 10.7 & 8.7 & 110 & 1.02 & 0.83 & 7.2 & 3.8 & 40 \\
\hline 45 & 10.7 & 10.4 & 17.8 & 22.0 & 2598 & 28.0 & 11.7 & 9.7 & 145 & 497 & 3.7 & 9.7 & 18 & 2101 & 24.3 & 12.1 & 9.7 & 127 & 0.91 & 0.85 & 6.9 & 4.2 & 45 \\
\hline 50 & 11.6 & 11.4 & 19.3 & 22.0 & 2101 & 28.6 & 13.2 & 10.6 & 160 & 341 & 3.3 & 11.0 & 18 & 1760 & 25.3 & 13.5 & 10.7 & 143 & 0.81 & 0.85 & 6.5 & 4.4 & 50 \\
\hline 55 & 12.5 & 12.3 & 20.7 & 22.6 & 1760 & 29.1 & 14.5 & 11.6 & 174 & 323 & 3.9 & 12.4 & 22 & 1437 & 25.2 & 15.0 & 11.6 & 152 & 0.68 & 0.84 & 5.9 & 4.6 & 55 \\
\hline 60 & \begin{tabular}{|l|}
13.4 \\
\end{tabular} & 13.1 & 22.0 & 23.2 & 1437 & 28.5 & 15.9 & 12.4 & 180 & 236 & 3.5 & 13.8 & 21 & 1201 & 24.9 & 16.3 & 12.5 & 159 & 0.62 & 0.82 & 5.5 & 4.7 & 60 \\
\hline 65 & \begin{tabular}{|l|}
14.1 \\
\end{tabular} & 13.8 & 23.2 & 23.8 & 1201 & 27.9 & 17.2 & 13.2 & 185 & 178 & 3.2 & 15.1 & 20 & 1023 & 24.7 & 17.5 & 13.3 & 165 & 0.57 & 0.81 & 5.2 & 4.7 & 65 \\
\hline 70 & \begin{tabular}{|l|l}
14.8 \\
\end{tabular} & 14.5 & 24.3 & 24.4 & 1023 & 27.5 & 18.5 & 13.9 & 190 & 138 & 2.9 & 16.5 & 20 & 886 & 24.6 & 18.8 & 14.0 & 171 & 0.54 & 0.79 & 4.9 & 4.8 & 70 \\
\hline 75 & \begin{tabular}{|l|}
15.4 \\
\end{tabular} & 15.1 & 25.5 & 25.0 & 886 & 27.1 & 19.8 & 14.5 & 195 & 109 & 2.7 & 17.9 & 19 & 777 & 24.4 & 20.0 & 14.6 & 176 & 0.50 & 0.77 & 4.7 & 4.8 & 75 \\
\hline 80 & 16.0 & 15.7 & 26.6 & 25.6 & 777 & 26.8 & 21.0 & 15.1 & 199 & 88 & 2.6 & 19.2 & 18 & 689 & 24.3 & 21.2 & 15.2 & 180 & 0.47 & 0.75 & 4.5 & 4.8 & 80 \\
\hline 85 & 16.5 & 16.2 & 27.6 & 26.2 & 689 & 26.6 & 22.2 & 15.7 & 202 & 72 & 2.4 & 20.6 & 18 & 617 & 24.2 & 22.3 & 15.8 & 185 & 0.45 & 0.74 & 4.2 & 4.7 & 85 \\
\hline 90 & \begin{tabular}{|l|l}
17.0 \\
\end{tabular} & 16.7 & 28.8 & 26.8 & 617 & 26.3 & 23.3 & 16.2 & 205 & 60 & 1.9 & 20.2 & 14 & 557 & 24.4 & 23.6 & 16.3 & 191 & 0.42 & 0.72 & 4.0 & 4.7 & 90 \\
\hline 95 & \begin{tabular}{|l|}
17.4 \\
\end{tabular} & 17.1 & 29.9 & 27.4 & 557 & 26.5 & 24.6 & 16.7 & 211 & 51 & 1.8 & 21.3 & 14 & 506 & 24.7 & 24.9 & 16.8 & 197 & 0.40 & 0.70 & 3.9 & 4.7 & 95 \\
\hline 100 & \begin{tabular}{|l|}
17.8 \\
\end{tabular} & 17.5 & 31.0 & 28.0 & 506 & 26.6 & 25.9 & 17.1 & 216 & 43 & 1.7 & 22.4 & 13 & 463 & 24.9 & 26.2 & 17.2 & 203 & 0.38 & 0.69 & 3.7 & 4.6 & 100 \\
\hline 105 & \begin{tabular}{|l|}
18.2 \\
\end{tabular} & 17.9 & 32.2 & 28.6 & 463 & 26.8 & 27.2 & 17.5 & 221 & 37 & 1.6 & 23.5 & 13 & 426 & 25.2 & 27.5 & 17.6 & 208 & 0.37 & 0.67 & 3.6 & 4.6 & 105 \\
\hline 110 & 18.5 & 18.2 & 33.3 & 29.2 & 426 & 27.0 & 28.4 & 17.9 & 225 & 32 & 1.5 & 24.6 & 12 & 394 & 25.5 & 28.7 & 18.0 & 213 & 0.35 & 0.66 & 3.4 & 4.5 & 110 \\
\hline 115 & \begin{tabular}{|l}
18.9 \\
\end{tabular} & 18.5 & 34.3 & 29.8 & 394 & 27.2 & 29.7 & 18.2 & 230 & 28 & 1.5 & 25.7 & 12 & 365 & 25.7 & 29.9 & 18.4 & 218 & 0.34 & 0.64 & 3.3 & 4.5 & 115 \\
\hline 120 & \begin{tabular}{|l|}
19.2 \\
\end{tabular} & 18.8 & 35.4 & 30.4 & 365 & 27.4 & 30.9 & 18.6 & 234 & 25 & 1.4 & 26.7 & 12 & 341 & 26.0 & 31.2 & 18.7 & 223 & 0.32 & 0.63 & 3.2 & 4.4 & 120 \\
\hline 125 & \begin{tabular}{|l|}
19.4 \\
\end{tabular} & 19.1 & 36.5 & 31.0 & 341 & 27.6 & 32.1 & 18.9 & 238 & 22 & 1.3 & 27.8 & 11 & 318 & 26.2 & 32.4 & 19.0 & 227 & 0.31 & 0.62 & 3.1 & 4.4 & 125 \\
\hline 130 & \begin{tabular}{|l|}
19.7 \\
\end{tabular} & 19.3 & 37.6 & 31.6 & 318 & 27.8 & 33.3 & 19.2 & 242 & 20 & 1.3 & 28.8 & 11 & 299 & 26.5 & 33.6 & 19.3 & 231 & 0.30 & 0.61 & 3.0 & 4.3 & 130 \\
\hline 135 & 19.9 & 19.6 & 38.6 & 32.2 & 299 & 28.0 & 34.5 & 19.5 & 246 & 18 & 1.2 & 29.9 & 11 & 281 & 26.7 & 34.8 & 19.6 & 235 & 0.29 & 0.60 & 2.9 & 4.3 & 135 \\
\hline 140 & 20.1 & 19.8 & 39.6 & 32.8 & 281 & 28.2 & 35.7 & 19.7 & 249 & 16 & 1.2 & 30.9 & 10 & 265 & 27.0 & 36.0 & 19.9 & 239 & 0.28 & 0.58 & 2.8 & 4.2 & 140 \\
\hline 145 & 20.3 & 20.0 & 40.7 & 33.4 & 265 & 28.4 & 36.9 & 20.0 & 253 & 15 & 1.2 & 31.9 & 10 & 250 & 27.2 & 37.2 & 20.1 & 243 & 0.27 & 0.57 & 2.7 & 4.2 & 145 \\
\hline 150 & 20.5 & 20.2 & 41.7 & 34.0 & 250 & 28.5 & 38.1 & 20.2 & 256 & 13 & 1.1 & 32.9 & 10 & 237 & 27.4 & 38.4 & 20.3 & 246 & 0.27 & 0.56 & 2.6 & 4.1 & 150 \\
\hline
\end{tabular}




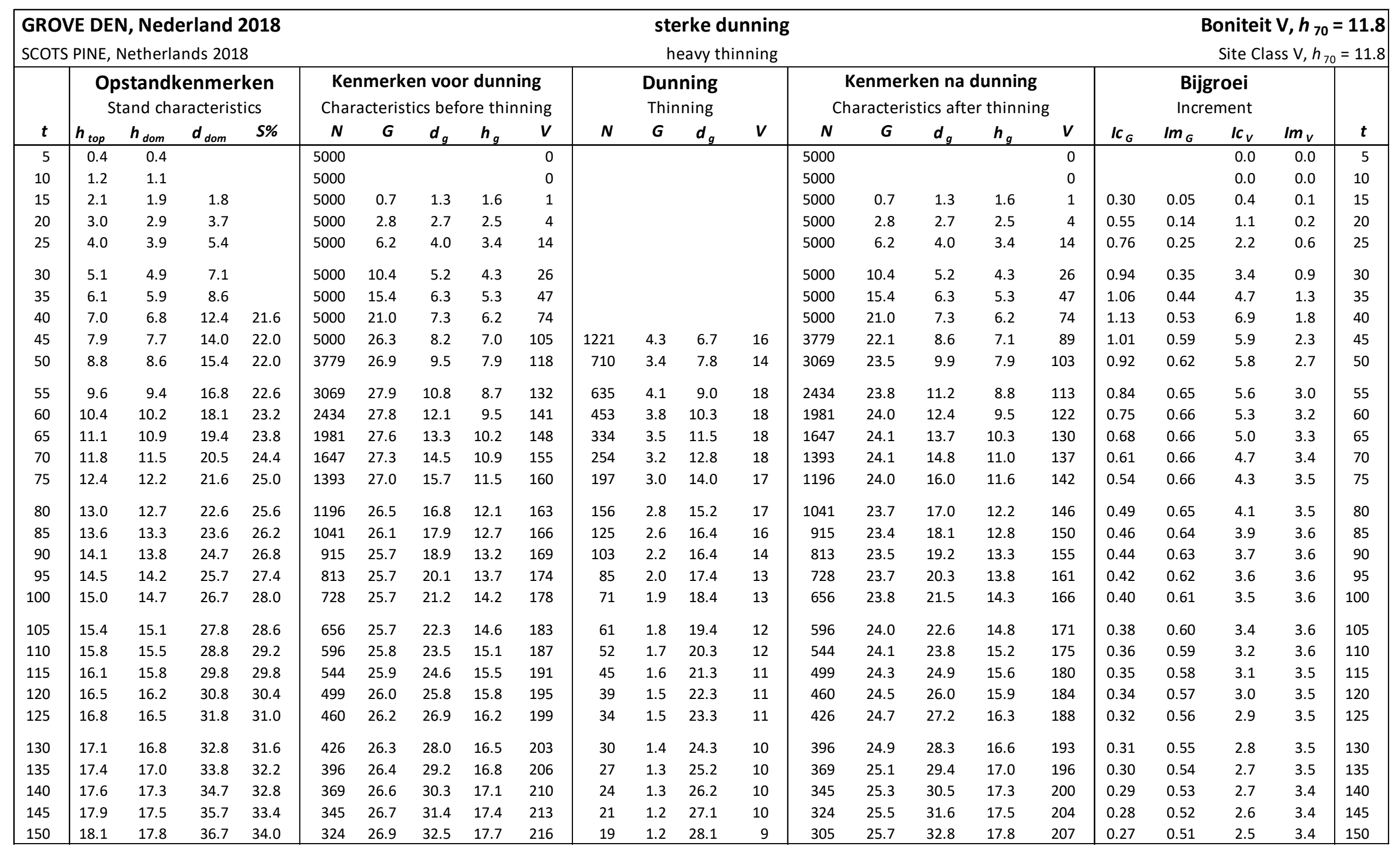




\section{Corsicaanse den (Pinus nigra subsp. laricio) Corsican pine} Jansen, J.J., A. Oosterbaan, G.M.J. Mohren en J. den Ouden

Bron: Jansen, J.J., A. Oosterbaan, G.M.J. Mohren en J. den Ouden, 2018. Groei en productie van Corsicaanse den in Nederland. FEM Groei en Productie Rapport 2018 - 6, 109 blz.

Dit rapport is gratis te downloaden op:

https://doi.org/10.18174/444095

N.B. In het Binnenland is de range van boniteiten voornamelijk I tot en met $V$. In het Kustgebied betreft dat de boniteiten III tot en met VII!

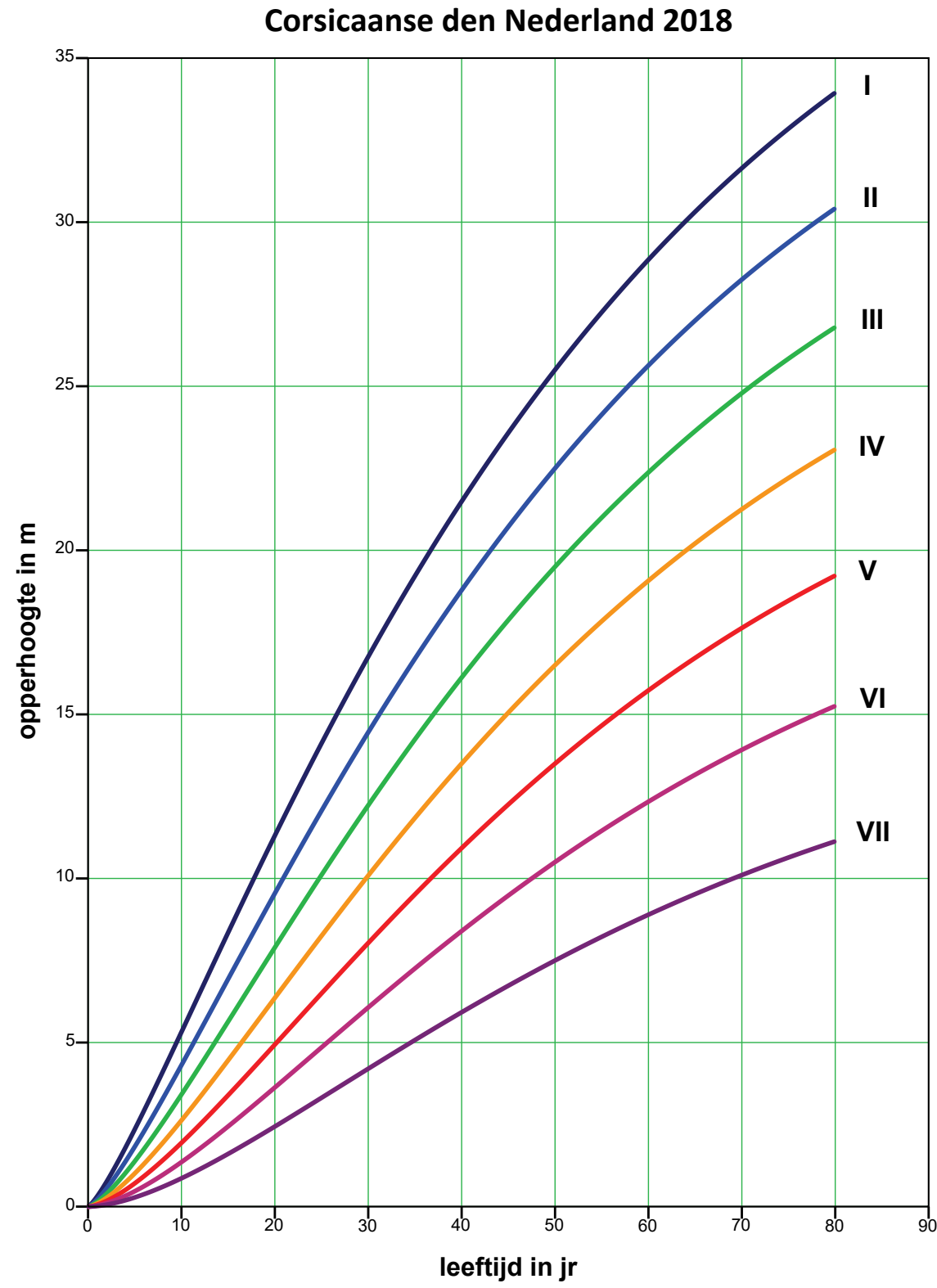




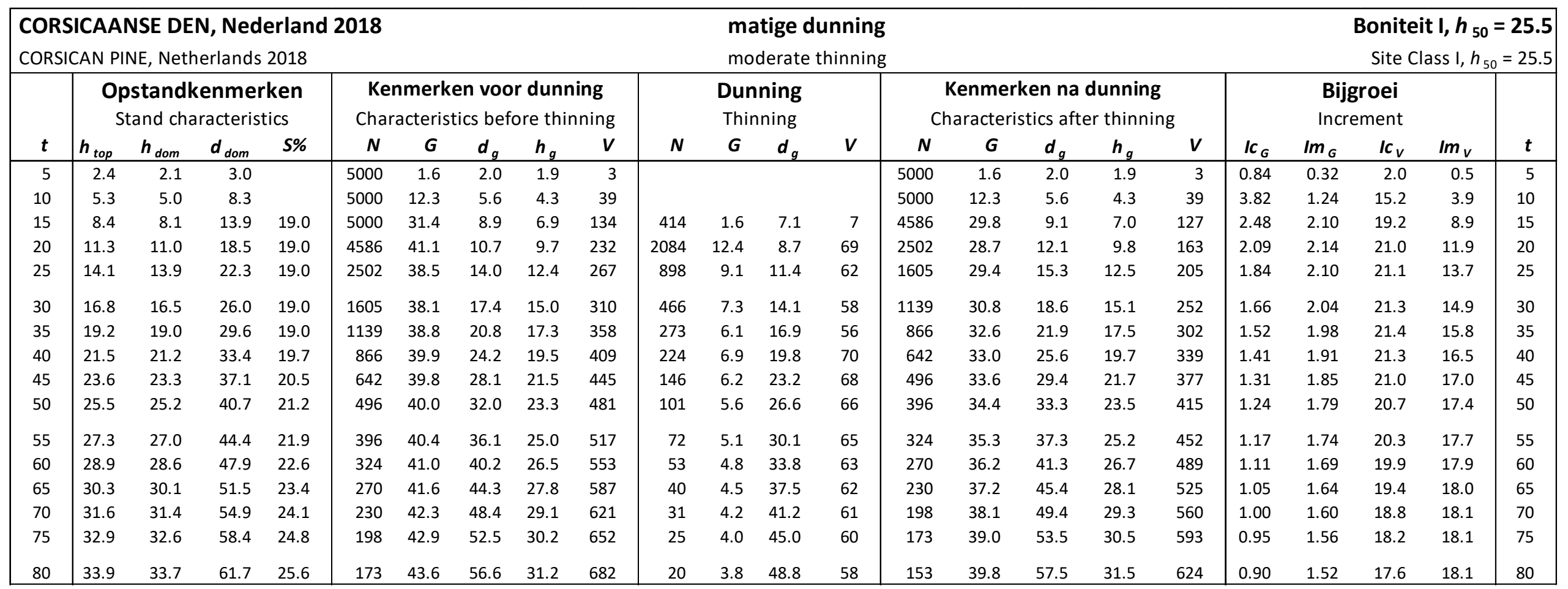




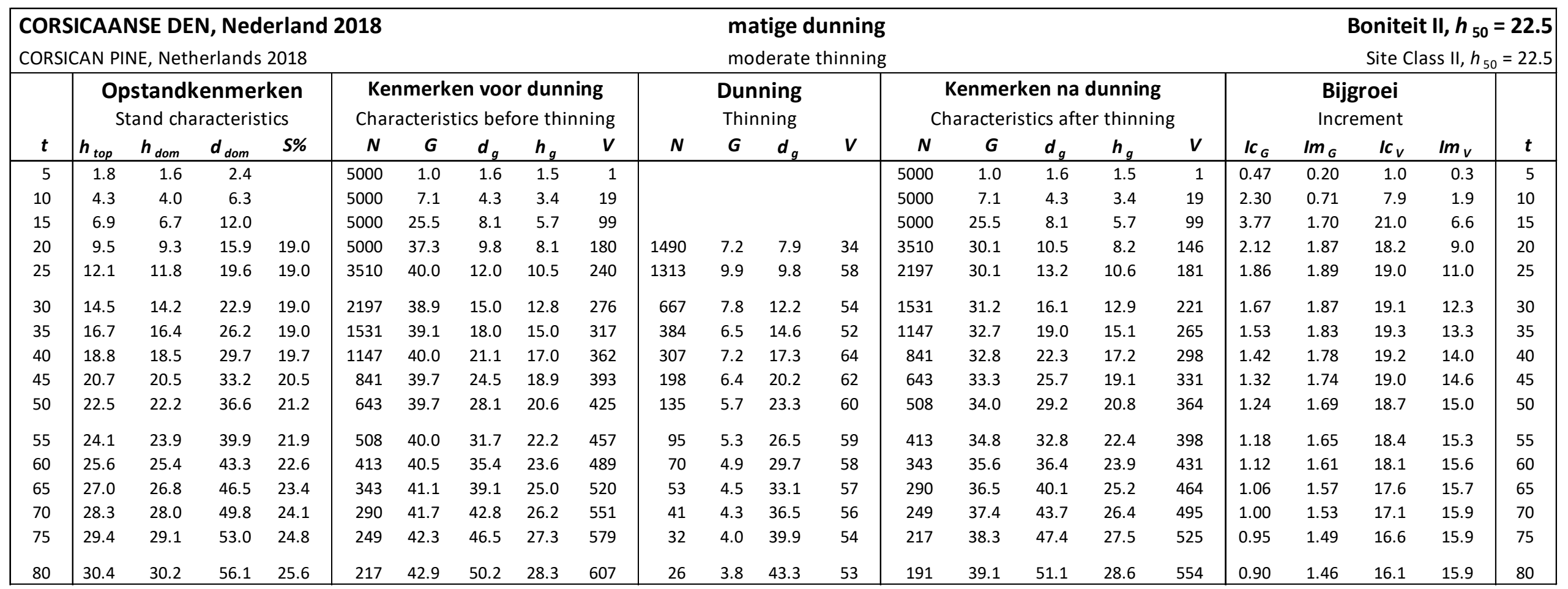




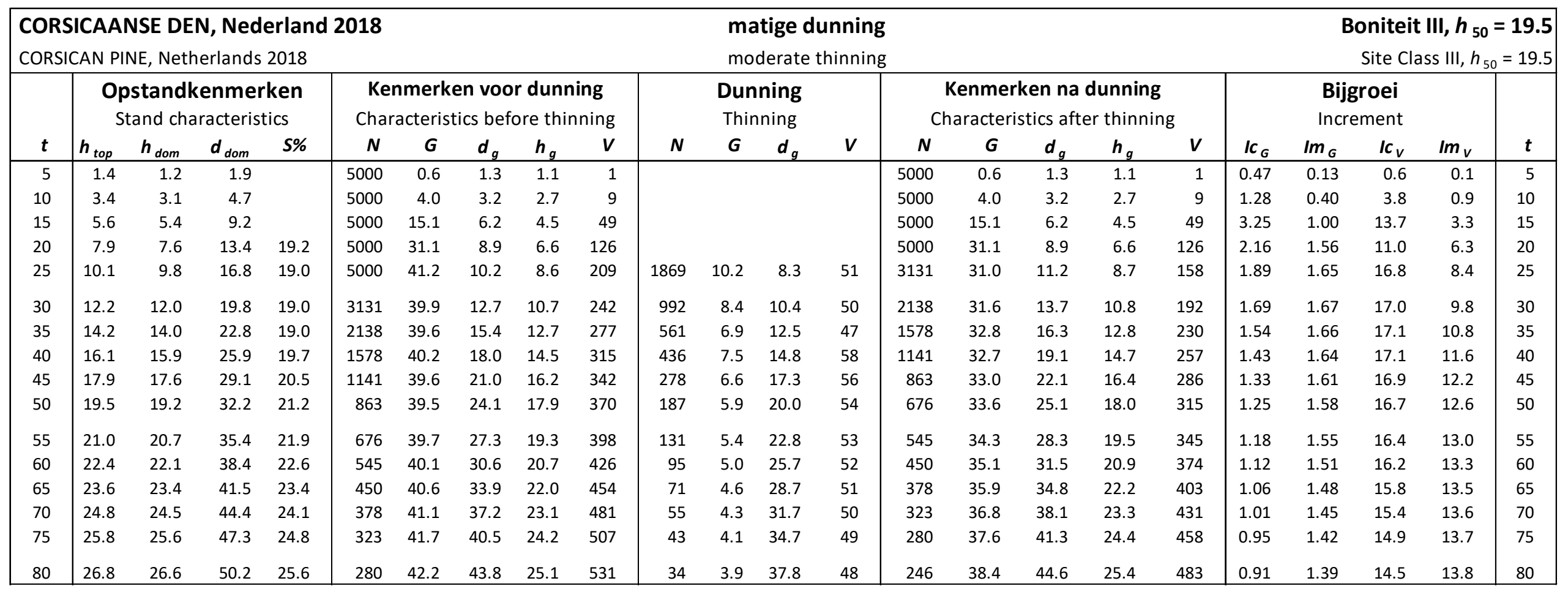




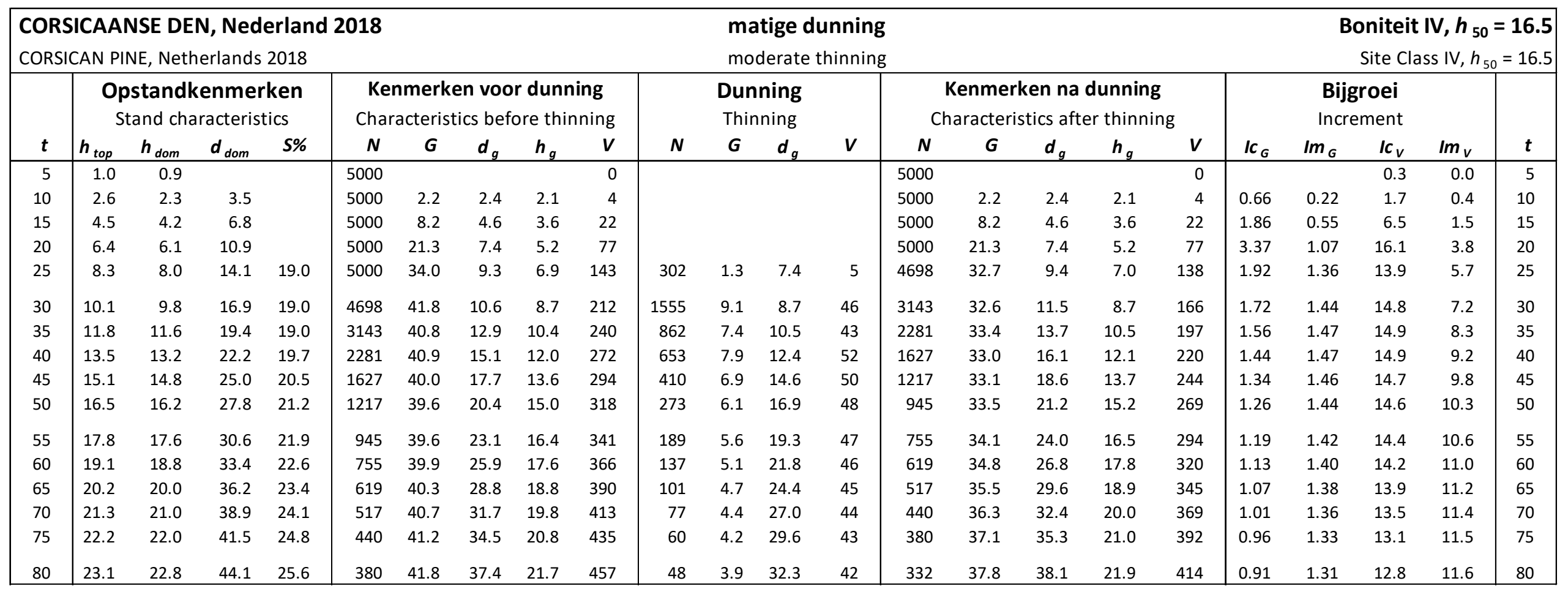




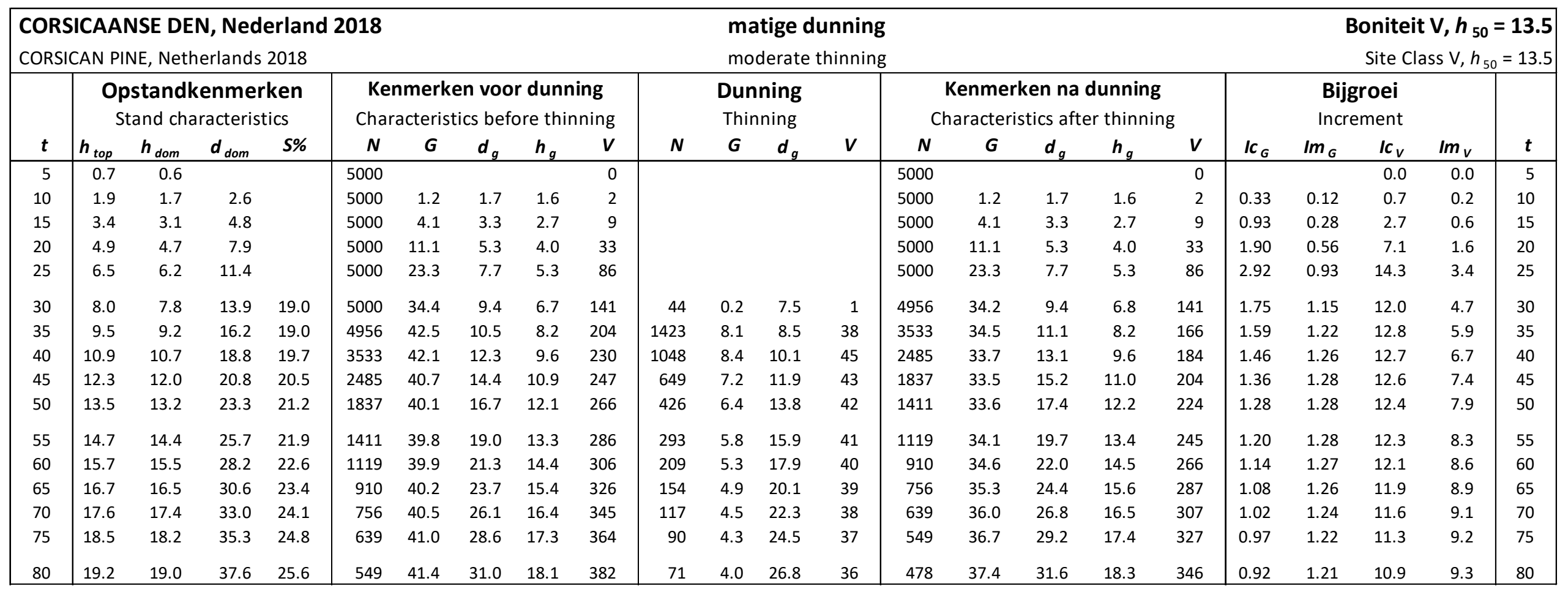




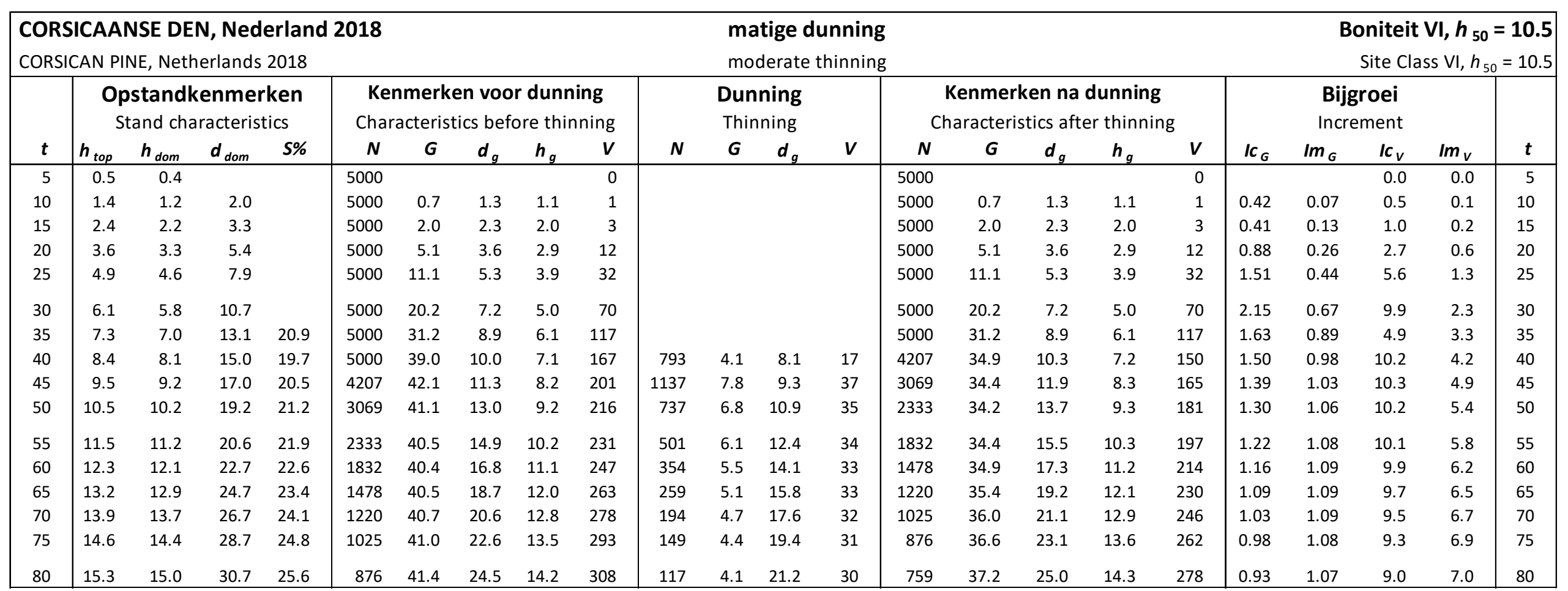




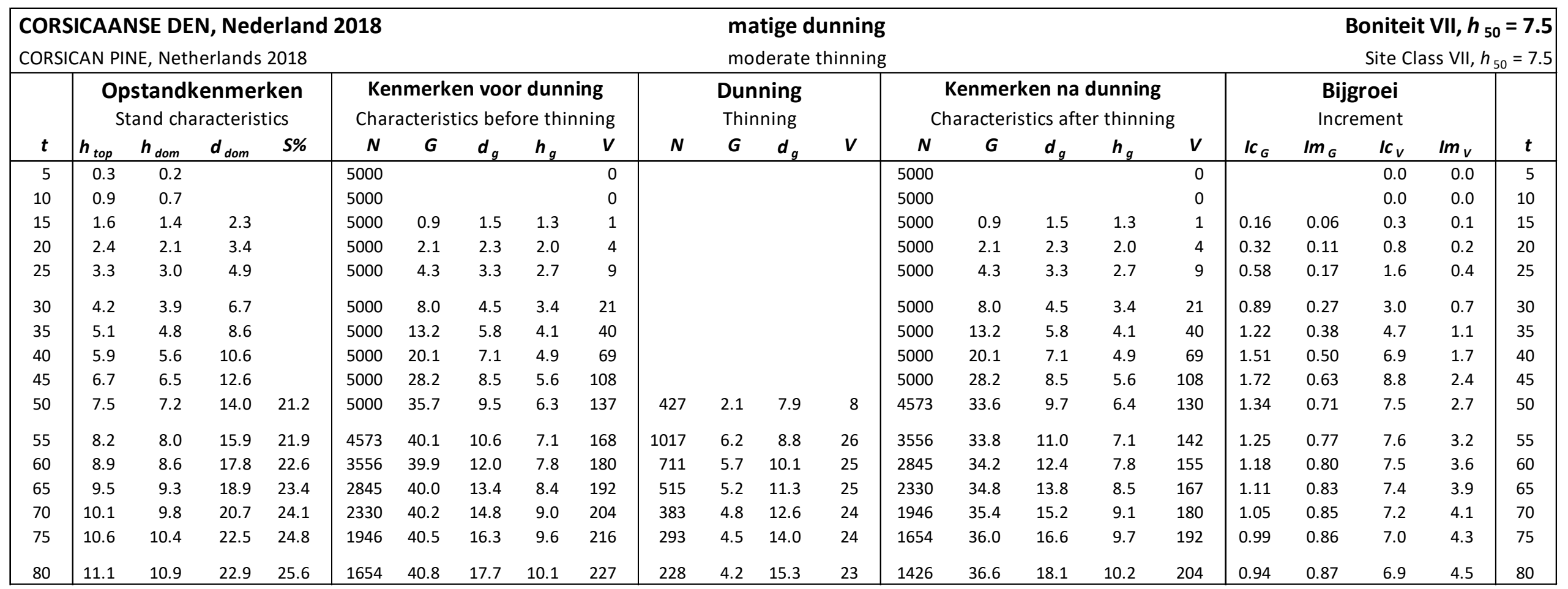




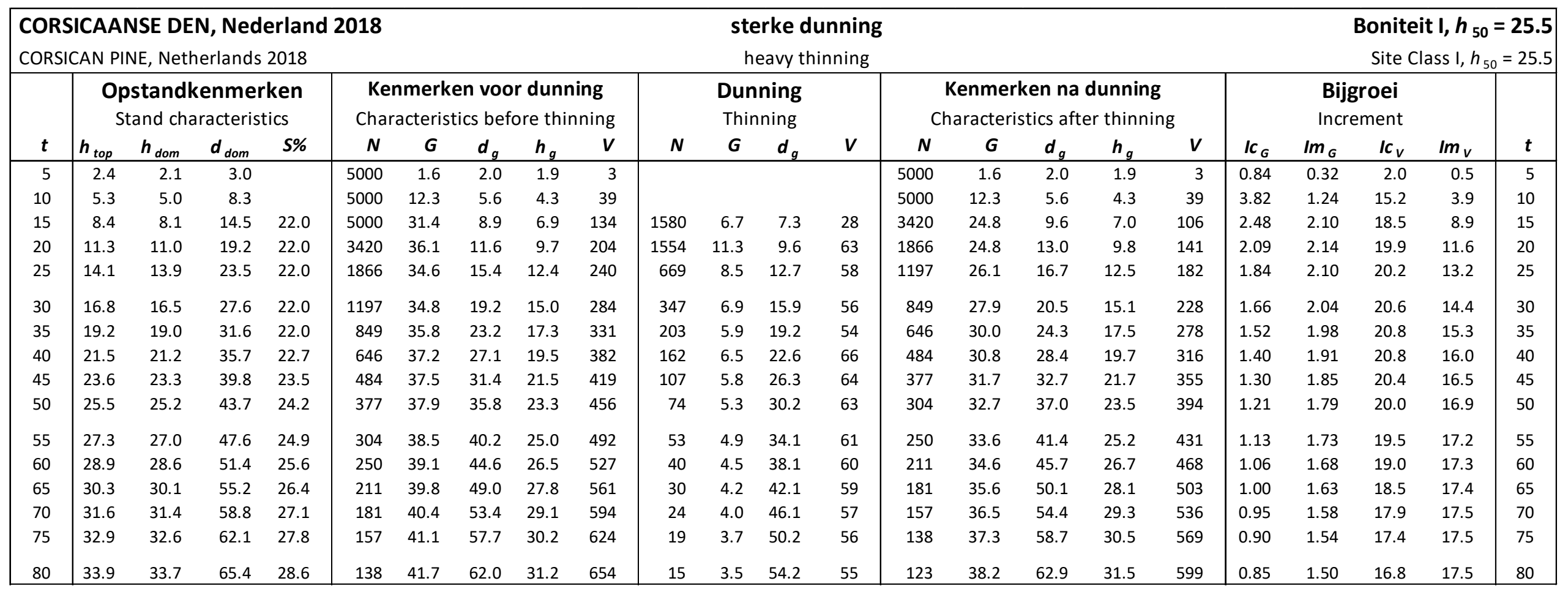




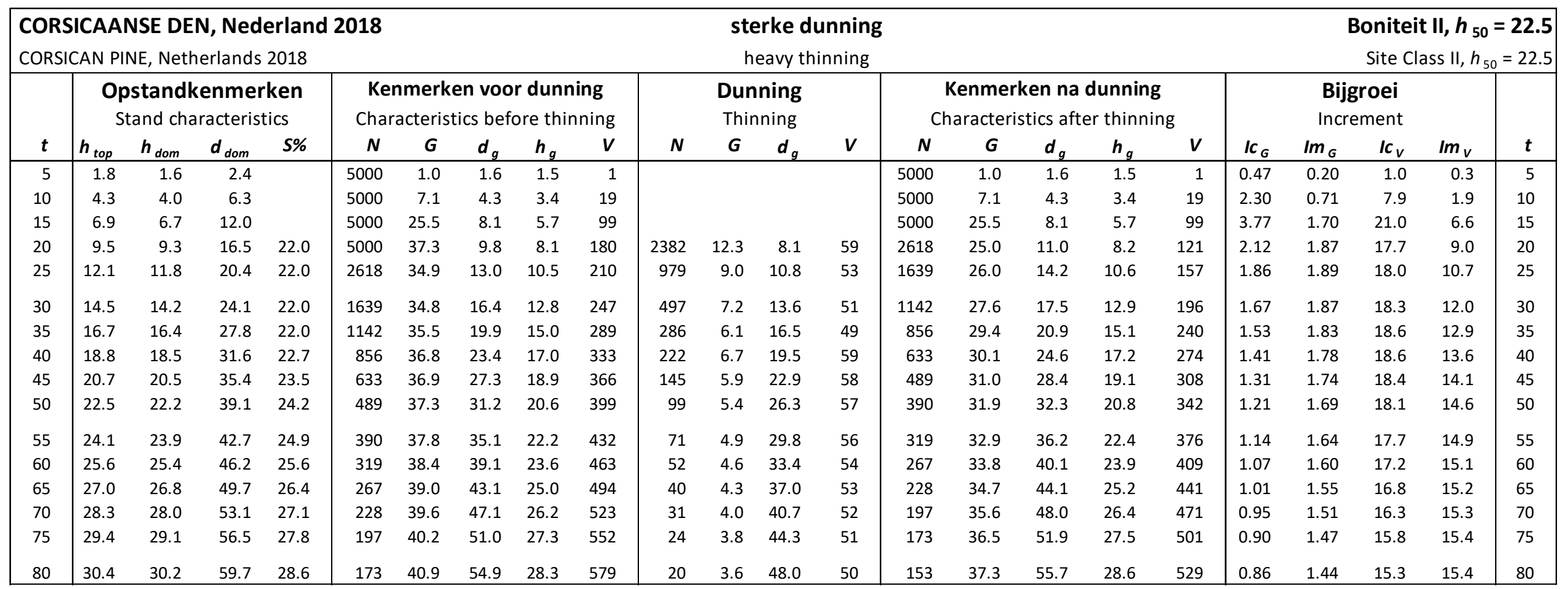




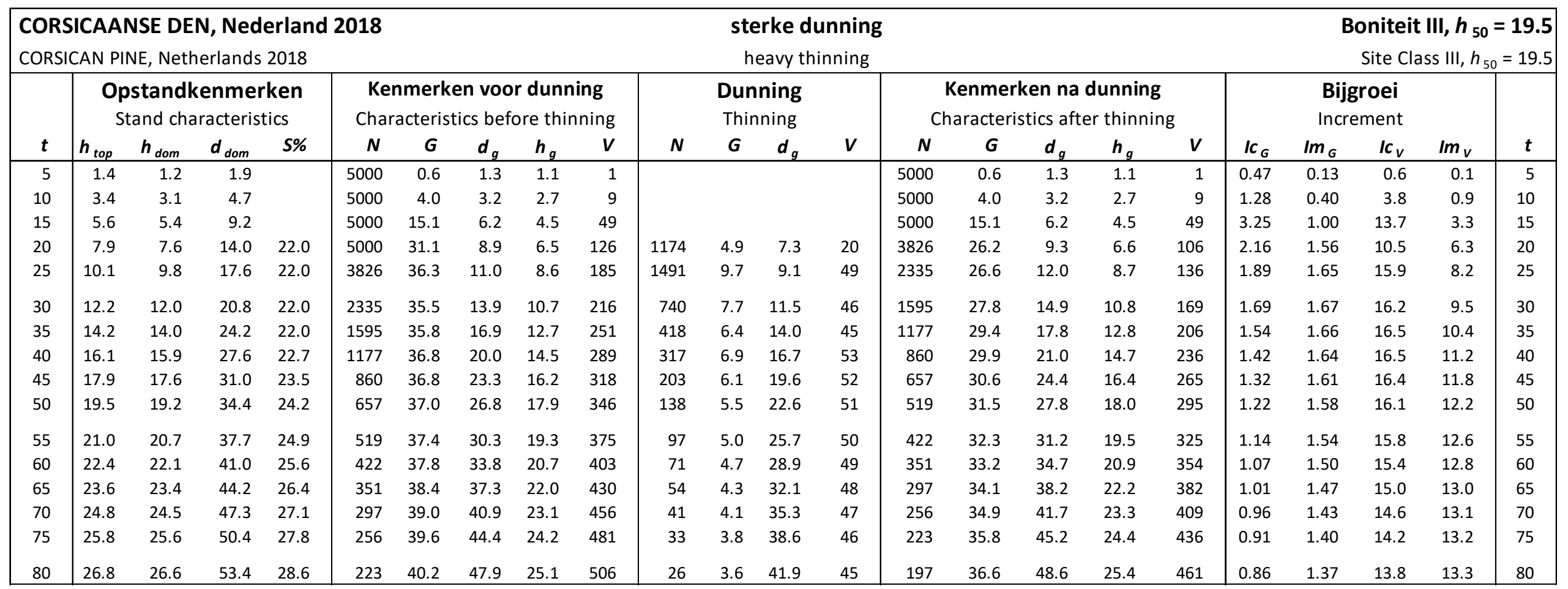




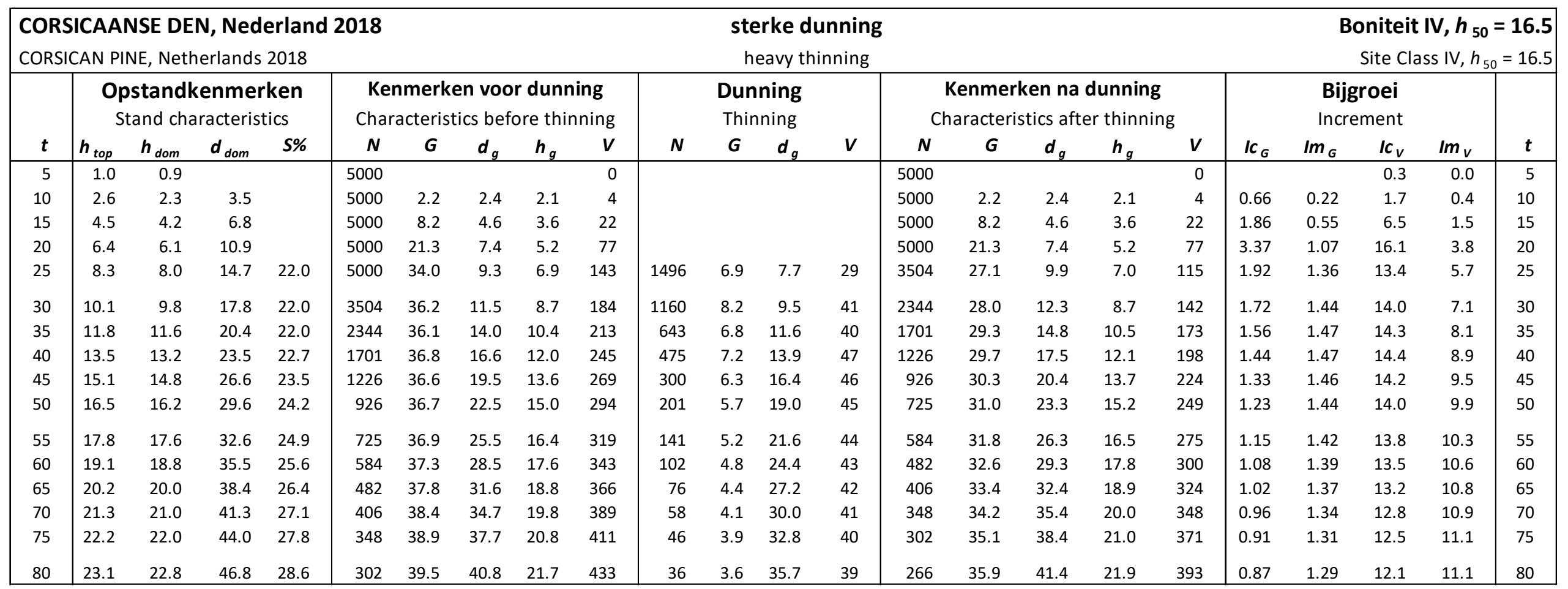




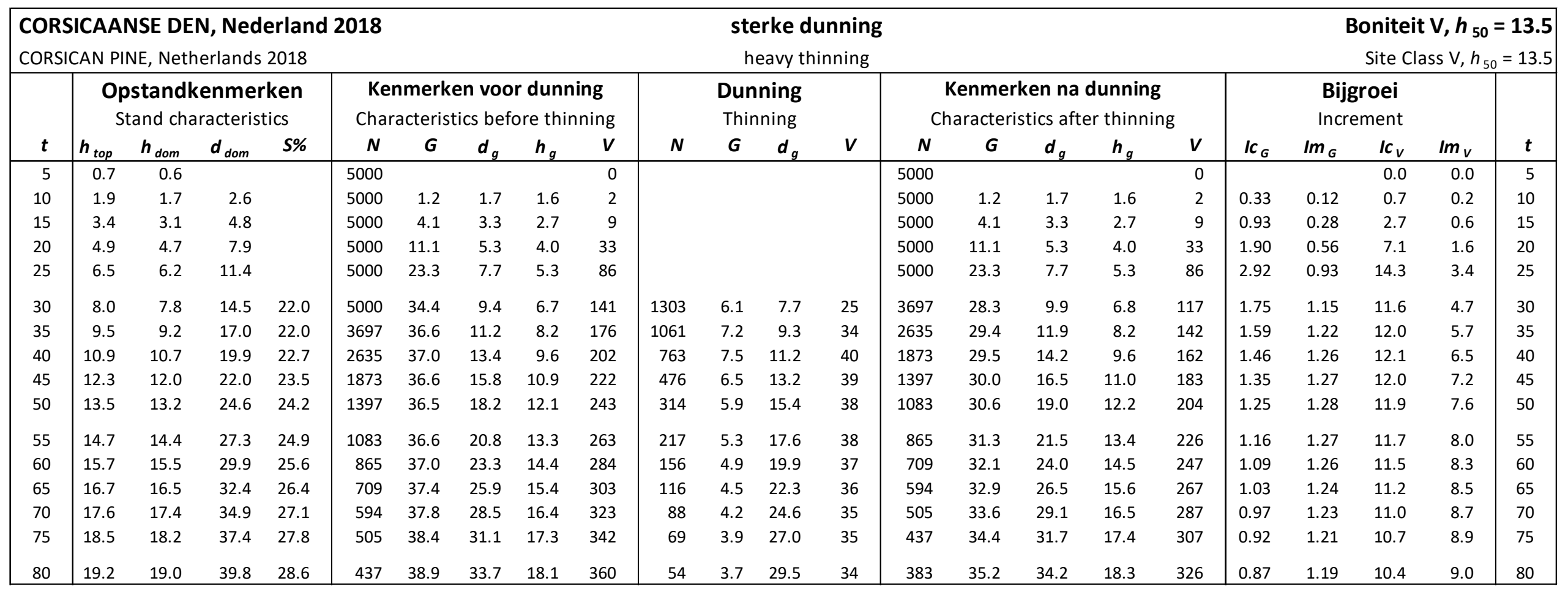




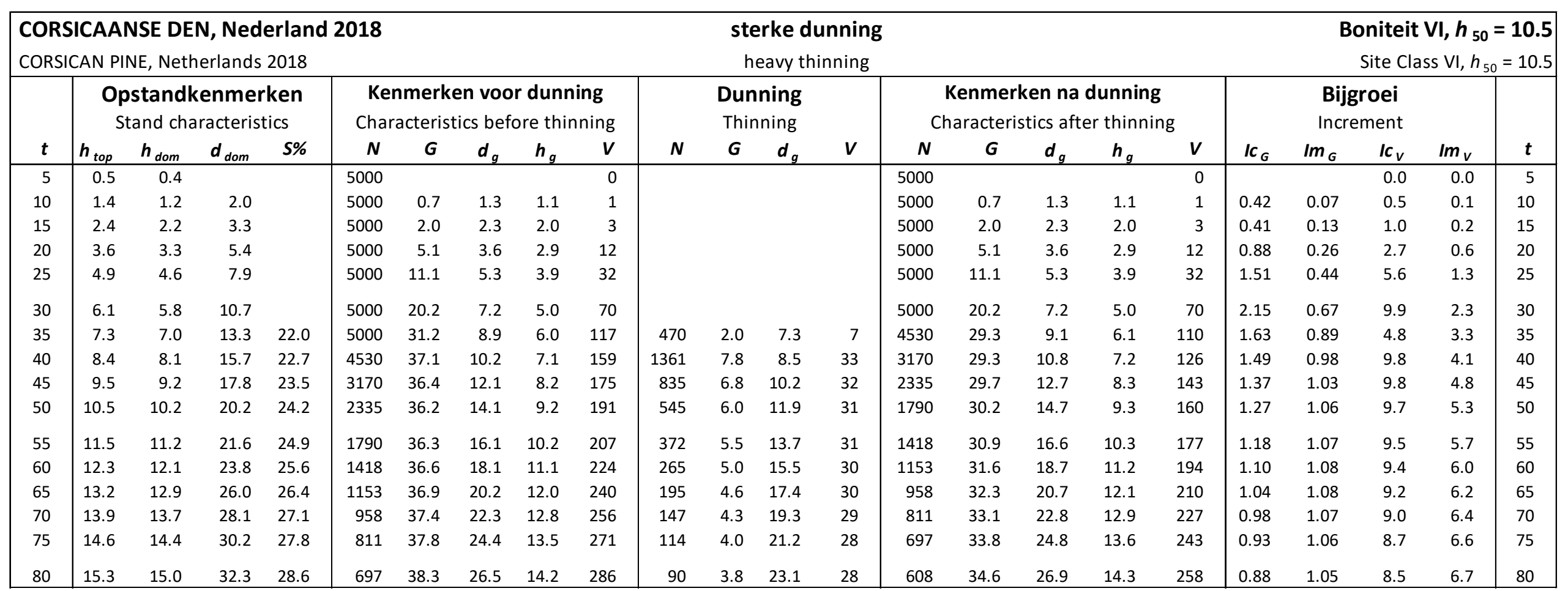




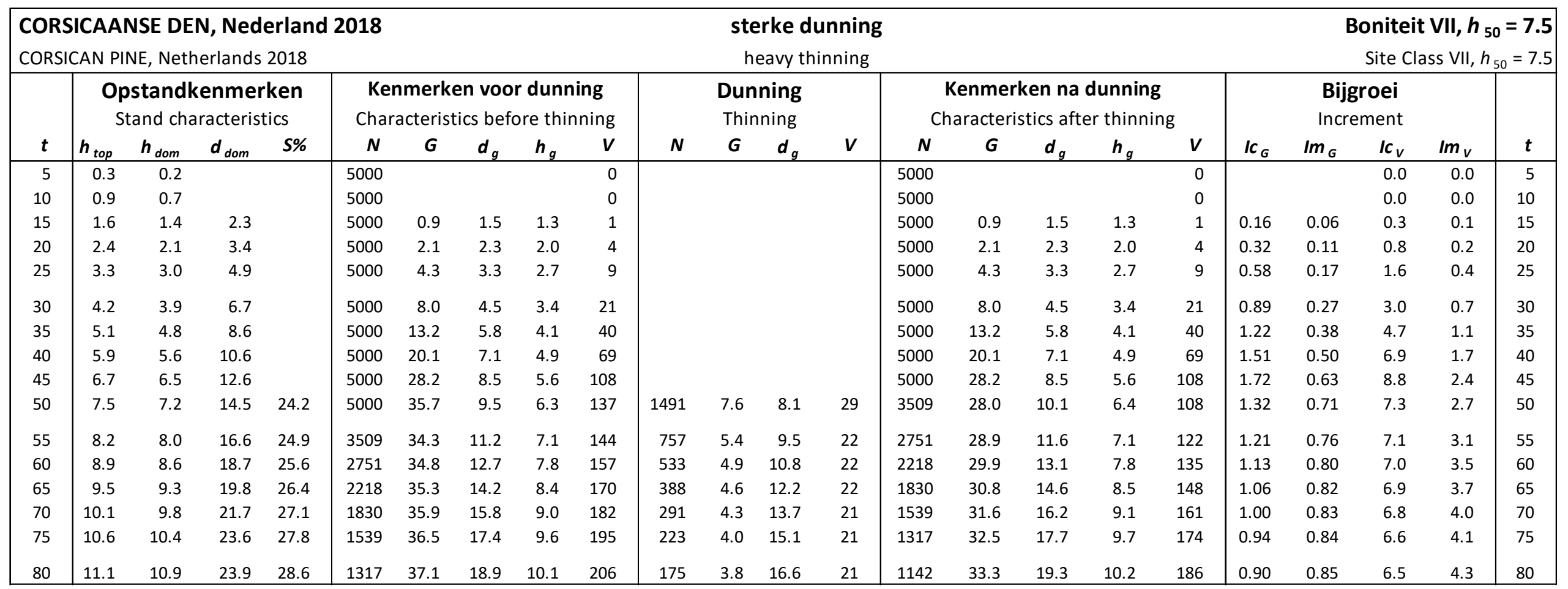




\section{Oostenrijkse den (Pinus nigra subsp. nigra) Austrian pine} Jansen, J.J., A. Oosterbaan, G.M.J. Mohren en J. den Ouden

Bron: Jansen, J.J., A. Oosterbaan, G.M.J. Mohren en J. den Ouden, 2018. Groei en productie van Oostenrijkse den in Nederland. FEM Groei en Productie Rapport 2018 - 7, 96 blz.

Dit rapport is gratis te downloaden op:

https://doi.org/10.18174/444096

N.B. In het Binnenland is de range van boniteiten voornamelijk I tot en met $V$. In het Kustgebied betreft dat de boniteiten III tot en met VII!

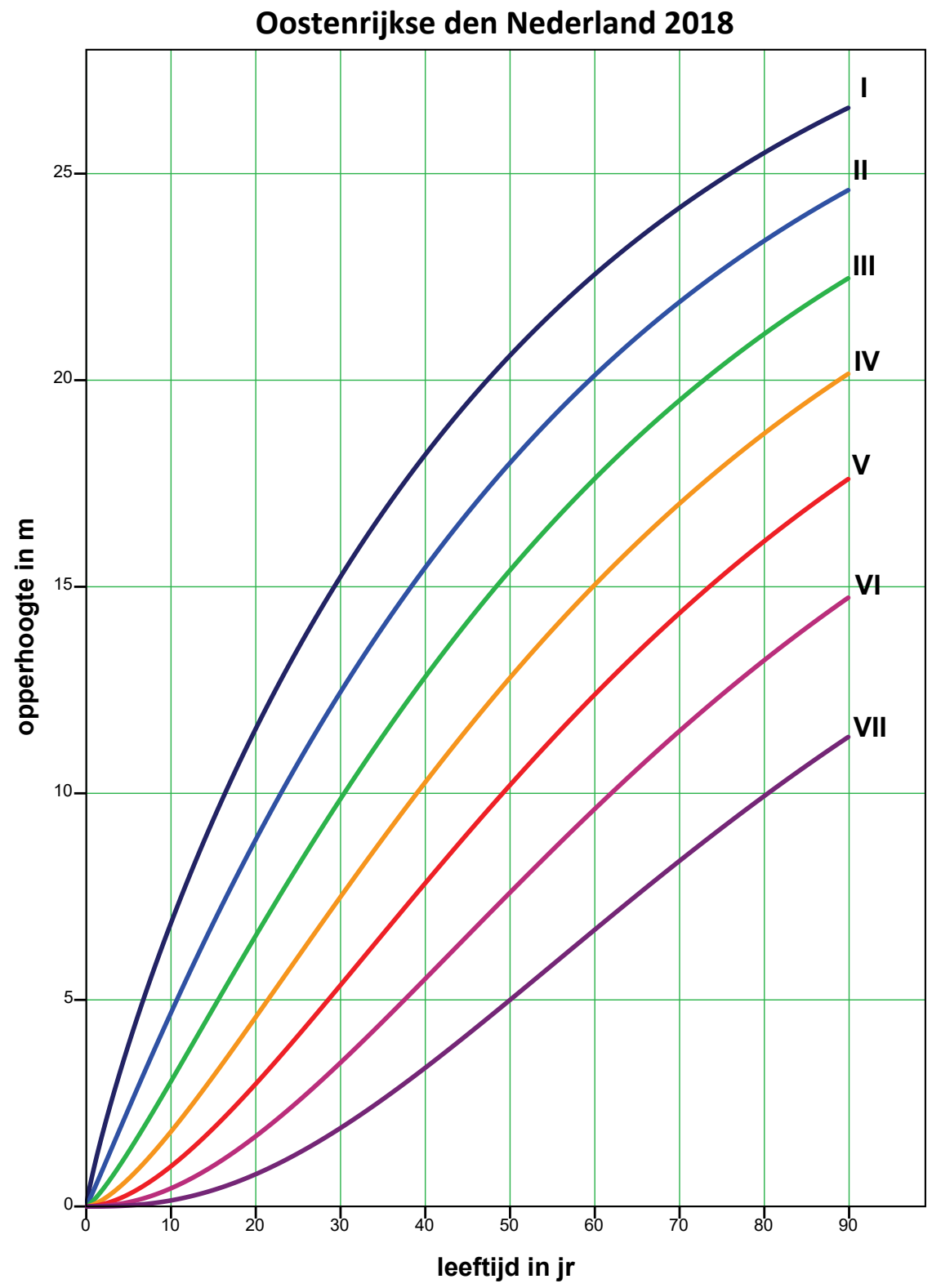




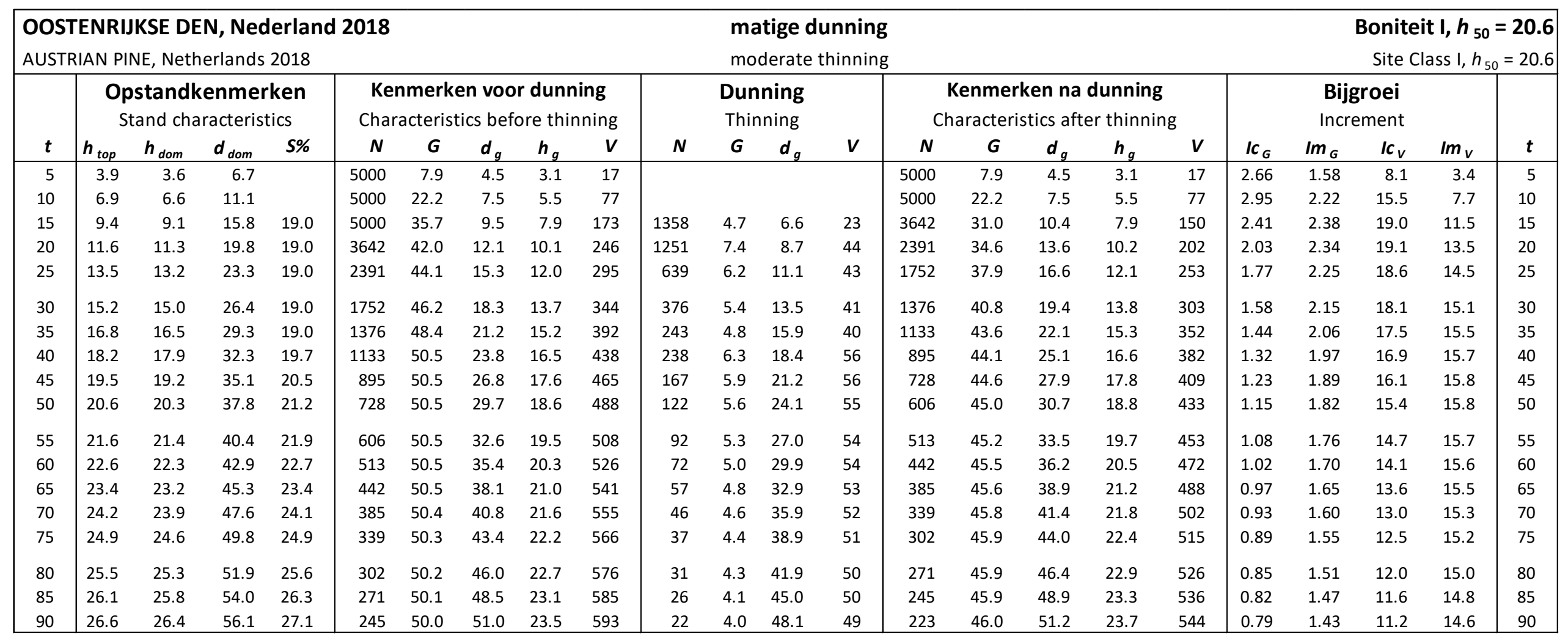




\begin{tabular}{|c|c|c|c|c|c|c|c|c|c|c|c|c|c|c|c|c|c|c|c|c|c|c|c|}
\hline OOS & 'ENRI & KSE D & $\mathrm{N}, \mathrm{Ne}$ & erlan & 2018 & & & & & & $\mathrm{~m}$ & tige & nni & & & & & & & & onite & $\mathrm{II}, h_{5}$ & 18.0 \\
\hline AUST & IAN P & $\mathrm{E}$, Net & erlands & 2018 & & & & & & & & derate & inn & & & & & & & & Site C & ss II, $h$ & $=18.0$ \\
\hline & & $\begin{array}{l}\text { tand } \\
\text { and ch }\end{array}$ & $\begin{array}{l}\text { enme } \\
\text { racteris }\end{array}$ & $\begin{array}{l}\text { ken } \\
\text { ics }\end{array}$ & $\begin{array}{r}\text { Ke } \\
\text { Char }\end{array}$ & acteris & $\begin{array}{l}\text { n voc } \\
\text { cs bef }\end{array}$ & $\begin{array}{l}\text { dun } \\
\text { ore thi }\end{array}$ & $\begin{array}{l}\text { ng } \\
\text { ning }\end{array}$ & & $\begin{array}{l}\text { Dur } \\
\text { Thi }\end{array}$ & $\begin{array}{l}\text { ning } \\
\text { ning }\end{array}$ & & & $\begin{array}{l}\text { enme } \\
\text { racter }\end{array}$ & $\begin{array}{l}\text { en na } \\
\text { tics aft }\end{array}$ & $\begin{array}{l}\text { Iunnin } \\
\text { r thinn }\end{array}$ & & & $\begin{array}{l}\text { Bij } \\
\text { Incr }\end{array}$ & $\begin{array}{c}\text { oei } \\
\text { nent }\end{array}$ & & \\
\hline$t$ & $h_{\text {top }}$ & $\boldsymbol{h}_{d o m}$ & $\boldsymbol{d}_{\text {dom }}$ & $S \%$ & $N$ & $G$ & $d_{g}$ & $h_{g}$ & $v$ & $N$ & $G$ & $d_{g}$ & $v$ & $N$ & $G$ & $d_{g}$ & $\boldsymbol{h}_{g}$ & $v$ & $I c_{G}$ & $I m_{G}$ & $I c_{v}$ & $I m_{V}$ & $t$ \\
\hline 5 & 2.4 & 2.1 & 3.6 & & 5000 & 2.4 & 2.4 & 1.9 & 3 & & & & & 5000 & 2.4 & 2.4 & $\begin{array}{c}9 \\
1.9\end{array}$ & 3 & 1.43 & 0.47 & 2.6 & 0.7 & 5 \\
\hline 10 & 4.7 & 4.4 & 8.0 & & 5000 & 11.5 & 5.4 & 3.8 & 29 & & & & & 5000 & 11.5 & 5.4 & 3.8 & 29 & 2.10 & 1.15 & 7.6 & 2.9 & 10 \\
\hline 15 & 6.9 & 6.6 & 11.3 & & 5000 & 22.7 & 7.6 & 5.5 & 78 & & & & & 5000 & 22.7 & 7.6 & 5.5 & 78 & 2.39 & 1.52 & 12.5 & 5.2 & 15 \\
\hline 20 & 8.9 & 8.6 & 14.8 & 19.0 & 5000 & 34.3 & 9.3 & 7.4 & 158 & 945 & 3.2 & 6.6 & 15 & 4055 & 31.1 & 9.9 & 7.5 & 143 & 2.13 & 1.72 & 15.8 & 7.9 & 20 \\
\hline 25 & 10.7 & 10.5 & 18.4 & 19.0 & 4055 & 41.0 & 11.3 & 9.3 & 224 & 1283 & 6.9 & 8.3 & 39 & 2772 & 34.1 & 12.5 & 9.4 & 186 & 1.85 & 1.77 & 16.3 & 9.6 & 25 \\
\hline 30 & 12.5 & 12.2 & 21.3 & 19.0 & 2772 & 42.8 & 14.0 & 11.0 & 267 & 710 & 6.0 & 10.4 & 38 & 2062 & 36.8 & 15.1 & 11.1 & 229 & 1.65 & 1.77 & 16.2 & 10.7 & 30 \\
\hline 35 & 14.0 & 13.8 & 24.1 & 19.0 & 2062 & 44.7 & 16.6 & 12.6 & 309 & 436 & 5.3 & 12.5 & 38 & 1626 & 39.4 & 17.6 & 12.7 & 271 & 1.50 & 1.74 & 16.0 & 11.5 & 35 \\
\hline 40 & 15.5 & 15.2 & 27.0 & 19.7 & 1626 & 46.5 & 19.1 & 14.0 & 350 & 386 & 6.6 & 14.8 & 51 & 1239 & 39.9 & 20.3 & 14.1 & 299 & 1.38 & 1.70 & 15.6 & 12.0 & 40 \\
\hline 45 & 16.8 & 16.5 & 29.8 & 20.5 & 1239 & 46.6 & 21.9 & 15.2 & 376 & 261 & 6.2 & 17.3 & 51 & 978 & 40.4 & 22.9 & 15.4 & 325 & 1.28 & 1.66 & 15.0 & 12.4 & 45 \\
\hline 50 & 18.0 & 17.7 & 32.5 & 21.2 & 978 & 46.6 & 24.6 & 16.4 & 399 & 185 & 5.8 & 20.0 & 51 & 793 & 40.8 & 25.6 & 16.5 & 348 & 1.19 & 1.62 & 14.5 & 12.6 & 50 \\
\hline 55 & 19.1 & 18.9 & 35.1 & 21.9 & 793 & 46.6 & 27.3 & 17.4 & 419 & 136 & 5.5 & 22.6 & 50 & 658 & 41.1 & 28.2 & 17.5 & 369 & 1.12 & 1.57 & 14.0 & 12.8 & 55 \\
\hline 60 & 20.1 & 19.9 & 37.5 & 22.7 & 658 & 46.5 & 30.0 & 18.3 & 437 & 102 & 5.2 & 25.4 & 50 & 555 & 41.4 & 30.8 & 18.5 & 388 & 1.06 & 1.53 & 13.5 & 12.8 & 60 \\
\hline 65 & 21.0 & 20.8 & 39.9 & 23.4 & 555 & 46.5 & 32.7 & 19.1 & 454 & 79 & 4.9 & 28.2 & 49 & 476 & 41.6 & 33.3 & 19.3 & 404 & 1.00 & 1.49 & 13.0 & 12.9 & 65 \\
\hline 70 & 21.9 & 21.6 & 42.2 & 24.1 & 476 & 46.5 & 35.2 & 19.8 & 468 & 62 & 4.7 & 31.0 & 49 & 414 & 41.8 & 35.8 & 20.0 & 420 & 0.96 & 1.46 & 12.6 & 12.9 & 70 \\
\hline 75 & 22.7 & 22.4 & 44.5 & 24.9 & 414 & 46.4 & 37.8 & 20.5 & 481 & 50 & 4.5 & 33.9 & 48 & 364 & 41.9 & 38.3 & 20.7 & 433 & 0.91 & 1.42 & 12.1 & 12.8 & 75 \\
\hline 80 & 23.4 & 23.1 & 46.6 & 25.6 & 364 & 46.4 & 40.3 & 21.1 & 493 & 41 & 4.3 & 36.8 & 47 & 323 & 42.0 & 40.7 & 21.3 & 446 & 0.88 & 1.39 & 11.7 & 12.8 & 80 \\
\hline 85 & 24.0 & 23.8 & 48.7 & 26.3 & 323 & 46.3 & 42.8 & 21.6 & 503 & 34 & 4.2 & 39.7 & 47 & 289 & 42.1 & 43.1 & 21.8 & 456 & 0.84 & 1.36 & 11.3 & 12.7 & 85 \\
\hline 90 & 24.6 & 24.4 & 50.8 & 27.1 & 289 & 46.3 & 45.2 & 22.1 & 512 & 28 & 4.0 & 42.6 & 46 & 260 & 42.2 & 45.4 & 22.3 & 466 & 0.81 & 1.33 & 10.9 & 12.6 & 90 \\
\hline
\end{tabular}




\begin{tabular}{|c|c|c|c|c|c|c|c|c|c|c|c|c|c|c|c|c|c|c|c|c|c|c|c|}
\hline \multicolumn{11}{|c|}{ OOSTENRIJKSE DEN, Nederland 2018} & \multicolumn{9}{|c|}{ matige dunning } & \multicolumn{4}{|c|}{ Boniteit III, $h_{50}=15.4$} \\
\hline \multicolumn{11}{|c|}{ AUSTRIAN PINE, Netherlands 2018} & \multicolumn{9}{|c|}{ moderate thinning } & \multicolumn{4}{|c|}{ Site Class III, $h_{50}=15.4$} \\
\hline \multirow[b]{2}{*}{$t$} & \multicolumn{4}{|c|}{$\begin{array}{l}\text { Opstandkenmerken } \\
\text { Stand characteristics }\end{array}$} & \multicolumn{5}{|c|}{$\begin{array}{l}\text { Kenmerken voor dunning } \\
\text { Characteristics before thinning }\end{array}$} & \multicolumn{4}{|c|}{$\begin{array}{l}\text { Dunning } \\
\text { Thinning }\end{array}$} & \multicolumn{5}{|c|}{$\begin{array}{c}\text { Kenmerken na dunning } \\
\text { Characteristics after thinning }\end{array}$} & \multicolumn{4}{|c|}{$\begin{array}{l}\text { Bijgroei } \\
\text { Increment }\end{array}$} & \multirow[b]{2}{*}{$t$} \\
\hline & $h_{\text {top }}$ & $\boldsymbol{h}_{\text {dom }}$ & $d_{d o m}$ & $S \%$ & $N$ & $\boldsymbol{G}$ & $d_{g}$ & $\boldsymbol{h}_{g}$ & $\boldsymbol{V}$ & $N$ & $\boldsymbol{G}$ & $d_{g}$ & $\boldsymbol{v}$ & $N$ & $\boldsymbol{G}$ & $d_{g}$ & $\boldsymbol{h}_{g}$ & $\boldsymbol{v}$ & $I c_{G}$ & $I m_{G}$ & $I c_{v}$ & $\operatorname{lm}_{V}$ & \\
\hline 5 & 1.3 & 1.1 & 0.3 & & 5000 & 0.0 & 0.2 & 1.1 & 0 & & & & & 5000 & 0.0 & 0.2 & 1.1 & 0 & 0.27 & 0.00 & 0.3 & 0.0 & 5 \\
\hline 10 & 3.0 & 2.7 & 5.1 & & 5000 & 4.7 & 3.5 & 2.4 & 8 & & & & & 5000 & 4.7 & 3.5 & 2.4 & 8 & 1.31 & 0.47 & 3.1 & 0.8 & 10 \\
\hline 15 & 4.8 & 4.5 & 8.3 & & 5000 & 12.4 & 5.6 & 3.8 & 32 & & & & & 5000 & 12.4 & 5.6 & 3.8 & 32 & 1.73 & 0.83 & 6.4 & 2.1 & 15 \\
\hline 20 & 6.6 & 6.3 & 11.0 & & 5000 & 21.7 & 7.4 & 5.2 & 72 & & & & & 5000 & 21.7 & 7.4 & 5.2 & 72 & 1.96 & 1.08 & 9.6 & 3.6 & 20 \\
\hline 25 & 8.3 & 8.0 & 13.7 & 19.0 & 5000 & 31.9 & 9.0 & 6.8 & 138 & 302 & 1.0 & 6.4 & 4 & 4698 & 30.9 & 9.2 & 6.9 & 133 & 1.96 & 1.28 & 13.4 & 5.5 & 25 \\
\hline 30 & 9.9 & 9.6 & 16.7 & 19.0 & 4698 & 40.1 & 10.4 & 8.4 & 203 & 1412 & 6.6 & 7.7 & 34 & 3286 & 33.5 & 11.4 & 8.5 & 169 & 1.74 & 1.37 & 14.2 & 6.9 & 30 \\
\hline 35 & \begin{tabular}{|l|}
11.4 \\
\end{tabular} & 11.1 & 19.2 & 19.0 & 3286 & 41.7 & 12.7 & 10.0 & 240 & 821 & 5.9 & 9.6 & 35 & 2465 & 35.8 & 13.6 & 10.1 & 205 & 1.57 & 1.41 & 14.2 & 8.0 & 35 \\
\hline 40 & $\mid 12.8$ & 12.6 & 22.0 & 19.7 & 2465 & 43.3 & 15.0 & 11.4 & 276 & 661 & 7.0 & 11.6 & 46 & 1804 & 36.4 & 16.0 & 11.5 & 231 & 1.44 & 1.42 & 14.1 & 8.7 & 40 \\
\hline 45 & \begin{tabular}{|l|}
14.2 \\
\end{tabular} & 13.9 & 24.7 & 20.5 & 1804 & 43.3 & 17.5 & 12.7 & 300 & 428 & 6.5 & 13.9 & 46 & 1376 & 36.8 & 18.5 & 12.9 & 254 & 1.33 & 1.42 & 13.8 & 9.3 & 45 \\
\hline 50 & 15.4 & 15.1 & 27.3 & 21.2 & 1376 & 43.2 & 20.0 & 14.0 & 323 & 292 & 6.0 & 16.2 & 46 & 1084 & 37.2 & 20.9 & 14.1 & 276 & 1.24 & 1.40 & 13.5 & 9.8 & 50 \\
\hline 55 & 16.6 & 16.3 & 29.9 & 21.9 & 1084 & 43.2 & 22.5 & 15.1 & 343 & 207 & 5.7 & 18.7 & 46 & 876 & 37.5 & 23.3 & 15.2 & 297 & 1.17 & 1.39 & 13.1 & 10.1 & 55 \\
\hline 60 & 17.6 & 17.4 & 32.3 & 22.7 & 876 & 43.2 & 25.0 & 16.1 & 361 & 152 & 5.4 & 21.2 & 46 & 724 & 37.8 & 25.8 & 16.2 & 315 & 1.10 & 1.36 & 12.7 & 10.3 & 60 \\
\hline 65 & 18.6 & 18.3 & 34.7 & 23.4 & 724 & 43.2 & 27.5 & 17.0 & 378 & 115 & 5.1 & 23.8 & 46 & 609 & 38.1 & 28.2 & 17.2 & 332 & 1.04 & 1.34 & 12.4 & 10.5 & 65 \\
\hline 70 & 19.5 & 19.3 & 37.0 & 24.1 & 609 & 43.1 & 30.0 & 17.8 & 393 & 89 & 4.9 & 26.4 & 45 & 521 & 38.3 & 30.6 & 18.0 & 347 & 0.99 & 1.32 & 12.0 & 10.6 & 70 \\
\hline 75 & 20.4 & 20.1 & 39.2 & 24.9 & 521 & 43.1 & 32.5 & 18.6 & 406 & 70 & 4.6 & 29.1 & 45 & 451 & 38.5 & 33.0 & 18.8 & 362 & 0.94 & 1.30 & 11.6 & 10.7 & 75 \\
\hline 80 & 21.1 & 20.9 & 41.4 & 25.6 & 451 & 43.1 & 34.9 & 19.3 & 419 & 56 & 4.4 & 31.8 & 44 & 395 & 38.6 & 35.3 & 19.5 & 374 & 0.90 & 1.27 & 11.3 & 10.7 & 80 \\
\hline 85 & 21.8 & 21.6 & 43.5 & 26.3 & 395 & 43.1 & 37.3 & 19.9 & 430 & 46 & 4.3 & 34.6 & 44 & 350 & 38.8 & 37.6 & 20.1 & 386 & 0.87 & 1.25 & 10.9 & 10.7 & 85 \\
\hline 90 & 22.5 & 22.2 & 45.5 & 27.1 & 350 & 43.0 & 39.6 & 20.5 & 440 & 38 & 4.1 & 37.3 & 43 & 312 & 38.9 & 39.9 & 20.7 & 397 & 0.83 & 1.23 & 10.6 & 10.7 & 90 \\
\hline
\end{tabular}




\begin{tabular}{|c|c|c|c|c|c|c|c|c|c|c|c|c|c|c|c|c|c|c|c|c|c|c|c|}
\hline \multicolumn{11}{|c|}{ OOSTENRIJKSE DEN, Nederland 2018} & \multicolumn{9}{|c|}{ matige dunning } & \multicolumn{4}{|c|}{ Boniteit IV, $h_{50}=12.8$} \\
\hline \multicolumn{11}{|c|}{ AUSTRIAN PINE, Netherlands 2018} & \multicolumn{9}{|c|}{ moderate thinning } & \multicolumn{4}{|c|}{ Site Class IV, $h_{50}=12.8$} \\
\hline \multirow[b]{2}{*}{$\boldsymbol{t}$} & \multicolumn{4}{|c|}{$\begin{array}{l}\text { Opstandkenmerken } \\
\text { Stand characteristics }\end{array}$} & \multicolumn{5}{|c|}{$\begin{array}{l}\text { Kenmerken voor dunning } \\
\text { Characteristics before thinning }\end{array}$} & \multicolumn{4}{|c|}{$\begin{array}{l}\text { Dunning } \\
\text { Thinning }\end{array}$} & \multicolumn{5}{|c|}{$\begin{array}{l}\text { Kenmerken na dunning } \\
\text { Characteristics after thinning }\end{array}$} & \multicolumn{4}{|c|}{$\begin{array}{c}\text { Bijgroei } \\
\text { Increment }\end{array}$} & \multirow[b]{2}{*}{$t$} \\
\hline & $\boldsymbol{h}_{\text {top }}$ & $\boldsymbol{h}_{\text {dom }}$ & $\boldsymbol{d}_{\text {dom }}$ & $S \%$ & $N$ & $\boldsymbol{G}$ & $d_{g}$ & $\boldsymbol{h}_{g}$ & $\boldsymbol{V}$ & $N$ & $\boldsymbol{G}$ & $d_{g}$ & $\boldsymbol{V}$ & $N$ & $\boldsymbol{G}$ & $d_{g}$ & $\boldsymbol{h}_{g}$ & $\boldsymbol{v}$ & $I c_{G}$ & $\operatorname{lm} m_{G}$ & $I c_{v}$ & $I m_{V}$ & \\
\hline 5 & 0.7 & 0.6 & & & 5000 & & & & 0 & & & & & 5000 & & & & 0 & & & 0.0 & 0.0 & 5 \\
\hline 10 & 1.8 & 1.5 & 2.3 & & 5000 & 0.9 & 1.5 & 1.5 & 1 & & & & & 5000 & 0.9 & 1.5 & 1.5 & 1 & 0.62 & 0.09 & 0.9 & 0.1 & 10 \\
\hline 15 & 3.2 & 2.9 & 5.5 & & 5000 & 5.4 & 3.7 & 2.5 & 10 & & & & & 5000 & 5.4 & 3.7 & 2.5 & 10 & 1.11 & 0.36 & 2.7 & 0.7 & 15 \\
\hline 20 & 4.6 & 4.3 & 8.1 & & 5000 & 11.8 & 5.5 & 3.7 & 29 & & & & & 5000 & 11.8 & 5.5 & 3.7 & 29 & 1.43 & 0.59 & 5.1 & 1.5 & 20 \\
\hline 25 & 6.0 & 5.8 & 10.5 & & 5000 & 19.6 & 7.1 & 4.8 & 61 & & & & & 5000 & 19.6 & 7.1 & 4.8 & 61 & 1.65 & 0.78 & 7.5 & 2.4 & 25 \\
\hline 30 & 7.5 & 7.2 & 12.6 & 20.3 & 5000 & 28.4 & 8.5 & 6.1 & 112 & & & & & 5000 & 28.4 & 8.5 & 6.1 & 112 & 1.85 & 0.95 & 13.9 & 3.7 & 30 \\
\hline 35 & 8.9 & 8.6 & 15.1 & 19.0 & 5000 & 37.2 & 9.7 & 7.5 & 172 & 969 & 4.0 & 7.3 & 19 & 4031 & 33.1 & 10.2 & 7.5 & 153 & 1.66 & 1.06 & 12.3 & 4.9 & 35 \\
\hline 40 & $\mid 10.3$ & 10.0 & 17.7 & 19.7 & 4031 & 41.0 & 11.4 & 8.9 & 215 & 1219 & 7.5 & 8.8 & 40 & 2812 & 33.5 & 12.3 & 8.9 & 175 & 1.52 & 1.13 & 12.5 & 5.9 & 40 \\
\hline 45 & 11.6 & 11.3 & 19.9 & 20.5 & 2812 & 40.8 & 13.6 & 10.2 & 237 & 752 & 6.9 & 10.8 & 41 & 2060 & 33.9 & 14.5 & 10.3 & 196 & 1.40 & 1.16 & 12.4 & 6.6 & 45 \\
\hline 50 & 12.8 & 12.5 & 22.3 & 21.2 & 2060 & 40.7 & 15.9 & 11.4 & 258 & 492 & 6.4 & 12.9 & 42 & 1569 & 34.3 & 16.7 & 11.5 & 216 & 1.30 & 1.18 & 12.3 & 7.2 & 50 \\
\hline 55 & 14.0 & 13.7 & 24.8 & 21.9 & 1569 & 40.6 & 18.1 & 12.6 & 277 & 337 & 6.0 & 15.1 & 42 & 1232 & 34.6 & 18.9 & 12.7 & 235 & 1.22 & 1.19 & 12.0 & 7.6 & 55 \\
\hline 60 & 15.0 & 14.8 & 27.2 & 22.7 & 1232 & 40.5 & 20.5 & 13.7 & 295 & 239 & 5.6 & 17.3 & 42 & 993 & 34.8 & 21.1 & 13.8 & 253 & 1.15 & 1.19 & 11.8 & 8.0 & 60 \\
\hline 65 & 16.1 & 15.8 & 29.5 & 23.4 & 993 & 40.4 & 22.8 & 14.7 & 311 & 175 & 5.3 & 19.7 & 42 & 817 & 35.1 & 23.4 & 14.8 & 269 & 1.09 & 1.18 & 11.5 & 8.3 & 65 \\
\hline 70 & $\mid 17.0$ & 16.8 & 31.7 & 24.1 & 817 & 40.4 & 25.1 & 15.6 & 326 & 132 & 5.1 & 22.1 & 42 & 685 & 35.3 & 25.6 & 15.7 & 284 & 1.03 & 1.17 & 11.3 & 8.5 & 70 \\
\hline 75 & 17.9 & 17.6 & 33.9 & 24.9 & 685 & 40.3 & 27.4 & 16.4 & 340 & 102 & 4.8 & 24.5 & 42 & 583 & 35.5 & 27.8 & 16.6 & 298 & 0.98 & 1.16 & 11.0 & 8.7 & 75 \\
\hline 80 & $\mid 18.7$ & 18.5 & 36.1 & 25.6 & 583 & 40.3 & 29.7 & 17.2 & 352 & 80 & 4.6 & 27.1 & 41 & 504 & 35.7 & 30.0 & 17.4 & 311 & 0.94 & 1.15 & 10.7 & 8.8 & 80 \\
\hline 85 & 19.5 & 19.2 & 38.2 & 26.3 & 504 & 40.3 & 31.9 & 17.9 & 364 & 64 & 4.4 & 29.6 & 41 & 440 & 35.9 & 32.2 & 18.1 & 323 & 0.90 & 1.14 & 10.4 & 8.9 & 85 \\
\hline 90 & 20.2 & 19.9 & 40.2 & 27.1 & 440 & 40.3 & 34.2 & 18.6 & 374 & 52 & 4.2 & 32.2 & 40 & 388 & 36.1 & 34.4 & 18.7 & 334 & 0.86 & 1.12 & 10.1 & 9.0 & 90 \\
\hline
\end{tabular}




\begin{tabular}{|c|c|c|c|c|c|c|c|c|c|c|c|c|c|c|c|c|c|c|c|c|c|c|c|}
\hline \multicolumn{11}{|c|}{ OOSTENRIJKSE DEN, Nederland 2018} & \multicolumn{9}{|c|}{ matige dunning } & \multicolumn{4}{|c|}{ Boniteit V, $h_{50}=10.2$} \\
\hline \multicolumn{11}{|c|}{ AUSTRIAN PINE, Netherlands 2018} & \multicolumn{9}{|c|}{ moderate thinning } & \multicolumn{4}{|c|}{ Site Class V, $h_{50}=10.2$} \\
\hline \multirow[b]{2}{*}{$\boldsymbol{t}$} & \multicolumn{4}{|c|}{$\begin{array}{l}\text { Opstandkenmerken } \\
\text { Stand characteristics }\end{array}$} & \multicolumn{5}{|c|}{$\begin{array}{l}\text { Kenmerken voor dunning } \\
\text { Characteristics before thinning }\end{array}$} & \multicolumn{4}{|c|}{$\begin{array}{l}\text { Dunning } \\
\text { Thinning }\end{array}$} & \multicolumn{5}{|c|}{$\begin{array}{l}\text { Kenmerken na dunning } \\
\text { Characteristics after thinning }\end{array}$} & \multicolumn{4}{|c|}{$\begin{array}{c}\text { Bijgroei } \\
\text { Increment }\end{array}$} & \multirow[b]{2}{*}{$\boldsymbol{t}$} \\
\hline & $\boldsymbol{h}_{\text {top }}$ & $\boldsymbol{h}_{\text {dom }}$ & $d_{d o m}$ & $S \%$ & $N$ & $\boldsymbol{G}$ & $d_{g}$ & $\boldsymbol{h}_{g}$ & $\boldsymbol{V}$ & $N$ & $\boldsymbol{G}$ & $d_{g}$ & $\boldsymbol{V}$ & $N$ & $\boldsymbol{G}$ & $d_{g}$ & $\boldsymbol{h}_{g}$ & $\boldsymbol{v}$ & $I c_{G}$ & $\operatorname{lm} m_{G}$ & $I c_{v}$ & $I m_{V}$ & \\
\hline 5 & 0.3 & 0.3 & & & 5000 & & & & 0 & & & & & 5000 & & & & 0 & & & 0.0 & 0.0 & 5 \\
\hline 10 & 1.0 & 0.8 & & & 5000 & & & & 0 & & & & & 5000 & & & & 0 & & & 0.0 & 0.0 & 10 \\
\hline 15 & 1.9 & 1.6 & 2.6 & & 5000 & 1.2 & 1.7 & 1.5 & 1 & & & & & 5000 & 1.2 & 1.7 & 1.5 & 1 & 0.55 & 0.08 & 0.8 & 0.1 & 15 \\
\hline 20 & 3.0 & 2.7 & 5.2 & & 5000 & 4.9 & 3.5 & 2.4 & 8 & & & & & 5000 & 4.9 & 3.5 & 2.4 & 8 & 0.91 & 0.24 & 2.1 & 0.4 & 20 \\
\hline 25 & 4.1 & 3.9 & 7.5 & & 5000 & 10.1 & 5.1 & 3.3 & 23 & & & & & 5000 & 10.1 & 5.1 & 3.3 & 23 & 1.18 & 0.40 & 3.8 & 0.9 & 25 \\
\hline 30 & 5.4 & 5.1 & 9.6 & & 5000 & 16.5 & 6.5 & 4.3 & 46 & & & & & 5000 & 16.5 & 6.5 & 4.3 & 46 & 1.38 & 0.55 & 5.6 & 1.5 & 30 \\
\hline 35 & 6.6 & 6.3 & 11.6 & & 5000 & 23.8 & 7.8 & 5.3 & 79 & & & & & 5000 & 23.8 & 7.8 & 5.3 & 79 & 1.53 & 0.68 & 7.6 & 2.3 & 35 \\
\hline 40 & 7.8 & 7.6 & 13.4 & 19.7 & 5000 & 32.0 & 9.0 & 6.4 & 132 & 158 & 0.6 & 6.9 & 2 & 4842 & 31.4 & 9.1 & 6.5 & 129 & 1.62 & 0.80 & 10.3 & 3.3 & 40 \\
\hline 45 & 9.0 & 8.8 & 15.6 & 20.5 & 4842 & 39.2 & 10.1 & 7.6 & 183 & 1462 & 7.5 & 8.1 & 36 & 3380 & 31.7 & 10.9 & 7.7 & 147 & 1.48 & 0.88 & 10.9 & 4.1 & 45 \\
\hline 50 & 10.2 & 9.9 & 18.0 & 21.2 & 3380 & 38.8 & 12.1 & 8.8 & 202 & 910 & 6.9 & 9.8 & 37 & 2470 & 31.9 & 12.8 & 8.9 & 165 & 1.38 & 0.94 & 10.9 & 4.8 & 50 \\
\hline 55 & $\mid 11.3$ & 11.1 & 19.8 & 21.9 & 2470 & 38.5 & 14.1 & 10.0 & 219 & 597 & 6.4 & 11.7 & 37 & 1873 & 32.1 & 14.8 & 10.1 & 182 & 1.28 & 0.97 & 10.8 & 5.3 & 55 \\
\hline 60 & 12.4 & 12.1 & 22.0 & 22.7 & 1873 & 38.3 & 16.1 & 11.1 & 235 & 409 & 6.0 & 13.7 & 38 & 1464 & 32.3 & 16.8 & 11.2 & 197 & 1.21 & 1.00 & 10.7 & 5.8 & 60 \\
\hline 65 & 13.4 & 13.1 & 24.2 & 23.4 & 1464 & 38.1 & 18.2 & 12.1 & 251 & 290 & 5.7 & 15.8 & 38 & 1174 & 32.5 & 18.8 & 12.2 & 212 & 1.14 & 1.01 & 10.6 & 6.2 & 65 \\
\hline 70 & $\mid$\begin{tabular}{|l|}
14.4 \\
\end{tabular} & 14.1 & 26.3 & 24.1 & 1174 & 38.0 & 20.3 & 13.1 & 265 & 212 & 5.3 & 17.9 & 38 & 962 & 32.7 & 20.8 & 13.2 & 227 & 1.08 & 1.02 & 10.4 & 6.5 & 70 \\
\hline 75 & 15.3 & 15.0 & 28.5 & 24.9 & 962 & 37.9 & 22.4 & 14.0 & 278 & 159 & 5.1 & 20.1 & 38 & 802 & 32.9 & 22.8 & 14.1 & 240 & 1.03 & 1.02 & 10.2 & 6.7 & 75 \\
\hline 80 & $\mid 16.1$ & 15.8 & 30.5 & 25.6 & 802 & 37.9 & 24.5 & 14.8 & 290 & 122 & 4.8 & 22.4 & 38 & 680 & 33.1 & 24.9 & 14.9 & 253 & 0.98 & 1.02 & 10.0 & 6.9 & 80 \\
\hline 85 & 16.9 & 16.6 & 32.6 & 26.3 & 680 & 37.8 & 26.6 & 15.6 & 302 & 96 & 4.6 & 24.7 & 38 & 584 & 33.3 & 26.9 & 15.7 & 264 & 0.93 & 1.01 & 9.8 & 7.1 & 85 \\
\hline 90 & 17.6 & 17.4 & 34.5 & 27.1 & 584 & 37.8 & 28.7 & 16.3 & 313 & 76 & 4.4 & 27.1 & 37 & 508 & 33.4 & 29.0 & 16.4 & 275 & 0.90 & 1.01 & 9.5 & 7.3 & 90 \\
\hline
\end{tabular}




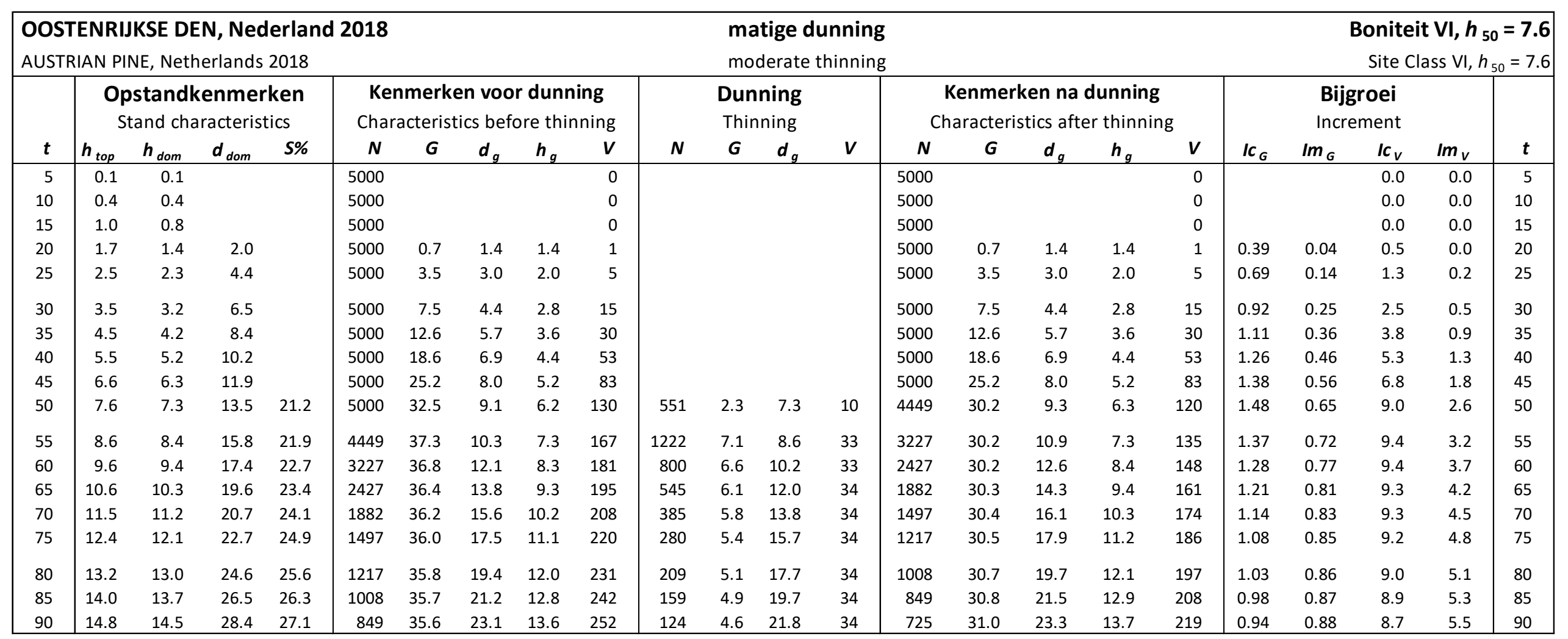




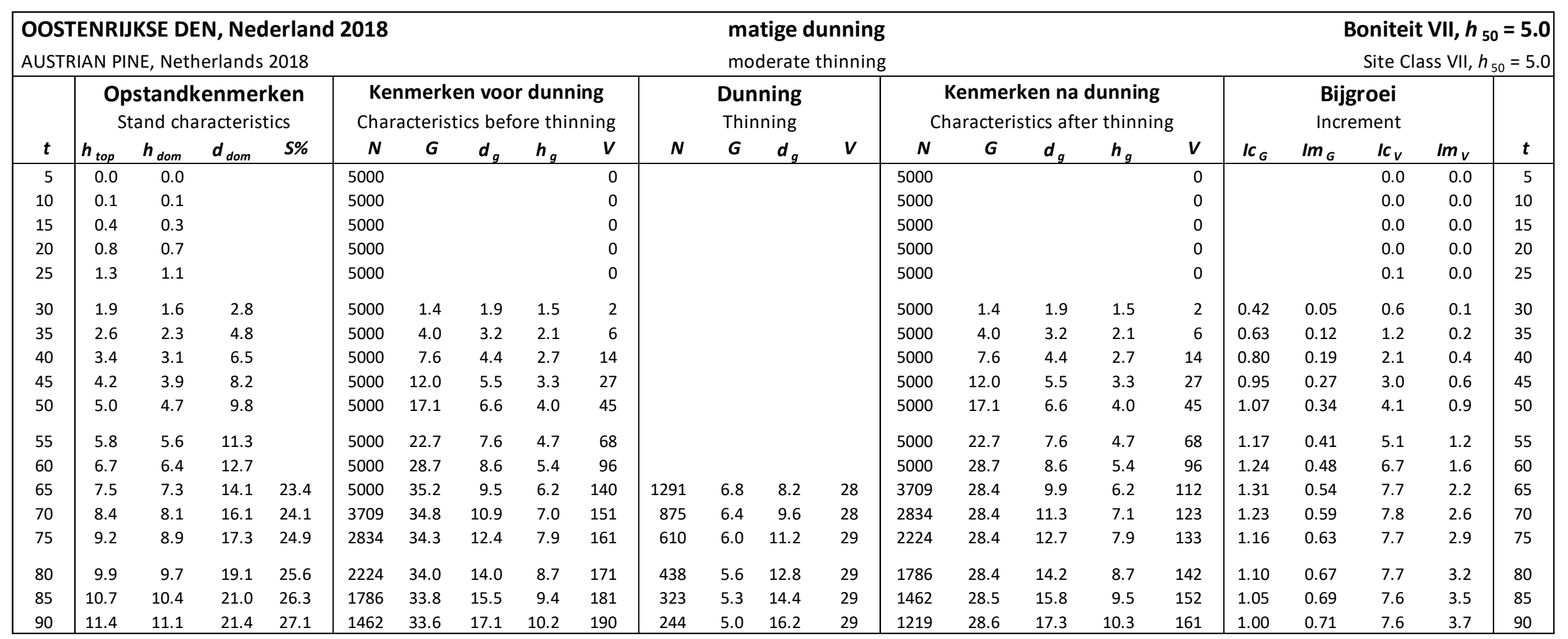




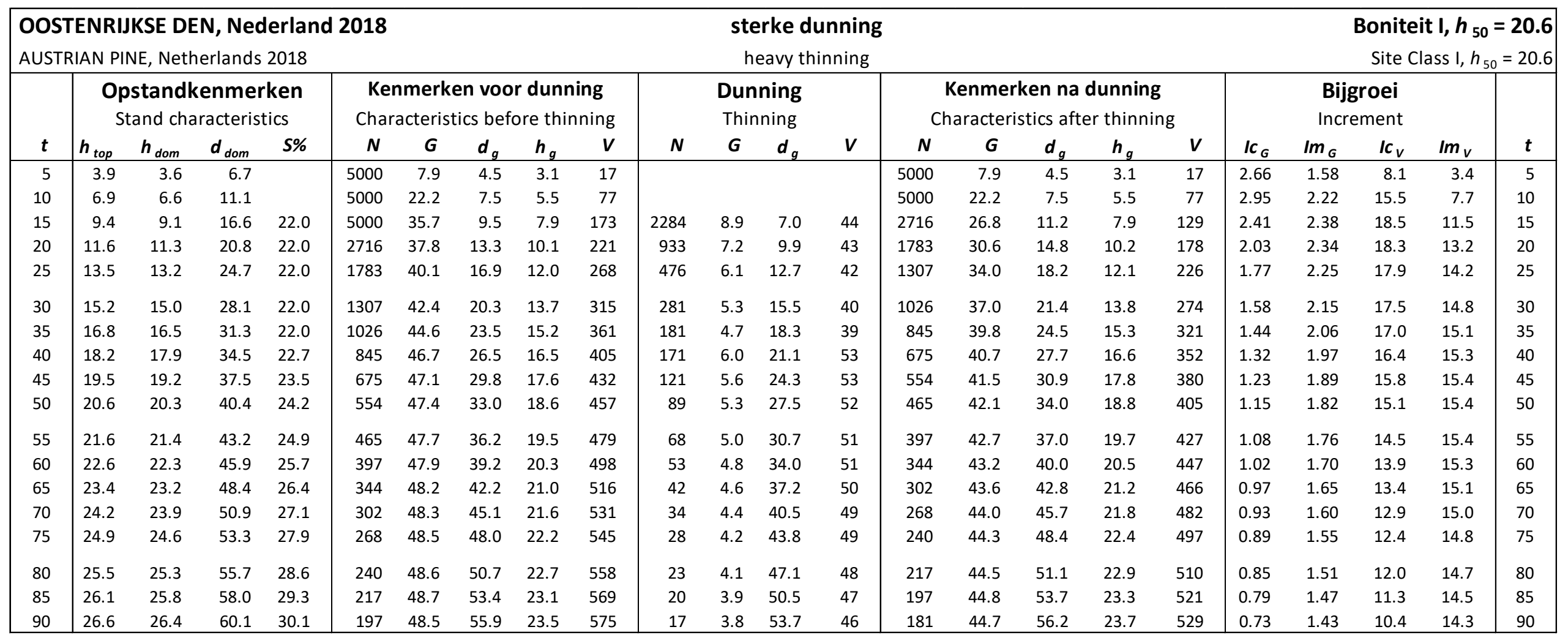




\begin{tabular}{|c|c|c|c|c|c|c|c|c|c|c|c|c|c|c|c|c|c|c|c|c|c|c|c|}
\hline OOS & -ENRI & KSE D & $\mathrm{V}, \mathrm{Ne}$ & erlan & 2018 & & & & & & & rke & nnin & & & & & & & & onite & II, $h_{5}$ & 18.0 \\
\hline AUST & IAN P & $\mathrm{E}$, Net & erlands & 2018 & & & & & & & & eavy $t$ & ining & & & & & & & & Site C & ss II, $h$ & $=18.0$ \\
\hline & & $\begin{array}{l}\text { tand } \\
\text { and ch }\end{array}$ & $\begin{array}{l}\text { enme } \\
\text { acteris }\end{array}$ & $\begin{array}{l}\text { ken } \\
\text { ics }\end{array}$ & $\begin{array}{r}\text { Ke } \\
\text { Char }\end{array}$ & acteris & $\begin{array}{l}\text { n voc } \\
\text { cs bef }\end{array}$ & $\begin{array}{l}\text { dun } \\
\text { ore thi }\end{array}$ & $\begin{array}{l}\text { ng } \\
\text { ning }\end{array}$ & & $\begin{array}{l}\text { Dur } \\
\text { Thi }\end{array}$ & $\begin{array}{l}\text { ning } \\
\text { ning }\end{array}$ & & & $\begin{array}{l}\text { enme } \\
\text { racter }\end{array}$ & $\begin{array}{l}\text { en na } \\
\text { tics aft }\end{array}$ & $\begin{array}{l}\text { Iunnin } \\
\text { r thinn }\end{array}$ & & & $\begin{array}{l}\text { Bij } \\
\text { Incr }\end{array}$ & $\begin{array}{l}\text { oei } \\
\text { nent }\end{array}$ & & \\
\hline$t$ & $h_{\text {top }}$ & $\boldsymbol{h}_{\text {dom }}$ & $\boldsymbol{d}_{\text {dom }}$ & S\% & $N$ & $\boldsymbol{G}$ & $d_{g}$ & $\boldsymbol{h}_{g}$ & $v$ & $N$ & $\boldsymbol{G}$ & $d_{g}$ & $v$ & $N$ & $G$ & $d_{g}$ & $\boldsymbol{h}_{g}$ & $v$ & $I c_{G}$ & $I m_{G}$ & $I c_{v}$ & $I m_{V}$ & $t$ \\
\hline 5 & 2.4 & 2.1 & 3.6 & & 5000 & 2.4 & 2.4 & 1.9 & 3 & & & & & 5000 & 2.4 & 2.4 & 1.9 & 3 & 1.43 & 0.47 & 2.6 & 0.7 & 5 \\
\hline 10 & 4.7 & 4.4 & 8.0 & & 5000 & 11.5 & 5.4 & 3.8 & 29 & & & & & 5000 & 11.5 & 5.4 & 3.8 & 29 & 2.10 & 1.15 & 7.6 & 2.9 & 10 \\
\hline 15 & 6.9 & 6.6 & 11.3 & & 5000 & 22.7 & 7.6 & 5.5 & 78 & & & & & 5000 & 22.7 & 7.6 & 5.5 & 78 & 2.39 & 1.52 & 12.5 & 5.2 & 15 \\
\hline 20 & 8.9 & 8.6 & 15.7 & 22.0 & 5000 & 34.3 & 9.3 & 7.4 & 158 & 1976 & 7.6 & 7.0 & 36 & 3024 & 26.7 & 10.6 & 7.5 & 123 & 2.13 & 1.72 & 15.4 & 7.9 & 20 \\
\hline 25 & 10.7 & 10.5 & 19.5 & 22.0 & 3024 & 36.6 & 12.4 & 9.3 & 200 & 957 & 6.6 & 9.4 & 37 & 2068 & 30.0 & 13.6 & 9.4 & 163 & 1.85 & 1.77 & 15.6 & 9.4 & 25 \\
\hline 30 & 12.5 & 12.2 & 22.5 & 22.0 & 2068 & 38.7 & 15.4 & 11.0 & 241 & 529 & 5.8 & 11.8 & 37 & 1538 & 32.9 & 16.5 & 11.1 & 204 & 1.65 & 1.77 & 15.6 & 10.4 & 30 \\
\hline 35 & 14.0 & 13.8 & 25.6 & 22.0 & 1538 & 40.8 & 18.4 & 12.6 & 281 & 326 & 5.2 & 14.3 & 37 & 1213 & 35.5 & 19.3 & 12.7 & 244 & 1.50 & 1.74 & 15.4 & 11.2 & 35 \\
\hline 40 & 15.5 & 15.2 & 28.7 & 22.7 & 1213 & 42.7 & 21.2 & 14.0 & 321 & 279 & 6.3 & 16.9 & 48 & 934 & 36.5 & 22.3 & 14.1 & 273 & 1.38 & 1.70 & 15.1 & 11.7 & 40 \\
\hline 45 & $\mid 16.8$ & 16.5 & 31.7 & 23.5 & 934 & 43.1 & 24.2 & 15.2 & 347 & 190 & 5.8 & 19.8 & 48 & 744 & 37.3 & 25.2 & 15.4 & 299 & 1.28 & 1.66 & 14.7 & 12.0 & 45 \\
\hline 50 & 18.0 & 17.7 & 34.6 & 24.2 & 744 & 43.4 & 27.3 & 16.4 & 371 & 135 & 5.5 & 22.7 & 48 & 609 & 37.9 & 28.2 & 16.5 & 323 & 1.19 & 1.62 & 14.2 & 12.3 & 50 \\
\hline 55 & 19.1 & 18.9 & 37.4 & 24.9 & 609 & 43.7 & 30.2 & 17.4 & 393 & 100 & 5.2 & 25.7 & 48 & 509 & 38.5 & 31.1 & 17.5 & 345 & 1.12 & 1.57 & 13.8 & 12.4 & 55 \\
\hline 60 & 20.1 & 19.9 & 40.1 & 25.7 & 509 & 44.0 & 33.2 & 18.3 & 413 & 76 & 4.9 & 28.7 & 47 & 433 & 39.1 & 33.9 & 18.5 & 366 & 1.06 & 1.53 & 13.3 & 12.5 & 60 \\
\hline 65 & 21.0 & 20.8 & 42.6 & 26.4 & 433 & 44.2 & 36.1 & 19.1 & 431 & 59 & 4.7 & 31.8 & 47 & 374 & 39.5 & 36.7 & 19.3 & 384 & 1.00 & 1.49 & 12.9 & 12.6 & 65 \\
\hline 70 & 21.9 & 21.6 & 45.1 & 27.1 & 374 & 44.4 & 38.9 & 19.8 & 447 & 47 & 4.5 & 34.9 & 46 & 327 & 39.9 & 39.4 & 20.0 & 401 & 0.96 & 1.46 & 12.4 & 12.6 & 70 \\
\hline 75 & 22.7 & 22.4 & 47.5 & 27.9 & 327 & 44.6 & 41.7 & 20.5 & 462 & 38 & 4.3 & 38.1 & 46 & 290 & 40.3 & 42.1 & 20.7 & 416 & 0.91 & 1.42 & 12.0 & 12.5 & 75 \\
\hline 80 & 23.4 & 23.1 & 49.9 & 28.6 & 290 & 44.8 & 44.4 & 21.1 & 475 & 31 & 4.1 & 41.2 & 45 & 258 & 40.6 & 44.7 & 21.3 & 430 & 0.88 & 1.39 & 11.6 & 12.5 & 80 \\
\hline 85 & 24.0 & 23.8 & 52.1 & 29.3 & 258 & 44.9 & 47.0 & 21.6 & 487 & 26 & 4.0 & 44.4 & 44 & 233 & 40.9 & 47.3 & 21.8 & 443 & 0.82 & 1.36 & 11.0 & 12.4 & 85 \\
\hline 90 & 24.6 & 24.4 & 54.3 & 30.1 & 233 & 44.8 & 49.5 & 22.1 & 495 & 22 & 3.8 & 47.5 & 44 & 211 & 40.9 & 49.7 & 22.3 & 452 & 0.75 & 1.33 & 10.2 & 12.3 & 90 \\
\hline
\end{tabular}




\begin{tabular}{|c|c|c|c|c|c|c|c|c|c|c|c|c|c|c|c|c|c|c|c|c|c|c|c|}
\hline OOS & -ENRI & KSE D & V, Nec & erlan & 2018 & & & & & & & rke & nnin & & & & & & & & nitei & $\mathrm{III}, h_{5}$ & 15.4 \\
\hline AUST & IAN P & $\mathrm{E}$, Net & erlands & 2018 & & & & & & & & eavy $t$ & ining & & & & & & & & Site C & s III, $h$ & \\
\hline & & $\begin{array}{l}\text { tand } \\
\text { and ch }\end{array}$ & $\begin{array}{l}\text { enme } \\
\text { acteris }\end{array}$ & $\begin{array}{l}\text { ken } \\
\text { ics }\end{array}$ & $\begin{array}{r}\text { Ke } \\
\text { Char }\end{array}$ & acteris & $\begin{array}{l}\text { n voc } \\
\text { cs bef }\end{array}$ & $\begin{array}{l}\text { dun } \\
\text { ore thi }\end{array}$ & $\begin{array}{l}\text { ng } \\
\text { ning }\end{array}$ & & $\begin{array}{l}\text { Dur } \\
\text { Thi }\end{array}$ & $\begin{array}{l}\text { ning } \\
\text { ning }\end{array}$ & & & $\begin{array}{l}\text { enme } \\
\text { racter }\end{array}$ & $\begin{array}{l}\text { en na } \\
\text { tics aft }\end{array}$ & $\begin{array}{l}\text { Iunnin } \\
\text { r thinn }\end{array}$ & & & $\begin{array}{l}\text { Bij } \\
\text { Incr }\end{array}$ & $\begin{array}{l}\text { oei } \\
\text { nent }\end{array}$ & & \\
\hline$t$ & $h_{\text {top }}$ & $\boldsymbol{h}_{\text {dom }}$ & $\boldsymbol{d}_{\text {dom }}$ & S\% & $N$ & $\boldsymbol{G}$ & $d_{g}$ & $\boldsymbol{h}_{g}$ & $v$ & $N$ & $\boldsymbol{G}$ & $d_{g}$ & $v$ & $N$ & $G$ & $d_{g}$ & $h_{g}$ & $v$ & $I c_{G}$ & $I m_{G}$ & $I c_{v}$ & $\operatorname{lm}{ }_{V}$ & $t$ \\
\hline 5 & 1.3 & 1.1 & 0.3 & & 5000 & 0.0 & 0.2 & 1.1 & 0 & & & & & 5000 & 0.0 & 0.2 & 1.1 & 0 & 0.27 & 0.00 & 0.3 & 0.0 & 5 \\
\hline 10 & 3.0 & 2.7 & 5.1 & & 5000 & 4.7 & 3.5 & 2.4 & 8 & & & & & 5000 & 4.7 & 3.5 & 2.4 & 8 & 1.31 & 0.47 & 3.1 & 0.8 & 10 \\
\hline 15 & 4.8 & 4.5 & 8.3 & & 5000 & 12.4 & 5.6 & 3.8 & 32 & & & & & 5000 & 12.4 & 5.6 & 3.8 & 32 & 1.73 & 0.83 & 6.4 & 2.1 & 15 \\
\hline 20 & 6.6 & 6.3 & 11.0 & & 5000 & 21.7 & 7.4 & 5.2 & 72 & & & & & 5000 & 21.7 & 7.4 & 5.2 & 72 & 1.96 & 1.08 & 9.6 & 3.6 & 20 \\
\hline 25 & 8.3 & 8.0 & 14.5 & 22.0 & 5000 & 31.9 & 9.0 & 6.8 & 138 & 1496 & 5.4 & 6.8 & 24 & 3504 & 26.4 & 9.8 & 6.9 & 114 & 1.96 & 1.28 & 13.0 & 5.5 & 25 \\
\hline 30 & 9.9 & 9.6 & 17.7 & 22.0 & 3504 & 35.6 & 11.4 & 8.4 & 180 & 1053 & 6.3 & 8.8 & 33 & 2451 & 29.3 & 12.3 & 8.5 & 147 & 1.74 & 1.37 & 13.5 & 6.8 & 30 \\
\hline 35 & $\mid 11.4$ & 11.1 & 20.3 & 22.0 & 2451 & 37.6 & 14.0 & 10.0 & 215 & 612 & 5.7 & 10.9 & 34 & 1839 & 31.8 & 14.9 & 10.1 & 182 & 1.57 & 1.41 & 13.7 & 7.8 & 35 \\
\hline 40 & 12.8 & 12.6 & 23.3 & 22.7 & 1839 & 39.4 & 16.5 & 11.4 & 250 & 479 & 6.6 & 13.2 & 43 & 1359 & 32.8 & 17.5 & 11.5 & 208 & 1.44 & 1.42 & 13.6 & 8.5 & 40 \\
\hline 45 & \begin{tabular}{|l|}
14.2 \\
\end{tabular} & 13.9 & 26.2 & 23.5 & 1359 & 39.7 & 19.3 & 12.7 & 275 & 313 & 6.1 & 15.8 & 43 & 1046 & 33.6 & 20.2 & 12.9 & 232 & 1.33 & 1.42 & 13.4 & 9.1 & 45 \\
\hline 50 & 15.4 & 15.1 & 29.0 & 24.2 & 1046 & 40.0 & 22.1 & 14.0 & 298 & 215 & 5.7 & 18.4 & 44 & 832 & 34.3 & 22.9 & 14.1 & 255 & 1.24 & 1.40 & 13.1 & 9.5 & 50 \\
\hline 55 & 16.6 & 16.3 & 31.8 & 24.9 & 832 & 40.3 & 24.9 & 15.1 & 319 & 153 & 5.4 & 21.1 & 44 & 678 & 35.0 & 25.6 & 15.2 & 276 & 1.17 & 1.39 & 12.8 & 9.8 & 55 \\
\hline 60 & \begin{tabular}{|l}
17.6 \\
\end{tabular} & 17.4 & 34.4 & 25.7 & 678 & 40.6 & 27.6 & 16.1 & 339 & 113 & 5.1 & 23.9 & 44 & 565 & 35.5 & 28.3 & 16.2 & 295 & 1.10 & 1.36 & 12.5 & 10.0 & 60 \\
\hline 65 & 18.6 & 18.3 & 37.0 & 26.4 & 565 & 40.9 & 30.4 & 17.0 & 357 & 86 & 4.9 & 26.8 & 43 & 479 & 36.0 & 30.9 & 17.2 & 314 & 1.04 & 1.34 & 12.2 & 10.2 & 65 \\
\hline 70 & 19.5 & 19.3 & 39.4 & 27.1 & 479 & 41.1 & 33.1 & 17.8 & 374 & 67 & 4.6 & 29.7 & 43 & 412 & 36.5 & 33.6 & 18.0 & 331 & 0.99 & 1.32 & 11.8 & 10.3 & 70 \\
\hline 75 & 20.4 & 20.1 & 41.8 & 27.9 & 412 & 41.3 & 35.7 & 18.6 & 389 & 53 & 4.4 & 32.7 & 43 & 359 & 36.9 & 36.1 & 18.8 & 346 & 0.94 & 1.30 & 11.5 & 10.4 & 75 \\
\hline 80 & 21.1 & 20.9 & 44.1 & 28.6 & 359 & 41.5 & 38.3 & 19.3 & 403 & 43 & 4.3 & 35.6 & 42 & 317 & 37.2 & 38.7 & 19.5 & 360 & 0.90 & 1.27 & 11.2 & 10.5 & 80 \\
\hline 85 & 21.8 & 21.6 & 46.4 & 29.3 & 317 & 41.7 & 40.9 & 19.9 & 415 & 35 & 4.1 & 38.7 & 42 & 282 & 37.6 & 41.2 & 20.1 & 374 & 0.84 & 1.25 & 10.6 & 10.5 & 85 \\
\hline 90 & 22.5 & 22.2 & 48.5 & 30.1 & 282 & 41.6 & 43.3 & 20.5 & 424 & 29 & 3.9 & 41.6 & 41 & 253 & 37.6 & 43.5 & 20.7 & 383 & 0.77 & 1.22 & 9.8 & 10.5 & 90 \\
\hline
\end{tabular}




\begin{tabular}{|c|c|c|c|c|c|c|c|c|c|c|c|c|c|c|c|c|c|c|c|c|c|c|c|}
\hline OOS & ENR & KSE D & $\mathrm{V}, \mathrm{Ne}$ & erlan & 2018 & & & & & & & rke & nnin & & & & & & & & nitei & $\mathrm{V}, h_{5}$ & 12.8 \\
\hline AUST & IAN P & $\mathrm{E}$, Net & erlands & 2018 & & & & & & & & eavy $t$ & ining & & & & & & & & Site $\mathrm{Cl}$ & s IV, $h$ & \\
\hline & & $\begin{array}{l}\text { tand } \\
\text { and ch }\end{array}$ & $\begin{array}{l}\text { enme } \\
\text { acteris }\end{array}$ & $\begin{array}{l}\text { ken } \\
\text { ics }\end{array}$ & $\begin{array}{r}\text { Ke } \\
\text { Char }\end{array}$ & acteris & $\begin{array}{l}\text { n voc } \\
\text { cs bef }\end{array}$ & $\begin{array}{l}\text { dunr } \\
\text { ore thi }\end{array}$ & $\begin{array}{l}\text { ng } \\
\text { ning }\end{array}$ & & $\begin{array}{l}\text { Dur } \\
\text { Thi }\end{array}$ & $\begin{array}{l}\text { ning } \\
\text { ning }\end{array}$ & & & $\begin{array}{l}\text { enme } \\
\text { racter }\end{array}$ & $\begin{array}{l}\text { en na } \\
\text { tics aft }\end{array}$ & $\begin{array}{l}\text { Iunnin } \\
\text { r thinn }\end{array}$ & & & $\begin{array}{l}\text { Bij } \\
\text { Incr }\end{array}$ & $\begin{array}{l}\text { oei } \\
\text { nent }\end{array}$ & & \\
\hline$t$ & $\boldsymbol{h}_{\text {top }}$ & $\boldsymbol{h}_{d o m}$ & $\boldsymbol{d}_{d o m}$ & $S \%$ & $N$ & $G$ & $d_{g}$ & $\boldsymbol{h}_{g}$ & $v$ & $N$ & $G$ & $d_{g}$ & $v$ & $N$ & $G$ & $d_{g}$ & $\boldsymbol{h}_{g}$ & $v$ & $I c_{G}$ & $I m_{G}$ & $I c_{v}$ & $I m_{V}$ & $t$ \\
\hline 5 & 0.7 & 0.6 & & & 5000 & & & & 0 & & & & & 5000 & & & & 0 & & & 0.0 & 0.0 & 5 \\
\hline 10 & 1.8 & 1.5 & 2.3 & & 5000 & 0.9 & 1.5 & 1.5 & 1 & & & & & 5000 & 0.9 & 1.5 & 1.5 & 1 & 0.62 & 0.09 & 0.9 & 0.1 & 10 \\
\hline 15 & 3.2 & 2.9 & 5.5 & & 5000 & 5.4 & 3.7 & 2.5 & 10 & & & & & 5000 & 5.4 & 3.7 & 2.5 & 10 & 1.11 & 0.36 & 2.7 & 0.7 & 15 \\
\hline 20 & 4.6 & 4.3 & 8.1 & & 5000 & 11.8 & 5.5 & 3.7 & 29 & & & & & 5000 & 11.8 & 5.5 & 3.7 & 29 & 1.43 & 0.59 & 5.1 & 1.5 & 20 \\
\hline 25 & 6.0 & 5.8 & 10.5 & & 5000 & 19.6 & 7.1 & 4.8 & 61 & & & & & 5000 & 19.6 & 7.1 & 4.8 & 61 & 1.65 & 0.78 & 7.5 & 2.4 & 25 \\
\hline 30 & 7.5 & 7.2 & 13.0 & 22.0 & 5000 & 28.4 & 8.5 & 6.1 & 112 & 752 & 2.5 & 6.5 & 10 & 4248 & 25.9 & 8.8 & 6.1 & 102 & 1.85 & 0.95 & 13.7 & 3.7 & 30 \\
\hline 35 & 8.9 & 8.6 & 16.0 & 22.0 & 4248 & 34.7 & 10.2 & 7.5 & 160 & 1241 & 6.2 & 8.0 & 29 & 3007 & 28.5 & 11.0 & 7.5 & 131 & 1.66 & 1.06 & 11.8 & 4.9 & 35 \\
\hline 40 & 10.3 & 10.0 & 18.7 & 22.7 & 3007 & 36.4 & 12.4 & 8.9 & 190 & 888 & 6.9 & 10.0 & 37 & 2119 & 29.5 & 13.3 & 8.9 & 153 & 1.52 & 1.13 & 12.0 & 5.7 & 40 \\
\hline 45 & 11.6 & 11.3 & 20.9 & 23.5 & 2119 & 36.8 & 14.9 & 10.2 & 213 & 552 & 6.4 & 12.2 & 38 & 1567 & 30.4 & 15.7 & 10.3 & 175 & 1.40 & 1.16 & 12.0 & 6.4 & 45 \\
\hline 50 & 12.8 & 12.5 & 23.6 & 24.2 & 1567 & 37.1 & 17.4 & 11.4 & 235 & 363 & 6.0 & 14.5 & 39 & 1204 & 31.1 & 18.1 & 11.5 & 196 & 1.30 & 1.18 & 11.9 & 7.0 & 50 \\
\hline 55 & 14.0 & 13.7 & 26.2 & 24.9 & 1204 & 37.4 & 19.9 & 12.6 & 255 & 250 & 5.6 & 16.9 & 39 & 953 & 31.8 & 20.6 & 12.7 & 216 & 1.22 & 1.19 & 11.7 & 7.4 & 55 \\
\hline 60 & 15.0 & 14.8 & 28.8 & 25.7 & 953 & 37.7 & 22.4 & 13.7 & 274 & 179 & 5.3 & 19.5 & 40 & 774 & 32.3 & 23.1 & 13.8 & 234 & 1.15 & 1.19 & 11.6 & 7.8 & 60 \\
\hline 65 & 16.1 & 15.8 & 31.3 & 26.4 & 774 & 37.9 & 25.0 & 14.7 & 291 & 132 & 5.1 & 22.1 & 40 & 642 & 32.9 & 25.5 & 14.8 & 252 & 1.09 & 1.18 & 11.3 & 8.1 & 65 \\
\hline 70 & 17.0 & 16.8 & 33.7 & 27.1 & 642 & 38.2 & 27.5 & 15.6 & 308 & 100 & 4.8 & 24.7 & 40 & 542 & 33.3 & 28.0 & 15.7 & 268 & 1.03 & 1.17 & 11.1 & 8.3 & 70 \\
\hline 75 & 17.9 & 17.6 & 36.1 & 27.9 & 542 & 38.4 & 30.0 & 16.4 & 323 & 77 & 4.6 & 27.5 & 40 & 465 & 33.8 & 30.4 & 16.6 & 283 & 0.98 & 1.16 & 10.8 & 8.5 & 75 \\
\hline 80 & 18.7 & 18.5 & 38.4 & 28.6 & 465 & 38.6 & 32.5 & 17.2 & 337 & 61 & 4.4 & 30.2 & 39 & 403 & 34.2 & 32.8 & 17.4 & 297 & 0.94 & 1.15 & 10.6 & 8.6 & 80 \\
\hline 85 & 19.5 & 19.2 & 40.6 & 29.3 & 403 & 38.8 & 35.0 & 17.9 & 350 & 49 & 4.2 & 33.1 & 39 & 354 & 34.6 & 35.2 & 18.1 & 311 & 0.87 & 1.14 & 10.1 & 8.7 & 85 \\
\hline 90 & 20.2 & 19.9 & 42.7 & 30.1 & 354 & 38.7 & 37.3 & 18.6 & 359 & 40 & 4.0 & 35.8 & 38 & 314 & 34.7 & 37.5 & 18.7 & 321 & 0.80 & 1.12 & 9.4 & 8.8 & 90 \\
\hline
\end{tabular}




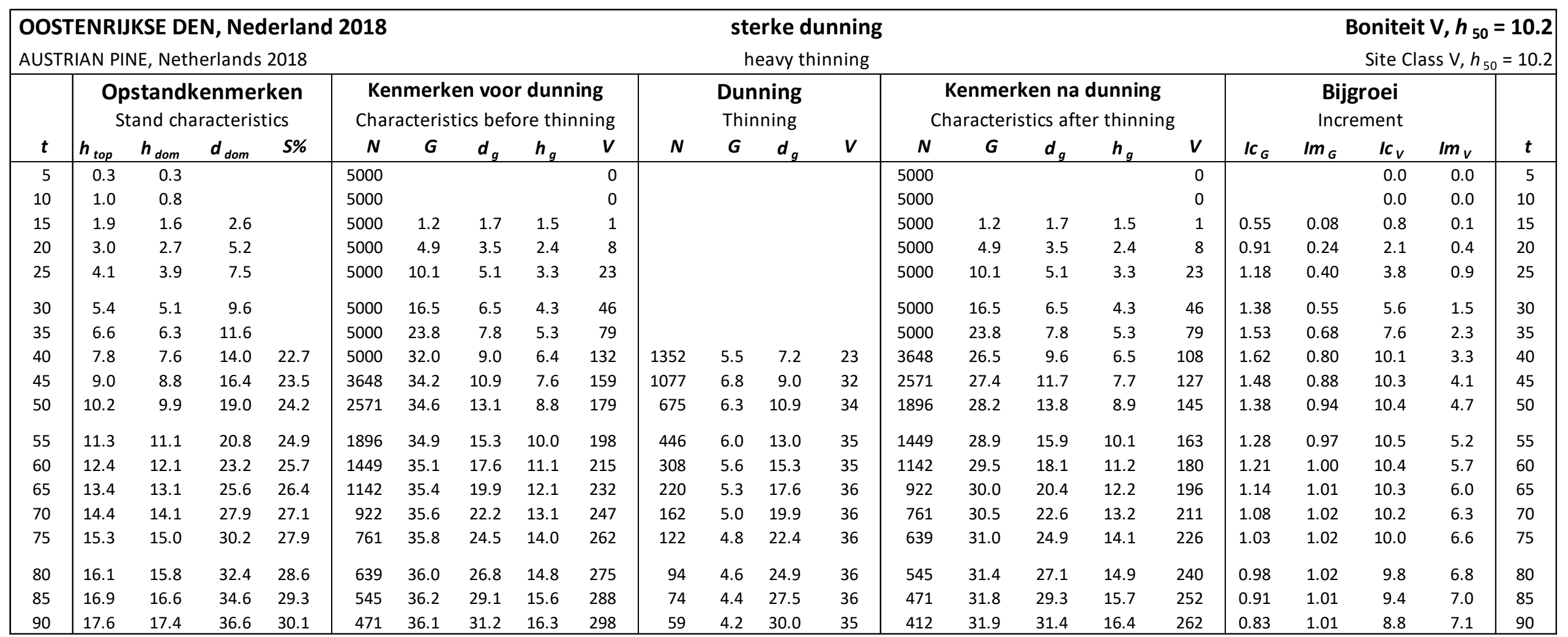




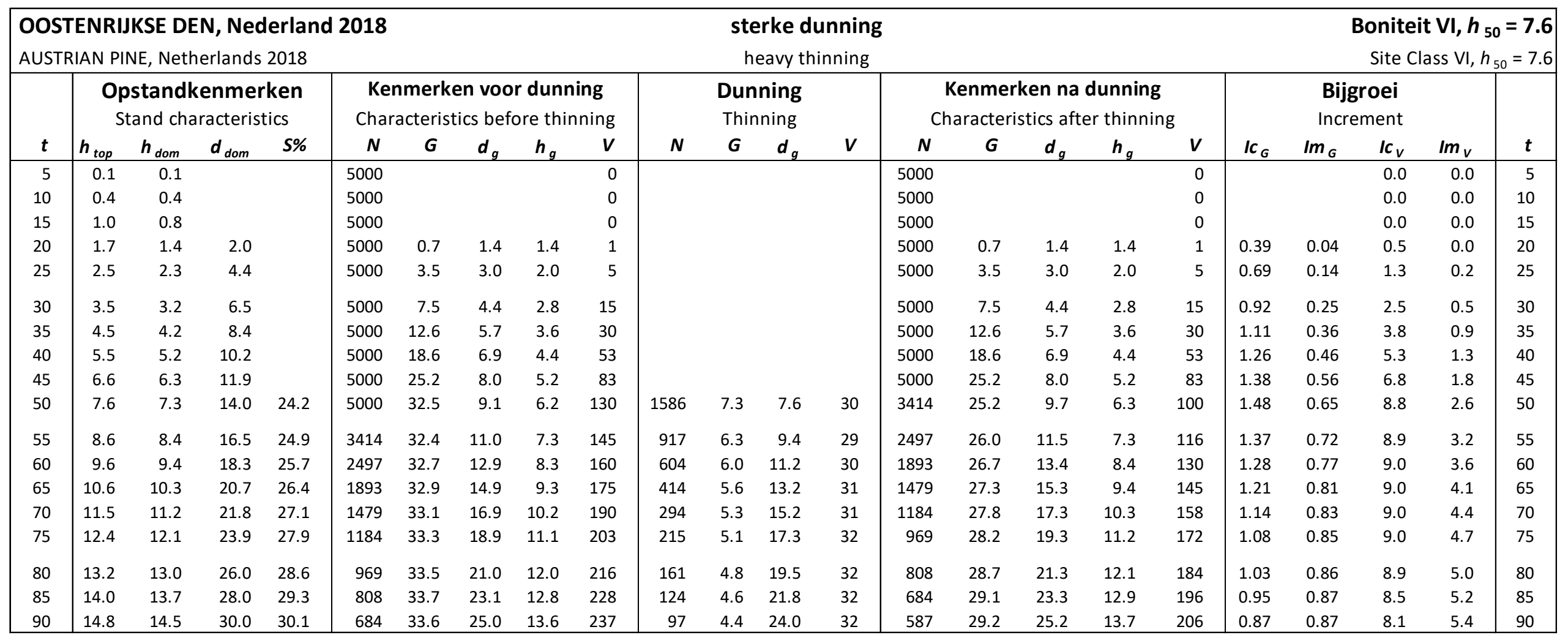




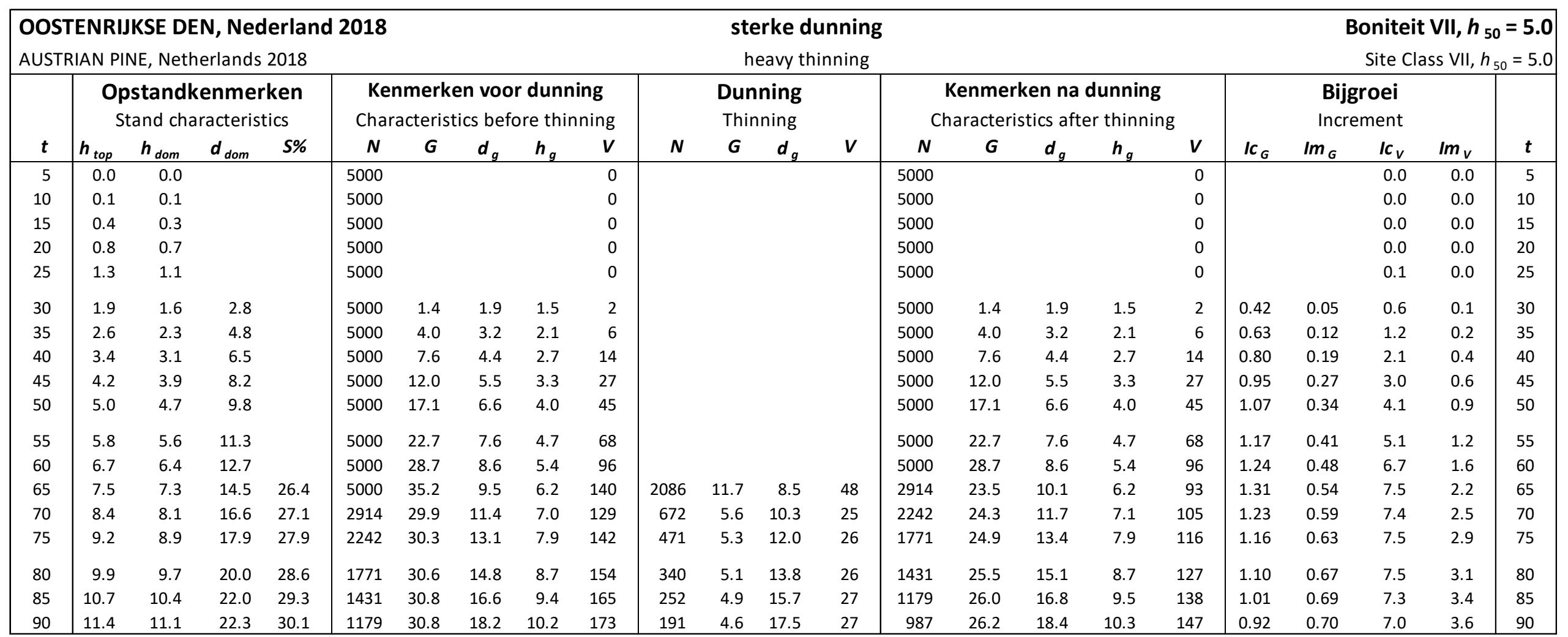




\section{Douglas (Pseudotsuga menziesii)}

Douglas fir

Jansen, J.J., H. Schoonderwoerd, G.M.J. Mohren, E.A.H. Thomassen en J. den Ouden

Bron: Jansen, J.J., H. Schoonderwoerd, G.M.J. Mohren en J. den Ouden, 2016. Groei en productie van douglas in Nederland.

Becking's dunningproeven ontsloten. Wageningen Academic Publishers, $180 \mathrm{blz}$.

Dit rapport is gratis te downloaden op:

https://doi.org/10.3920/978-90-8686-827-8

In het rapport worden twee productieniveaus onderscheiden, een laag niveau tot en met 1980 en een hoog niveau vanaf 1981. In het rapport worden meerdere opbrengsttabellen gepresenteerd, die alle met het gemiddelde productieniveau zijn berekend.

In dit opbrengsttabellenboek is gekozen om alleen tabellen met het hoge productieniveau te presenteren.

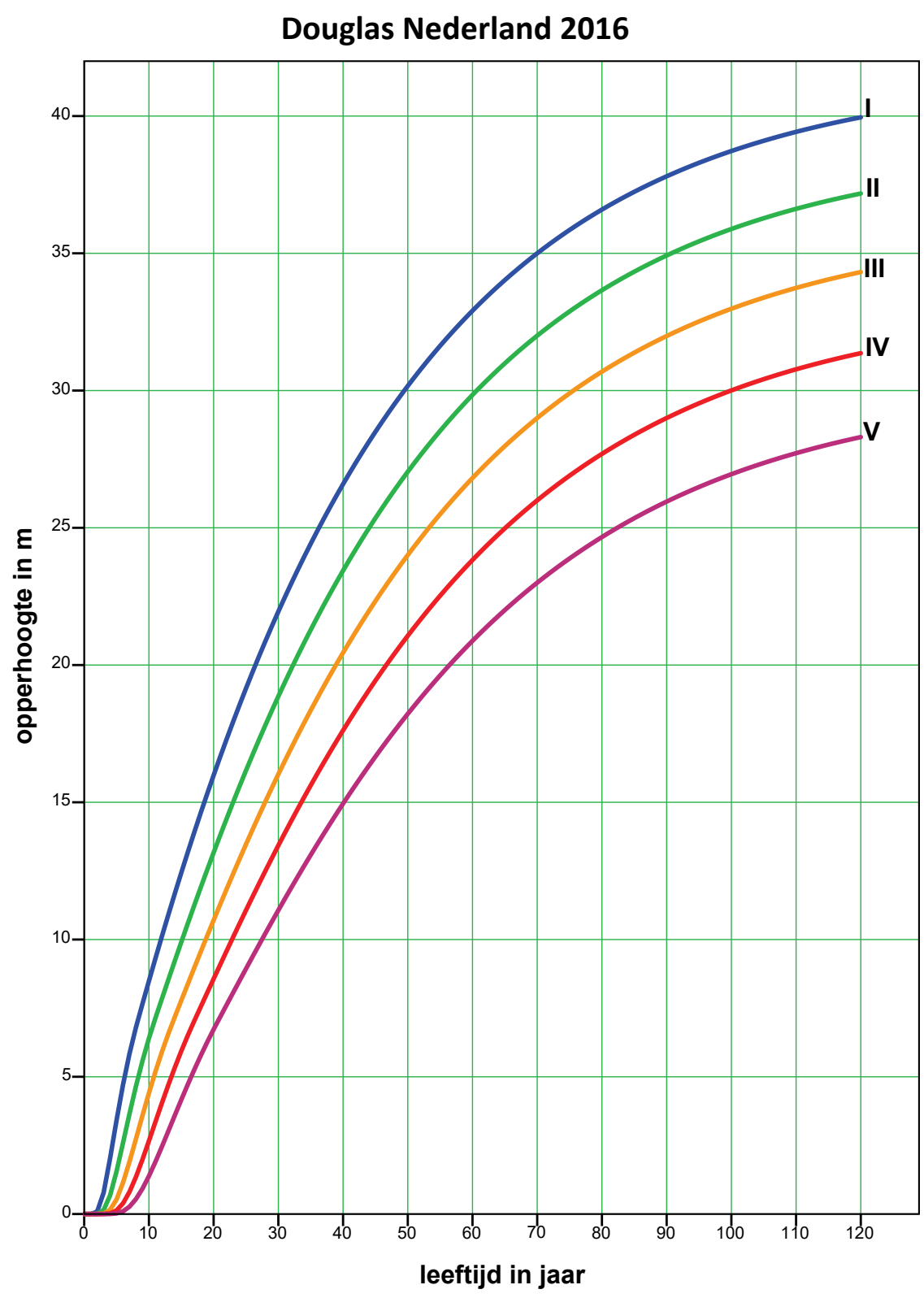




\begin{tabular}{|c|c|c|c|c|c|c|c|c|c|c|c|c|c|c|c|c|c|c|c|c|c|c|c|}
\hline \multicolumn{5}{|c|}{$\begin{array}{l}\text { DOUGLAS, Nederland } 2018 \\
\text { DOUGLAS FIR, Netherlands } 2018\end{array}$} & \multicolumn{15}{|c|}{$\begin{array}{l}\text { matige dunning } \\
\text { moderate thinning }\end{array}$} & \multicolumn{4}{|c|}{$\begin{array}{r}\text { Boniteit I, } \boldsymbol{h}_{70}=\mathbf{3 5 . 0} \\
\text { Site Class I, } h_{70}=35.0\end{array}$} \\
\hline \multirow[b]{2}{*}{$t$} & \multicolumn{4}{|c|}{$\begin{array}{l}\text { Opstandkenmerken } \\
\text { Stand characteristics }\end{array}$} & \multicolumn{5}{|c|}{$\begin{array}{l}\text { Kenmerken voor dunning } \\
\text { Characteristics before thinning }\end{array}$} & \multicolumn{4}{|c|}{$\begin{array}{l}\text { Dunning } \\
\text { Thinning }\end{array}$} & \multicolumn{5}{|c|}{$\begin{array}{c}\text { Kenmerken na dunning } \\
\text { Characteristics after thinning }\end{array}$} & \multicolumn{4}{|c|}{$\begin{array}{l}\text { Bijgroei } \\
\text { Increment }\end{array}$} & \multirow[b]{2}{*}{$t$} \\
\hline & $h_{\text {top }}$ & $\boldsymbol{h}_{\text {dom }}$ & $\boldsymbol{d}_{d o m}$ & S\% & $N$ & $\boldsymbol{G}$ & $d_{g}$ & $h_{g}$ & $v$ & $N$ & $\boldsymbol{G}$ & $d_{g}$ & $v$ & $N$ & $G$ & $d_{g}$ & $h_{g}$ & $v$ & $I c_{G}$ & $I m_{G}$ & $I c_{v}$ & $I m_{V}$ & \\
\hline 5 & 3.4 & 3.4 & 3.9 & & 5000 & 0.7 & 1.3 & 2.7 & 2 & & & & & 5000 & 0.7 & 1.3 & 2.7 & 2 & 1.17 & 0.14 & 3.5 & 0.4 & 5 \\
\hline 10 & 8.5 & 8.4 & 11.8 & 19.0 & 5000 & 19.7 & 7.1 & 6.0 & 99 & 559 & 1.3 & 5.3 & 6 & 4441 & 18.4 & 7.3 & 6.1 & 93 & 5.83 & 1.97 & 35.4 & 9.9 & 10 \\
\hline 15 & 12.4 & 12.3 & 17.9 & 19.0 & 4441 & 38.2 & 10.5 & 10.3 & 247 & 2371 & 12.7 & 8.3 & 82 & 2069 & 25.5 & 12.5 & 10.4 & 165 & 2.91 & 2.63 & 27.0 & 16.9 & 15 \\
\hline 20 & 16.0 & 15.9 & 23.0 & 19.0 & 2069 & 38.8 & 15.4 & 14.0 & 296 & 818 & 9.5 & 12.2 & 73 & 1252 & 29.2 & 17.2 & 14.2 & 223 & 2.43 & 2.64 & 25.7 & 19.2 & 20 \\
\hline 25 & 19.1 & 19.0 & 27.9 & 19.0 & 1252 & 40.4 & 20.3 & 17.3 & 348 & 380 & 7.7 & 16.1 & 66 & 872 & 32.7 & 21.9 & 17.5 & 282 & 2.07 & 2.56 & 24.3 & 20.4 & 25 \\
\hline 30 & 21.9 & 21.8 & 32.5 & 19.0 & 872 & 42.3 & 24.9 & 20.1 & 399 & 208 & 6.5 & 19.9 & 61 & 664 & 35.9 & 26.2 & 20.4 & 338 & 1.79 & 2.45 & 22.8 & 20.9 & 30 \\
\hline 35 & 24.4 & 24.2 & 36.7 & 19.0 & 664 & 44.2 & 29.1 & 22.6 & 448 & 128 & 5.5 & 23.5 & 56 & 536 & 38.7 & 30.3 & 22.9 & 392 & 1.56 & 2.34 & 21.2 & 21.0 & 35 \\
\hline 40 & 26.6 & 26.4 & 40.5 & 19.0 & 536 & 46.0 & 33.0 & 24.8 & 494 & 84 & 4.8 & 26.9 & 51 & 452 & 41.2 & 34.1 & 25.1 & 443 & 1.37 & 2.23 & 19.6 & 21.0 & 40 \\
\hline 45 & 28.5 & 28.2 & 43.9 & 19.0 & 452 & 47.7 & 36.6 & 26.8 & 537 & 58 & 4.2 & 30.1 & 47 & 394 & 43.5 & 37.5 & 27.1 & 490 & 1.21 & 2.12 & 18.1 & 20.7 & 45 \\
\hline 50 & 30.2 & 29.9 & 47.0 & 19.0 & 394 & 49.2 & 39.9 & 28.5 & 577 & 42 & 3.6 & 33.1 & 43 & 351 & 45.6 & 40.6 & 28.8 & 534 & 1.08 & 2.03 & 16.7 & 20.4 & 50 \\
\hline 55 & 31.6 & 31.3 & 49.9 & 19.1 & 351 & 50.7 & 42.8 & 29.9 & 614 & 36 & 3.7 & 36.0 & 44 & 315 & 47.0 & 43.6 & 30.3 & 570 & 0.96 & 1.93 & 15.3 & 20.0 & 55 \\
\hline 60 & 32.9 & 32.6 & 52.5 & 19.3 & 315 & 51.6 & 45.6 & 31.3 & 643 & 28 & 3.3 & 38.7 & 41 & 288 & 48.3 & 46.2 & 31.6 & 602 & 0.87 & 1.85 & 14.1 & 19.5 & 60 \\
\hline 65 & 34.0 & 33.7 & 54.9 & 19.4 & 288 & 52.4 & 48.2 & 32.4 & 669 & 22 & 3.0 & 41.3 & 38 & 265 & 49.4 & 48.7 & 32.8 & 631 & 0.78 & 1.77 & 12.9 & 19.1 & 65 \\
\hline 70 & 35.0 & 34.7 & 57.1 & 19.5 & 265 & 53.2 & 50.5 & 33.4 & 693 & 18 & 2.7 & 43.8 & 35 & 248 & 50.5 & 51.0 & 33.8 & 658 & 0.71 & 1.70 & 11.9 & 18.6 & 70 \\
\hline 75 & 35.9 & 35.5 & 59.1 & 19.6 & 248 & 53.9 & 52.6 & 34.3 & 715 & 15 & 2.5 & 46.1 & 33 & 233 & 51.4 & 53.0 & 34.7 & 683 & 0.65 & 1.63 & 10.9 & 18.1 & 75 \\
\hline 80 & 36.6 & 36.3 & 60.9 & 19.8 & 233 & 54.5 & 54.6 & 35.0 & 735 & 12 & 2.2 & 48.4 & 30 & 221 & 52.3 & 54.9 & 35.4 & 705 & 0.60 & 1.57 & 10.1 & 17.6 & 80 \\
\hline 85 & 37.2 & 37.0 & 62.6 & 19.9 & 221 & 55.1 & 56.4 & 35.7 & 754 & 10 & 2.1 & 50.5 & 28 & 210 & 53.1 & 56.7 & 36.1 & 725 & 0.55 & 1.51 & 9.4 & 17.2 & 85 \\
\hline 90 & 37.8 & 37.6 & 64.1 & 20.0 & 210 & 55.7 & 58.1 & 36.3 & 771 & 9 & 1.9 & 52.5 & 26 & 201 & 53.8 & 58.3 & 36.7 & 744 & 0.51 & 1.45 & 8.7 & 16.7 & 90 \\
\hline 95 & 38.3 & 38.1 & 65.6 & 20.2 & 201 & 56.3 & 59.7 & 36.8 & 786 & 8 & 1.8 & 54.4 & 25 & 194 & 54.5 & 59.8 & 37.2 & 761 & 0.48 & 1.40 & 8.1 & 16.3 & 95 \\
\hline 100 & 38.7 & 38.5 & 66.9 & 20.3 & 194 & 56.8 & 61.1 & 37.2 & 801 & 7 & 1.7 & 56.3 & 23 & 187 & 55.2 & 61.3 & 37.6 & 777 & 0.45 & 1.36 & 7.6 & 15.9 & 100 \\
\hline 105 & 39.1 & 38.9 & 68.2 & 20.4 & 187 & 57.3 & 62.5 & 37.6 & 814 & 6 & 1.6 & 58.1 & 22 & 181 & 55.8 & 62.6 & 38.0 & 792 & 0.42 & 1.31 & 7.2 & 15.5 & 105 \\
\hline 110 & 39.4 & 39.2 & 69.4 & 20.5 & 181 & 57.9 & 63.7 & 37.9 & 827 & 5 & 1.5 & 59.9 & 21 & 176 & 56.4 & 63.9 & 38.4 & 806 & 0.40 & 1.27 & 6.8 & 15.1 & 110 \\
\hline 115 & 39.7 & 39.5 & 70.5 & 20.7 & 176 & 58.3 & 65.0 & 38.2 & 839 & 5 & 1.4 & 61.5 & 20 & 171 & 57.0 & 65.0 & 38.7 & 819 & 0.38 & 1.23 & 6.4 & 14.7 & 115 \\
\hline 120 & 40.0 & 39.8 & 71.5 & 20.8 & 171 & 58.8 & 66.1 & 38.5 & 850 & 4 & 1.3 & 63.2 & 19 & 167 & 57.5 & 66.2 & 38.9 & 831 & 0.36 & 1.20 & 6.1 & 14.4 & 120 \\
\hline
\end{tabular}




\begin{tabular}{|c|c|c|c|c|c|c|c|c|c|c|c|c|c|c|c|c|c|c|c|c|c|c|c|}
\hline \multicolumn{5}{|c|}{$\begin{array}{l}\text { DOUGLAS, Nederland } 2018 \\
\text { DOUGLAS FIR, Netherlands } 2018\end{array}$} & \multicolumn{15}{|c|}{ matige dunning } & \multicolumn{4}{|c|}{$\begin{array}{r}\text { Boniteit II, } \boldsymbol{h}_{70}=\mathbf{3 2 . 0} \\
\text { Site Class II, } h_{70}=32.0\end{array}$} \\
\hline \multirow[b]{2}{*}{$t$} & \multicolumn{4}{|c|}{$\begin{array}{l}\text { Opstandkenmerken } \\
\text { Stand characteristics }\end{array}$} & \multicolumn{5}{|c|}{$\begin{array}{l}\text { Kenmerken voor dunning } \\
\text { Characteristics before thinning }\end{array}$} & \multicolumn{4}{|c|}{$\begin{array}{l}\text { Dunning } \\
\text { Thinning }\end{array}$} & \multicolumn{5}{|c|}{$\begin{array}{c}\text { Kenmerken na dunning } \\
\text { Characteristics after thinning }\end{array}$} & \multicolumn{4}{|c|}{$\begin{array}{l}\text { Bijgroei } \\
\text { Increment }\end{array}$} & \multirow[b]{2}{*}{$t$} \\
\hline & $h_{\text {top }}$ & $\boldsymbol{h}_{\text {dom }}$ & $\boldsymbol{d}_{\text {dom }}$ & S\% & $N$ & $\boldsymbol{G}$ & $d_{g}$ & $\boldsymbol{h}_{g}$ & $v$ & $N$ & $\boldsymbol{G}$ & $d_{g}$ & $v$ & $N$ & $\boldsymbol{G}$ & $d_{g}$ & $\boldsymbol{h}_{g}$ & $v$ & $\mid c_{G}$ & $I m_{G}$ & $I c_{V}$ & $I m_{V}$ & \\
\hline 5 & 1.6 & 1.5 & 1.8 & & 5000 & 0.0 & 0.3 & 1.2 & 0 & & & & & 5000 & 0.0 & 0.3 & 1.2 & 0 & 0.11 & 0.01 & 0.2 & 0.0 & 5 \\
\hline 10 & 6.4 & 6.4 & 8.0 & & 5000 & 6.5 & 4.1 & 5.1 & 27 & & & & & 5000 & 6.5 & 4.1 & 5.1 & 27 & 2.51 & 0.65 & 13.6 & 2.7 & 10 \\
\hline 15 & 9.9 & 9.8 & 14.7 & 19.0 & 5000 & 28.7 & 8.5 & 7.6 & 159 & 1740 & 5.8 & 6.5 & 32 & 3260 & 22.9 & 9.5 & 7.7 & 127 & 3.73 & 1.91 & 26.9 & 10.6 & 15 \\
\hline 20 & 13.2 & 13.1 & 19.5 & 19.0 & 3260 & 36.7 & 12.0 & 11.1 & 244 & 1415 & 9.6 & 9.3 & 64 & 1845 & 27.1 & 13.7 & 11.2 & 181 & 2.43 & 2.12 & 22.8 & 13.8 & 20 \\
\hline 25 & $\mid 16.2$ & 16.0 & 24.1 & 19.0 & 1845 & 38.4 & 16.3 & 14.2 & 293 & 620 & 7.8 & 12.7 & 59 & 1225 & 30.6 & 17.8 & 14.3 & 233 & 2.09 & 2.15 & 22.1 & 15.5 & 25 \\
\hline 30 & 18.9 & 18.7 & 28.4 & 19.0 & 1225 & 40.4 & 20.5 & 17.0 & 341 & 326 & 6.6 & 16.0 & 56 & 899 & 33.8 & 21.9 & 17.2 & 285 & 1.82 & 2.12 & 21.1 & 16.5 & 30 \\
\hline 35 & 21.3 & 21.1 & 32.4 & 19.0 & 899 & 42.3 & 24.5 & 19.5 & 388 & 193 & 5.7 & 19.3 & 52 & 706 & 36.7 & 25.7 & 19.7 & 336 & 1.60 & 2.06 & 19.9 & 17.1 & 35 \\
\hline 40 & 23.4 & 23.2 & 36.1 & 19.0 & 706 & 44.2 & 28.2 & 21.7 & 432 & 124 & 4.9 & 22.5 & 48 & 582 & 39.2 & 29.3 & 21.9 & 384 & 1.41 & 1.99 & 18.6 & 17.4 & 40 \\
\hline 45 & 25.4 & 25.1 & 39.4 & 19.0 & 582 & 45.9 & 31.7 & 23.6 & 473 & 85 & 4.3 & 25.5 & 45 & 498 & 41.6 & 32.6 & 23.9 & 429 & 1.26 & 1.92 & 17.3 & 17.4 & 45 \\
\hline 50 & 27.0 & 26.8 & 42.5 & 19.0 & 498 & 47.5 & 34.9 & 25.3 & 512 & 60 & 3.8 & 28.4 & 41 & 437 & 43.7 & 35.7 & 25.6 & 471 & 1.12 & 1.84 & 16.1 & 17.4 & 50 \\
\hline 55 & 28.5 & 28.3 & 45.4 & 19.1 & 437 & 49.0 & 37.8 & 26.8 & 548 & 50 & 3.8 & 31.1 & 42 & 388 & 45.2 & 38.5 & 27.1 & 506 & 1.01 & 1.77 & 14.8 & 17.2 & 55 \\
\hline 60 & 29.8 & 29.6 & 48.0 & 19.3 & 388 & 50.0 & 40.5 & 28.2 & 577 & 38 & 3.4 & 33.8 & 39 & 350 & 46.6 & 41.2 & 28.5 & 538 & 0.90 & 1.70 & 13.7 & 16.9 & 60 \\
\hline 65 & 31.0 & 30.7 & 50.3 & 19.4 & 350 & 50.9 & 43.0 & 29.3 & 603 & 30 & 3.1 & 36.3 & 36 & 320 & 47.8 & 43.6 & 29.7 & 567 & 0.82 & 1.64 & 12.6 & 16.6 & 65 \\
\hline 70 & 32.0 & 31.7 & 52.5 & 19.5 & 320 & 51.7 & 45.4 & 30.4 & 627 & 24 & 2.8 & 38.6 & 34 & 296 & 48.9 & 45.9 & 30.7 & 593 & 0.74 & 1.58 & 11.6 & 16.3 & 70 \\
\hline 75 & 32.9 & 32.6 & 54.5 & 19.6 & 296 & 52.5 & 47.5 & 31.3 & 649 & 19 & 2.5 & 40.9 & 31 & 277 & 49.9 & 47.9 & 31.6 & 618 & 0.68 & 1.52 & 10.7 & 16.0 & 75 \\
\hline 80 & 33.7 & 33.4 & 56.3 & 19.8 & 277 & 53.2 & 49.4 & 32.1 & 669 & 16 & 2.3 & 43.0 & 29 & 261 & 50.8 & 49.8 & 32.4 & 639 & 0.62 & 1.47 & 9.8 & 15.6 & 80 \\
\hline 85 & \begin{tabular}{|l}
34.3 \\
\end{tabular} & 34.0 & 58.0 & 19.9 & 261 & 53.8 & 51.3 & 32.8 & 687 & 13 & 2.1 & 45.1 & 27 & 247 & 51.7 & 51.6 & 33.1 & 660 & 0.57 & 1.41 & 9.1 & 15.2 & 85 \\
\hline 90 & 34.9 & 34.6 & 59.5 & 20.0 & 247 & 54.4 & 52.9 & 33.4 & 703 & 11 & 2.0 & 47.0 & 25 & 236 & 52.4 & 53.2 & 33.8 & 678 & 0.53 & 1.37 & 8.5 & 14.9 & 90 \\
\hline 95 & 35.4 & 35.1 & 61.0 & 20.2 & 236 & 55.0 & 54.5 & 33.9 & 719 & 10 & 1.8 & 48.9 & 24 & 226 & 53.2 & 54.7 & 34.3 & 695 & 0.49 & 1.32 & 7.9 & 14.5 & 95 \\
\hline 100 & 35.9 & 35.6 & 62.3 & 20.3 & 226 & 55.6 & 55.9 & 34.4 & 733 & 8 & 1.7 & 50.7 & 22 & 218 & 53.9 & 56.1 & 34.8 & 711 & 0.46 & 1.28 & 7.4 & 14.2 & 100 \\
\hline 105 & 36.3 & 36.0 & 63.5 & 20.4 & 218 & 56.1 & 57.3 & 34.8 & 746 & 7 & 1.6 & 52.4 & 21 & 211 & 54.5 & 57.4 & 35.2 & 725 & 0.44 & 1.24 & 6.9 & 13.8 & 105 \\
\hline 110 & 36.6 & 36.4 & 64.7 & 20.5 & 211 & 56.6 & 58.5 & 35.1 & 758 & 6 & 1.5 & 54.1 & 20 & 204 & 55.1 & 58.7 & 35.5 & 738 & 0.41 & 1.20 & 6.5 & 13.5 & 110 \\
\hline 115 & 36.9 & 36.7 & 65.8 & 20.7 & 204 & 57.2 & 59.7 & 35.4 & 770 & 6 & 1.4 & 55.7 & 19 & 198 & 55.7 & 59.8 & 35.9 & 751 & 0.39 & 1.17 & 6.1 & 13.2 & 115 \\
\hline 120 & 37.2 & 37.0 & 66.8 & 20.8 & 198 & 57.7 & 60.8 & 35.7 & 781 & 5 & 1.3 & 57.3 & 18 & 193 & 56.3 & 60.9 & 36.1 & 763 & 0.37 & 1.13 & 5.8 & 12.9 & 120 \\
\hline
\end{tabular}




\begin{tabular}{|c|c|c|c|c|c|c|c|c|c|c|c|c|c|c|c|c|c|c|c|c|c|c|c|}
\hline \multicolumn{5}{|c|}{$\begin{array}{l}\text { DOUGLAS, Nederland } 2018 \\
\text { DOUGLAS FIR, Netherlands } 2018\end{array}$} & \multicolumn{15}{|c|}{$\begin{array}{l}\text { matige dunning } \\
\text { moderate thinning }\end{array}$} & \multicolumn{4}{|c|}{$\begin{array}{r}\text { Boniteit III, } \boldsymbol{h}_{70}=\mathbf{2 9 . 0} \\
\text { Site Class III, } h_{70}=29.0\end{array}$} \\
\hline \multirow[b]{2}{*}{$t$} & \multicolumn{4}{|c|}{$\begin{array}{l}\text { Opstandkenmerken } \\
\text { Stand characteristics }\end{array}$} & \multicolumn{5}{|c|}{$\begin{array}{l}\text { Kenmerken voor dunning } \\
\text { Characteristics before thinning }\end{array}$} & \multicolumn{4}{|c|}{$\begin{array}{l}\text { Dunning } \\
\text { Thinning }\end{array}$} & \multicolumn{5}{|c|}{$\begin{array}{l}\text { Kenmerken na dunning } \\
\text { Characteristics after thinning }\end{array}$} & \multicolumn{4}{|c|}{$\begin{array}{l}\text { Bijgroei } \\
\text { Increment }\end{array}$} & \multirow[b]{2}{*}{$t$} \\
\hline & $h_{\text {top }}$ & $\boldsymbol{h}_{\text {dom }}$ & $\boldsymbol{d}_{d o m}$ & S\% & $N$ & $\boldsymbol{G}$ & $d_{g}$ & $h_{g}$ & $v$ & $N$ & $\boldsymbol{G}$ & $d_{g}$ & $v$ & $N$ & $\boldsymbol{G}$ & $d_{g}$ & $h_{g}$ & $v$ & $I c_{G}$ & $I m_{G}$ & $I c_{v}$ & $I m_{V}$ & \\
\hline 5 & & & & & 5000 & & & & 0 & & & & & 5000 & & & & 0 & & & 0.0 & 0.0 & 5 \\
\hline 10 & 4.4 & 4.4 & 5.6 & & 5000 & 1.9 & 2.2 & 3.5 & 6 & & & & & 5000 & 1.9 & 2.2 & 3.5 & 6 & 1.26 & 0.19 & 4.3 & 0.6 & 10 \\
\hline 15 & 7.8 & 7.7 & 11.0 & 19.5 & 5000 & 14.5 & 6.1 & 5.2 & 68 & & & & & 5000 & 14.5 & 6.1 & 5.3 & 68 & 4.62 & 0.96 & 24.9 & 4.5 & 15 \\
\hline 20 & 10.7 & 10.6 & 16.4 & 19.0 & 5000 & 32.7 & 9.1 & 8.4 & 188 & 2209 & 8.3 & 6.9 & 48 & 2791 & 24.4 & 10.5 & 8.5 & 140 & 2.51 & 1.63 & 20.2 & 9.4 & 20 \\
\hline 25 & 13.5 & 13.4 & 20.5 & 19.0 & 2791 & 35.6 & 12.7 & 11.4 & 238 & 1028 & 7.6 & 9.7 & 51 & 1763 & 28.0 & 14.2 & 11.5 & 187 & 2.09 & 1.75 & 19.5 & 11.4 & 25 \\
\hline 30 & 16.0 & 15.9 & 24.5 & 19.0 & 1763 & 37.8 & 16.5 & 14.1 & 283 & 517 & 6.5 & 12.7 & 49 & 1246 & 31.2 & 17.9 & 14.2 & 234 & 1.84 & 1.79 & 19.0 & 12.7 & 30 \\
\hline 35 & 18.3 & 18.2 & 28.3 & 19.0 & 1246 & 39.9 & 20.2 & 16.5 & 327 & 296 & 5.7 & 15.6 & 47 & 950 & 34.2 & 21.4 & 16.6 & 281 & 1.63 & 1.78 & 18.2 & 13.6 & 35 \\
\hline 40 & 20.4 & 20.3 & 31.9 & 19.0 & 950 & 41.9 & 23.7 & 18.6 & 369 & 185 & 5.0 & 18.5 & 44 & 765 & 36.9 & 24.8 & 18.8 & 325 & 1.44 & 1.75 & 17.3 & 14.1 & 40 \\
\hline 45 & 22.3 & 22.1 & 35.1 & 19.0 & 765 & 43.7 & 27.0 & 20.5 & 409 & 123 & 4.4 & 21.3 & 41 & 641 & 39.3 & 27.9 & 20.8 & 368 & 1.29 & 1.71 & 16.3 & 14.4 & 45 \\
\hline 50 & 24.0 & 23.8 & 38.1 & 19.0 & 641 & 45.4 & 30.0 & 22.2 & 447 & 86 & 3.9 & 24.0 & 38 & 555 & 41.5 & 30.9 & 22.5 & 408 & 1.15 & 1.66 & 15.2 & 14.5 & 50 \\
\hline 55 & 25.5 & 25.3 & 40.9 & 19.1 & 555 & 47.0 & 32.8 & 23.8 & 481 & 70 & 3.8 & 26.5 & 39 & 485 & 43.1 & 33.6 & 24.0 & 442 & 1.04 & 1.61 & 14.1 & 14.5 & 55 \\
\hline 60 & 26.8 & 26.6 & 43.5 & 19.3 & 485 & 48.0 & 35.5 & 25.1 & 510 & 52 & 3.5 & 29.0 & 37 & 433 & 44.6 & 36.2 & 25.4 & 473 & 0.93 & 1.55 & 13.0 & 14.4 & 60 \\
\hline 65 & 28.0 & 27.7 & 45.8 & 19.4 & 433 & 49.0 & 38.0 & 26.3 & 536 & 40 & 3.1 & 31.4 & 34 & 393 & 45.9 & 38.6 & 26.6 & 501 & 0.84 & 1.50 & 12.0 & 14.3 & 65 \\
\hline 70 & 29.0 & 28.7 & 47.9 & 19.5 & 393 & 49.9 & 40.2 & 27.4 & 559 & 32 & 2.8 & 33.6 & 32 & 361 & 47.0 & 40.8 & 27.7 & 527 & 0.77 & 1.45 & 11.1 & 14.1 & 70 \\
\hline 75 & 29.9 & 29.6 & 49.9 & 19.6 & 361 & 50.7 & 42.3 & 28.3 & 580 & 26 & 2.6 & 35.8 & 30 & 335 & 48.1 & 42.8 & 28.6 & 551 & 0.70 & 1.40 & 10.2 & 13.9 & 75 \\
\hline 80 & 30.7 & 30.4 & 51.7 & 19.8 & 335 & 51.4 & 44.2 & 29.1 & 600 & 21 & 2.4 & 37.8 & 28 & 314 & 49.1 & 44.6 & 29.4 & 572 & 0.64 & 1.36 & 9.4 & 13.6 & 80 \\
\hline 85 & 31.4 & 31.1 & 53.3 & 19.9 & 314 & 52.1 & 46.0 & 29.8 & 617 & 18 & 2.2 & 39.8 & 26 & 296 & 50.0 & 46.4 & 30.2 & 592 & 0.59 & 1.32 & 8.7 & 13.4 & 85 \\
\hline 90 & 32.0 & 31.7 & 54.9 & 20.0 & 296 & 52.8 & 47.6 & 30.4 & 634 & 15 & 2.0 & 41.6 & 24 & 281 & 50.8 & 47.9 & 30.8 & 610 & 0.55 & 1.27 & 8.1 & 13.1 & 90 \\
\hline 95 & 32.5 & 32.2 & 56.3 & 20.2 & 281 & 53.4 & 49.2 & 31.0 & 649 & 13 & 1.9 & 43.4 & 23 & 269 & 51.6 & 49.4 & 31.3 & 626 & 0.51 & 1.23 & 7.5 & 12.8 & 95 \\
\hline 100 & 33.0 & 32.7 & 57.6 & 20.3 & 269 & 54.0 & 50.6 & 31.5 & 662 & 11 & 1.7 & 45.1 & 21 & 258 & 52.3 & 50.8 & 31.8 & 641 & 0.47 & 1.20 & 7.0 & 12.5 & 100 \\
\hline 105 & 33.4 & 33.1 & 58.8 & 20.4 & 258 & 54.6 & 51.9 & 31.9 & 675 & 9 & 1.6 & 46.8 & 20 & 249 & 53.0 & 52.1 & 32.3 & 655 & 0.45 & 1.16 & 6.5 & 12.2 & 105 \\
\hline 110 & 33.7 & 33.4 & 59.9 & 20.5 & 249 & 55.1 & 53.1 & 32.3 & 687 & 8 & 1.5 & 48.4 & 19 & 240 & 53.6 & 53.3 & 32.6 & 668 & 0.42 & 1.13 & 6.1 & 12.0 & 110 \\
\hline 115 & 34.0 & 33.8 & 61.0 & 20.7 & 240 & 55.7 & 54.3 & 32.6 & 698 & 7 & 1.4 & 49.9 & 18 & 233 & 54.3 & 54.4 & 33.0 & 680 & 0.40 & 1.10 & 5.8 & 11.7 & 115 \\
\hline 120 & 34.3 & 34.0 & 62.0 & 20.8 & 233 & 56.2 & 55.4 & 32.9 & 708 & 6 & 1.3 & 51.4 & 17 & 227 & 54.9 & 55.5 & 33.2 & 691 & 0.38 & 1.07 & 5.5 & 11.5 & 120 \\
\hline
\end{tabular}




\begin{tabular}{|c|c|c|c|c|c|c|c|c|c|c|c|c|c|c|c|c|c|c|c|c|c|c|c|}
\hline \multicolumn{5}{|c|}{$\begin{array}{l}\text { DOUGLAS, Nederland } 2018 \\
\text { DOUGLAS FIR, Netherlands } 2018\end{array}$} & \multicolumn{15}{|c|}{ matige dunning } & \multicolumn{4}{|c|}{$\begin{array}{r}\text { Boniteit IV, } \boldsymbol{h}_{\mathbf{7 0}}=\mathbf{2 6 . 0} \\
\text { Site Class IV, } h_{70}=26.0\end{array}$} \\
\hline \multirow[b]{2}{*}{$t$} & \multicolumn{4}{|c|}{$\begin{array}{l}\text { Opstandkenmerken } \\
\text { Stand characteristics }\end{array}$} & \multicolumn{5}{|c|}{$\begin{array}{l}\text { Kenmerken voor dunning } \\
\text { Characteristics before thinning }\end{array}$} & \multicolumn{4}{|c|}{$\begin{array}{l}\text { Dunning } \\
\text { Thinning }\end{array}$} & \multicolumn{5}{|c|}{$\begin{array}{c}\text { Kenmerken na dunning } \\
\text { Characteristics after thinning }\end{array}$} & \multicolumn{4}{|c|}{$\begin{array}{l}\text { Bijgroei } \\
\text { Increment }\end{array}$} & \multirow[b]{2}{*}{$t$} \\
\hline & $\boldsymbol{h}_{\text {top }}$ & $\boldsymbol{h}_{\text {dom }}$ & $\boldsymbol{d}_{d o m}$ & S\% & $N$ & $\boldsymbol{G}$ & $d_{g}$ & $\boldsymbol{h}_{g}$ & $v$ & $N$ & $\boldsymbol{G}$ & $d_{g}$ & $v$ & $N$ & $\boldsymbol{G}$ & $d_{g}$ & $h_{g}$ & $v$ & $I c_{G}$ & $I m_{G}$ & $I c_{V}$ & $I m_{V}$ & \\
\hline 5 & & & & & 5000 & & & & 0 & & & & & 5000 & & & & 0 & & & 0.0 & 0.0 & 5 \\
\hline 10 & 2.7 & 2.6 & 3.4 & & 5000 & 0.3 & 0.8 & 2.1 & 1 & & & & & 5000 & 0.3 & 0.8 & 2.1 & 1 & 0.28 & 0.03 & 0.7 & 0.1 & 10 \\
\hline 15 & 5.9 & 5.9 & 8.1 & & 5000 & 5.2 & 3.6 & 4.7 & 20 & & & & & 5000 & 5.2 & 3.6 & 4.7 & 20 & 1.51 & 0.35 & 6.4 & 1.3 & 15 \\
\hline 20 & 8.6 & 8.5 & 13.1 & 19.0 & 5000 & 20.4 & 7.2 & 6.1 & 100 & 644 & 1.4 & 5.2 & 7 & 4356 & 19.0 & 7.5 & 6.2 & 93 & 3.60 & 1.02 & 21.4 & 5.0 & 20 \\
\hline 25 & 11.1 & 11.0 & 17.6 & 19.0 & 4356 & 33.0 & 9.8 & 8.8 & 192 & 1744 & 7.4 & 7.3 & 43 & 2612 & 25.6 & 11.2 & 9.0 & 149 & 2.06 & 1.37 & 16.9 & 7.9 & 25 \\
\hline 30 & 13.4 & 13.3 & 21.2 & 19.0 & 2612 & 35.3 & 13.1 & 11.4 & 233 & 838 & 6.4 & 9.9 & 42 & 1774 & 28.9 & 14.4 & 11.5 & 191 & 1.83 & 1.47 & 16.8 & 9.4 & 30 \\
\hline 35 & 15.6 & 15.5 & 24.8 & 19.0 & 1774 & 37.5 & 16.4 & 13.6 & 274 & 463 & 5.6 & 12.4 & 41 & 1312 & 31.9 & 17.6 & 13.8 & 233 & 1.63 & 1.50 & 16.4 & 10.4 & 35 \\
\hline 40 & 17.6 & 17.5 & 28.1 & 19.0 & 1312 & 39.6 & 19.6 & 15.7 & 313 & 281 & 5.0 & 15.0 & 39 & 1030 & 34.7 & 20.7 & 15.9 & 274 & 1.46 & 1.51 & 15.7 & 11.1 & 40 \\
\hline 45 & 19.4 & 19.3 & 31.2 & 19.0 & 1030 & 41.6 & 22.7 & 17.6 & 350 & 183 & 4.4 & 17.5 & 37 & 847 & 37.1 & 23.6 & 17.8 & 313 & 1.31 & 1.50 & 14.9 & 11.6 & 45 \\
\hline 50 & 21.1 & 20.9 & 34.1 & 19.0 & 847 & 43.3 & 25.5 & 19.3 & 386 & 126 & 3.9 & 19.9 & 35 & 721 & 39.4 & 26.4 & 19.5 & 350 & 1.17 & 1.47 & 14.1 & 11.9 & 50 \\
\hline 55 & 22.5 & 22.3 & 36.8 & 19.1 & 721 & 44.9 & 28.2 & 20.8 & 418 & 99 & 3.9 & 22.3 & 36 & 622 & 41.1 & 29.0 & 21.0 & 383 & 1.06 & 1.44 & 13.1 & 12.1 & 55 \\
\hline 60 & \begin{tabular}{|l|}
23.8 \\
\end{tabular} & 23.6 & 39.3 & 19.3 & 622 & 46.1 & 30.7 & 22.1 & 446 & 73 & 3.5 & 24.6 & 34 & 548 & 42.6 & 31.4 & 22.4 & 412 & 0.95 & 1.40 & 12.2 & 12.1 & 60 \\
\hline 65 & 25.0 & 24.8 & 41.5 & 19.4 & 548 & 47.1 & 33.1 & 23.3 & 471 & 56 & 3.2 & 26.8 & 32 & 492 & 44.0 & 33.7 & 23.6 & 439 & 0.86 & 1.36 & 11.3 & 12.1 & 65 \\
\hline 70 & 26.0 & 25.8 & 43.6 & 19.5 & 492 & 48.1 & 35.3 & 24.3 & 493 & 44 & 2.9 & 28.9 & 30 & 449 & 45.2 & 35.8 & 24.6 & 464 & 0.78 & 1.32 & 10.4 & 12.0 & 70 \\
\hline 75 & 26.9 & 26.7 & 45.5 & 19.6 & 449 & 48.9 & 37.3 & 25.3 & 514 & 35 & 2.6 & 30.9 & 28 & 414 & 46.3 & 37.8 & 25.6 & 486 & 0.71 & 1.29 & 9.6 & 11.9 & 75 \\
\hline 80 & \begin{tabular}{|l|l}
27.7 \\
\end{tabular} & 27.5 & 47.2 & 19.8 & 414 & 49.7 & 39.1 & 26.1 & 532 & 28 & 2.4 & 32.8 & 26 & 385 & 47.3 & 39.5 & 26.4 & 507 & 0.66 & 1.25 & 8.9 & 11.7 & 80 \\
\hline 85 & 28.4 & 28.1 & 48.8 & 19.9 & 385 & 50.5 & 40.8 & 26.8 & 549 & 23 & 2.2 & 34.7 & 24 & 362 & 48.3 & 41.2 & 27.1 & 525 & 0.60 & 1.21 & 8.2 & 11.5 & 85 \\
\hline 90 & 29.0 & 28.7 & 50.3 & 20.0 & 362 & 51.2 & 42.4 & 27.4 & 565 & 20 & 2.0 & 36.4 & 22 & 342 & 49.1 & 42.7 & 27.8 & 542 & 0.56 & 1.18 & 7.6 & 11.3 & 90 \\
\hline 95 & 29.5 & 29.3 & 51.7 & 20.2 & 342 & 51.8 & 43.9 & 28.0 & 579 & 17 & 1.9 & 38.1 & 21 & 326 & 49.9 & 44.2 & 28.3 & 558 & 0.52 & 1.14 & 7.1 & 11.1 & 95 \\
\hline 100 & 30.0 & 29.7 & 53.0 & 20.3 & 326 & 52.4 & 45.3 & 28.5 & 592 & 14 & 1.7 & 39.7 & 20 & 312 & 50.7 & 45.5 & 28.8 & 572 & 0.48 & 1.11 & 6.6 & 10.9 & 100 \\
\hline 105 & 30.4 & 30.1 & 54.1 & 20.4 & 312 & 53.0 & 46.5 & 28.9 & 604 & 12 & 1.6 & 41.2 & 19 & 300 & 51.4 & 46.7 & 29.2 & 586 & 0.45 & 1.08 & 6.1 & 10.7 & 105 \\
\hline 110 & 30.8 & 30.5 & 55.2 & 20.5 & 300 & 53.6 & 47.7 & 29.3 & 615 & 11 & 1.5 & 42.7 & 17 & 289 & 52.1 & 47.9 & 29.6 & 598 & 0.43 & 1.05 & 5.7 & 10.5 & 110 \\
\hline 115 & \begin{tabular}{|l|l}
31.1 \\
\end{tabular} & 30.8 & 56.3 & 20.7 & 289 & 54.2 & 48.9 & 29.6 & 626 & 9 & 1.4 & 44.2 & 16 & 280 & 52.8 & 49.0 & 30.0 & 609 & 0.41 & 1.02 & 5.4 & 10.2 & 115 \\
\hline 120 & \begin{tabular}{|l|l}
31.4 \\
\end{tabular} & 31.1 & 57.2 & 20.8 & 280 & 54.7 & 49.9 & 29.9 & 635 & 8 & 1.3 & 45.6 & 16 & 271 & 53.4 & 50.0 & 30.3 & 619 & 0.39 & 1.00 & 5.1 & 10.0 & 120 \\
\hline
\end{tabular}




\begin{tabular}{|c|c|c|c|c|c|c|c|c|c|c|c|c|c|c|c|c|c|c|c|c|c|c|c|}
\hline \multicolumn{5}{|c|}{$\begin{array}{l}\text { DOUGLAS, Nederland } 2018 \\
\text { DOUGLAS FIR, Netherlands } 2018\end{array}$} & \multicolumn{15}{|c|}{ matige dunning } & \multicolumn{4}{|c|}{$\begin{array}{r}\text { Boniteit V, } \boldsymbol{h}_{70}=\mathbf{2 3 . 0} \\
\text { Site Class V, } h_{70}=23.0\end{array}$} \\
\hline \multirow[b]{2}{*}{$t$} & \multicolumn{4}{|c|}{$\begin{array}{l}\text { Opstandkenmerken } \\
\text { Stand characteristics }\end{array}$} & \multicolumn{5}{|c|}{$\begin{array}{l}\text { Kenmerken voor dunning } \\
\text { Characteristics before thinning }\end{array}$} & \multicolumn{4}{|c|}{$\begin{array}{l}\text { Dunning } \\
\text { Thinning }\end{array}$} & \multicolumn{5}{|c|}{$\begin{array}{c}\text { Kenmerken na dunning } \\
\text { Characteristics after thinning }\end{array}$} & \multicolumn{4}{|c|}{$\begin{array}{l}\text { Bijgroei } \\
\text { Increment }\end{array}$} & \multirow[b]{2}{*}{$t$} \\
\hline & $h_{\text {top }}$ & $\boldsymbol{h}_{\text {dom }}$ & $\boldsymbol{d}_{d o m}$ & S\% & $N$ & $\boldsymbol{G}$ & $d_{g}$ & $\boldsymbol{h}_{g}$ & $v$ & $N$ & $\boldsymbol{G}$ & $d_{g}$ & $v$ & $N$ & $\boldsymbol{G}$ & $d_{g}$ & $\boldsymbol{h}_{g}$ & $v$ & $I c_{G}$ & $I m_{G}$ & $I c_{V}$ & $I m_{V}$ & \\
\hline 5 & & & & & 5000 & & & & 0 & & & & & 5000 & & & & 0 & & & 0.0 & 0.0 & 5 \\
\hline 10 & 1.4 & 1.4 & 2.1 & & 5000 & 0.0 & 0.2 & 1.1 & 0 & & & & & 5000 & 0.0 & 0.2 & 1.1 & 0 & 0.03 & 0.00 & 0.1 & 0.0 & 10 \\
\hline 15 & 4.2 & 4.1 & 5.9 & & 5000 & 1.5 & 2.0 & 3.3 & 5 & & & & & 5000 & 1.5 & 2.0 & 3.3 & 5 & 0.78 & 0.10 & 2.5 & 0.3 & 15 \\
\hline 20 & 6.7 & 6.7 & 9.5 & & 5000 & 7.4 & 4.3 & 5.3 & 31 & & & & & 5000 & 7.4 & 4.3 & 5.4 & 31 & 1.79 & 0.37 & 10.0 & 1.5 & 20 \\
\hline 25 & 8.9 & 8.9 & 14.4 & 19.0 & 5000 & 23.0 & 7.7 & 6.5 & 115 & 993 & 2.3 & 5.5 & 12 & 4007 & 20.7 & 8.1 & 6.6 & 103 & 2.94 & 0.92 & 18.1 & 4.6 & 25 \\
\hline 30 & 11.1 & 11.0 & 18.3 & 19.0 & 4007 & 32.1 & 10.1 & 8.9 & 184 & 1395 & 6.0 & 7.4 & 35 & 2611 & 26.1 & 11.3 & 9.0 & 150 & 1.80 & 1.15 & 14.4 & 6.5 & 30 \\
\hline 35 & 13.1 & 13.0 & 21.5 & 19.0 & 2611 & 34.6 & 13.0 & 11.0 & 221 & 742 & 5.4 & 9.6 & 35 & 1869 & 29.2 & 14.1 & 11.1 & 187 & 1.61 & 1.23 & 14.3 & 7.6 & 35 \\
\hline 40 & 15.0 & 14.8 & 24.6 & 19.0 & 1869 & 36.8 & 15.8 & 13.0 & 257 & 438 & 4.8 & 11.9 & 34 & 1431 & 32.0 & 16.9 & 13.1 & 224 & 1.45 & 1.27 & 13.9 & 8.5 & 40 \\
\hline 45 & 16.7 & 16.5 & 27.6 & 19.0 & 1431 & 38.9 & 18.6 & 14.7 & 292 & 279 & 4.3 & 14.1 & 33 & 1152 & 34.5 & 19.5 & 14.9 & 259 & 1.31 & 1.28 & 13.4 & 9.0 & 45 \\
\hline 50 & 18.2 & 18.1 & 30.3 & 19.0 & 1152 & 40.7 & 21.2 & 16.3 & 325 & 188 & 3.9 & 16.2 & 31 & 963 & 36.8 & 22.1 & 16.5 & 293 & 1.18 & 1.27 & 12.7 & 9.4 & 50 \\
\hline 55 & 19.6 & 19.5 & 32.8 & 19.1 & 963 & 42.5 & 23.7 & 17.8 & 355 & 144 & 3.8 & 18.4 & 32 & 819 & 38.6 & 24.5 & 18.0 & 323 & 1.07 & 1.26 & 12.0 & 9.7 & 55 \\
\hline 60 & 20.9 & 20.7 & 35.2 & 19.3 & 819 & 43.7 & 26.1 & 19.1 & 381 & 105 & 3.5 & 20.5 & 30 & 714 & 40.2 & 26.8 & 19.3 & 351 & 0.96 & 1.24 & 11.2 & 9.9 & 60 \\
\hline 65 & 22.0 & 21.8 & 37.3 & 19.4 & 714 & 44.8 & 28.3 & 20.3 & 405 & 79 & 3.1 & 22.5 & 28 & 635 & 41.7 & 28.9 & 20.5 & 376 & 0.87 & 1.22 & 10.4 & 9.9 & 65 \\
\hline 70 & 23.0 & 22.8 & 39.3 & 19.5 & 635 & 45.8 & 30.3 & 21.3 & 426 & 61 & 2.9 & 24.4 & 27 & 573 & 43.0 & 30.9 & 21.6 & 399 & 0.79 & 1.19 & 9.6 & 9.9 & 70 \\
\hline 75 & 23.9 & 23.7 & 41.1 & 19.6 & 573 & 46.8 & 32.2 & 22.2 & 446 & 49 & 2.6 & 26.2 & 25 & 525 & 44.1 & 32.7 & 22.5 & 421 & 0.73 & 1.16 & 8.9 & 9.9 & 75 \\
\hline 80 & 24.7 & 24.5 & 42.8 & 19.8 & 525 & 47.6 & 34.0 & 23.0 & 463 & 39 & 2.4 & 28.0 & 23 & 486 & 45.2 & 34.4 & 23.3 & 440 & 0.66 & 1.13 & 8.2 & 9.8 & 80 \\
\hline 85 & 25.3 & 25.1 & 44.4 & 19.9 & 486 & 48.4 & 35.6 & 23.7 & 479 & 32 & 2.2 & 29.7 & 22 & 454 & 46.2 & 36.0 & 24.0 & 458 & 0.61 & 1.10 & 7.6 & 9.7 & 85 \\
\hline 90 & 26.0 & 25.7 & 45.8 & 20.0 & 454 & 49.1 & 37.1 & 24.4 & 494 & 26 & 2.0 & 31.3 & 20 & 427 & 47.1 & 37.5 & 24.7 & 474 & 0.57 & 1.07 & 7.0 & 9.6 & 90 \\
\hline 95 & 26.5 & 26.3 & 47.1 & 20.2 & 427 & 49.8 & 38.5 & 24.9 & 507 & 22 & 1.9 & 32.8 & 19 & 405 & 48.0 & 38.8 & 25.2 & 488 & 0.53 & 1.05 & 6.5 & 9.4 & 95 \\
\hline 100 & 27.0 & 26.7 & 48.3 & 20.3 & 405 & 50.5 & 39.8 & 25.4 & 520 & 19 & 1.7 & 34.3 & 18 & 386 & 48.8 & 40.1 & 25.7 & 502 & 0.49 & 1.02 & 6.0 & 9.3 & 100 \\
\hline 105 & 27.4 & 27.1 & 49.4 & 20.4 & 386 & 51.1 & 41.1 & 25.8 & 531 & 16 & 1.6 & 35.8 & 17 & 370 & 49.5 & 41.3 & 26.1 & 514 & 0.46 & 0.99 & 5.6 & 9.1 & 105 \\
\hline 110 & 27.7 & 27.5 & 50.5 & 20.5 & 370 & 51.8 & 42.2 & 26.2 & 541 & 14 & 1.5 & 37.1 & 16 & 356 & 50.2 & 42.4 & 26.5 & 525 & 0.43 & 0.97 & 5.2 & 8.9 & 110 \\
\hline 115 & 28.0 & 27.8 & 51.5 & 20.7 & 356 & 52.3 & 43.3 & 26.6 & 551 & 12 & 1.4 & 38.5 & 15 & 344 & 50.9 & 43.4 & 26.9 & 536 & 0.41 & 0.94 & 4.9 & 8.8 & 115 \\
\hline 120 & 28.3 & 28.1 & 52.4 & 20.8 & 344 & 52.9 & 44.3 & 26.8 & 560 & 11 & 1.3 & 39.8 & 14 & 333 & 51.6 & 44.4 & 27.2 & 545 & 0.39 & 0.92 & 4.6 & 8.6 & 120 \\
\hline
\end{tabular}




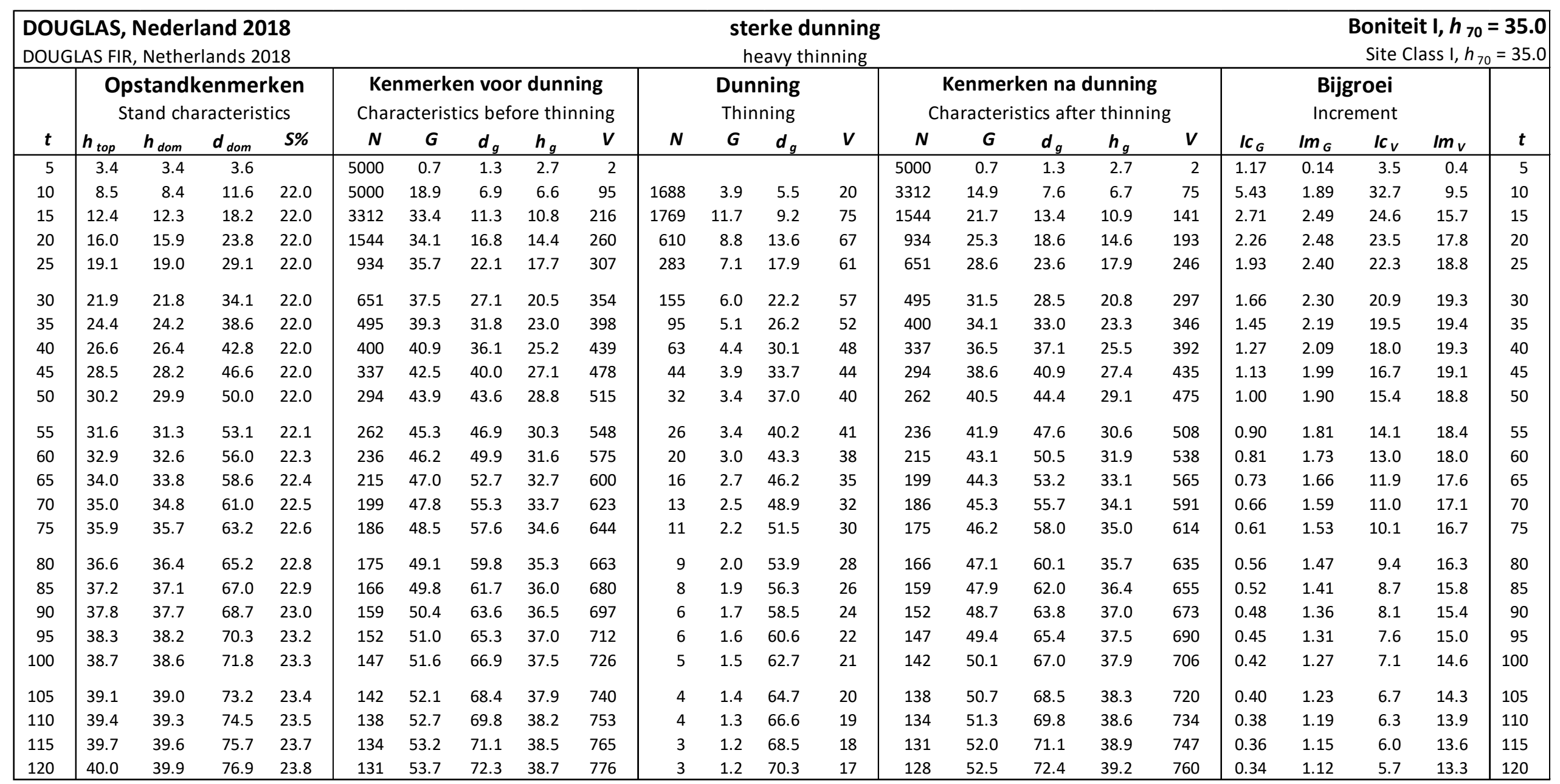




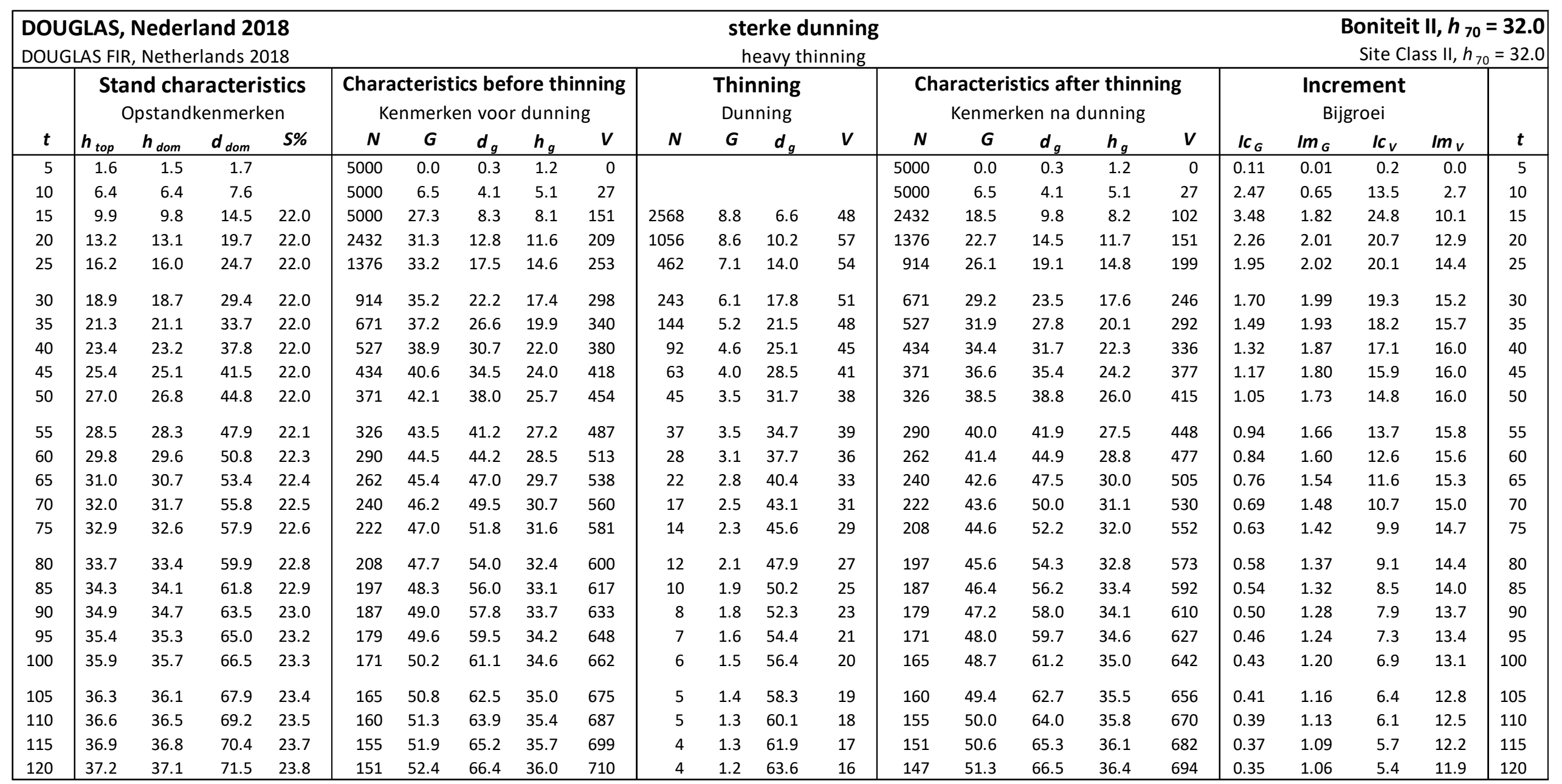




\begin{tabular}{|c|c|c|c|c|c|c|c|c|c|c|c|c|c|c|c|c|c|c|c|c|c|c|c|}
\hline \multicolumn{5}{|c|}{$\begin{array}{l}\text { DOUGLAS, Nederland } 2018 \\
\text { DOUGLAS FIR, Netherlands } 2018\end{array}$} & \multicolumn{15}{|c|}{$\begin{array}{l}\text { sterke dunning } \\
\text { heavy thinning }\end{array}$} & \multicolumn{4}{|c|}{$\begin{array}{r}\text { Boniteit III, } \boldsymbol{h}_{70}=\mathbf{2 9 . 0} \\
\text { Site Class III, } h_{70}=29.0\end{array}$} \\
\hline \multirow[b]{2}{*}{$t$} & \multicolumn{4}{|c|}{$\begin{array}{l}\text { Opstandkenmerken } \\
\text { Stand characteristics }\end{array}$} & \multicolumn{5}{|c|}{$\begin{array}{l}\text { Kenmerken voor dunning } \\
\text { Characteristics before thinning }\end{array}$} & \multicolumn{4}{|c|}{$\begin{array}{l}\text { Dunning } \\
\text { Thinning }\end{array}$} & \multicolumn{5}{|c|}{$\begin{array}{c}\text { Kenmerken na dunning } \\
\text { Characteristics after thinning }\end{array}$} & \multicolumn{4}{|c|}{$\begin{array}{l}\text { Bijgroei } \\
\text { Increment }\end{array}$} & \multirow[b]{2}{*}{$t$} \\
\hline & $h_{\text {top }}$ & $\boldsymbol{h}_{\text {dom }}$ & $\boldsymbol{d}_{d o m}$ & S\% & $N$ & $G$ & $d_{g}$ & $h_{g}$ & $v$ & $N$ & $\boldsymbol{G}$ & $d_{g}$ & $v$ & $N$ & $\boldsymbol{G}$ & $d_{g}$ & $h_{g}$ & $v$ & $I c_{G}$ & $I m_{G}$ & $I c_{v}$ & $I m_{V}$ & \\
\hline 5 & & & & & 5000 & & & & 0 & & & & & 5000 & & & & 0 & & & 0.0 & 0.0 & 5 \\
\hline 10 & 4.4 & 4.4 & 5.2 & & 5000 & 1.9 & 2.2 & 3.5 & 6 & & & & & 5000 & 1.9 & 2.2 & 3.5 & 6 & 1.26 & 0.19 & 4.3 & 0.6 & 10 \\
\hline 15 & 7.8 & 7.7 & 10.7 & 22.0 & 5000 & 14.0 & 6.0 & 5.9 & 66 & 1056 & 1.7 & 4.5 & 8 & 3944 & 12.3 & 6.3 & 5.9 & 58 & 4.30 & 0.94 & 23.1 & 4.4 & 15 \\
\hline 20 & 10.7 & 10.6 & 16.5 & 22.0 & 3944 & 29.3 & 9.7 & 9.0 & 169 & 1863 & 8.4 & 7.6 & 49 & 2082 & 20.9 & 11.3 & 9.1 & 120 & 2.34 & 1.55 & 18.5 & 8.8 & 20 \\
\hline 25 & 13.5 & 13.4 & 21.0 & 22.0 & 2082 & 31.3 & 13.8 & 11.9 & 209 & 767 & 7.1 & 10.8 & 47 & 1315 & 24.2 & 15.3 & 12.0 & 162 & 1.95 & 1.66 & 17.9 & 10.6 & 25 \\
\hline 30 & 16.0 & 15.9 & 25.3 & 22.0 & 1315 & 33.4 & 18.0 & 14.5 & 250 & 386 & 6.1 & 14.2 & 46 & 929 & 27.3 & 19.3 & 14.7 & 205 & 1.71 & 1.68 & 17.4 & 11.8 & 30 \\
\hline 35 & 18.3 & 18.2 & 29.5 & 22.0 & 929 & 35.3 & 22.0 & 16.9 & 290 & 221 & 5.3 & 17.5 & 44 & 709 & 30.0 & 23.2 & 17.1 & 247 & 1.52 & 1.67 & 16.8 & 12.6 & 35 \\
\hline 40 & 20.4 & 20.3 & 33.3 & 22.0 & 709 & 37.2 & 25.8 & 19.0 & 328 & 138 & 4.7 & 20.7 & 41 & 571 & 32.5 & 26.9 & 19.2 & 287 & 1.35 & 1.64 & 15.9 & 13.0 & 40 \\
\hline 45 & 22.3 & 22.1 & 36.9 & 22.0 & 571 & 38.9 & 29.5 & 20.9 & 364 & 92 & 4.1 & 23.9 & 39 & 478 & 34.8 & 30.4 & 21.2 & 326 & 1.20 & 1.60 & 15.0 & 13.3 & 45 \\
\hline 50 & 24.0 & 23.8 & 40.1 & 22.0 & 478 & 40.4 & 32.8 & 22.6 & 398 & 65 & 3.6 & 26.8 & 36 & 414 & 36.8 & 33.6 & 22.9 & 362 & 1.08 & 1.56 & 14.0 & 13.4 & 50 \\
\hline 55 & 25.5 & 25.3 & 43.1 & 22.1 & 414 & 41.9 & 35.9 & 24.1 & 429 & 51 & 3.5 & 29.7 & 36 & 363 & 38.3 & 36.7 & 24.4 & 393 & 0.97 & 1.51 & 13.0 & 13.4 & 55 \\
\hline 60 & 26.8 & 26.6 & 45.9 & 22.3 & 363 & 42.9 & 38.8 & 25.5 & 456 & 39 & 3.2 & 32.5 & 34 & 324 & 39.7 & 39.5 & 25.8 & 422 & 0.87 & 1.46 & 12.0 & 13.3 & 60 \\
\hline 65 & 28.0 & 27.7 & 48.5 & 22.4 & 324 & 43.9 & 41.5 & 26.7 & 479 & 30 & 2.9 & 35.1 & 31 & 294 & 41.0 & 42.1 & 27.0 & 448 & 0.79 & 1.41 & 11.1 & 13.2 & 65 \\
\hline 70 & 29.0 & 28.7 & 50.8 & 22.5 & 294 & 44.7 & 44.0 & 27.7 & 501 & 24 & 2.6 & 37.6 & 29 & 271 & 42.1 & 44.5 & 28.0 & 472 & 0.71 & 1.36 & 10.3 & 13.0 & 70 \\
\hline 75 & 29.9 & 29.6 & 52.9 & 22.6 & 271 & 45.5 & 46.3 & 28.6 & 521 & 19 & 2.4 & 40.0 & 27 & 252 & 43.2 & 46.7 & 28.9 & 494 & 0.65 & 1.32 & 9.5 & 12.8 & 75 \\
\hline 80 & 30.7 & 30.4 & 54.9 & 22.8 & 252 & 46.3 & 48.4 & 29.4 & 540 & 15 & 2.2 & 42.2 & 25 & 236 & 44.1 & 48.7 & 29.8 & 514 & 0.60 & 1.27 & 8.8 & 12.6 & 80 \\
\hline 85 & 31.4 & 31.1 & 56.7 & 22.9 & 236 & 47.0 & 50.3 & 30.1 & 556 & 13 & 2.0 & 44.4 & 24 & 224 & 45.0 & 50.6 & 30.5 & 533 & 0.55 & 1.23 & 8.1 & 12.3 & 85 \\
\hline 90 & 32.0 & 31.8 & 58.4 & 23.0 & 224 & 47.7 & 52.1 & 30.7 & 572 & 11 & 1.8 & 46.4 & 22 & 213 & 45.8 & 52.4 & 31.1 & 550 & 0.51 & 1.19 & 7.5 & 12.1 & 90 \\
\hline 95 & 32.5 & 32.3 & 59.9 & 23.2 & 213 & 48.3 & 53.8 & 31.3 & 586 & 9 & 1.7 & 48.4 & 20 & 204 & 46.6 & 54.0 & 31.6 & 566 & 0.48 & 1.16 & 7.0 & 11.8 & 95 \\
\hline 100 & 33.0 & 32.8 & 61.3 & 23.3 & 204 & 48.9 & 55.3 & 31.7 & 600 & 8 & 1.6 & 50.2 & 19 & 196 & 47.3 & 55.5 & 32.1 & 580 & 0.44 & 1.12 & 6.5 & 11.6 & 100 \\
\hline 105 & 33.4 & 33.2 & 62.7 & 23.4 & 196 & 49.5 & 56.7 & 32.2 & 612 & 7 & 1.4 & 52.1 & 18 & 189 & 48.1 & 56.9 & 32.5 & 594 & 0.42 & 1.09 & 6.1 & 11.3 & 105 \\
\hline 110 & 33.7 & 33.6 & 63.9 & 23.5 & 189 & 50.1 & 58.1 & 32.5 & 624 & 6 & 1.4 & 53.8 & 17 & 183 & 48.7 & 58.2 & 32.9 & 607 & 0.39 & 1.06 & 5.7 & 11.1 & 110 \\
\hline 115 & 34.0 & 33.9 & 65.1 & 23.7 & 183 & 50.7 & 59.4 & 32.8 & 635 & 5 & 1.3 & 55.5 & 16 & 178 & 49.4 & 59.5 & 33.2 & 619 & 0.37 & 1.03 & 5.4 & 10.8 & 115 \\
\hline 120 & 34.3 & 34.2 & 66.2 & 23.8 & 178 & 51.2 & 60.6 & 33.1 & 645 & 5 & 1.2 & 57.1 & 15 & 173 & 50.0 & 60.7 & 33.5 & 630 & 0.36 & 1.00 & 5.1 & 10.6 & 120 \\
\hline
\end{tabular}




\begin{tabular}{|c|c|c|c|c|c|c|c|c|c|c|c|c|c|c|c|c|c|c|c|c|c|c|c|}
\hline \multicolumn{5}{|c|}{$\begin{array}{l}\text { DOUGLAS, Nederland } 2018 \\
\text { DOUGLAS FIR, Netherlands } 2018\end{array}$} & \multicolumn{15}{|c|}{$\begin{array}{l}\text { sterke dunning } \\
\text { heavy thinning }\end{array}$} & \multicolumn{4}{|c|}{$\begin{array}{r}\text { Boniteit IV, } \boldsymbol{h}_{70}=\mathbf{2 6 . 0} \\
\text { Site Class IV, } h_{70}=26.0\end{array}$} \\
\hline \multirow[b]{2}{*}{$t$} & \multicolumn{4}{|c|}{$\begin{array}{l}\text { Opstandkenmerken } \\
\text { Stand characteristics }\end{array}$} & \multicolumn{5}{|c|}{$\begin{array}{l}\text { Kenmerken voor dunning } \\
\text { Characteristics before thinning }\end{array}$} & \multicolumn{4}{|c|}{$\begin{array}{l}\text { Dunning } \\
\text { Thinning }\end{array}$} & \multicolumn{5}{|c|}{$\begin{array}{c}\text { Kenmerken na dunning } \\
\text { Characteristics after thinning }\end{array}$} & \multicolumn{4}{|c|}{$\begin{array}{l}\text { Bijgroei } \\
\text { Increment }\end{array}$} & \multirow[b]{2}{*}{$t$} \\
\hline & $h_{\text {top }}$ & $\boldsymbol{h}_{\text {dom }}$ & $\boldsymbol{d}_{d o m}$ & S\% & $N$ & $\boldsymbol{G}$ & $d_{g}$ & $h_{g}$ & $v$ & $N$ & $\boldsymbol{G}$ & $d_{g}$ & $v$ & $N$ & $\boldsymbol{G}$ & $d_{g}$ & $h_{g}$ & $v$ & $I c_{G}$ & $I m_{G}$ & $I c_{v}$ & $I m_{V}$ & \\
\hline 5 & & & & & 5000 & & & & 0 & & & & & 5000 & & & & 0 & & & 0.0 & 0.0 & 5 \\
\hline 10 & 2.7 & 2.6 & 3.1 & & 5000 & 0.3 & 0.8 & 2.1 & 1 & & & & & 5000 & 0.3 & 0.8 & 2.1 & 1 & 0.28 & 0.03 & 0.7 & 0.1 & 10 \\
\hline 15 & 5.9 & 5.9 & 7.6 & & 5000 & 5.2 & 3.6 & 4.7 & 20 & & & & & 5000 & 5.2 & 3.6 & 4.7 & 20 & 1.51 & 0.35 & 6.4 & 1.3 & 15 \\
\hline 20 & 8.6 & 8.5 & 12.9 & 22.0 & 5000 & 19.5 & 7.1 & 6.7 & 96 & 1751 & 3.9 & 5.3 & 19 & 3249 & 15.6 & 7.8 & 6.8 & 77 & 3.35 & 0.98 & 19.8 & 4.8 & 20 \\
\hline 25 & 11.1 & 11.0 & 17.7 & 22.0 & 3249 & 28.6 & 10.6 & 9.4 & 166 & 1301 & 6.8 & 8.1 & 39 & 1948 & 21.9 & 12.0 & 9.5 & 127 & 1.92 & 1.30 & 15.4 & 7.4 & 25 \\
\hline 30 & 13.4 & 13.3 & 21.7 & 22.0 & 1948 & 30.9 & 14.2 & 11.8 & 204 & 625 & 5.9 & 11.0 & 39 & 1323 & 25.0 & 15.5 & 12.0 & 165 & 1.70 & 1.39 & 15.4 & 8.8 & 30 \\
\hline 35 & 15.6 & 15.5 & 25.5 & 22.0 & 1323 & 33.0 & 17.8 & 14.1 & 241 & 345 & 5.2 & 13.9 & 38 & 978 & 27.8 & 19.0 & 14.3 & 203 & 1.52 & 1.42 & 15.0 & 9.7 & 35 \\
\hline 40 & 17.6 & 17.5 & 29.1 & 22.0 & 978 & 35.0 & 21.3 & 16.2 & 276 & 210 & 4.6 & 16.8 & 37 & 769 & 30.4 & 22.4 & 16.3 & 240 & 1.36 & 1.42 & 14.4 & 10.3 & 40 \\
\hline 45 & 19.4 & 19.3 & 32.5 & 22.0 & 769 & 36.8 & 24.7 & 18.0 & 310 & 137 & 4.1 & 19.6 & 35 & 632 & 32.7 & 25.7 & 18.2 & 275 & 1.22 & 1.41 & 13.7 & 10.7 & 45 \\
\hline 50 & 21.1 & 20.9 & 35.6 & 22.0 & 632 & 38.4 & 27.8 & 19.7 & 342 & 94 & 3.7 & 22.3 & 33 & 538 & 34.7 & 28.7 & 19.9 & 309 & 1.09 & 1.38 & 12.9 & 11.0 & 50 \\
\hline 55 & 22.5 & 22.3 & 38.5 & 22.1 & 538 & 39.9 & 30.8 & 21.2 & 372 & 73 & 3.6 & 25.0 & 33 & 465 & 36.4 & 31.6 & 21.4 & 339 & 0.98 & 1.35 & 12.1 & 11.1 & 55 \\
\hline 60 & 23.8 & 23.6 & 41.2 & 22.3 & 465 & 41.0 & 33.5 & 22.5 & 397 & 54 & 3.2 & 27.5 & 31 & 411 & 37.8 & 34.2 & 22.7 & 366 & 0.89 & 1.31 & 11.2 & 11.2 & 60 \\
\hline 65 & 25.0 & 24.8 & 43.6 & 22.4 & 411 & 42.1 & 36.1 & 23.7 & 420 & 41 & 2.9 & 30.0 & 29 & 369 & 39.1 & 36.7 & 23.9 & 391 & 0.80 & 1.28 & 10.4 & 11.1 & 65 \\
\hline 70 & 26.0 & 25.8 & 45.9 & 22.5 & 369 & 43.0 & 38.5 & 24.7 & 441 & 32 & 2.6 & 32.3 & 27 & 337 & 40.3 & 39.0 & 25.0 & 414 & 0.73 & 1.24 & 9.6 & 11.1 & 70 \\
\hline 75 & 26.9 & 26.7 & 48.0 & 22.6 & 337 & 43.8 & 40.7 & 25.6 & 460 & 26 & 2.4 & 34.5 & 25 & 311 & 41.4 & 41.2 & 25.9 & 435 & 0.67 & 1.21 & 8.9 & 10.9 & 75 \\
\hline 80 & 27.7 & 27.5 & 49.9 & 22.8 & 311 & 44.6 & 42.7 & 26.4 & 478 & 21 & 2.2 & 36.6 & 24 & 290 & 42.4 & 43.1 & 26.7 & 454 & 0.61 & 1.17 & 8.2 & 10.8 & 80 \\
\hline 85 & 28.4 & 28.1 & 51.6 & 22.9 & 290 & 45.4 & 44.6 & 27.1 & 494 & 17 & 2.0 & 38.6 & 22 & 273 & 43.3 & 44.9 & 27.4 & 472 & 0.56 & 1.14 & 7.6 & 10.6 & 85 \\
\hline 90 & 29.0 & 28.7 & 53.2 & 23.0 & 273 & 46.1 & 46.3 & 27.8 & 509 & 14 & 1.9 & 40.6 & 20 & 259 & 44.2 & 46.6 & 28.1 & 488 & 0.52 & 1.10 & 7.1 & 10.4 & 90 \\
\hline 95 & 29.5 & 29.3 & 54.7 & 23.2 & 259 & 46.7 & 47.9 & 28.3 & 522 & 12 & 1.7 & 42.4 & 19 & 247 & 45.0 & 48.2 & 28.6 & 503 & 0.49 & 1.07 & 6.6 & 10.3 & 95 \\
\hline 100 & 30.0 & 29.7 & 56.1 & 23.3 & 247 & 47.4 & 49.4 & 28.8 & 535 & 10 & 1.6 & 44.2 & 18 & 237 & 45.8 & 49.6 & 29.1 & 517 & 0.45 & 1.04 & 6.1 & 10.1 & 100 \\
\hline 105 & 30.4 & 30.2 & 57.4 & 23.4 & 237 & 48.0 & 50.8 & 29.2 & 547 & 9 & 1.5 & 45.9 & 17 & 228 & 46.5 & 51.0 & 29.5 & 530 & 0.43 & 1.01 & 5.7 & 9.9 & 105 \\
\hline 110 & 30.8 & 30.5 & 58.6 & 23.5 & 228 & 48.6 & 52.1 & 29.6 & 557 & 8 & 1.4 & 47.5 & 16 & 220 & 47.2 & 52.3 & 29.9 & 542 & 0.40 & 0.99 & 5.3 & 9.7 & 110 \\
\hline 115 & 31.1 & 30.9 & 59.7 & 23.7 & 220 & 49.2 & 53.3 & 29.9 & 568 & 7 & 1.3 & 49.1 & 15 & 213 & 47.9 & 53.5 & 30.2 & 553 & 0.38 & 0.96 & 5.0 & 9.5 & 115 \\
\hline 120 & 31.4 & 31.2 & 60.8 & 23.8 & 213 & 49.8 & 54.5 & 30.2 & 577 & 6 & 1.2 & 50.6 & 14 & 207 & 48.6 & 54.6 & 30.5 & 563 & 0.36 & 0.94 & 4.7 & 9.3 & 120 \\
\hline
\end{tabular}




\begin{tabular}{|c|c|c|c|c|c|c|c|c|c|c|c|c|c|c|c|c|c|c|c|c|c|c|c|}
\hline \multicolumn{5}{|c|}{$\begin{array}{l}\text { DOUGLAS, Nederland } 2018 \\
\text { DOUGLAS FIR, Netherlands } 2018\end{array}$} & \multicolumn{15}{|c|}{$\begin{array}{c}\text { sterke dunning } \\
\text { heavy thinning }\end{array}$} & \multicolumn{4}{|c|}{$\begin{array}{r}\text { Boniteit V, } \boldsymbol{h}_{70}=\mathbf{2 3 . 0} \\
\text { Site Class } \mathbf{V}, h_{70}=23.0\end{array}$} \\
\hline \multirow[b]{2}{*}{$t$} & \multicolumn{4}{|c|}{$\begin{array}{l}\text { Opstandkenmerken } \\
\text { Stand characteristics }\end{array}$} & \multicolumn{5}{|c|}{$\begin{array}{l}\text { Kenmerken voor dunning } \\
\text { Characteristics before thinning }\end{array}$} & \multicolumn{4}{|c|}{$\begin{array}{l}\text { Dunning } \\
\text { Thinning }\end{array}$} & \multicolumn{5}{|c|}{$\begin{array}{l}\text { Kenmerken na dunning } \\
\text { Characteristics after thinning }\end{array}$} & \multicolumn{4}{|c|}{$\begin{array}{l}\text { Bijgroei } \\
\text { Increment }\end{array}$} & \multirow[b]{2}{*}{$t$} \\
\hline & $h_{\text {top }}$ & $\boldsymbol{h}_{\text {dom }}$ & $\boldsymbol{d}_{d o m}$ & S\% & $N$ & $\boldsymbol{G}$ & $d_{g}$ & $\boldsymbol{h}_{g}$ & $v$ & $N$ & $\boldsymbol{G}$ & $d_{g}$ & $v$ & $N$ & $\boldsymbol{G}$ & $d_{g}$ & $h_{g}$ & $v$ & $I c_{G}$ & $I m_{G}$ & $I c_{V}$ & $I m_{V}$ & \\
\hline 5 & & & & & 5000 & & & & 0 & & & & & 5000 & & & & 0 & & & 0.0 & 0.0 & 5 \\
\hline 10 & 1.4 & 1.4 & 1.9 & & 5000 & 0.0 & 0.2 & 1.1 & 0 & & & & & 5000 & 0.0 & 0.2 & 1.1 & 0 & 0.03 & 0.00 & 0.1 & 0.0 & 10 \\
\hline 15 & 4.2 & 4.1 & 5.4 & & 5000 & 1.5 & 2.0 & 3.3 & 5 & & & & & 5000 & 1.5 & 2.0 & 3.3 & 5 & 0.78 & 0.10 & 2.5 & 0.3 & 15 \\
\hline 20 & 6.7 & 6.7 & 8.9 & & 5000 & 7.4 & 4.3 & 5.3 & 31 & & & & & 5000 & 7.4 & 4.3 & 5.4 & 31 & 1.74 & 0.37 & 9.8 & 1.5 & 20 \\
\hline 25 & 8.9 & 8.9 & 14.2 & 22.0 & 5000 & 22.0 & 7.5 & 7.1 & 110 & 2012 & 5.0 & 5.6 & 25 & 2988 & 17.0 & 8.5 & 7.2 & 85 & 2.74 & 0.88 & 16.8 & 4.4 & 25 \\
\hline 30 & $\mid 11.1$ & 11.0 & 18.3 & 22.0 & 2988 & 27.7 & 10.9 & 9.4 & 159 & 1041 & 5.5 & 8.2 & 32 & 1948 & 22.2 & 12.0 & 9.5 & 127 & 1.67 & 1.09 & 13.1 & 6.1 & 30 \\
\hline 35 & $\mid 13.1$ & 13.0 & 21.8 & 22.0 & 1948 & 30.1 & 14.0 & 11.5 & 193 & 554 & 5.0 & 10.7 & 32 & 1394 & 25.1 & 15.1 & 11.6 & 161 & 1.50 & 1.16 & 13.1 & 7.1 & 35 \\
\hline 40 & 15.0 & 14.8 & 25.2 & 22.0 & 1394 & 32.2 & 17.2 & 13.4 & 225 & 327 & 4.5 & 13.2 & 31 & 1067 & 27.8 & 18.2 & 13.6 & 194 & 1.35 & 1.19 & 12.8 & 7.8 & 40 \\
\hline 45 & 16.7 & 16.5 & 28.3 & 22.0 & 1067 & 34.2 & 20.2 & 15.2 & 257 & 208 & 4.0 & 15.7 & 30 & 859 & 30.1 & 21.1 & 15.4 & 226 & 1.22 & 1.20 & 12.3 & 8.4 & 45 \\
\hline 50 & 18.2 & 18.1 & 31.3 & 22.0 & 859 & 35.9 & 23.1 & 16.8 & 286 & 140 & 3.6 & 18.1 & 29 & 719 & 32.3 & 23.9 & 17.0 & 257 & 1.10 & 1.20 & 11.7 & 8.7 & 50 \\
\hline 55 & 19.6 & 19.5 & 34.0 & 22.1 & 719 & 37.5 & 25.8 & 18.2 & 314 & 106 & 3.5 & 20.5 & 29 & 612 & 34.0 & 26.6 & 18.4 & 285 & 0.99 & 1.18 & 11.0 & 9.0 & 55 \\
\hline 60 & 20.9 & 20.7 & 36.6 & 22.3 & 612 & 38.7 & 28.4 & 19.5 & 338 & 78 & 3.2 & 22.8 & 28 & 535 & 35.6 & 29.1 & 19.7 & 310 & 0.90 & 1.16 & 10.3 & 9.1 & 60 \\
\hline 65 & 22.0 & 21.8 & 38.9 & 22.4 & 535 & 39.8 & 30.8 & 20.7 & 360 & 59 & 2.9 & 25.1 & 26 & 476 & 36.9 & 31.4 & 20.9 & 333 & 0.81 & 1.14 & 9.6 & 9.2 & 65 \\
\hline 70 & 23.0 & 22.8 & 41.1 & 22.5 & 476 & 40.8 & 33.0 & 21.7 & 379 & 45 & 2.6 & 27.2 & 24 & 431 & 38.2 & 33.6 & 21.9 & 355 & 0.74 & 1.11 & 8.9 & 9.2 & 70 \\
\hline 75 & 23.9 & 23.7 & 43.0 & 22.6 & 431 & 41.7 & 35.1 & 22.6 & 398 & 36 & 2.4 & 29.2 & 23 & 395 & 39.3 & 35.6 & 22.8 & 375 & 0.68 & 1.09 & 8.2 & 9.1 & 75 \\
\hline 80 & 24.7 & 24.5 & 44.9 & 22.8 & 395 & 42.6 & 37.1 & 23.4 & 414 & 29 & 2.2 & 31.2 & 21 & 366 & 40.4 & 37.5 & 23.7 & 393 & 0.62 & 1.06 & 7.6 & 9.0 & 80 \\
\hline 85 & 25.3 & 25.1 & 46.5 & 22.9 & 366 & 43.4 & 38.8 & 24.1 & 429 & 23 & 2.0 & 33.1 & 20 & 343 & 41.3 & 39.2 & 24.4 & 409 & 0.57 & 1.03 & 7.0 & 8.9 & 85 \\
\hline 90 & 26.0 & 25.7 & 48.1 & 23.0 & 343 & 44.1 & 40.5 & 24.7 & 443 & 19 & 1.9 & 34.8 & 19 & 323 & 42.3 & 40.8 & 25.0 & 425 & 0.53 & 1.01 & 6.5 & 8.8 & 90 \\
\hline 95 & 26.5 & 26.3 & 49.5 & 23.2 & 323 & 44.8 & 42.0 & 25.2 & 456 & 16 & 1.7 & 36.5 & 17 & 307 & 43.1 & 42.3 & 25.5 & 439 & 0.49 & 0.98 & 6.1 & 8.7 & 95 \\
\hline 100 & 27.0 & 26.7 & 50.8 & 23.3 & 307 & 45.5 & 43.4 & 25.7 & 468 & 14 & 1.6 & 38.2 & 16 & 293 & 43.9 & 43.7 & 26.0 & 452 & 0.46 & 0.96 & 5.6 & 8.5 & 100 \\
\hline 105 & 27.4 & 27.1 & 52.1 & 23.4 & 293 & 46.1 & 44.8 & 26.1 & 479 & 12 & 1.5 & 39.7 & 15 & 281 & 44.7 & 45.0 & 26.5 & 464 & 0.43 & 0.93 & 5.2 & 8.4 & 105 \\
\hline 110 & 27.7 & 27.5 & 53.2 & 23.5 & 281 & 46.8 & 46.0 & 26.5 & 489 & 10 & 1.4 & 41.3 & 14 & 271 & 45.4 & 46.2 & 26.8 & 475 & 0.41 & 0.91 & 4.9 & 8.2 & 110 \\
\hline 115 & 28.0 & 27.8 & 54.3 & 23.7 & 271 & 47.4 & 47.2 & 26.8 & 498 & 9 & 1.3 & 42.7 & 13 & 262 & 46.1 & 47.3 & 27.2 & 485 & 0.39 & 0.89 & 4.6 & 8.1 & 115 \\
\hline 120 & 28.3 & 28.1 & 55.3 & 23.8 & 262 & 48.0 & 48.3 & 27.1 & 507 & 8 & 1.2 & 44.1 & 13 & 254 & 46.8 & 48.4 & 27.4 & 495 & 0.37 & 0.86 & 4.3 & 7.9 & 120 \\
\hline
\end{tabular}




\section{Japanse lariks (Larix kaempferi)}

Japanese larch

Jansen, J.J., A. Oosterbaan, G.M. J. Mohren en J. den Ouden

Bron: Jansen, J.J., A. Oosterbaan, G.M. J. Mohren en J. den Ouden,

2018. Groei en productie van Japanse lariks in Nederland. FEM Groei en Productie Rapport 2018 - 1. 120 blz.

Dit rapport is gratis te downloaden op:

https://doi.org/10.18174/444088

Er is een tabel voor Noord-Nederland en een tabel voor Zuid-Nederland.

Het bosgebied Zuid-Nederland bestaat uit Noord-Brabant, Noord en Midden Limburg.

Het bosgebied Noord-Nederland bestaat uit de rest van Nederland dus inclusief Zuid-Limburg.

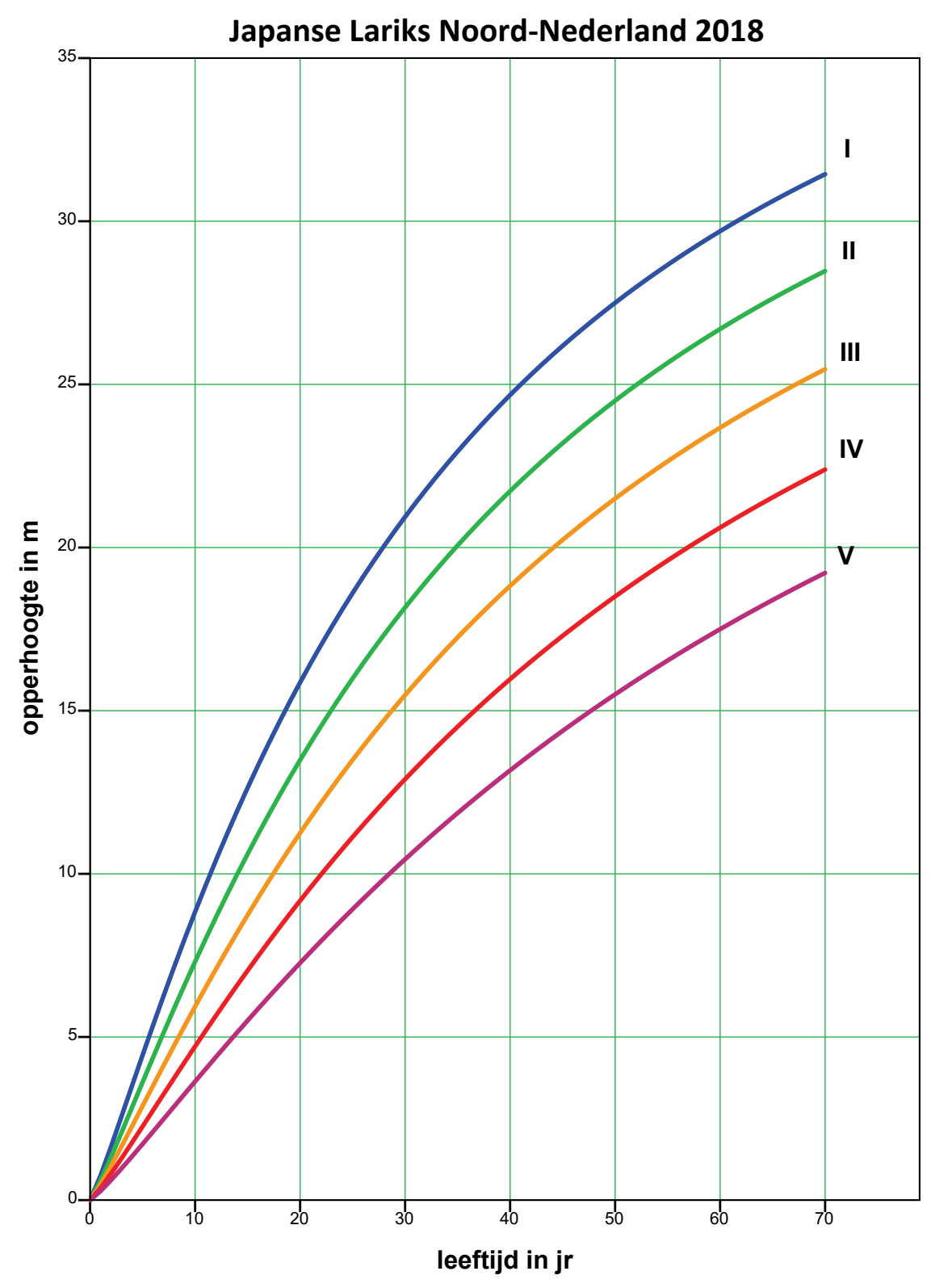




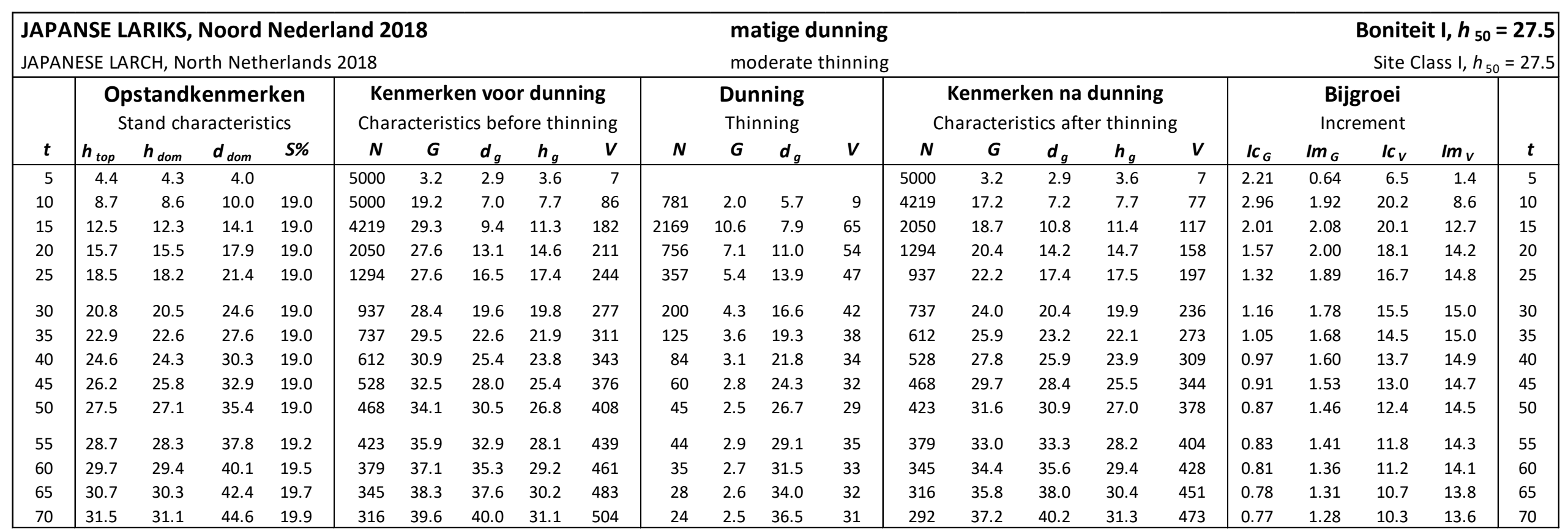




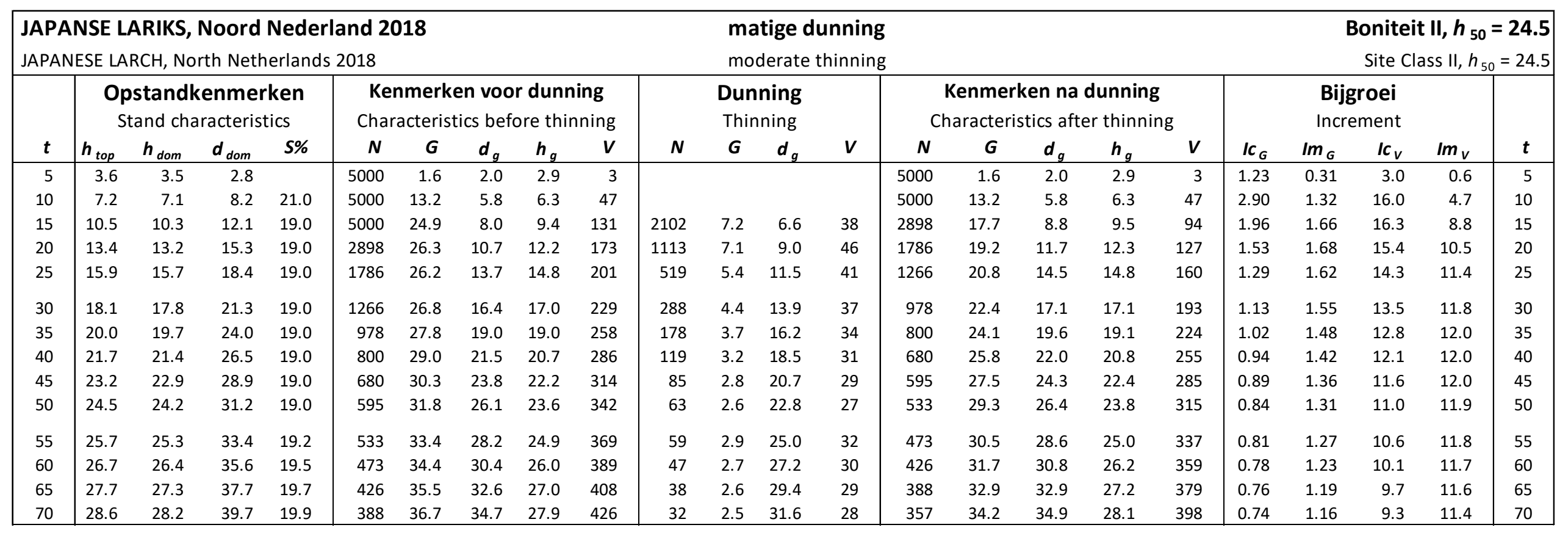




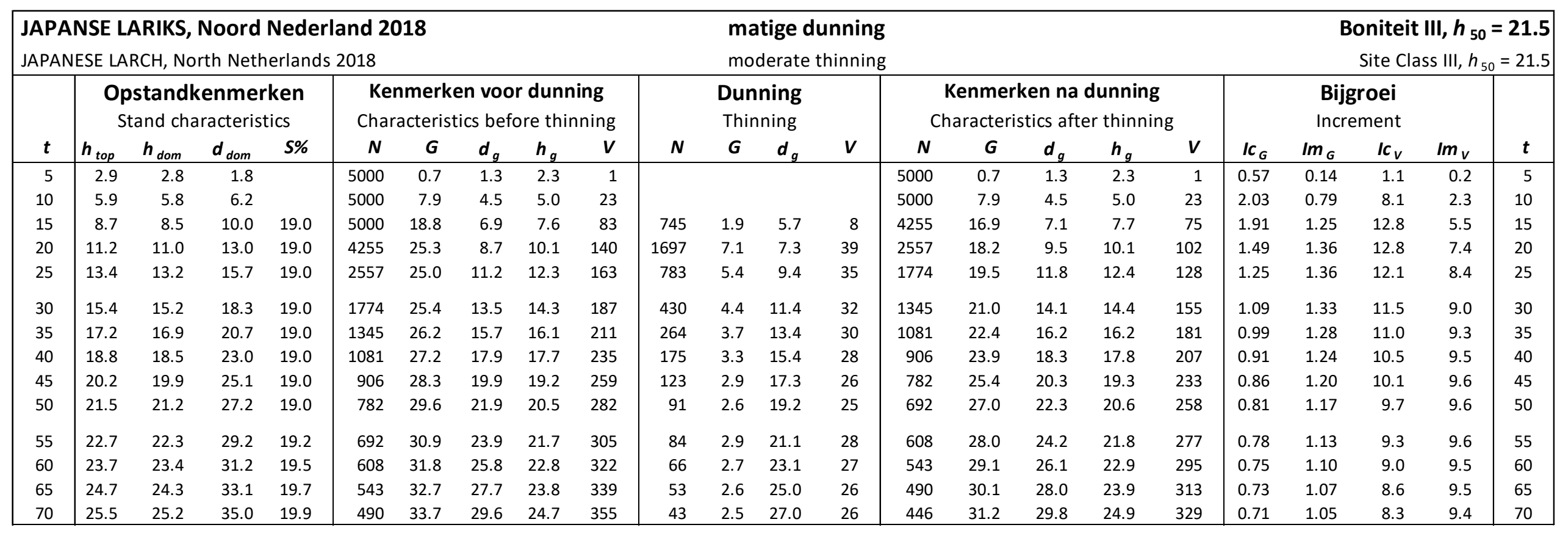




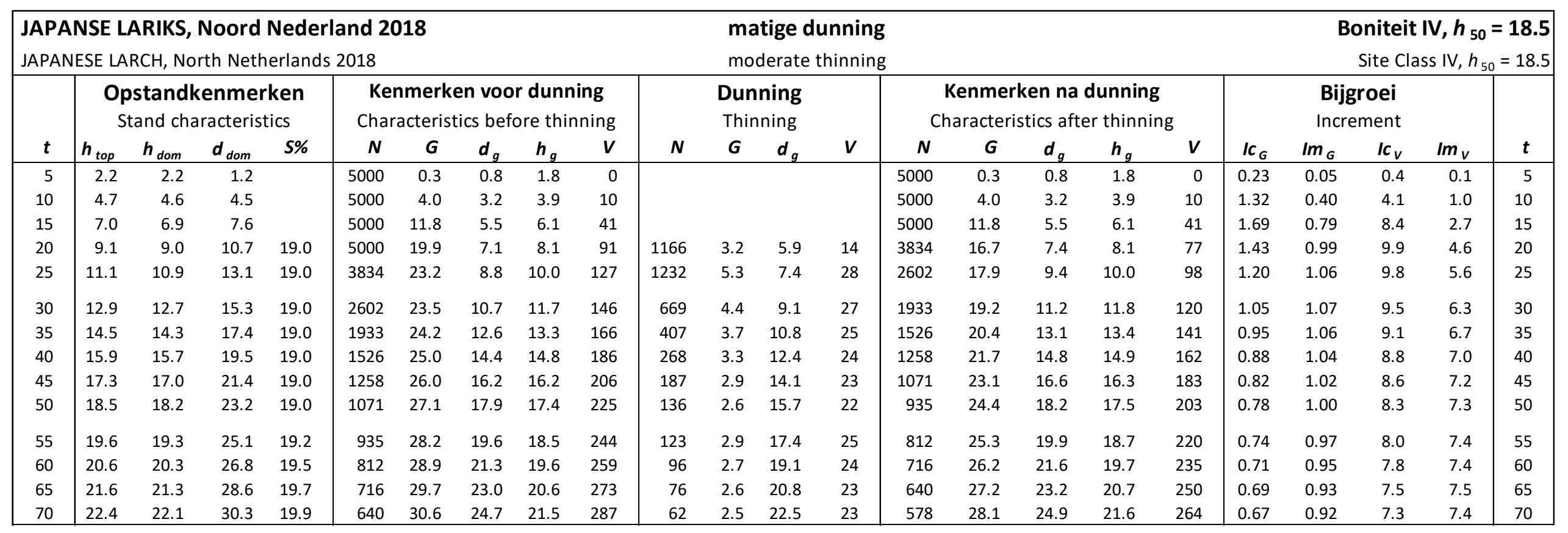




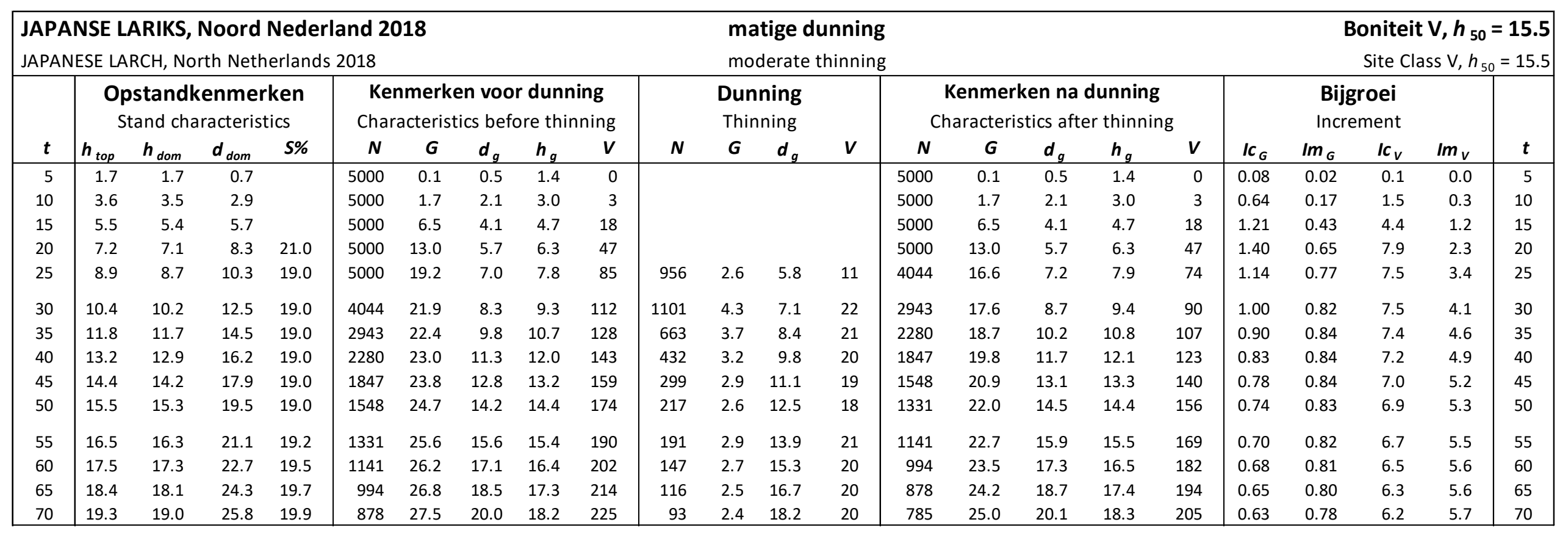




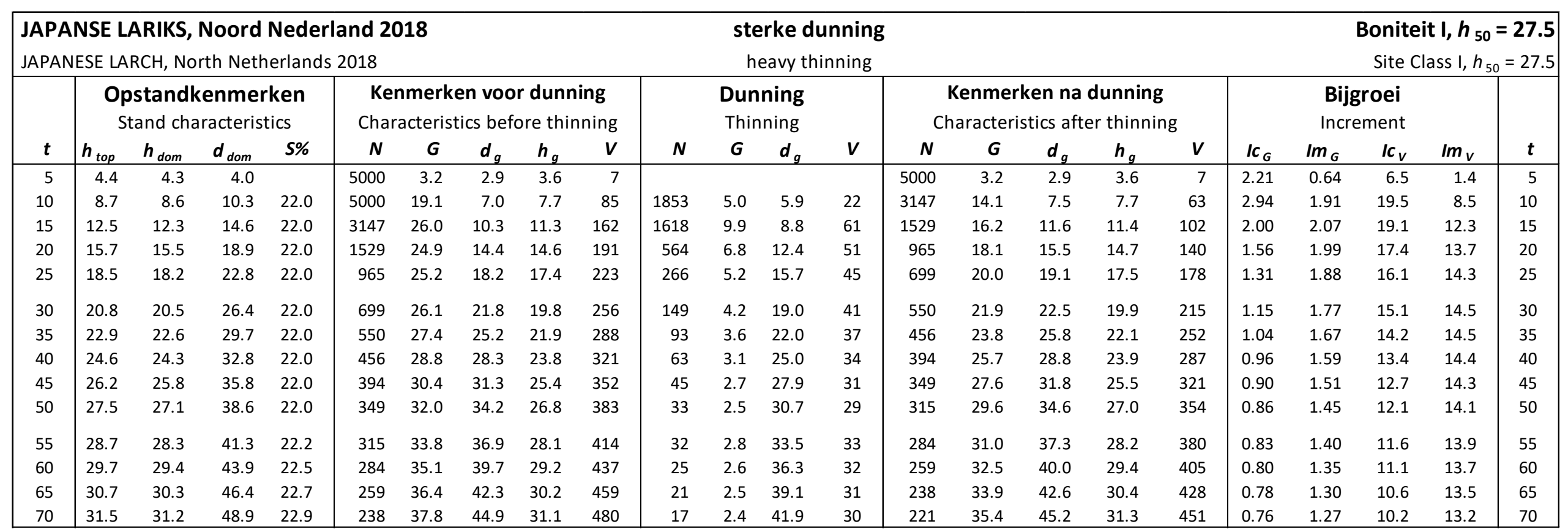




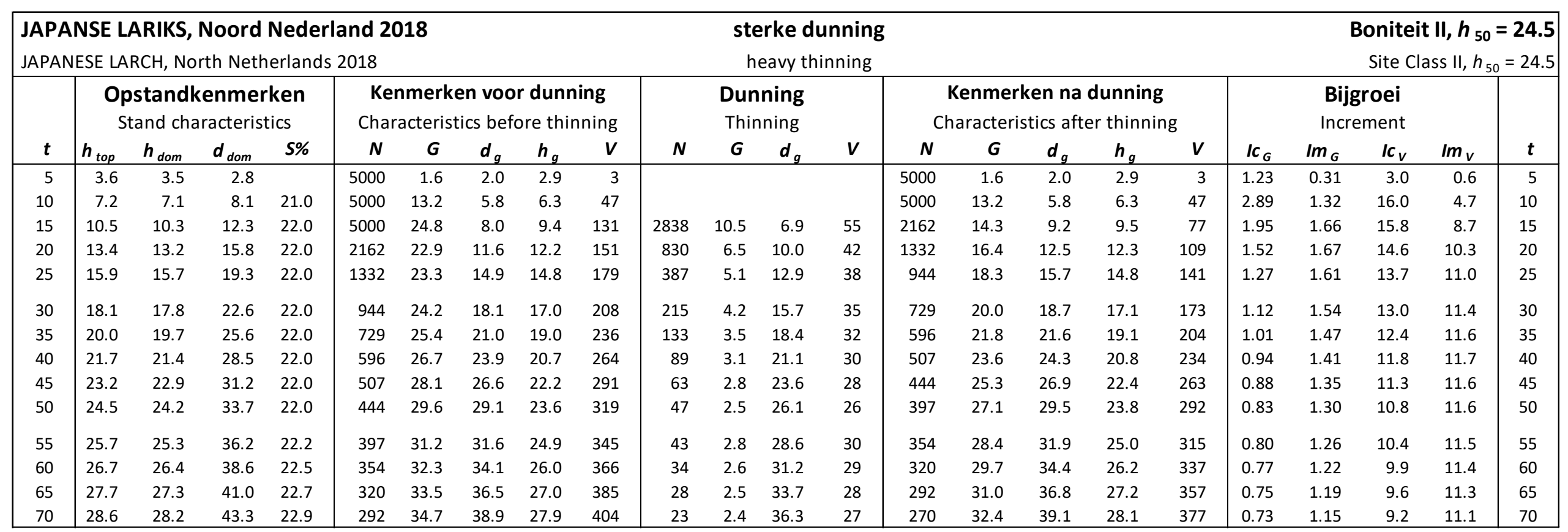




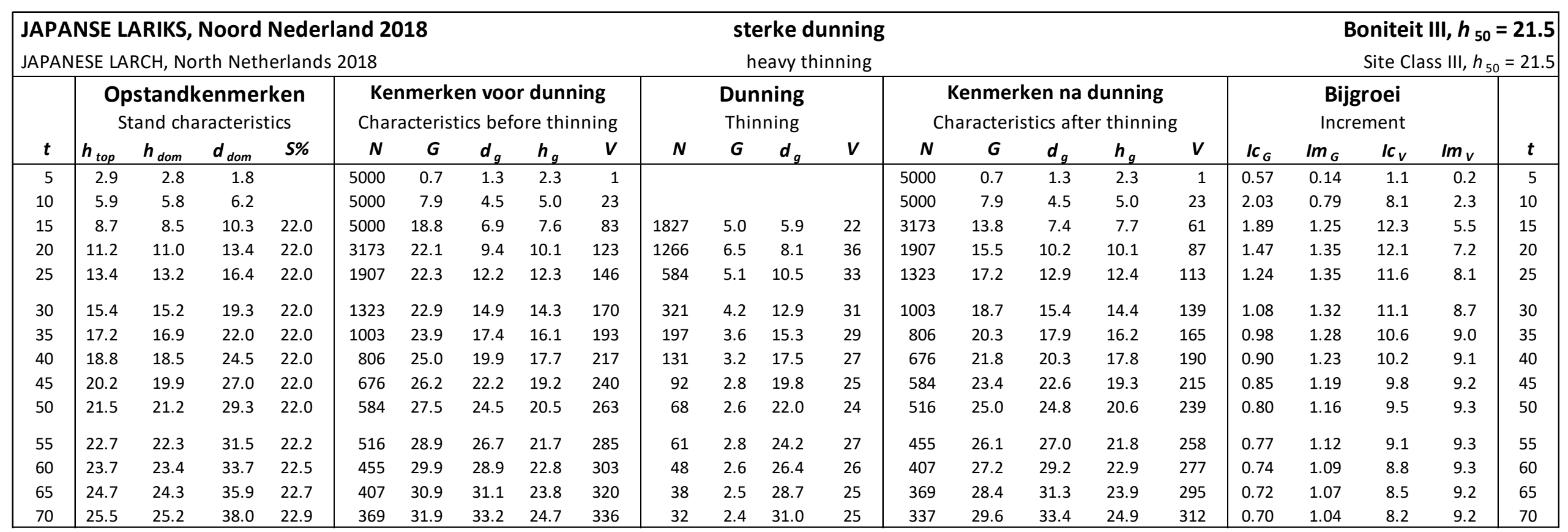




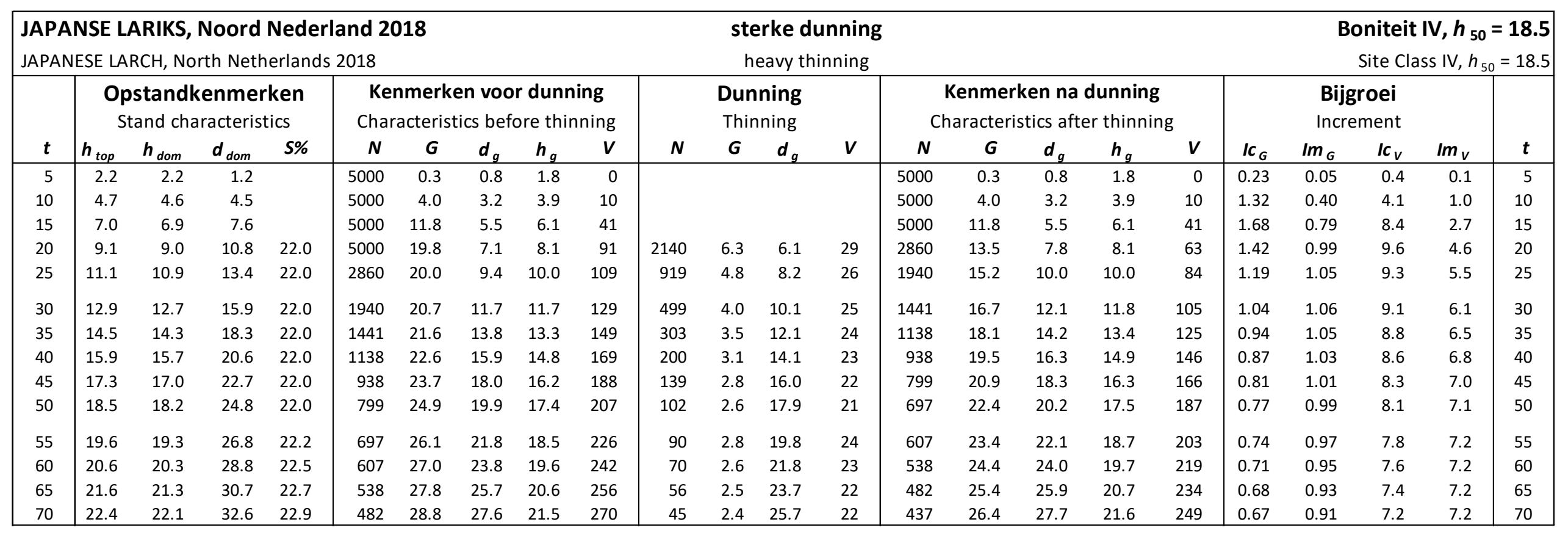




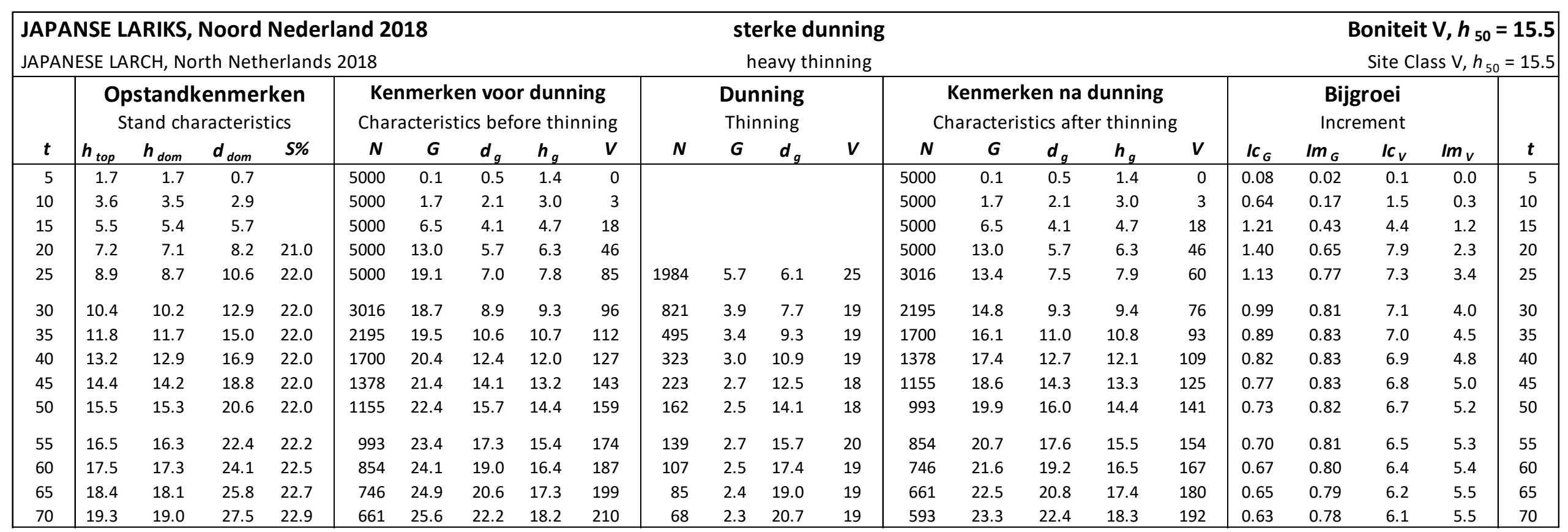


Het bosgebied Zuid-Nederland bestaat uit:

- Noord-Brabant

- Noord en Midden Limburg

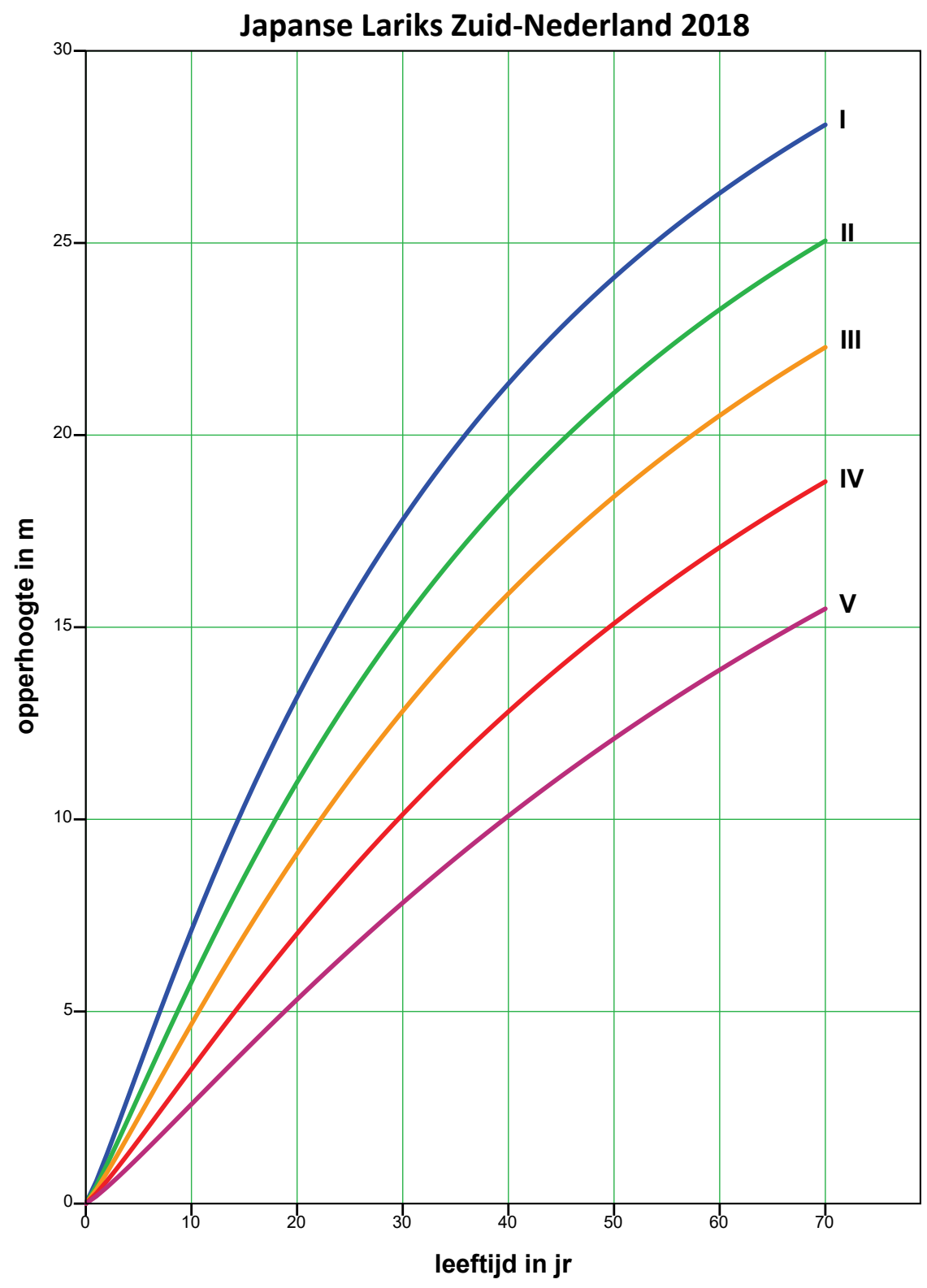




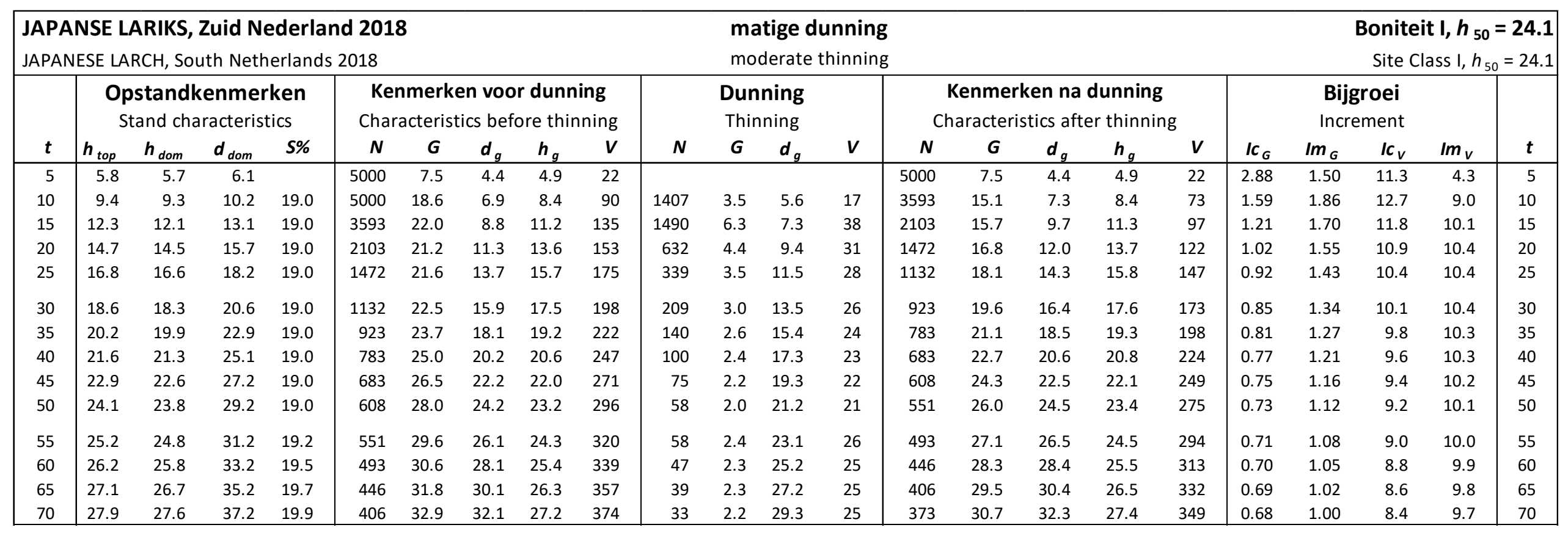




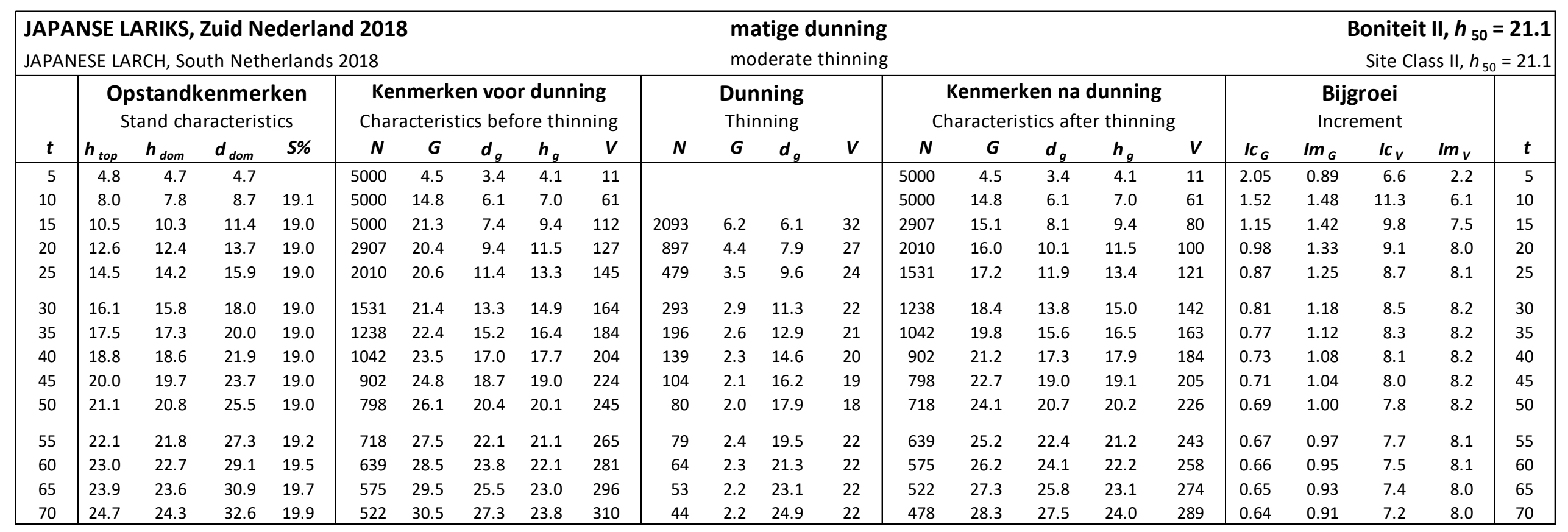




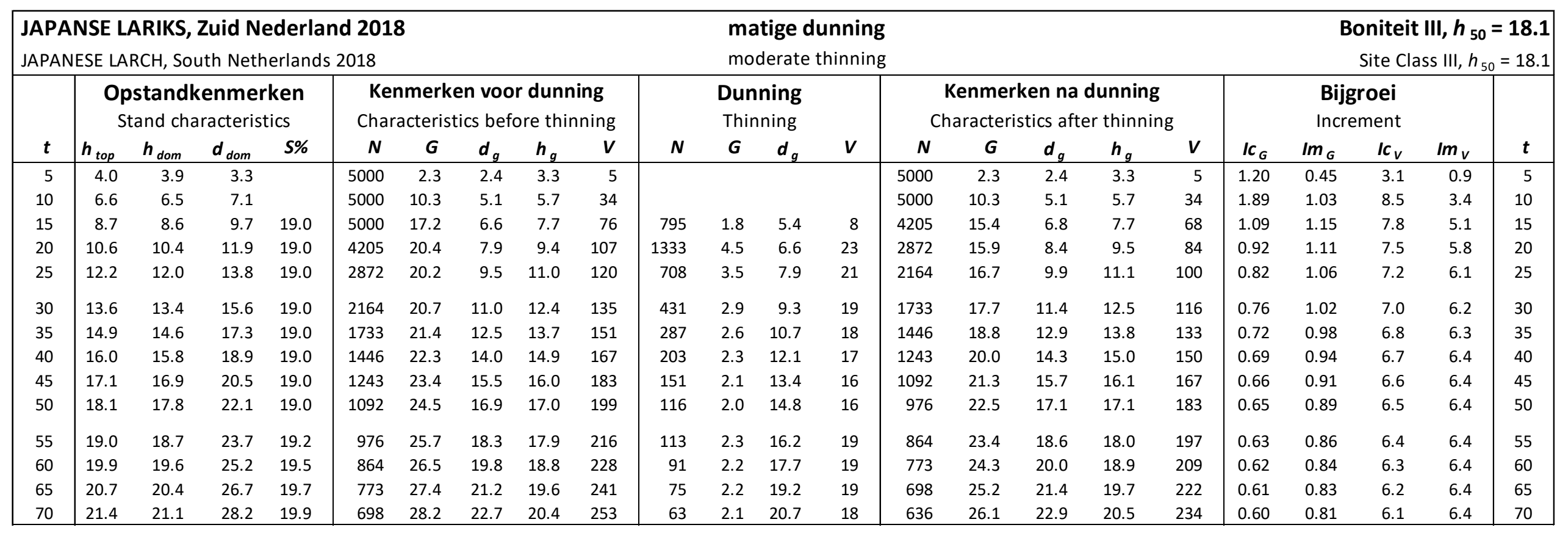




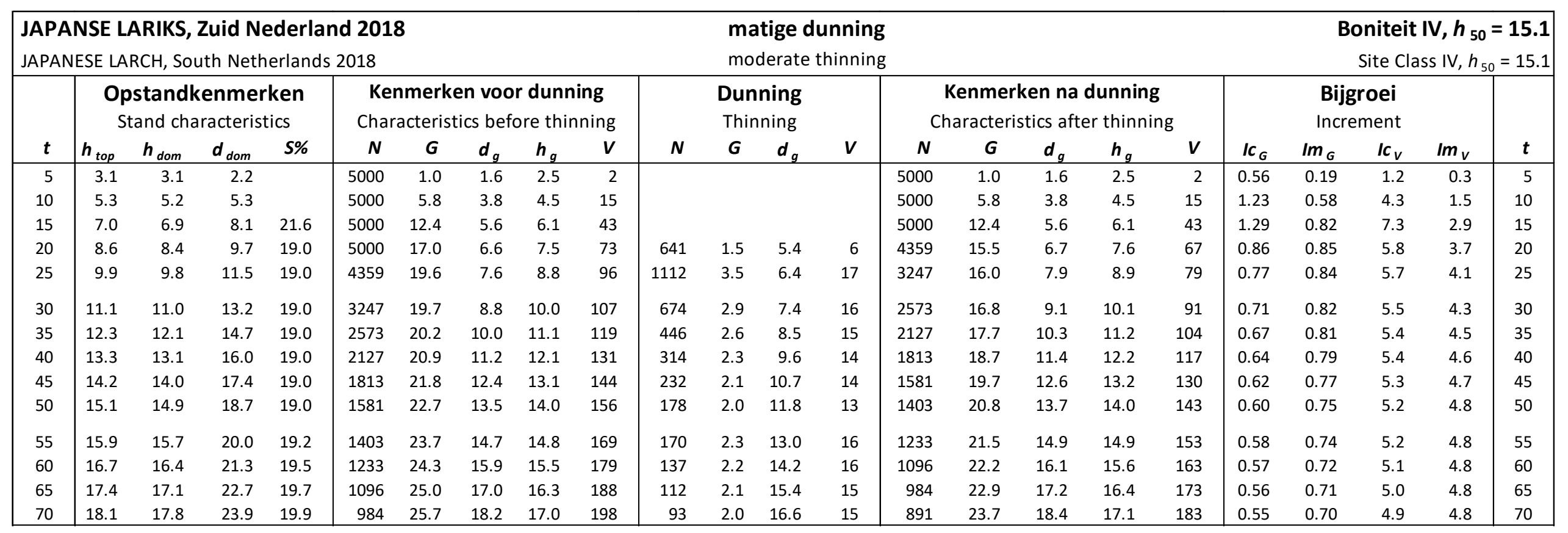




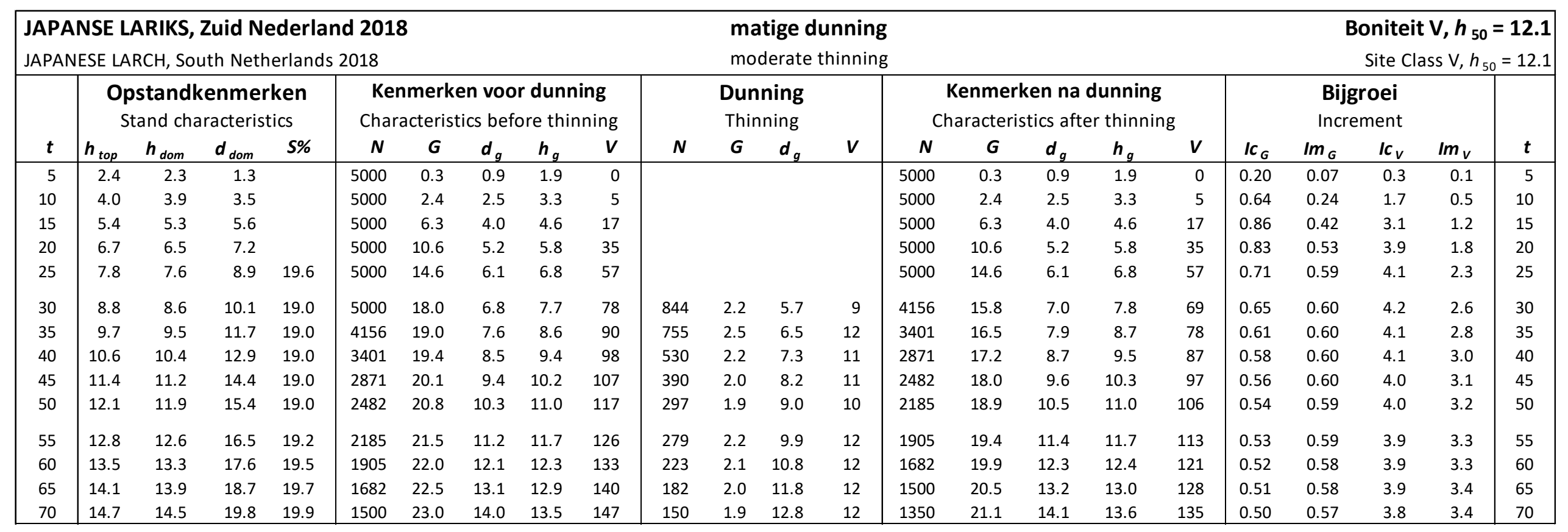




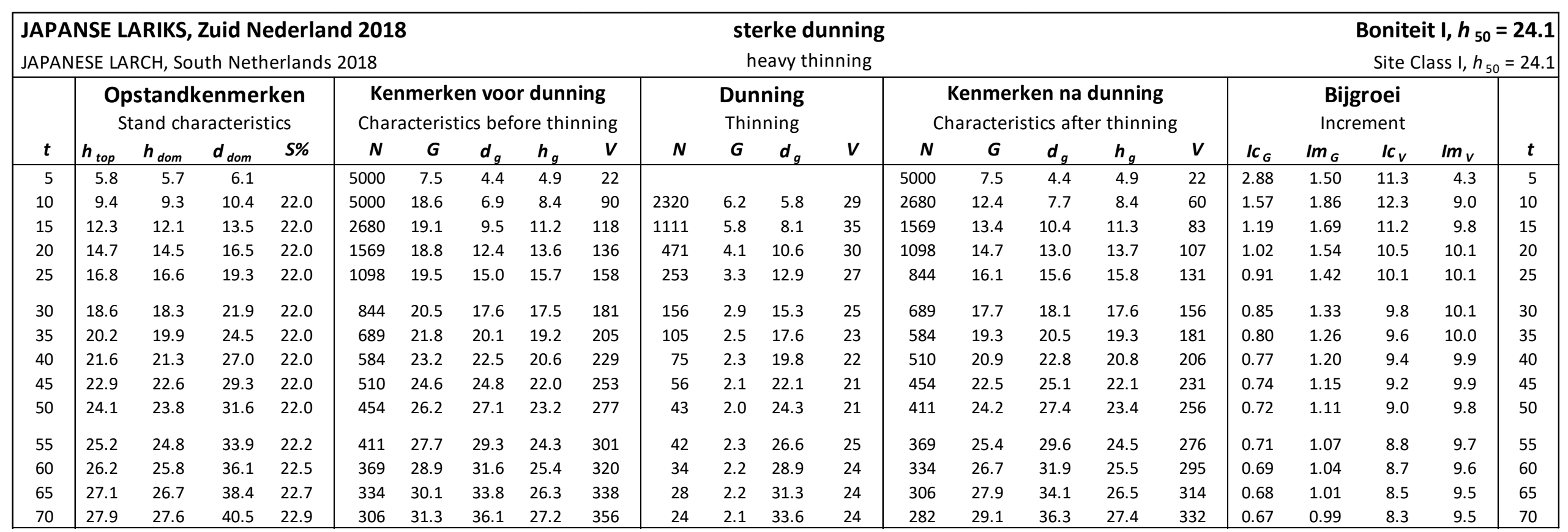




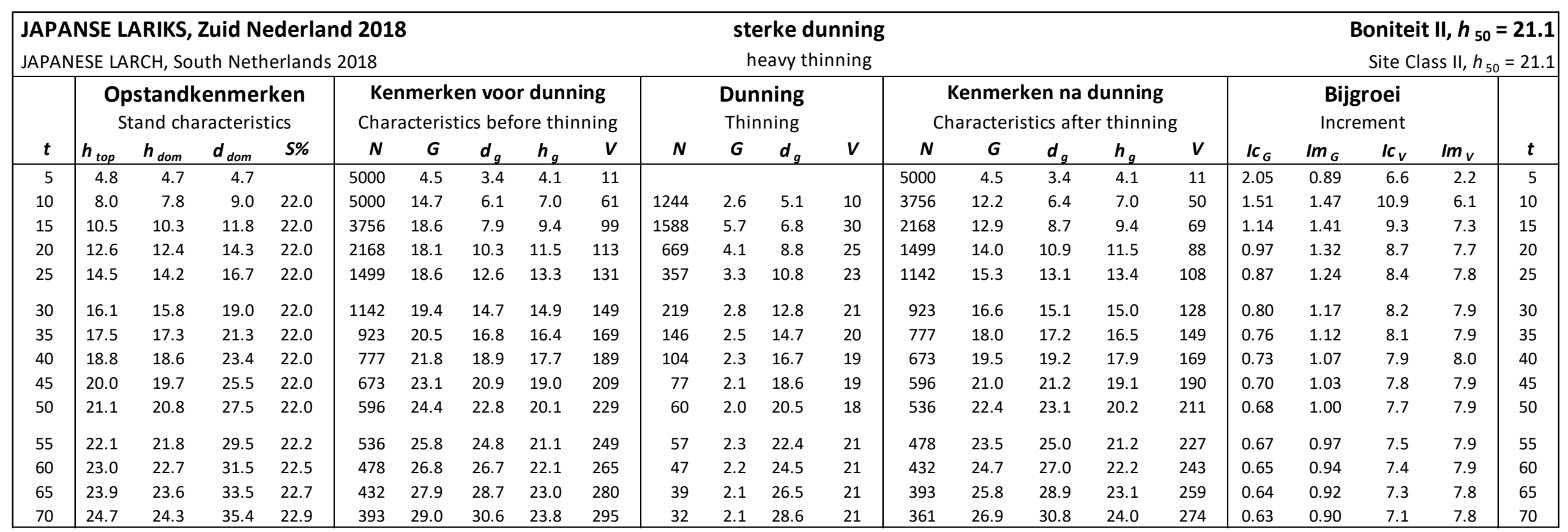




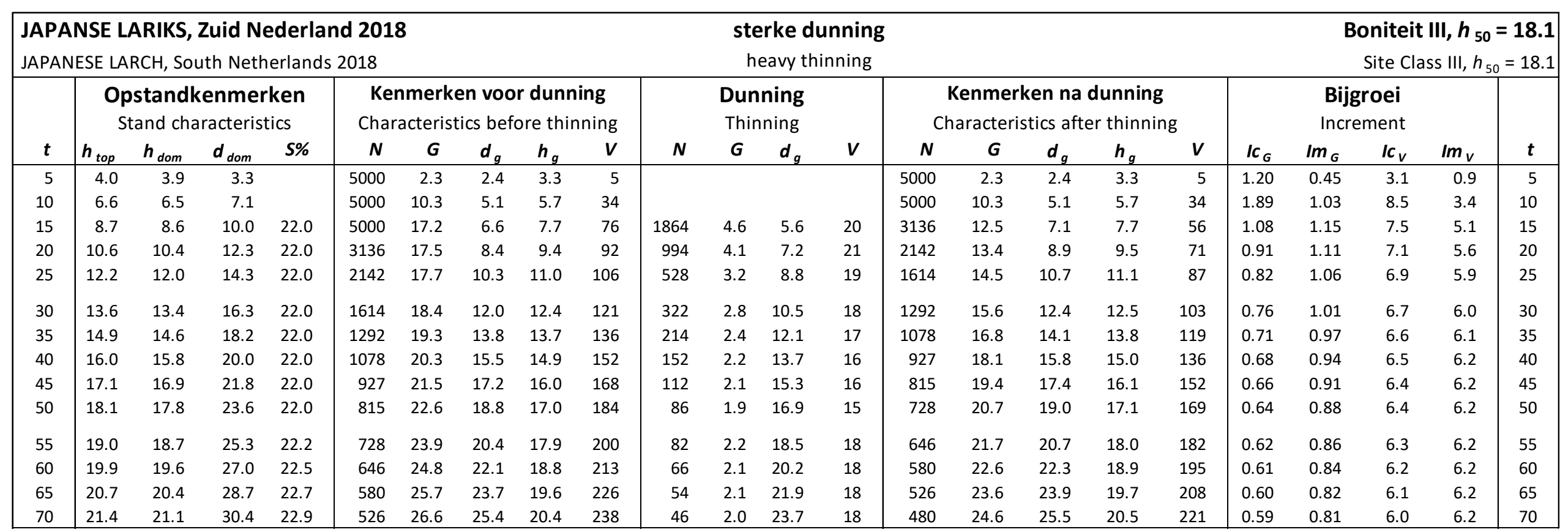




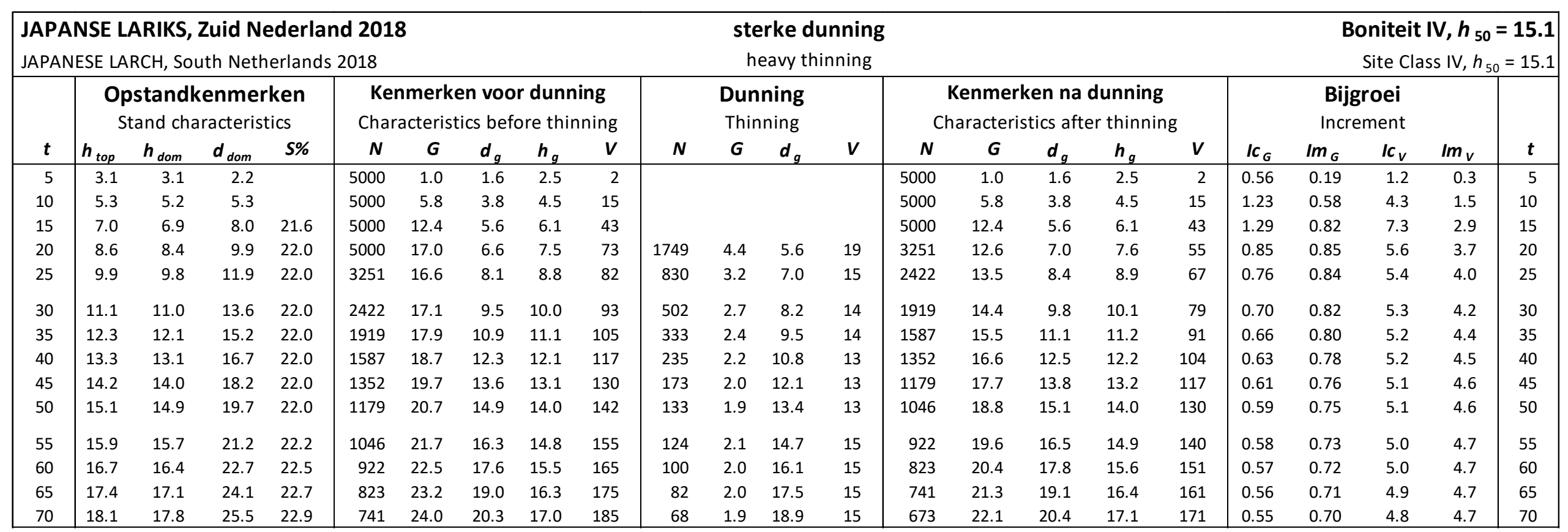




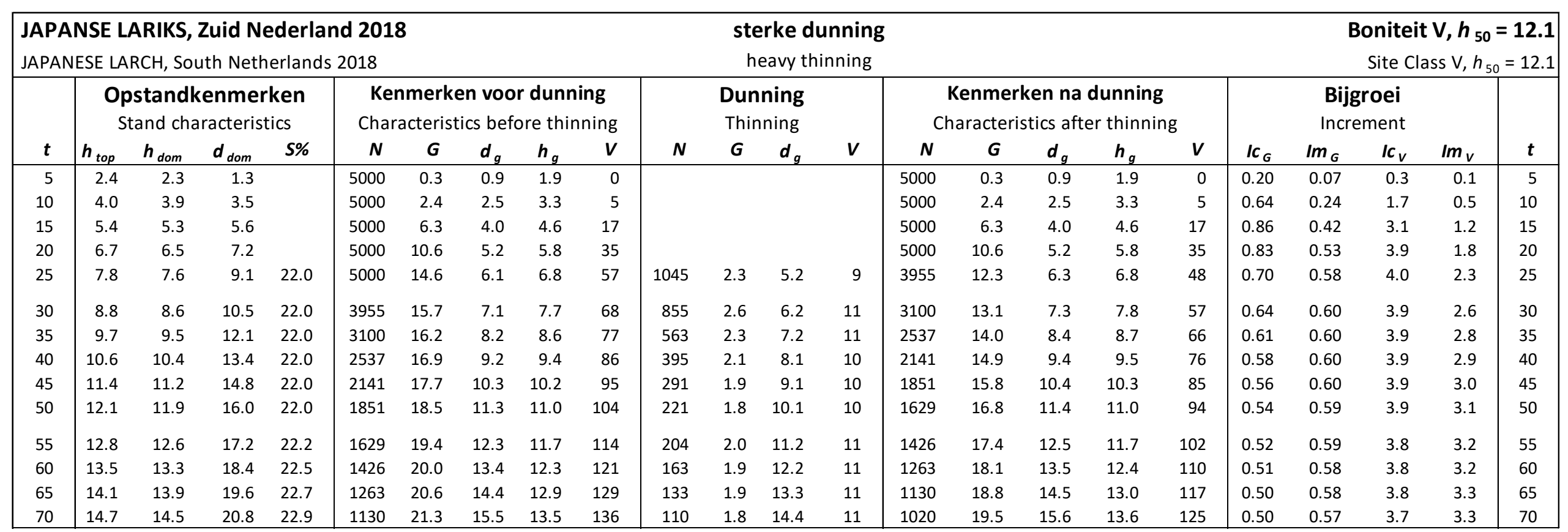




\section{Fijnspar (Picea abies)}

\section{Norway spruce}

Jansen, J.J., G.M.J. Mohren, A. Oosterbaan en J. den Ouden

Bron: Jansen, J.J., G.M.J. Mohren, A. Oosterbaan en J. den Ouden, 2018. Groei en productie van fijnspar in Nederland. FEM Groei en Productie Rapport 2018 - 2, 88 blz.

Dit rapport is gratis te downloaden op: https://doi.org/10.18174/444089

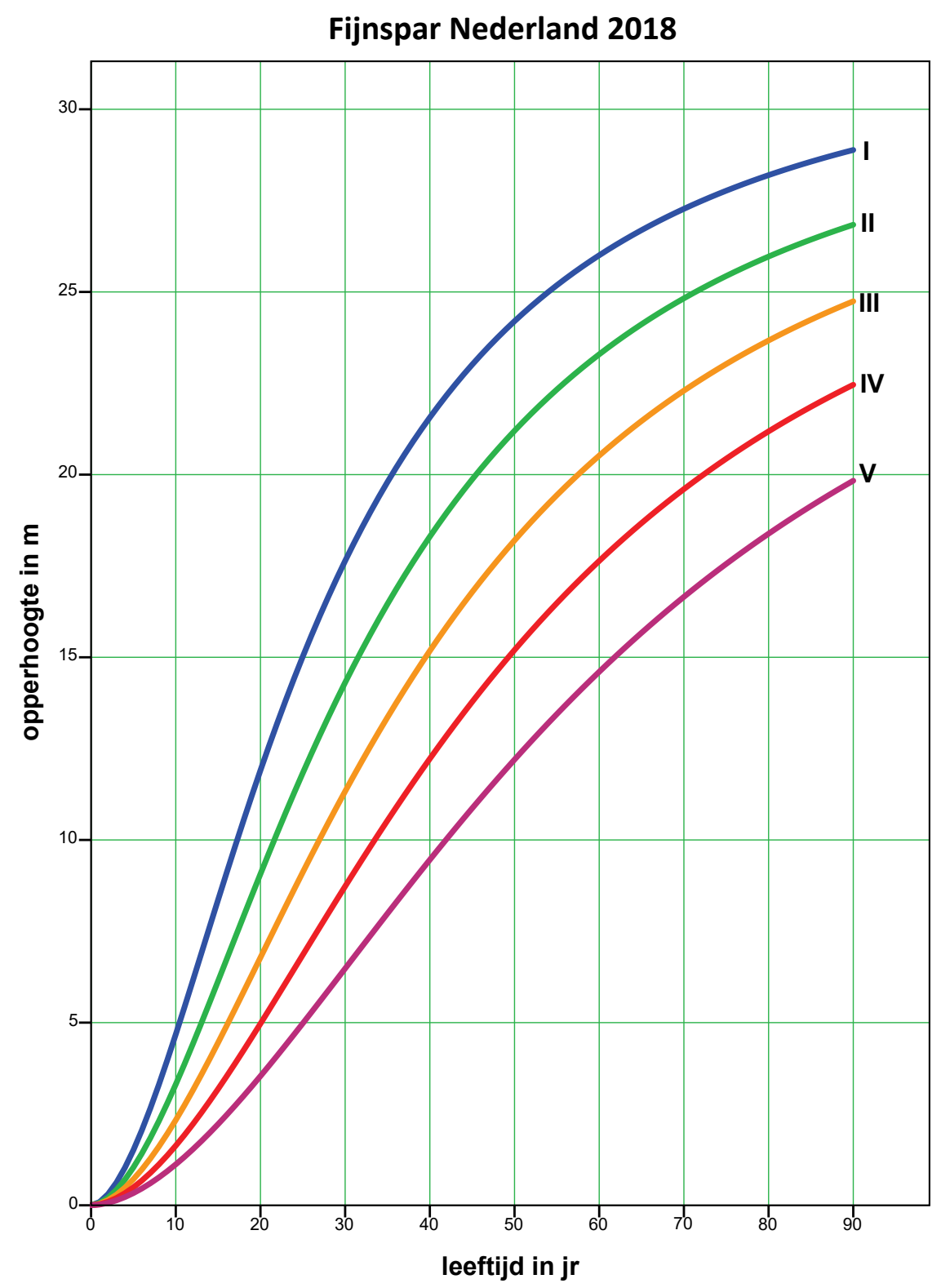




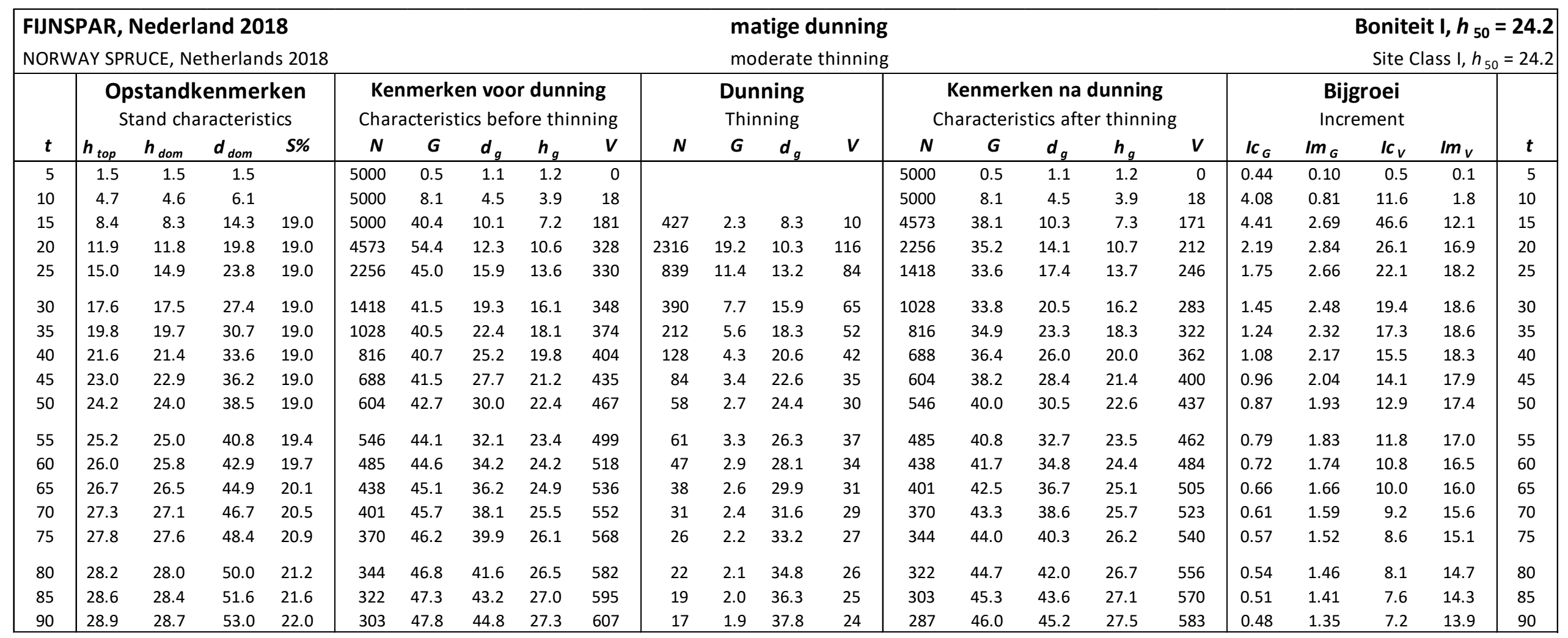




\begin{tabular}{|c|c|c|c|c|c|c|c|c|c|c|c|c|c|c|c|c|c|c|c|c|c|c|c|}
\hline \multirow{2}{*}{\multicolumn{5}{|c|}{$\begin{array}{l}\text { FIJNSPAR, Nederland } 2018 \\
\text { NORWAY SPRUCE, Netherlands } 2018\end{array}$}} & \multicolumn{15}{|c|}{ matige dunning } & \multirow{2}{*}{\multicolumn{4}{|c|}{$\begin{array}{r}\text { Boniteit II, } \boldsymbol{h}_{\mathbf{5 0}}=\mathbf{2 1 . 2} \\
\text { Site Class II, } h_{50}=21.2\end{array}$}} \\
\hline & & & & & & & & & & & & derat & inni & & & & & & & & & & \\
\hline \multirow[b]{2}{*}{$t$} & \multicolumn{4}{|c|}{$\begin{array}{l}\text { Opstandkenmerken } \\
\text { Stand characteristics }\end{array}$} & \multicolumn{5}{|c|}{$\begin{array}{l}\text { Kenmerken voor dunning } \\
\text { Characteristics before thinning }\end{array}$} & \multicolumn{4}{|c|}{$\begin{array}{l}\text { Dunning } \\
\text { Thinning }\end{array}$} & \multicolumn{5}{|c|}{$\begin{array}{l}\text { Kenmerken na dunning } \\
\text { Characteristics after thinning }\end{array}$} & \multicolumn{4}{|c|}{$\begin{array}{c}\text { Bijgroei } \\
\text { Increment }\end{array}$} & \multirow[b]{2}{*}{$t$} \\
\hline & $\boldsymbol{h}_{\text {top }}$ & $\boldsymbol{h}_{\text {dom }}$ & $\boldsymbol{d}_{d o m}$ & S\% & $N$ & $\boldsymbol{G}$ & $d_{g}$ & $h_{g}$ & $V$ & $N$ & $\boldsymbol{G}$ & $d_{g}$ & $v$ & $N$ & $G$ & $d_{g}$ & $h_{g}$ & $v$ & $I c_{G}$ & $I m_{G}$ & $I c_{v}$ & $I m_{V}$ & \\
\hline 5 & 1.0 & 1.0 & & & 5000 & & & & 0 & & & & & 5000 & & & & 0 & & & 0.2 & 0.0 & 5 \\
\hline 10 & 3.3 & 3.3 & 3.6 & & 5000 & 2.8 & 2.7 & 2.6 & 5 & & & & & 5000 & 2.8 & 2.7 & 2.6 & 5 & 1.30 & 0.28 & 2.9 & 0.5 & 10 \\
\hline 15 & 6.1 & 6.1 & 9.7 & & 5000 & 20.1 & 7.2 & 5.2 & 56 & & & & & 5000 & 20.1 & 7.2 & 5.2 & 56 & 6.61 & 1.34 & 22.8 & 3.7 & 15 \\
\hline 20 & 9.1 & 9.0 & 15.7 & 19.0 & 5000 & 45.3 & 10.7 & 7.9 & 217 & 1112 & 6.8 & 8.8 & 33 & 3888 & 38.6 & 11.2 & 8.0 & 185 & 3.35 & 2.27 & 26.8 & 10.9 & 20 \\
\hline 25 & \begin{tabular}{|l|l}
11.8 \\
\end{tabular} & 11.8 & 19.8 & 19.0 & 3888 & 51.4 & 13.0 & 10.6 & 308 & 1604 & 14.6 & 10.7 & 88 & 2284 & 36.8 & 14.3 & 10.6 & 220 & 1.84 & 2.32 & 21.0 & 13.6 & 25 \\
\hline 30 & $\mid 14.3$ & 14.2 & 23.1 & 19.0 & 2284 & 45.2 & 15.9 & 12.9 & 318 & 722 & 9.7 & 13.1 & 68 & 1562 & 35.5 & 17.0 & 13.0 & 249 & 1.53 & 2.22 & 18.5 & 14.6 & 30 \\
\hline 35 & 16.5 & 16.3 & 26.1 & 19.0 & 1562 & 42.6 & 18.6 & 15.0 & 337 & 382 & 7.0 & 15.3 & 56 & 1181 & 35.6 & 19.6 & 15.1 & 281 & 1.31 & 2.10 & 16.7 & 15.0 & 35 \\
\hline 40 & $\mid 18.3$ & 18.2 & 28.9 & 19.0 & 1181 & 41.7 & 21.2 & 16.8 & 361 & 226 & 5.3 & 17.4 & 46 & 955 & 36.4 & 22.0 & 16.9 & 314 & 1.15 & 1.99 & 15.2 & 15.1 & 40 \\
\hline 45 & $\mid 19.9$ & 19.7 & 31.4 & 19.0 & 955 & 41.8 & 23.6 & 18.3 & 387 & 145 & 4.2 & 19.3 & 39 & 810 & 37.6 & 24.3 & 18.5 & 348 & 1.02 & 1.89 & 14.0 & 15.1 & 45 \\
\hline 50 & 21.2 & 21.1 & 33.7 & 19.0 & 810 & 42.4 & 25.8 & 19.6 & 415 & 98 & 3.4 & 21.0 & 34 & 712 & 39.0 & 26.4 & 19.8 & 381 & 0.92 & 1.80 & 12.9 & 14.9 & 50 \\
\hline 55 & 22.3 & 22.2 & 36.0 & 19.4 & 712 & 43.3 & 27.8 & 20.8 & 443 & 95 & 3.9 & 22.8 & 40 & 617 & 39.5 & 28.5 & 20.9 & 403 & 0.83 & 1.72 & 11.8 & 14.7 & 55 \\
\hline 60 & 23.3 & 23.1 & 38.1 & 19.7 & 617 & 43.4 & 29.9 & 21.7 & 460 & 71 & 3.4 & 24.6 & 36 & 546 & 40.0 & 30.6 & 21.9 & 424 & 0.76 & 1.64 & 10.9 & 14.4 & 60 \\
\hline 65 & 24.1 & 24.0 & 40.1 & 20.1 & 546 & 43.7 & 31.9 & 22.6 & 476 & 55 & 3.0 & 26.4 & 33 & 491 & 40.6 & 32.5 & 22.7 & 443 & 0.69 & 1.57 & 10.0 & 14.1 & 65 \\
\hline 70 & 24.8 & 24.7 & 42.0 & 20.5 & 491 & 44.0 & 33.8 & 23.3 & 492 & 44 & 2.7 & 28.0 & 31 & 446 & 41.3 & 34.3 & 23.5 & 461 & 0.64 & 1.50 & 9.3 & 13.8 & 70 \\
\hline 75 & 25.4 & 25.3 & 43.7 & 20.9 & 446 & 44.4 & 35.6 & 23.9 & 506 & 36 & 2.5 & 29.6 & 29 & 410 & 41.9 & 36.0 & 24.1 & 477 & 0.60 & 1.44 & 8.7 & 13.4 & 75 \\
\hline 80 & 26.0 & 25.8 & 45.4 & 21.2 & 410 & 44.7 & 37.3 & 24.5 & 519 & 30 & 2.3 & 31.2 & 27 & 380 & 42.4 & 37.7 & 24.7 & 493 & 0.56 & 1.39 & 8.1 & 13.1 & 80 \\
\hline 85 & 26.4 & 26.3 & 47.0 & 21.6 & 380 & 45.2 & 38.9 & 25.0 & 532 & 26 & 2.2 & 32.7 & 25 & 354 & 43.0 & 39.3 & 25.2 & 507 & 0.53 & 1.34 & 7.7 & 12.8 & 85 \\
\hline 90 & 26.8 & 26.7 & 48.5 & 22.0 & 354 & 45.6 & 40.5 & 25.5 & 544 & 22 & 2.0 & 34.1 & 24 & 332 & 43.5 & 40.9 & 25.6 & 520 & 0.50 & 1.29 & 7.2 & 12.5 & 90 \\
\hline
\end{tabular}




\begin{tabular}{|c|c|c|c|c|c|c|c|c|c|c|c|c|c|c|c|c|c|c|c|c|c|c|c|}
\hline \multicolumn{11}{|c|}{$\begin{array}{l}\text { FIJNSPAR, Nederland } 2018 \\
\text { NORWAY SPRUCE, Netherlands } 2018\end{array}$} & \multicolumn{9}{|c|}{$\begin{array}{l}\text { matige dunning } \\
\text { moderate thinning }\end{array}$} & \multicolumn{4}{|c|}{$\begin{array}{r}\text { Boniteit III, } \boldsymbol{h}_{\mathbf{5 0}}=\mathbf{1 8 . 2} \\
\text { Site Class III, } h_{50}=18.2\end{array}$} \\
\hline \multirow[b]{2}{*}{$t$} & \multicolumn{4}{|c|}{$\begin{array}{l}\text { Opstandkenmerken } \\
\text { Stand characteristics }\end{array}$} & \multicolumn{5}{|c|}{$\begin{array}{l}\text { Kenmerken voor dunning } \\
\text { Characteristics before thinning }\end{array}$} & \multicolumn{4}{|c|}{$\begin{array}{l}\text { Dunning } \\
\text { Thinning }\end{array}$} & \multicolumn{5}{|c|}{$\begin{array}{c}\text { Kenmerken na dunning } \\
\text { Characteristics after thinning }\end{array}$} & \multicolumn{4}{|c|}{$\begin{array}{l}\text { Bijgroei } \\
\text { Increment }\end{array}$} & \multirow[b]{2}{*}{$t$} \\
\hline & $h_{\text {top }}$ & $\boldsymbol{h}_{\text {dom }}$ & $\boldsymbol{d}_{d o m}$ & S\% & $N$ & $\boldsymbol{G}$ & $d_{g}$ & $h_{g}$ & $v$ & $N$ & $\boldsymbol{G}$ & $d_{g}$ & $v$ & $N$ & $\boldsymbol{G}$ & $d_{g}$ & $h_{g}$ & $v$ & $I c_{G}$ & $I m_{G}$ & $I c_{v}$ & $I m_{V}$ & \\
\hline 5 & 0.7 & 0.7 & & & 5000 & & & & 0 & & & & & 5000 & & & & 0 & & & 0.0 & 0.0 & 5 \\
\hline 10 & 2.3 & 2.3 & 2.3 & & 5000 & 1.2 & 1.7 & 1.9 & 1 & & & & & 5000 & 1.2 & 1.7 & 1.9 & 1 & 0.45 & 0.12 & 0.8 & 0.1 & 10 \\
\hline 15 & 4.5 & 4.4 & 5.7 & & 5000 & 6.9 & 4.2 & 3.7 & 15 & & & & & 5000 & 6.9 & 4.2 & 3.7 & 15 & 2.26 & 0.46 & 6.2 & 1.0 & 15 \\
\hline 20 & 6.8 & 6.7 & 11.4 & & 5000 & 28.3 & 8.5 & 5.8 & 85 & & & & & 5000 & 28.3 & 8.5 & 5.8 & 85 & 5.73 & 1.41 & 22.3 & 4.3 & 20 \\
\hline 25 & 9.1 & 9.1 & 15.8 & 19.0 & 5000 & 46.6 & 10.9 & 8.0 & 224 & 1152 & 7.2 & 8.9 & 35 & 3848 & 39.4 & 11.4 & 8.1 & 190 & 2.83 & 1.86 & 22.4 & 9.0 & 25 \\
\hline 30 & 11.3 & 11.3 & 19.2 & 19.0 & 3848 & 50.8 & 13.0 & 10.1 & 294 & 1357 & 12.2 & 10.7 & 71 & 2492 & 38.6 & 14.0 & 10.2 & 223 & 1.69 & 1.93 & 17.9 & 10.9 & 30 \\
\hline 35 & \begin{tabular}{|l|l}
13.4 \\
\end{tabular} & 13.3 & 21.9 & 19.0 & 2492 & 46.0 & 15.3 & 12.1 & 306 & 699 & 8.7 & 12.6 & 58 & 1793 & 37.3 & 16.3 & 12.2 & 248 & 1.39 & 1.87 & 15.9 & 11.7 & 35 \\
\hline 40 & 15.2 & 15.1 & 24.4 & 19.0 & 1793 & 43.8 & 17.6 & 13.9 & 324 & 404 & 6.6 & 14.5 & 49 & 1389 & 37.2 & 18.5 & 14.0 & 274 & 1.21 & 1.80 & 14.7 & 12.2 & 40 \\
\hline 45 & \begin{tabular}{|l|l} 
& 16.8
\end{tabular} & 16.7 & 26.8 & 19.0 & 1389 & 42.9 & 19.8 & 15.4 & 345 & 254 & 5.2 & 16.2 & 42 & 1135 & 37.6 & 20.5 & 15.5 & 303 & 1.08 & 1.73 & 13.6 & 12.4 & 45 \\
\hline 50 & 18.2 & 18.1 & 29.0 & 19.0 & 1135 & 42.8 & 21.9 & 16.8 & 368 & 170 & 4.3 & 17.9 & 37 & 966 & 38.5 & 22.5 & 16.9 & 331 & 0.97 & 1.66 & 12.7 & 12.5 & 50 \\
\hline 55 & 19.4 & 19.3 & 31.3 & 19.4 & 966 & 43.1 & 23.8 & 18.1 & 392 & 151 & 4.5 & 19.6 & 42 & 814 & 38.6 & 24.6 & 18.2 & 351 & 0.88 & 1.59 & 11.7 & 12.4 & 55 \\
\hline 60 & 20.5 & 20.4 & 33.4 & 19.7 & 814 & 42.8 & 25.9 & 19.1 & 407 & 111 & 4.0 & 21.3 & 38 & 704 & 38.8 & 26.5 & 19.3 & 370 & 0.80 & 1.53 & 10.9 & 12.3 & 60 \\
\hline 65 & 21.5 & 21.3 & 35.4 & 20.1 & 704 & 42.6 & 27.8 & 20.1 & 422 & 84 & 3.5 & 23.0 & 35 & 619 & 39.2 & 28.4 & 20.2 & 387 & 0.73 & 1.47 & 10.1 & 12.2 & 65 \\
\hline 70 & 22.3 & 22.1 & 37.3 & 20.5 & 619 & 42.7 & 29.6 & 20.9 & 436 & 66 & 3.1 & 24.6 & 32 & 553 & 39.5 & 30.2 & 21.1 & 404 & 0.68 & 1.41 & 9.4 & 12.0 & 70 \\
\hline 75 & 23.0 & 22.9 & 39.1 & 20.9 & 553 & 42.8 & 31.4 & 21.7 & 449 & 53 & 2.8 & 26.2 & 30 & 501 & 40.0 & 31.9 & 21.8 & 419 & 0.63 & 1.36 & 8.8 & 11.8 & 75 \\
\hline 80 & 23.7 & 23.5 & 40.8 & 21.2 & 501 & 43.0 & 33.1 & 22.4 & 462 & 43 & 2.6 & 27.7 & 28 & 457 & 40.4 & 33.5 & 22.5 & 434 & 0.59 & 1.32 & 8.2 & 11.6 & 80 \\
\hline 85 & 24.2 & 24.1 & 42.4 & 21.6 & 457 & 43.2 & 34.7 & 23.0 & 474 & 36 & 2.4 & 29.1 & 26 & 421 & 40.8 & 35.1 & 23.1 & 447 & 0.55 & 1.27 & 7.8 & 11.4 & 85 \\
\hline 90 & 24.7 & 24.6 & 43.9 & 22.0 & 421 & 43.5 & 36.3 & 23.5 & 485 & 31 & 2.2 & 30.6 & 25 & 391 & 41.3 & 36.7 & 23.7 & 460 & 0.52 & 1.23 & 7.3 & 11.2 & 90 \\
\hline
\end{tabular}




\begin{tabular}{|c|c|c|c|c|c|c|c|c|c|c|c|c|c|c|c|c|c|c|c|c|c|c|c|}
\hline \multicolumn{11}{|c|}{$\begin{array}{l}\text { FIJNSPAR, Nederland } 2018 \\
\text { NORWAY SPRUCE Netherlands } 2018\end{array}$} & \multicolumn{9}{|c|}{ matige dunning } & \multicolumn{4}{|c|}{ Boniteit IV, $h_{50}=15.2$} \\
\hline \multirow[b]{2}{*}{$t$} & \multicolumn{4}{|c|}{$\begin{array}{c}\text { Opstandkenmerken } \\
\text { Stand characteristics }\end{array}$} & \multicolumn{5}{|c|}{$\begin{array}{l}\text { Kenmerken voor dunning } \\
\text { Characteristics before thinning }\end{array}$} & \multicolumn{4}{|c|}{$\begin{array}{l}\text { Dunning } \\
\text { Thinning }\end{array}$} & \multicolumn{5}{|c|}{$\begin{array}{c}\text { Kenmerken na dunning } \\
\text { Characteristics after thinning }\end{array}$} & \multicolumn{4}{|c|}{$\begin{array}{l}\text { Bijgroei } \\
\text { Increment }\end{array}$} & \multirow[b]{2}{*}{$t$} \\
\hline & $h_{\text {top }}$ & $\boldsymbol{h}_{\text {dom }}$ & $d_{d o m}$ & $S \%$ & $N$ & $\boldsymbol{G}$ & $d_{g}$ & $h_{g}$ & $v$ & $N$ & $\boldsymbol{G}$ & $d_{g}$ & $v$ & $N$ & $\boldsymbol{G}$ & $d_{g}$ & $\boldsymbol{h}_{g}$ & $v$ & $I c_{G}$ & $I m_{G}$ & $I c_{v}$ & $I m_{V}$ & \\
\hline 5 & 0.5 & 0.5 & & & 5000 & & & & 0 & & & & & 5000 & & & & 0 & & & 0.0 & 0.0 & 5 \\
\hline 10 & 1.6 & 1.6 & 1.6 & & 5000 & 0.6 & 1.2 & 1.3 & 1 & & & & & 5000 & 0.6 & 1.2 & 1.3 & 1 & 0.17 & 0.06 & 0.2 & 0.1 & 10 \\
\hline 15 & 3.2 & 3.2 & 3.4 & & 5000 & 2.5 & 2.5 & 2.5 & 4 & & & & & 5000 & 2.5 & 2.5 & 2.5 & 4 & 0.75 & 0.17 & 1.6 & 0.3 & 15 \\
\hline 20 & 5.0 & 4.9 & 6.8 & & 5000 & 9.9 & 5.0 & 4.2 & 28 & & & & & 5000 & 9.9 & 5.0 & 4.2 & 28 & 2.44 & 0.49 & 7.2 & 1.4 & 20 \\
\hline 25 & 6.9 & 6.8 & 11.7 & & 5000 & 29.3 & 8.6 & 5.9 & 90 & & & & & 5000 & 29.3 & 8.6 & 5.9 & 90 & 4.69 & 1.17 & 18.5 & 3.6 & 25 \\
\hline 30 & 8.7 & 8.7 & 15.0 & 19.0 & 5000 & 45.1 & 10.7 & 7.7 & 209 & 804 & 4.8 & 8.7 & 23 & 4196 & 40.2 & 11.0 & 7.7 & 187 & 2.57 & 1.50 & 19.0 & 7.0 & 30 \\
\hline 35 & 10.5 & 10.5 & 18.0 & 19.0 & 4196 & 51.2 & 12.5 & 9.4 & 279 & 1314 & 10.9 & 10.3 & 59 & 2883 & 40.4 & 13.4 & 9.5 & 219 & 1.83 & 1.60 & 16.9 & 8.6 & 35 \\
\hline 40 & 12.2 & 12.1 & 20.3 & 19.0 & 2883 & 47.6 & 14.5 & 11.1 & 294 & 742 & 8.3 & 11.9 & 51 & 2141 & 39.4 & 15.3 & 11.1 & 242 & 1.28 & 1.58 & 13.9 & 9.4 & 40 \\
\hline 45 & \begin{tabular}{|l|}
13.8 \\
\end{tabular} & 13.7 & 22.5 & 19.0 & 2141 & 45.4 & 16.4 & 12.6 & 309 & 456 & 6.5 & 13.5 & 44 & 1684 & 38.9 & 17.1 & 12.7 & 265 & 1.14 & 1.54 & 13.0 & 9.8 & 45 \\
\hline 50 & 15.2 & 15.1 & 24.6 & 19.0 & 1684 & 44.3 & 18.3 & 14.0 & 328 & 300 & 5.3 & 15.0 & 39 & 1384 & 39.0 & 18.9 & 14.1 & 289 & 1.03 & 1.50 & 12.2 & 10.1 & 50 \\
\hline 55 & 16.5 & 16.4 & 26.7 & 19.4 & 1384 & 43.9 & 20.1 & 15.3 & 348 & 252 & 5.4 & 16.5 & 43 & 1133 & 38.5 & 20.8 & 15.4 & 305 & 0.93 & 1.45 & 11.4 & 10.3 & 55 \\
\hline 60 & 17.6 & 17.5 & 28.7 & 19.7 & 1133 & 43.0 & 22.0 & 16.4 & 360 & 180 & 4.7 & 18.1 & 39 & 952 & 38.3 & 22.6 & 16.5 & 321 & 0.85 & 1.40 & 10.7 & 10.3 & 60 \\
\hline 65 & 18.7 & 18.5 & 30.7 & 20.1 & 952 & 42.4 & 23.8 & 17.5 & 373 & 134 & 4.1 & 19.7 & 36 & 818 & 38.3 & 24.4 & 17.6 & 337 & 0.77 & 1.36 & 10.0 & 10.3 & 65 \\
\hline 70 & 19.6 & 19.5 & 32.5 & 20.5 & 818 & 42.0 & 25.6 & 18.4 & 385 & 102 & 3.6 & 21.2 & 33 & 716 & 38.4 & 26.1 & 18.5 & 351 & 0.71 & 1.31 & 9.3 & 10.3 & 70 \\
\hline 75 & 20.4 & 20.3 & 34.3 & 20.9 & 716 & 41.8 & 27.3 & 19.3 & 397 & 80 & 3.3 & 22.7 & 31 & 636 & 38.5 & 27.8 & 19.4 & 366 & 0.66 & 1.27 & 8.8 & 10.2 & 75 \\
\hline 80 & 21.2 & 21.0 & 36.0 & 21.2 & 636 & 41.7 & 28.9 & 20.0 & 408 & 65 & 3.0 & 24.2 & 29 & 571 & 38.8 & 29.4 & 20.2 & 379 & 0.62 & 1.23 & 8.3 & 10.1 & 80 \\
\hline 85 & 21.9 & 21.7 & 37.6 & 21.6 & 571 & 41.7 & 30.5 & 20.7 & 419 & 53 & 2.7 & 25.6 & 27 & 518 & 39.0 & 31.0 & 20.9 & 392 & 0.58 & 1.19 & 7.8 & 10.0 & 85 \\
\hline 90 & 22.5 & 22.3 & 39.2 & 22.0 & 518 & 41.8 & 32.1 & 21.4 & 430 & 44 & 2.5 & 27.0 & 26 & 474 & 39.3 & 32.5 & 21.5 & 404 & 0.54 & 1.16 & 7.4 & 9.8 & 90 \\
\hline
\end{tabular}




\begin{tabular}{|c|c|c|c|c|c|c|c|c|c|c|c|c|c|c|c|c|c|c|c|c|c|c|c|}
\hline \multirow{2}{*}{\multicolumn{5}{|c|}{$\begin{array}{l}\text { FIJNSPAR, Nederland } 2018 \\
\text { NORWAY SPRUCE, Netherlands } 2018 \\
\end{array}$}} & \multicolumn{15}{|c|}{ matige dunning } & \multirow{2}{*}{\multicolumn{4}{|c|}{$\begin{array}{r}\text { Boniteit V, } \boldsymbol{h}_{\mathbf{5 0}}=\mathbf{1 2 . 2} \\
\text { Site Class V, } h_{50}=12.2\end{array}$}} \\
\hline & & & & & & & & & & & & derat & inni & & & & & & & & & & \\
\hline \multirow[b]{2}{*}{$t$} & \multicolumn{4}{|c|}{$\begin{array}{l}\text { Opstandkenmerken } \\
\text { Stand characteristics }\end{array}$} & \multicolumn{5}{|c|}{$\begin{array}{l}\text { Kenmerken voor dunning } \\
\text { Characteristics before thinning }\end{array}$} & \multicolumn{4}{|c|}{$\begin{array}{l}\text { Dunning } \\
\text { Thinning }\end{array}$} & \multicolumn{5}{|c|}{$\begin{array}{l}\text { Kenmerken na dunning } \\
\text { Characteristics after thinning }\end{array}$} & \multicolumn{4}{|c|}{$\begin{array}{c}\text { Bijgroei } \\
\text { Increment }\end{array}$} & \multirow[b]{2}{*}{$t$} \\
\hline & $\boldsymbol{h}_{\text {top }}$ & $\boldsymbol{h}_{\text {dom }}$ & $\boldsymbol{d}_{\text {dom }}$ & $S \%$ & $N$ & $\boldsymbol{G}$ & $d_{g}$ & $h_{g}$ & $v$ & $N$ & $\boldsymbol{G}$ & $d_{g}$ & $v$ & $N$ & $\boldsymbol{G}$ & $d_{g}$ & $h_{g}$ & $v$ & $I c_{G}$ & $I m_{G}$ & $I c_{v}$ & $I m_{V}$ & \\
\hline 5 & 0.3 & 0.3 & & & 5000 & & & & 0 & & & & & 5000 & & & & 0 & & & 0.0 & 0.0 & 5 \\
\hline 10 & 1.1 & 1.1 & & & 5000 & & & & 0 & & & & & 5000 & & & & 0 & & & 0.2 & 0.0 & 10 \\
\hline 15 & 2.2 & 2.2 & 2.2 & & 5000 & 1.0 & 1.6 & 1.8 & 1 & & & & & 5000 & 1.0 & 1.6 & 1.8 & 1 & 0.26 & 0.07 & 0.4 & 0.1 & 15 \\
\hline 20 & 3.5 & 3.5 & 4.0 & & 5000 & 3.4 & 3.0 & 2.9 & 7 & & & & & 5000 & 3.4 & 3.0 & 2.9 & 7 & 0.79 & 0.17 & 1.8 & 0.4 & 20 \\
\hline 25 & 5.0 & 4.9 & 6.8 & & 5000 & 9.9 & 5.0 & 4.2 & 24 & & & & & 5000 & 9.9 & 5.0 & 4.2 & 24 & 1.95 & 0.40 & 5.8 & 0.9 & 25 \\
\hline 30 & 6.5 & 6.4 & 10.6 & & 5000 & 24.2 & 7.8 & 5.6 & 71 & & & & & 5000 & 24.2 & 7.8 & 5.6 & 71 & 3.87 & 0.81 & 14.0 & 2.4 & 30 \\
\hline 35 & 8.0 & 7.9 & 13.7 & 19.0 & 5000 & 40.2 & 10.1 & 7.0 & 173 & & & & & 5000 & 40.2 & 10.1 & 7.0 & 173 & 2.47 & 1.15 & 16.1 & 5.0 & 35 \\
\hline 40 & 9.5 & 9.4 & 16.2 & 19.0 & 5000 & 51.3 & 11.4 & 8.4 & 255 & 1424 & 9.9 & 9.4 & 49 & 3576 & 41.4 & 12.1 & 8.5 & 206 & 1.94 & 1.28 & 15.6 & 6.4 & 40 \\
\hline 45 & 10.9 & 10.8 & 18.6 & 19.0 & 3576 & 49.8 & 13.3 & 9.8 & 278 & 867 & 8.1 & 10.9 & 46 & 2708 & 41.7 & 14.0 & 9.9 & 233 & 1.39 & 1.33 & 13.2 & 7.3 & 45 \\
\hline 50 & 12.2 & 12.1 & 20.2 & 19.0 & 2708 & 47.5 & 14.9 & 11.1 & 292 & 559 & 6.6 & 12.2 & 41 & 2149 & 40.9 & 15.6 & 11.2 & 252 & 1.08 & 1.31 & 11.5 & 7.7 & 50 \\
\hline 55 & 13.4 & 13.4 & 22.1 & 19.4 & 2149 & 46.1 & 16.5 & 12.3 & 308 & 447 & 6.5 & 13.6 & 43 & 1702 & 39.6 & 17.2 & 12.4 & 264 & 0.98 & 1.29 & 10.9 & 8.1 & 55 \\
\hline 60 & 14.6 & 14.5 & 24.0 & 19.7 & 1702 & 44.3 & 18.2 & 13.5 & 317 & 313 & 5.5 & 15.0 & 40 & 1389 & 38.7 & 18.8 & 13.6 & 277 & 0.89 & 1.26 & 10.2 & 8.3 & 60 \\
\hline 65 & 15.7 & 15.6 & 25.8 & 20.1 & 1389 & 43.0 & 19.9 & 14.6 & 327 & 227 & 4.8 & 16.5 & 37 & 1162 & 38.2 & 20.5 & 14.7 & 290 & 0.82 & 1.23 & 9.6 & 8.4 & 65 \\
\hline 70 & $\mid 16.7$ & 16.5 & 27.6 & 20.5 & 1162 & 42.1 & 21.5 & 15.6 & 337 & 170 & 4.3 & 17.9 & 34 & 992 & 37.9 & 22.0 & 15.7 & 302 & 0.76 & 1.19 & 9.1 & 8.5 & 70 \\
\hline 75 & 17.6 & 17.4 & 29.3 & 20.9 & 992 & 41.5 & 23.1 & 16.5 & 347 & 131 & 3.8 & 19.3 & 32 & 861 & 37.7 & 23.6 & 16.6 & 315 & 0.70 & 1.16 & 8.6 & 8.5 & 75 \\
\hline 80 & 18.4 & 18.3 & 30.9 & 21.2 & 861 & 41.1 & 24.6 & 17.4 & 356 & 103 & 3.4 & 20.7 & 30 & 758 & 37.6 & 25.1 & 17.5 & 326 & 0.65 & 1.13 & 8.2 & 8.5 & 80 \\
\hline 85 & $\mid$\begin{tabular}{|l|}
19.1 \\
\end{tabular} & 19.0 & 32.5 & 21.6 & 758 & 40.8 & 26.2 & 18.1 & 366 & 83 & 3.1 & 22.0 & 28 & 676 & 37.6 & 26.6 & 18.3 & 338 & 0.61 & 1.10 & 7.7 & 8.4 & 85 \\
\hline 90 & 19.8 & 19.7 & 34.1 & 22.0 & 676 & 40.6 & 27.7 & 18.9 & 376 & 67 & 2.9 & 23.4 & 27 & 608 & 37.7 & 28.1 & 19.0 & 349 & 0.57 & 1.07 & 7.4 & 8.4 & 90 \\
\hline
\end{tabular}




\begin{tabular}{|c|c|c|c|c|c|c|c|c|c|c|c|c|c|c|c|c|c|c|c|c|c|c|c|}
\hline \multirow{2}{*}{\multicolumn{5}{|c|}{$\begin{array}{l}\text { FIJNSPAR, Nederland } 2018 \\
\text { NORWAY SPRUCE, Netherlands } 2018\end{array}$}} & \multicolumn{15}{|c|}{ sterke dunning } & \multicolumn{4}{|c|}{ Boniteit I, $h_{50}=24.2$} \\
\hline & & & & & & & & & & & & eavy $\mathrm{t}$ & nning & & & & & & & & Site & ss I, $h$ & \\
\hline \multirow[b]{2}{*}{$t$} & \multicolumn{4}{|c|}{$\begin{array}{c}\text { Opstandkenmerken } \\
\text { Stand characteristics }\end{array}$} & \multicolumn{5}{|c|}{$\begin{array}{l}\text { Kenmerken voor dunning } \\
\text { Characteristics before thinning }\end{array}$} & \multicolumn{4}{|c|}{$\begin{array}{l}\text { Dunning } \\
\text { Thinning }\end{array}$} & \multicolumn{5}{|c|}{$\begin{array}{l}\text { Kenmerken na dunning } \\
\text { Characteristics after thinning }\end{array}$} & \multicolumn{4}{|c|}{$\begin{array}{l}\text { Bijgroei } \\
\text { Increment }\end{array}$} & \multirow[b]{2}{*}{$t$} \\
\hline & $h_{\text {top }}$ & $\boldsymbol{h}_{\text {dom }}$ & $d_{d o m}$ & $S \%$ & $N$ & $\boldsymbol{G}$ & $d_{g}$ & $h_{g}$ & $\boldsymbol{V}$ & $N$ & $\boldsymbol{G}$ & $d_{g}$ & $\boldsymbol{V}$ & $N$ & $\boldsymbol{G}$ & $d_{g}$ & $h_{g}$ & $\boldsymbol{v}$ & $I c_{G}$ & $I m_{G}$ & $I c_{v}$ & $I m_{V}$ & \\
\hline 5 & 1.5 & 1.5 & 1.5 & & 5000 & 0.5 & 1.1 & 1.2 & 0 & & & & & 5000 & 0.5 & 1.1 & 1.2 & 0 & 0.44 & 0.10 & 0.5 & 0.1 & 5 \\
\hline 10 & 4.7 & 4.6 & 6.1 & & 5000 & 8.1 & 4.5 & 3.9 & 18 & & & & & 5000 & 8.1 & 4.5 & 3.9 & 18 & 4.08 & 0.81 & 11.6 & 1.8 & 10 \\
\hline 15 & 8.4 & 8.3 & 14.7 & 22.0 & 5000 & 39.8 & 10.1 & 7.2 & 178 & 1589 & 9.2 & 8.6 & 41 & 3411 & 30.6 & 10.7 & 7.3 & 137 & 4.10 & 2.65 & 43.7 & 11.9 & 15 \\
\hline 20 & 11.9 & 11.8 & 20.2 & 22.0 & 3411 & 45.7 & 13.1 & 10.6 & 275 & 1728 & 17.2 & 11.3 & 104 & 1683 & 28.5 & 14.7 & 10.7 & 171 & 2.04 & 2.75 & 22.9 & 15.8 & 20 \\
\hline 25 & 15.0 & 14.9 & 24.3 & 22.0 & 1683 & 37.6 & 16.9 & 13.6 & 275 & 625 & 10.2 & 14.4 & 75 & 1057 & 27.3 & 18.1 & 13.7 & 200 & 1.63 & 2.56 & 19.6 & 16.8 & 25 \\
\hline 30 & 17.6 & 17.5 & 28.2 & 22.0 & 1057 & 34.7 & 20.5 & 16.1 & 291 & 291 & 6.9 & 17.4 & 58 & 767 & 27.8 & 21.5 & 16.2 & 233 & 1.35 & 2.38 & 17.3 & 17.0 & 30 \\
\hline 35 & 19.8 & 19.7 & 31.7 & 22.0 & 767 & 34.0 & 23.8 & 18.1 & 314 & 158 & 5.1 & 20.2 & 47 & 609 & 29.0 & 24.6 & 18.3 & 267 & 1.15 & 2.22 & 15.5 & 16.9 & 35 \\
\hline 40 & 21.6 & 21.4 & 34.8 & 22.0 & 609 & 34.4 & 26.8 & 19.8 & 341 & 96 & 3.9 & 22.7 & 39 & 513 & 30.5 & 27.5 & 20.0 & 302 & 1.00 & 2.07 & 14.0 & 16.7 & 40 \\
\hline 45 & 23.0 & 22.9 & 37.6 & 22.0 & 513 & 35.2 & 29.6 & 21.2 & 369 & 62 & 3.1 & 25.0 & 32 & 451 & 32.1 & 30.1 & 21.4 & 337 & 0.89 & 1.95 & 12.7 & 16.3 & 45 \\
\hline 50 & 24.2 & 24.0 & 40.1 & 22.0 & 451 & 36.4 & 32.1 & 22.4 & 398 & 43 & 2.5 & 27.1 & 27 & 407 & 33.9 & 32.5 & 22.6 & 370 & 0.80 & 1.84 & 11.7 & 15.9 & 50 \\
\hline 55 & 25.2 & 25.0 & 42.5 & 22.4 & 407 & 37.7 & 34.3 & 23.4 & 427 & 44 & 2.9 & 29.1 & 33 & 364 & 34.8 & 34.9 & 23.5 & 394 & 0.73 & 1.74 & 10.7 & 15.5 & 55 \\
\hline 60 & 26.0 & 25.8 & 44.7 & 22.7 & 364 & 38.3 & 36.6 & 24.2 & 445 & 34 & 2.6 & 31.2 & 30 & 330 & 35.8 & 37.1 & 24.4 & 415 & 0.67 & 1.65 & 9.9 & 15.0 & 60 \\
\hline 65 & 26.7 & 26.5 & 46.8 & 23.1 & 330 & 39.0 & 38.8 & 24.9 & 463 & 27 & 2.3 & 33.1 & 27 & 303 & 36.7 & 39.2 & 25.1 & 435 & 0.62 & 1.58 & 9.1 & 14.6 & 65 \\
\hline 70 & 27.3 & 27.1 & 48.7 & 23.5 & 303 & 39.6 & 40.8 & 25.5 & 479 & 22 & 2.1 & 35.0 & 26 & 281 & 37.5 & 41.2 & 25.7 & 454 & 0.57 & 1.51 & 8.5 & 14.2 & 70 \\
\hline 75 & 27.8 & 27.6 & 50.5 & 23.9 & 281 & 40.3 & 42.7 & 26.1 & 495 & 18 & 1.9 & 36.7 & 24 & 263 & 38.4 & 43.1 & 26.2 & 471 & 0.54 & 1.44 & 7.9 & 13.8 & 75 \\
\hline 80 & 28.2 & 28.0 & 52.2 & 24.2 & 263 & 40.9 & 44.5 & 26.5 & 509 & 16 & 1.8 & 38.4 & 23 & 247 & 39.1 & 44.9 & 26.7 & 487 & 0.50 & 1.39 & 7.5 & 13.4 & 80 \\
\hline 85 & 28.6 & 28.4 & 53.9 & 24.6 & 247 & 41.6 & 46.3 & 27.0 & 523 & 14 & 1.7 & 40.1 & 22 & 234 & 39.9 & 46.6 & 27.1 & 501 & 0.48 & 1.33 & 7.0 & 13.0 & 85 \\
\hline 90 & 28.9 & 28.7 & 55.4 & 25.0 & 234 & 42.2 & 47.9 & 27.3 & 535 & 12 & 1.6 & 41.6 & 21 & 222 & 40.6 & 48.2 & 27.5 & 515 & 0.45 & 1.28 & 6.7 & 12.7 & 90 \\
\hline
\end{tabular}




\begin{tabular}{|c|c|c|c|c|c|c|c|c|c|c|c|c|c|c|c|c|c|c|c|c|c|c|c|}
\hline \multirow{2}{*}{\multicolumn{5}{|c|}{$\begin{array}{l}\text { FIJNSPAR, Nederland } 2018 \\
\text { NORWAY SPRUCE, Netherlands } 2018\end{array}$}} & \multicolumn{15}{|c|}{ sterke dunning } & \multirow{2}{*}{\multicolumn{4}{|c|}{$\begin{array}{r}\text { Boniteit II, } \boldsymbol{h}_{\mathbf{5 0}}=\mathbf{2 1 . 2} \\
\text { Site Class II, } h_{50}=21.2\end{array}$}} \\
\hline & & & & & & & & & & & & eavy t & ning & & & & & & & & & & \\
\hline \multirow[b]{2}{*}{$t$} & \multicolumn{4}{|c|}{$\begin{array}{c}\text { Opstandkenmerken } \\
\text { Stand characteristics }\end{array}$} & \multicolumn{5}{|c|}{$\begin{array}{l}\text { Kenmerken voor dunning } \\
\text { Characteristics before thinning }\end{array}$} & \multicolumn{4}{|c|}{$\begin{array}{c}\text { Dunning } \\
\text { Thinning }\end{array}$} & \multicolumn{5}{|c|}{$\begin{array}{l}\text { Kenmerken na dunning } \\
\text { Characteristics after thinning }\end{array}$} & \multicolumn{4}{|c|}{$\begin{array}{c}\text { Bijgroei } \\
\text { Increment }\end{array}$} & \multirow[b]{2}{*}{$t$} \\
\hline & $\boldsymbol{h}_{\text {top }}$ & $\boldsymbol{h}_{\text {dom }}$ & $\boldsymbol{d}_{d o m}$ & S\% & $N$ & $\boldsymbol{G}$ & $d_{g}$ & $h_{g}$ & $V$ & $N$ & $\boldsymbol{G}$ & $d_{g}$ & $v$ & $N$ & $\boldsymbol{G}$ & $d_{g}$ & $h_{g}$ & $v$ & $I c_{G}$ & $I m_{G}$ & $I c_{v}$ & $I m_{V}$ & \\
\hline 5 & 1.0 & 1.0 & & & 5000 & & & & 0 & & & & & 5000 & & & & 0 & & & 0.2 & 0.0 & 5 \\
\hline 10 & 3.3 & 3.3 & 3.6 & & 5000 & 2.8 & 2.7 & 2.6 & 5 & & & & & 5000 & 2.8 & 2.7 & 2.6 & 5 & 1.30 & 0.28 & 2.9 & 0.5 & 10 \\
\hline 15 & 6.1 & 6.1 & 9.7 & & 5000 & 20.1 & 7.2 & 5.2 & 56 & & & & & 5000 & 20.1 & 7.2 & 5.2 & 56 & 6.61 & 1.34 & 22.8 & 3.7 & 15 \\
\hline 20 & 9.1 & 9.0 & 16.0 & 22.0 & 5000 & 44.3 & 10.6 & 7.9 & 213 & 2100 & 13.7 & 9.1 & 66 & 2900 & 30.6 & 11.6 & 8.0 & 147 & 3.11 & 2.22 & 24.5 & 10.6 & 20 \\
\hline 25 & $\mid$\begin{tabular}{|l|}
11.8 \\
\end{tabular} & 11.8 & 20.2 & 22.0 & 2900 & 42.5 & 13.7 & 10.6 & 255 & 1196 & 12.9 & 11.7 & 78 & 1704 & 29.6 & 14.9 & 10.6 & 177 & 1.71 & 2.25 & 18.4 & 12.8 & 25 \\
\hline 30 & $\mid 14.3$ & 14.2 & 23.6 & 22.0 & 1704 & 37.4 & 16.7 & 12.9 & 263 & 538 & 8.6 & 14.3 & 61 & 1165 & 28.8 & 17.7 & 13.0 & 202 & 1.43 & 2.13 & 16.4 & 13.5 & 30 \\
\hline 35 & 16.5 & 16.3 & 26.8 & 22.0 & 1165 & 35.4 & 19.7 & 15.0 & 280 & 285 & 6.3 & 16.7 & 50 & 881 & 29.1 & 20.5 & 15.1 & 230 & 1.22 & 2.02 & 14.9 & 13.8 & 35 \\
\hline 40 & $\mid 18.3$ & 18.2 & 29.7 & 22.0 & 881 & 34.8 & 22.4 & 16.8 & 301 & 168 & 4.8 & 19.0 & 42 & 712 & 30.1 & 23.2 & 16.9 & 260 & 1.07 & 1.91 & 13.6 & 13.9 & 40 \\
\hline 45 & $\mid 19.9$ & 19.7 & 32.5 & 22.0 & 712 & 35.1 & 25.0 & 18.3 & 325 & 108 & 3.8 & 21.2 & 35 & 604 & 31.3 & 25.7 & 18.5 & 289 & 0.95 & 1.81 & 12.5 & 13.8 & 45 \\
\hline 50 & 21.2 & 21.1 & 35.0 & 22.0 & 604 & 35.7 & 27.4 & 19.6 & 350 & 73 & 3.1 & 23.2 & 30 & 531 & 32.6 & 28.0 & 19.8 & 319 & 0.85 & 1.72 & 11.6 & 13.6 & 50 \\
\hline 55 & 22.3 & 22.2 & 37.4 & 22.4 & 531 & 36.7 & 29.7 & 20.8 & 375 & 68 & 3.4 & 25.2 & 35 & 463 & 33.3 & 30.3 & 20.9 & 340 & 0.77 & 1.63 & 10.7 & 13.4 & 55 \\
\hline 60 & 23.3 & 23.1 & 39.6 & 22.7 & 463 & 37.0 & 31.9 & 21.7 & 392 & 51 & 3.0 & 27.2 & 32 & 411 & 34.0 & 32.4 & 21.9 & 360 & 0.70 & 1.56 & 9.9 & 13.1 & 60 \\
\hline 65 & 24.1 & 24.0 & 41.7 & 23.1 & 411 & 37.4 & 34.0 & 22.6 & 408 & 40 & 2.6 & 29.1 & 29 & 372 & 34.7 & 34.5 & 22.7 & 379 & 0.65 & 1.49 & 9.2 & 12.8 & 65 \\
\hline 70 & 24.8 & 24.7 & 43.7 & 23.5 & 372 & 37.9 & 36.0 & 23.3 & 423 & 32 & 2.4 & 30.9 & 27 & 340 & 35.5 & 36.5 & 23.5 & 396 & 0.60 & 1.43 & 8.5 & 12.6 & 70 \\
\hline 75 & 25.4 & 25.3 & 45.5 & 23.9 & 340 & 38.4 & 37.9 & 23.9 & 437 & 26 & 2.2 & 32.6 & 25 & 314 & 36.2 & 38.3 & 24.1 & 412 & 0.56 & 1.37 & 8.0 & 12.3 & 75 \\
\hline 80 & 26.0 & 25.8 & 47.3 & 24.2 & 314 & 38.9 & 39.7 & 24.5 & 451 & 22 & 2.0 & 34.3 & 24 & 292 & 36.9 & 40.1 & 24.7 & 428 & 0.52 & 1.32 & 7.5 & 12.0 & 80 \\
\hline 85 & 26.4 & 26.3 & 49.0 & 24.6 & 292 & 39.4 & 41.5 & 25.0 & 464 & 19 & 1.9 & 35.9 & 22 & 273 & 37.5 & 41.8 & 25.2 & 442 & 0.49 & 1.27 & 7.1 & 11.7 & 85 \\
\hline 90 & 26.8 & 26.7 & 50.5 & 25.0 & 273 & 39.9 & 43.1 & 25.5 & 476 & 16 & 1.8 & 37.5 & 21 & 257 & 38.2 & 43.5 & 25.6 & 455 & 0.47 & 1.23 & 6.7 & 11.4 & 90 \\
\hline
\end{tabular}




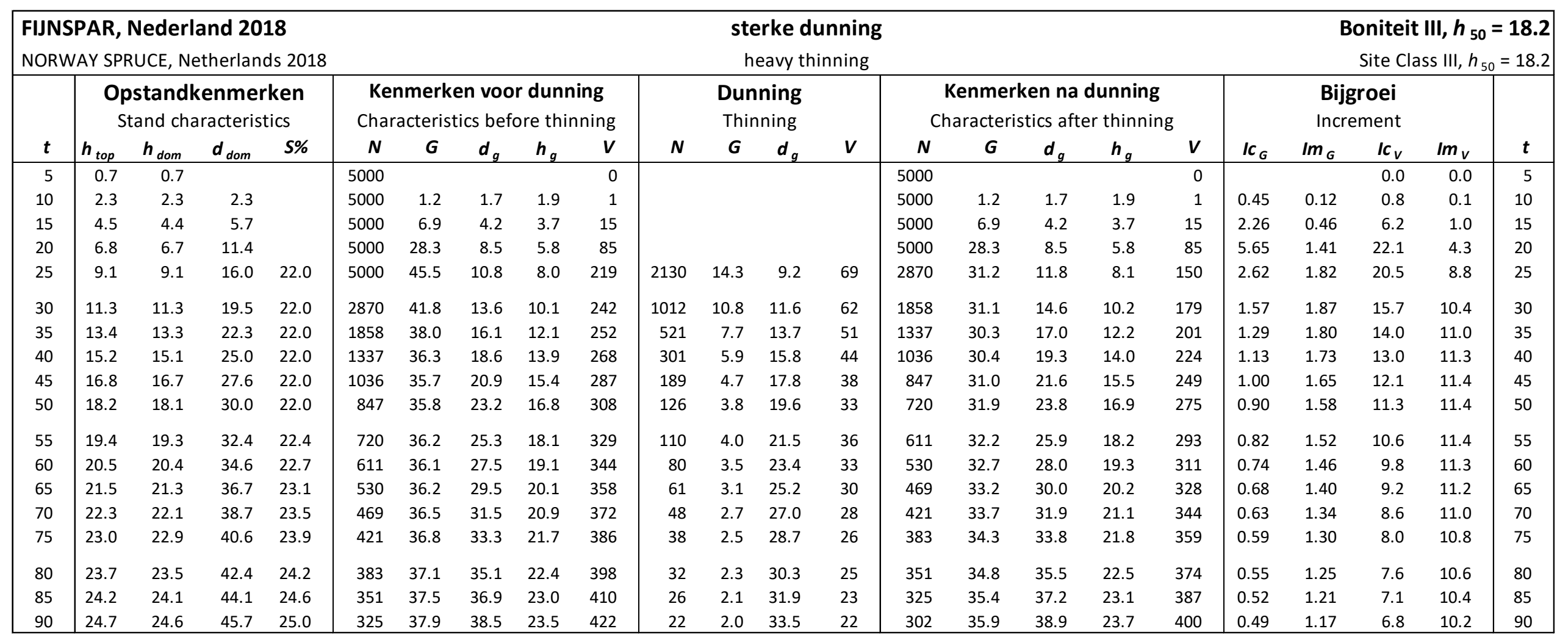




\begin{tabular}{|c|c|c|c|c|c|c|c|c|c|c|c|c|c|c|c|c|c|c|c|c|c|c|c|}
\hline \multirow{2}{*}{\multicolumn{5}{|c|}{$\begin{array}{l}\text { FIJNSPAR, Nederland } 2018 \\
\text { NORWAY SPRUCE, Netherlands } 2018 \\
\end{array}$}} & \multicolumn{15}{|c|}{ sterke dunning } & \multirow{2}{*}{\multicolumn{4}{|c|}{$\begin{array}{r}\text { Boniteit IV, } \boldsymbol{h}_{\mathbf{5 0}}=\mathbf{1 5 . 2} \\
\text { Site Class IV, } h_{50}=15.2\end{array}$}} \\
\hline & & & & & & & & & & & & eavy t & ning & & & & & & & & & & \\
\hline \multirow[b]{2}{*}{$t$} & \multicolumn{4}{|c|}{$\begin{array}{l}\text { Opstandkenmerken } \\
\text { Stand characteristics }\end{array}$} & \multicolumn{5}{|c|}{$\begin{array}{l}\text { Kenmerken voor dunning } \\
\text { Characteristics before thinning }\end{array}$} & \multicolumn{4}{|c|}{$\begin{array}{l}\text { Dunning } \\
\text { Thinning }\end{array}$} & \multicolumn{5}{|c|}{$\begin{array}{l}\text { Kenmerken na dunning } \\
\text { Characteristics after thinning }\end{array}$} & \multicolumn{4}{|c|}{$\begin{array}{c}\text { Bijgroei } \\
\text { Increment }\end{array}$} & \multirow[b]{2}{*}{$t$} \\
\hline & $\boldsymbol{h}_{\text {top }}$ & $\boldsymbol{h}_{\text {dom }}$ & $\boldsymbol{d}_{d o m}$ & S\% & $N$ & $\boldsymbol{G}$ & $d_{g}$ & $\boldsymbol{h}_{g}$ & $V$ & $N$ & $G$ & $d_{g}$ & $v$ & $N$ & $\boldsymbol{G}$ & $d_{g}$ & $h_{g}$ & $v$ & $I c_{G}$ & $I m_{G}$ & $I c_{v}$ & $I m_{v}$ & \\
\hline 5 & 0.5 & 0.5 & & & 5000 & & & & 0 & & & & & 5000 & & & & 0 & & & 0.0 & 0.0 & 5 \\
\hline 10 & 1.6 & 1.6 & 1.6 & & 5000 & 0.6 & 1.2 & 1.3 & 1 & & & & & 5000 & 0.6 & 1.2 & 1.3 & 1 & 0.17 & 0.06 & 0.2 & 0.1 & 10 \\
\hline 15 & 3.2 & 3.2 & 3.4 & & 5000 & 2.5 & 2.5 & 2.5 & 4 & & & & & 5000 & 2.5 & 2.5 & 2.5 & 4 & 0.75 & 0.17 & 1.6 & 0.3 & 15 \\
\hline 20 & 5.0 & 4.9 & 6.8 & & 5000 & 9.9 & 5.0 & 4.2 & 28 & & & & & 5000 & 9.9 & 5.0 & 4.2 & 28 & 2.44 & 0.49 & 7.2 & 1.4 & 20 \\
\hline 25 & 6.9 & 6.8 & 11.7 & & 5000 & 29.3 & 8.6 & 5.9 & 90 & & & & & 5000 & 29.3 & 8.6 & 5.9 & 90 & 4.62 & 1.17 & 18.3 & 3.6 & 25 \\
\hline 30 & 8.7 & 8.7 & 15.4 & 22.0 & 5000 & 44.1 & 10.6 & 7.7 & 205 & 1870 & 12.1 & 9.1 & 56 & 3130 & 32.0 & 11.4 & 7.7 & 148 & 2.39 & 1.47 & 17.4 & 6.8 & 30 \\
\hline 35 & 10.5 & 10.5 & 18.5 & 22.0 & 3130 & 42.3 & 13.1 & 9.4 & 230 & 980 & 9.6 & 11.2 & 52 & 2150 & 32.6 & 13.9 & 9.5 & 177 & 1.70 & 1.55 & 14.9 & 8.2 & 35 \\
\hline 40 & $\mid$\begin{tabular}{|l|}
12.2 \\
\end{tabular} & 12.1 & 20.8 & 22.0 & 2150 & 39.4 & 15.3 & 11.1 & 243 & 553 & 7.3 & 13.0 & 45 & 1597 & 32.0 & 16.0 & 11.1 & 197 & 1.19 & 1.53 & 12.2 & 8.8 & 40 \\
\hline 45 & $\mid$\begin{tabular}{|l}
13.8 \\
\end{tabular} & 13.7 & 23.1 & 22.0 & 1597 & 37.6 & 17.3 & 12.6 & 256 & 340 & 5.8 & 14.7 & 39 & 1256 & 31.9 & 18.0 & 12.7 & 217 & 1.06 & 1.48 & 11.5 & 9.1 & 45 \\
\hline 50 & 15.2 & 15.1 & 25.3 & 22.0 & 1256 & 36.9 & 19.3 & 14.0 & 273 & 224 & 4.7 & 16.4 & 35 & 1033 & 32.2 & 19.9 & 14.1 & 238 & 0.95 & 1.43 & 10.9 & 9.3 & 50 \\
\hline 55 & 16.5 & 16.4 & 27.5 & 22.4 & 1033 & 36.7 & 21.3 & 15.3 & 291 & 183 & 4.7 & 18.1 & 37 & 849 & 32.0 & 21.9 & 15.4 & 253 & 0.86 & 1.39 & 10.2 & 9.4 & 55 \\
\hline 60 & 17.6 & 17.5 & 29.7 & 22.7 & 849 & 36.1 & 23.3 & 16.4 & 303 & 132 & 4.1 & 19.9 & 34 & 718 & 32.0 & 23.8 & 16.5 & 268 & 0.79 & 1.34 & 9.6 & 9.5 & 60 \\
\hline 65 & $\mid 18.7$ & 18.5 & 31.7 & 23.1 & 718 & 35.8 & 25.2 & 17.5 & 315 & 98 & 3.6 & 21.6 & 32 & 620 & 32.2 & 25.7 & 17.6 & 283 & 0.72 & 1.29 & 9.0 & 9.5 & 65 \\
\hline 70 & $\mid 19.6$ & 19.5 & 33.7 & 23.5 & 620 & 35.7 & 27.1 & 18.4 & 327 & 75 & 3.2 & 23.2 & 29 & 545 & 32.5 & 27.6 & 18.5 & 298 & 0.67 & 1.25 & 8.5 & 9.4 & 70 \\
\hline 75 & 20.4 & 20.3 & 35.5 & 23.9 & 545 & 35.7 & 28.9 & 19.3 & 339 & 59 & 2.9 & 24.9 & 27 & 486 & 32.9 & 29.3 & 19.4 & 311 & 0.62 & 1.21 & 8.0 & 9.3 & 75 \\
\hline 80 & 21.2 & 21.0 & 37.3 & 24.2 & 486 & 35.8 & 30.6 & 20.0 & 350 & 47 & 2.6 & 26.5 & 26 & 438 & 33.2 & 31.1 & 20.2 & 325 & 0.58 & 1.17 & 7.6 & 9.2 & 80 \\
\hline 85 & 21.9 & 21.7 & 39.1 & 24.6 & 438 & 36.0 & 32.3 & 20.7 & 361 & 39 & 2.4 & 28.0 & 24 & 400 & 33.6 & 32.7 & 20.9 & 337 & 0.54 & 1.14 & 7.2 & 9.1 & 85 \\
\hline 90 & 22.5 & 22.3 & 40.7 & 25.0 & 400 & 36.2 & 34.0 & 21.4 & 372 & 32 & 2.2 & 29.6 & 23 & 367 & 34.0 & 34.4 & 21.5 & 349 & 0.51 & 1.10 & 6.8 & 9.0 & 90 \\
\hline
\end{tabular}




\begin{tabular}{|c|c|c|c|c|c|c|c|c|c|c|c|c|c|c|c|c|c|c|c|c|c|c|c|}
\hline \multirow{2}{*}{\multicolumn{5}{|c|}{$\begin{array}{l}\text { FIJNSPAR, Nederland } 2018 \\
\text { NORWAY SPRUCE, Netherlands } 2018 \\
\end{array}$}} & \multicolumn{15}{|c|}{ sterke dunning } & \multirow{2}{*}{\multicolumn{4}{|c|}{$\begin{array}{r}\text { Boniteit V, } \boldsymbol{h}_{\mathbf{5 0}}=\mathbf{1 2 . 2} \\
\text { Site Class V, } h_{50}=12.2\end{array}$}} \\
\hline & & & & & & & & & & & & eavy t & ning & & & & & & & & & & \\
\hline \multirow[b]{2}{*}{$t$} & \multicolumn{4}{|c|}{$\begin{array}{l}\text { Opstandkenmerken } \\
\text { Stand characteristics }\end{array}$} & \multicolumn{5}{|c|}{$\begin{array}{l}\text { Kenmerken voor dunning } \\
\text { Characteristics before thinning }\end{array}$} & \multicolumn{4}{|c|}{$\begin{array}{l}\text { Dunning } \\
\text { Thinning }\end{array}$} & \multicolumn{5}{|c|}{$\begin{array}{l}\text { Kenmerken na dunning } \\
\text { Characteristics after thinning }\end{array}$} & \multicolumn{4}{|c|}{$\begin{array}{c}\text { Bijgroei } \\
\text { Increment }\end{array}$} & \multirow[b]{2}{*}{$t$} \\
\hline & $\boldsymbol{h}_{\text {top }}$ & $\boldsymbol{h}_{\text {dom }}$ & $\boldsymbol{d}_{d o m}$ & S\% & $N$ & $\boldsymbol{G}$ & $d_{g}$ & $h_{g}$ & $V$ & $N$ & $G$ & $d_{g}$ & $v$ & $N$ & $\boldsymbol{G}$ & $d_{g}$ & $h_{g}$ & $v$ & $I c_{G}$ & $I m_{G}$ & $I c_{v}$ & $I m_{V}$ & \\
\hline 5 & 0.3 & 0.3 & & & 5000 & & & & 0 & & & & & 5000 & & & & 0 & & & 0.0 & 0.0 & 5 \\
\hline 10 & 1.1 & 1.1 & & & 5000 & & & & 0 & & & & & 5000 & & & & 0 & & & 0.2 & 0.0 & 10 \\
\hline 15 & 2.2 & 2.2 & 2.2 & & 5000 & 1.0 & 1.6 & 1.8 & 1 & & & & & 5000 & 1.0 & 1.6 & 1.8 & 1 & 0.26 & 0.07 & 0.4 & 0.1 & 15 \\
\hline 20 & 3.5 & 3.5 & 4.0 & & 5000 & 3.4 & 3.0 & 2.9 & 7 & & & & & 5000 & 3.4 & 3.0 & 2.9 & 7 & 0.79 & 0.17 & 1.8 & 0.4 & 20 \\
\hline 25 & 5.0 & 4.9 & 6.8 & & 5000 & 9.9 & 5.0 & 4.2 & 24 & & & & & 5000 & 9.9 & 5.0 & 4.2 & 24 & 1.95 & 0.40 & 5.8 & 0.9 & 25 \\
\hline 30 & 6.5 & 6.4 & 10.6 & & 5000 & 24.2 & 7.8 & 5.6 & 71 & & & & & 5000 & 24.2 & 7.8 & 5.6 & 71 & 3.87 & 0.81 & 14.0 & 2.4 & 30 \\
\hline 35 & 8.0 & 7.9 & 14.1 & 22.0 & 5000 & 39.6 & 10.0 & 7.0 & 171 & 1260 & 7.2 & 8.5 & 31 & 3740 & 32.4 & 10.5 & 7.0 & 139 & 2.30 & 1.13 & 14.8 & 4.9 & 35 \\
\hline 40 & 9.5 & 9.4 & 16.8 & 22.0 & 3740 & 42.6 & 12.0 & 8.4 & 212 & 1073 & 8.9 & 10.3 & 44 & 2667 & 33.8 & 12.7 & 8.5 & 168 & 1.81 & 1.25 & 13.9 & 6.1 & 40 \\
\hline 45 & $\mid$\begin{tabular}{|l|}
10.9 \\
\end{tabular} & 10.8 & 19.2 & 22.0 & 2667 & 41.6 & 14.1 & 9.8 & 232 & 647 & 7.3 & 12.0 & 41 & 2020 & 34.3 & 14.7 & 9.9 & 191 & 1.29 & 1.28 & 11.7 & 6.8 & 45 \\
\hline 50 & 12.2 & 12.1 & 20.9 & 22.0 & 2020 & 39.7 & 15.8 & 11.1 & 244 & 417 & 5.9 & 13.4 & 36 & 1603 & 33.8 & 16.4 & 11.2 & 208 & 1.01 & 1.26 & 10.2 & 7.2 & 50 \\
\hline 55 & 13.4 & 13.4 & 22.9 & 22.4 & 1603 & 38.6 & 17.5 & 12.3 & 258 & 327 & 5.7 & 14.9 & 38 & 1276 & 32.9 & 18.1 & 12.4 & 219 & 0.91 & 1.23 & 9.7 & 7.5 & 55 \\
\hline 60 & 14.6 & 14.5 & 24.8 & 22.7 & 1276 & 37.3 & 19.3 & 13.5 & 266 & 229 & 4.9 & 16.5 & 35 & 1047 & 32.4 & 19.8 & 13.6 & 231 & 0.83 & 1.20 & 9.2 & 7.6 & 60 \\
\hline 65 & $\mid 15.7$ & 15.6 & 26.7 & 23.1 & 1047 & 36.4 & 21.0 & 14.6 & 276 & 167 & 4.3 & 18.0 & 32 & 880 & 32.1 & 21.6 & 14.7 & 244 & 0.76 & 1.17 & 8.7 & 7.7 & 65 \\
\hline 70 & $\mid 16.7$ & 16.5 & 28.5 & 23.5 & 880 & 35.8 & 22.8 & 15.6 & 286 & 125 & 3.8 & 19.5 & 30 & 755 & 32.0 & 23.2 & 15.7 & 256 & 0.71 & 1.14 & 8.2 & 7.8 & 70 \\
\hline 75 & 17.6 & 17.4 & 30.3 & 23.9 & 755 & 35.4 & 24.4 & 16.5 & 296 & 97 & 3.4 & 21.1 & 28 & 658 & 32.1 & 24.9 & 16.6 & 267 & 0.65 & 1.11 & 7.8 & 7.8 & 75 \\
\hline 80 & 18.4 & 18.3 & 32.1 & 24.2 & 658 & 35.2 & 26.1 & 17.4 & 305 & 76 & 3.0 & 22.6 & 26 & 582 & 32.2 & 26.5 & 17.5 & 279 & 0.61 & 1.08 & 7.4 & 7.8 & 80 \\
\hline 85 & $\mid$\begin{tabular}{|l|}
19.1 \\
\end{tabular} & 19.0 & 33.8 & 24.6 & 582 & 35.1 & 27.7 & 18.1 & 315 & 61 & 2.8 & 24.0 & 25 & 521 & 32.3 & 28.1 & 18.3 & 290 & 0.57 & 1.05 & 7.1 & 7.7 & 85 \\
\hline 90 & 19.8 & 19.7 & 35.4 & 25.0 & 521 & 35.1 & 29.3 & 18.9 & 325 & 50 & 2.6 & 25.5 & 24 & 471 & 32.6 & 29.7 & 19.0 & 301 & 0.54 & 1.02 & 6.7 & 7.7 & 90 \\
\hline
\end{tabular}




\section{Zomereik (Quercus robur)}

Jansen, J.J., A. Oosterbaan, G.M.J. Mohren en J. den Ouden

Bron: Jansen, J.J., A. Oosterbaan, G.M.J. Mohren en J. den Ouden, 2018. Groei en productie van zomereik in Nederland. FEM Groei en Productie Rapport $2018-4,87 \mathrm{blz}$.

Dit rapport is gratis te downloaden op:

https://doi.org/10.18174/444093

\section{Common oak}

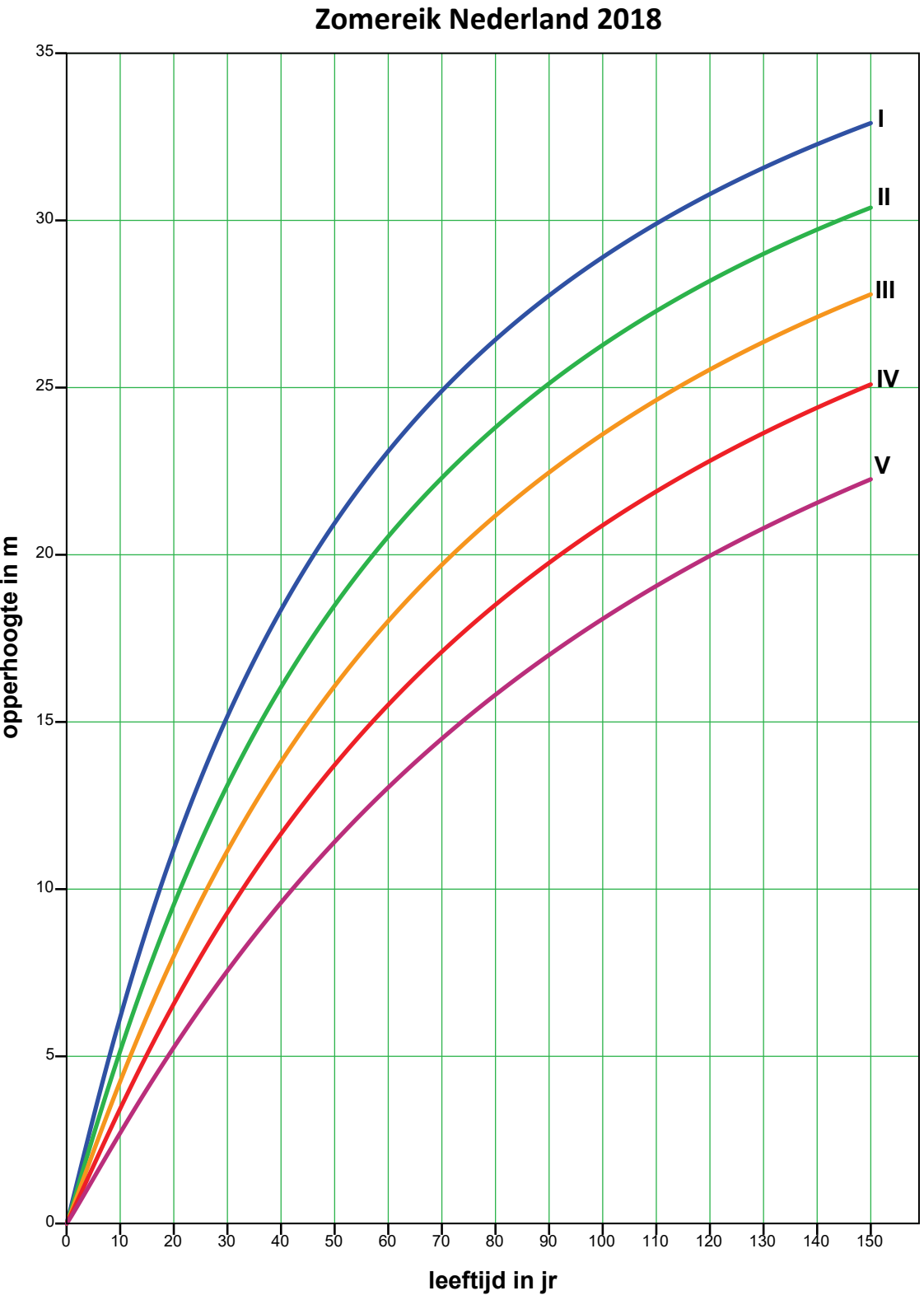




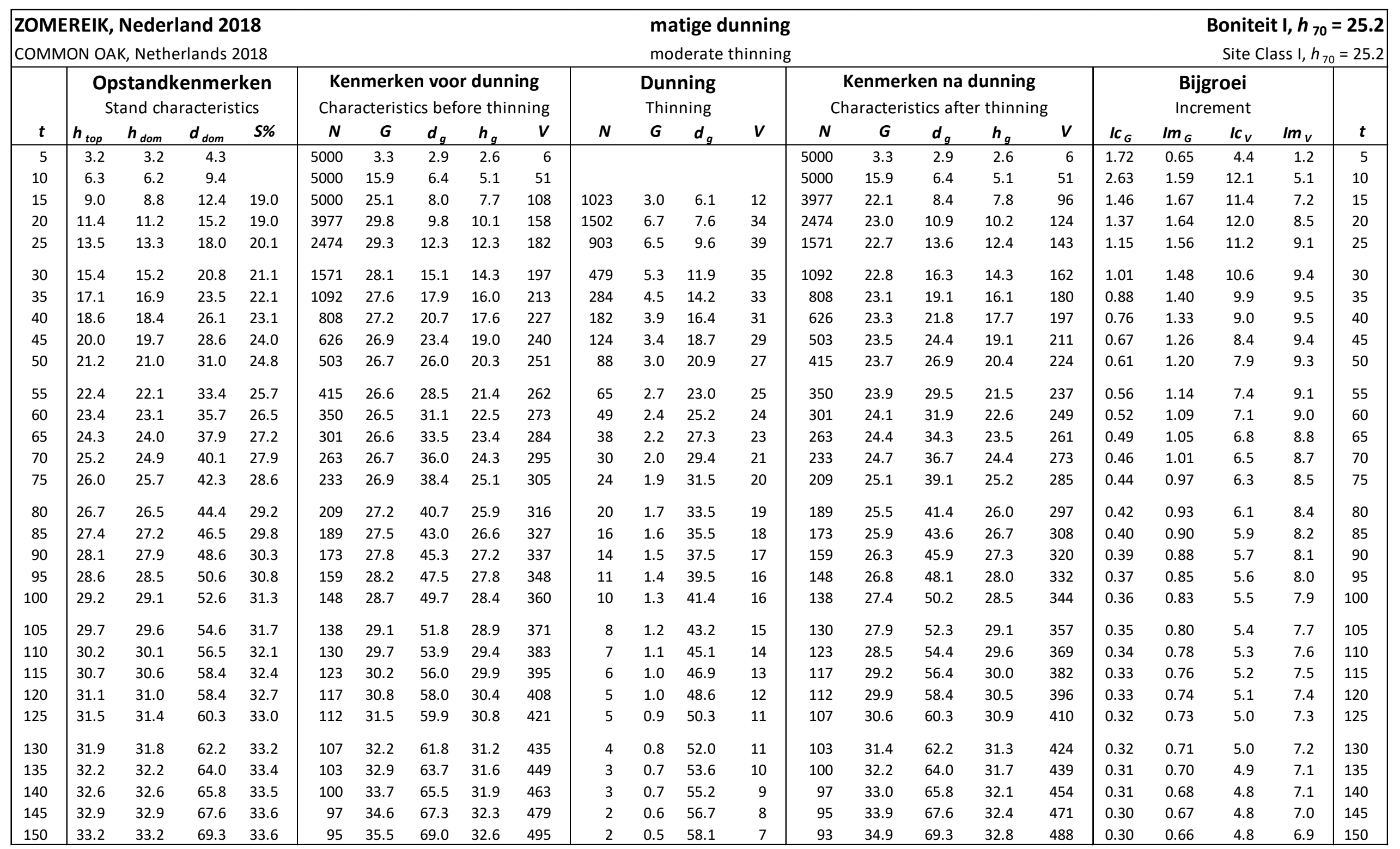




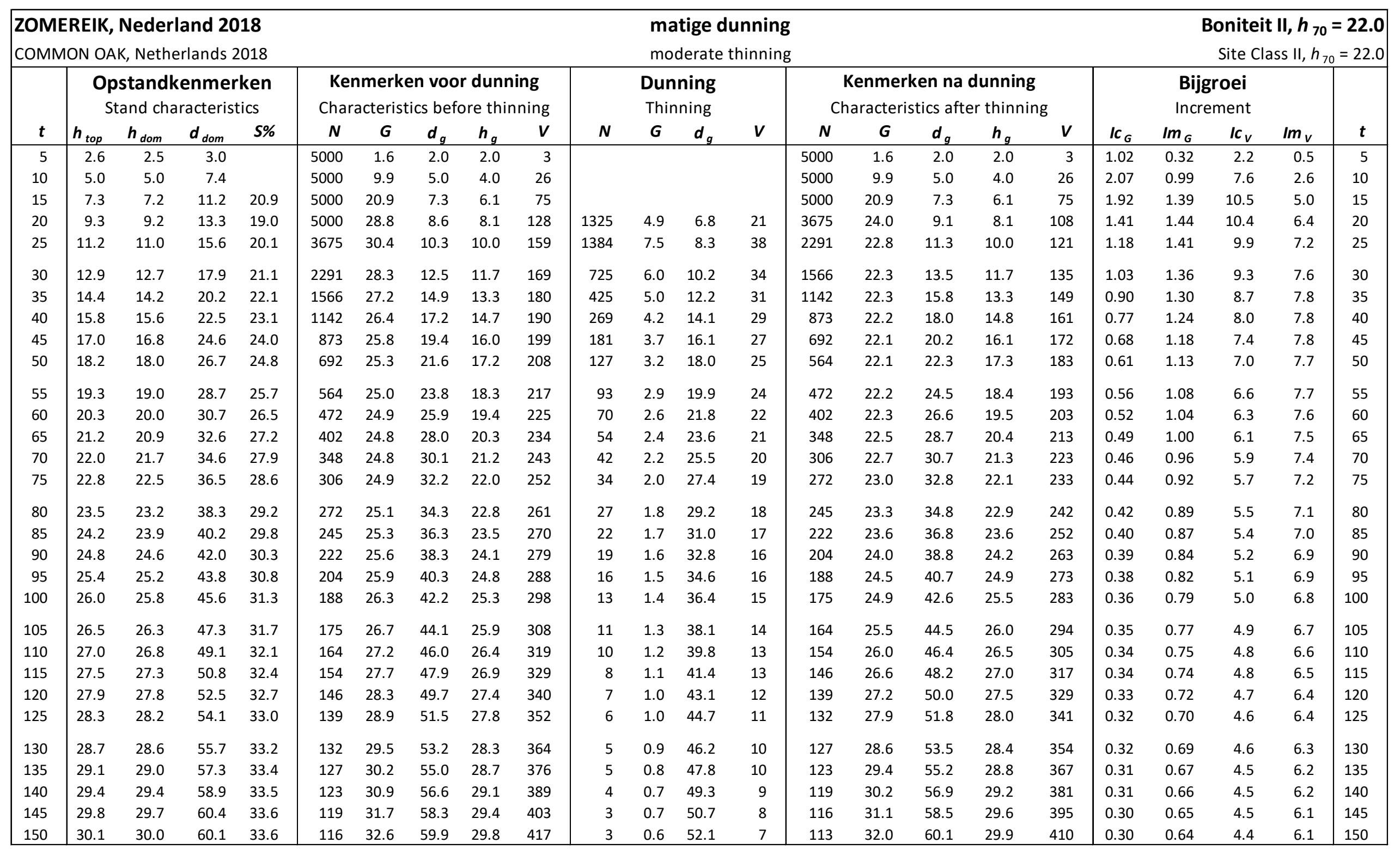




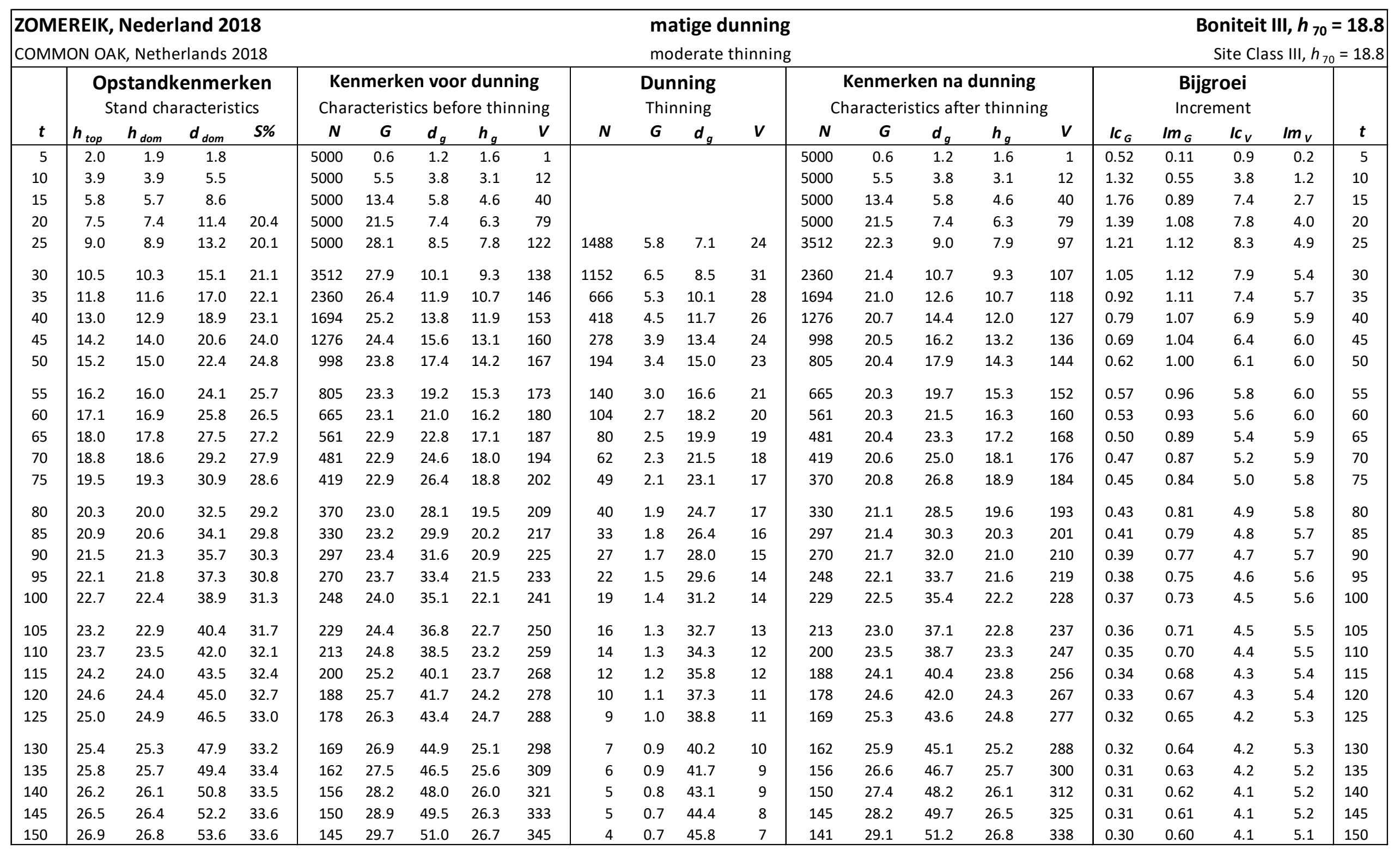




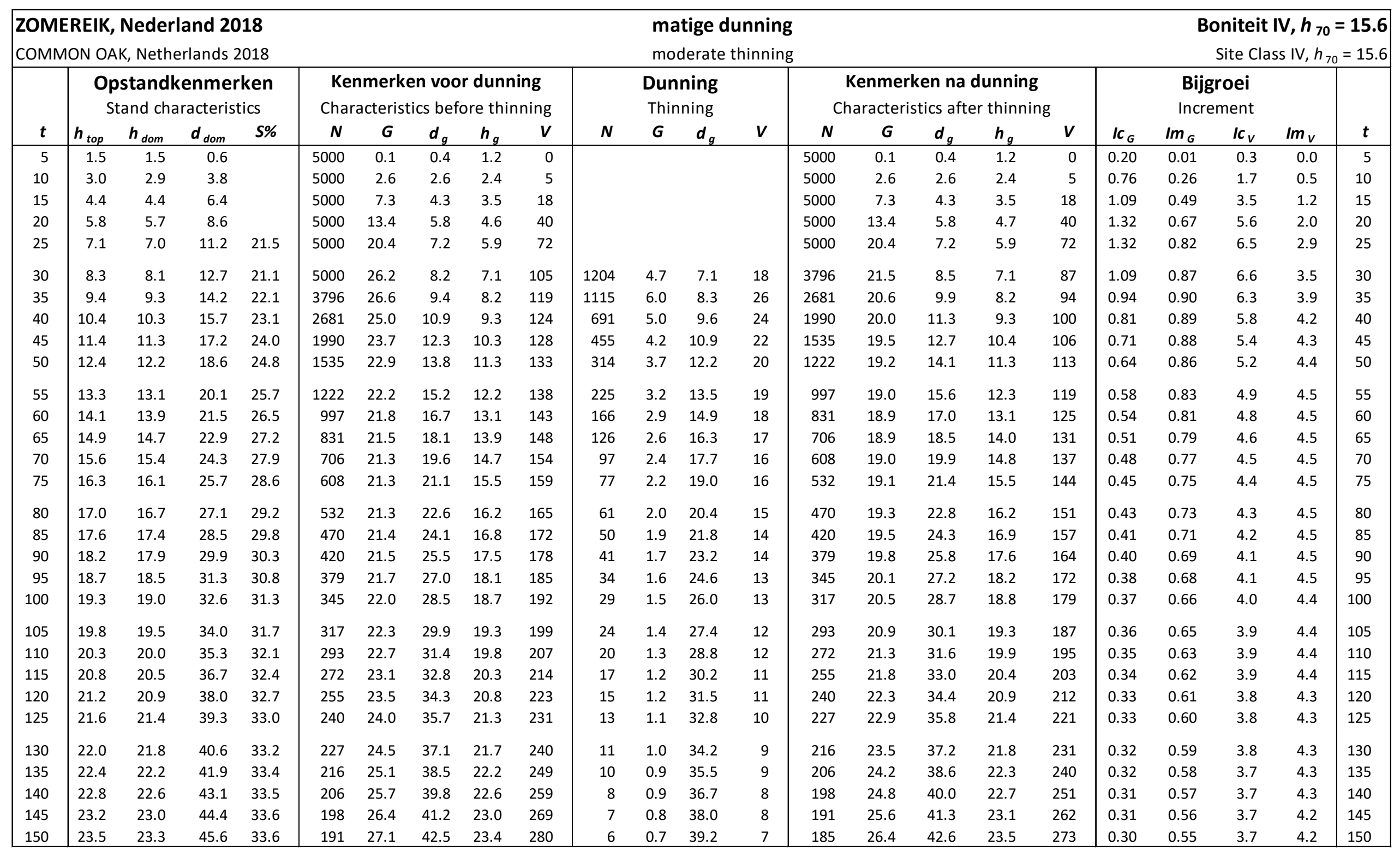




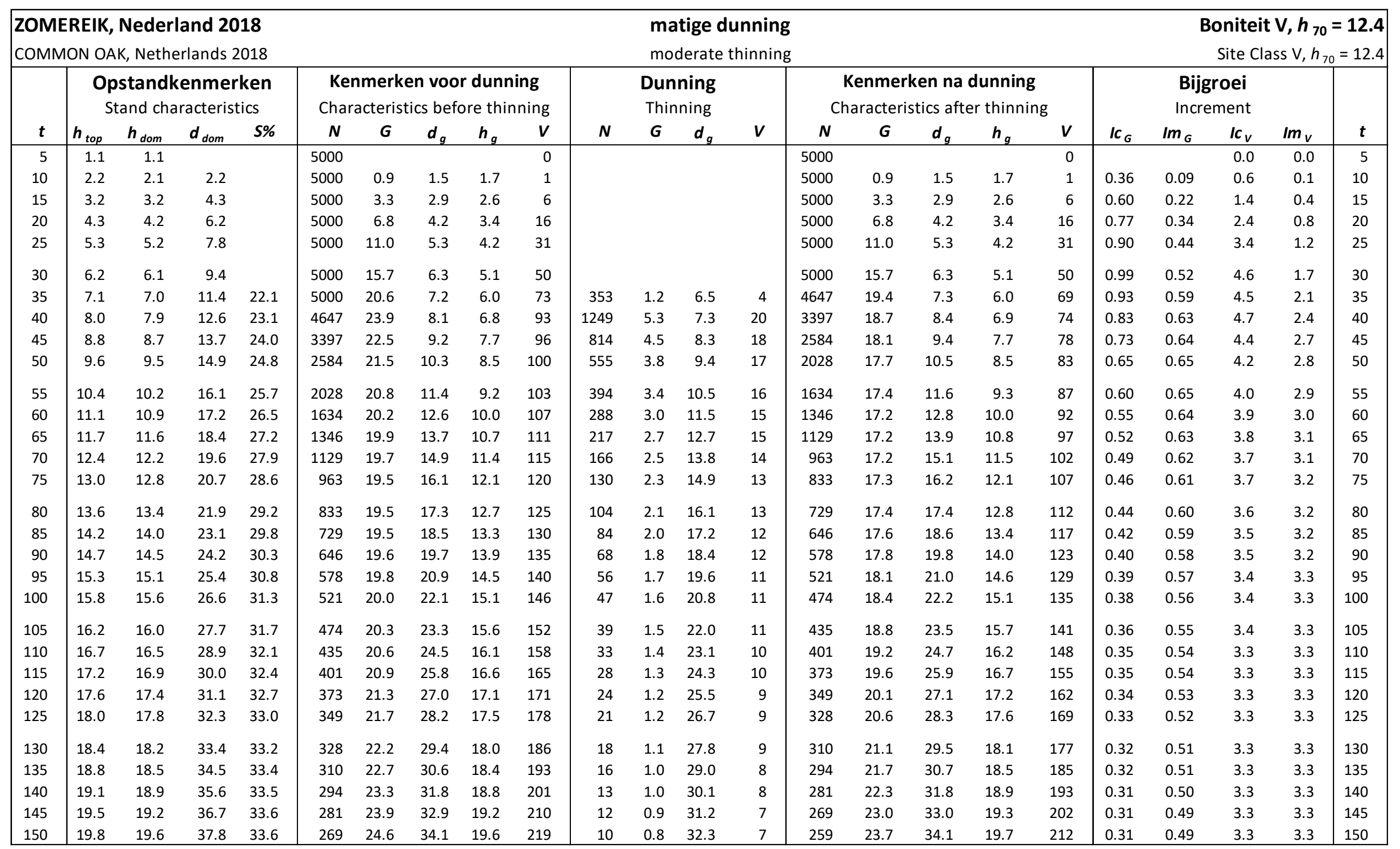




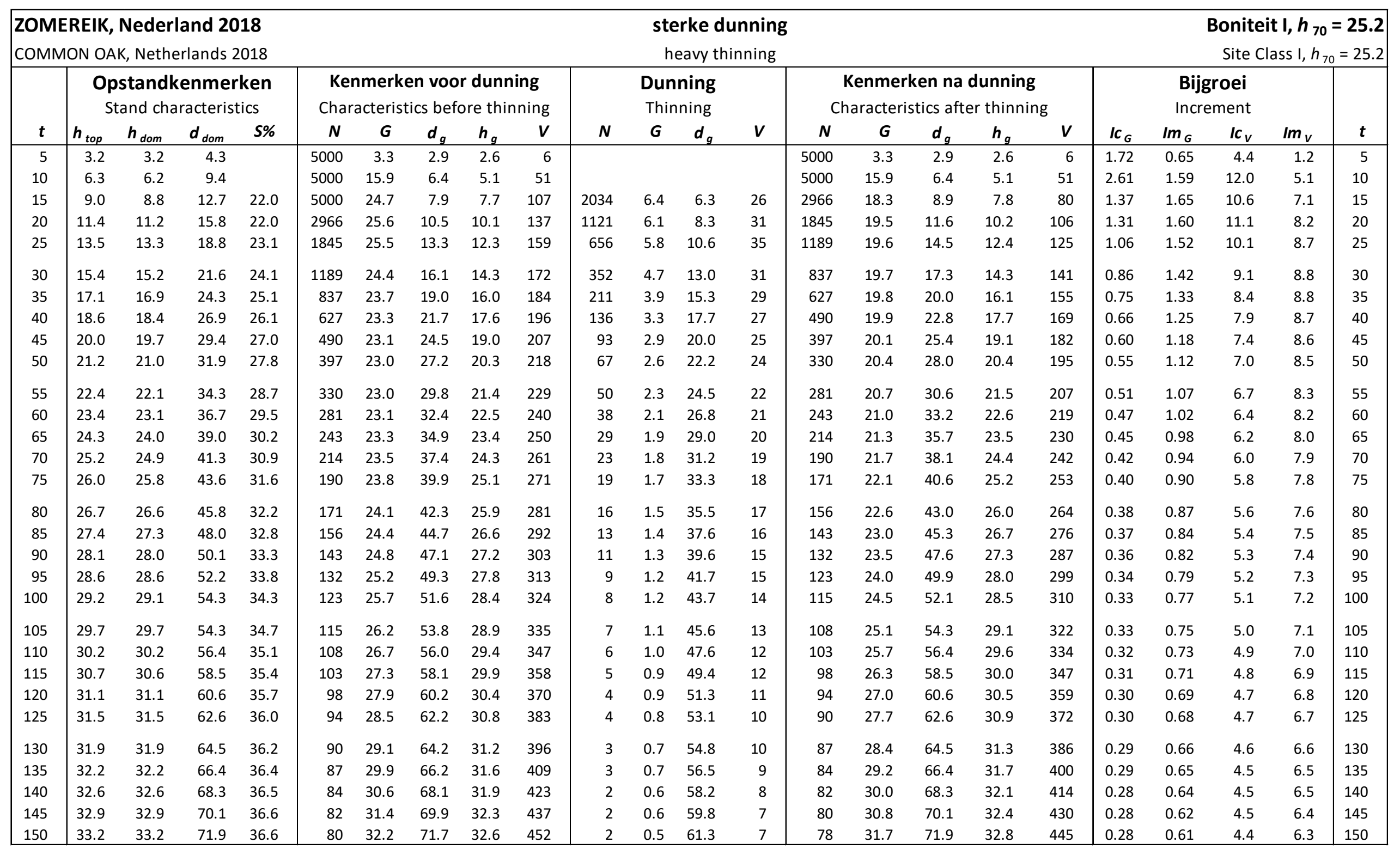




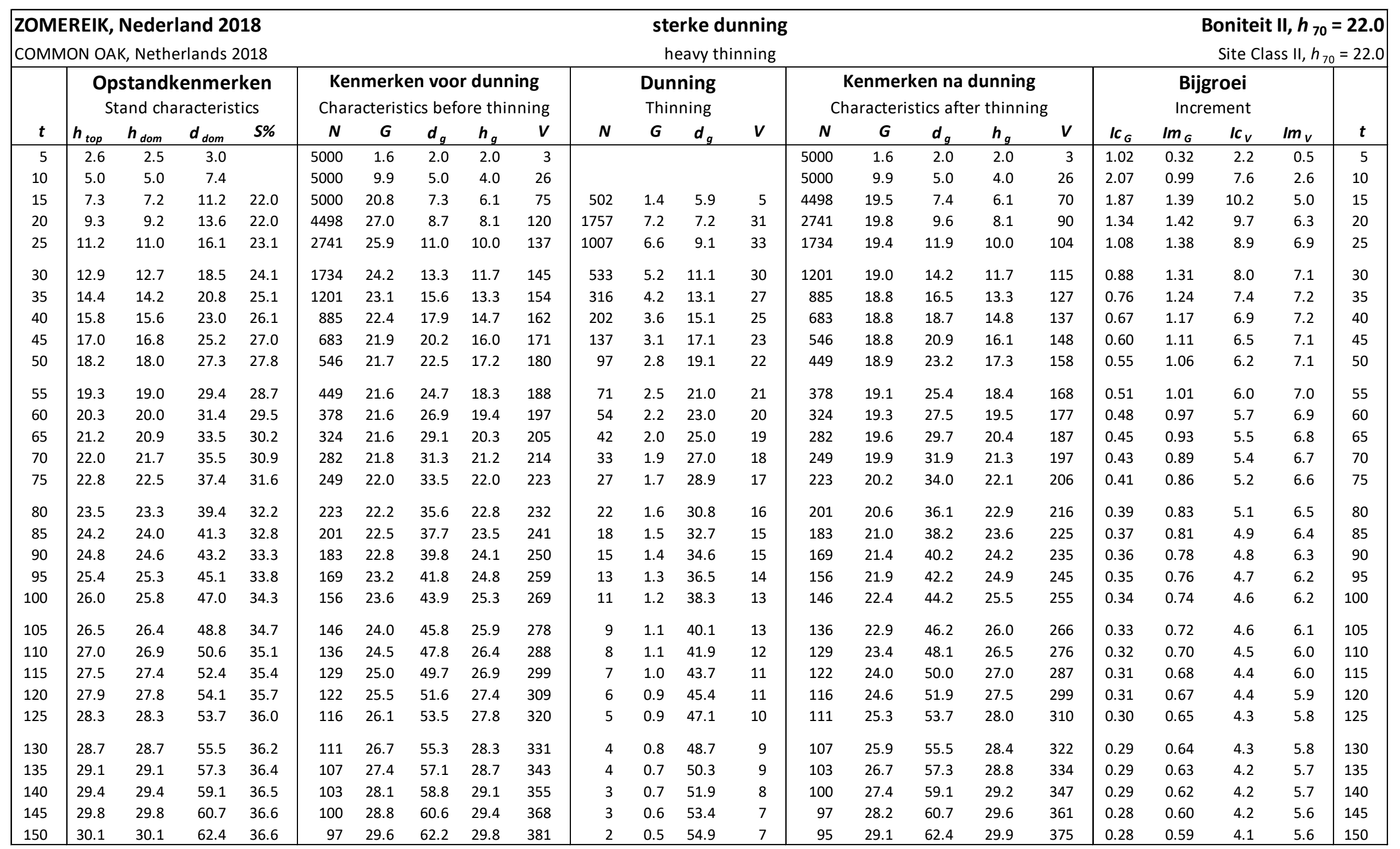




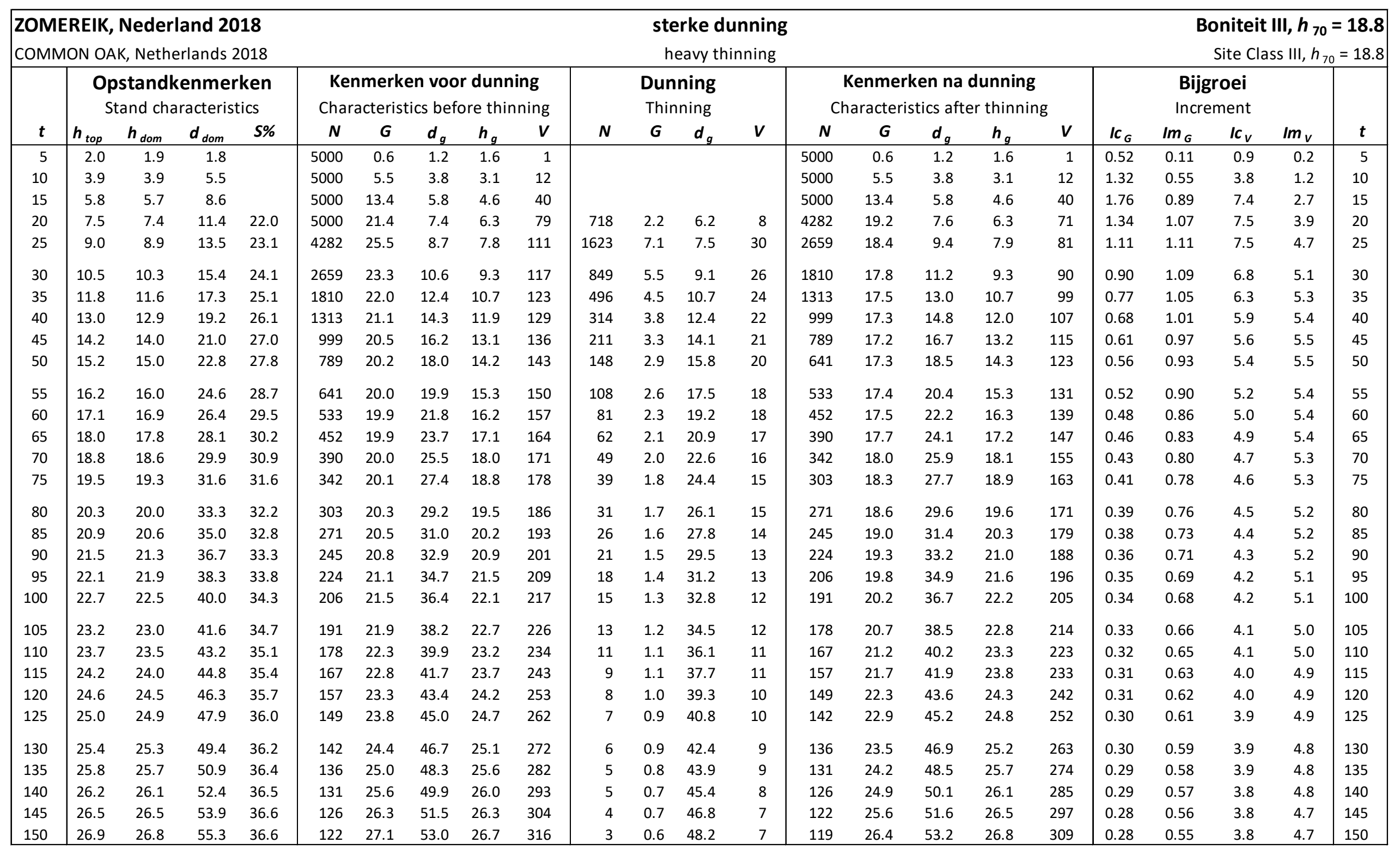




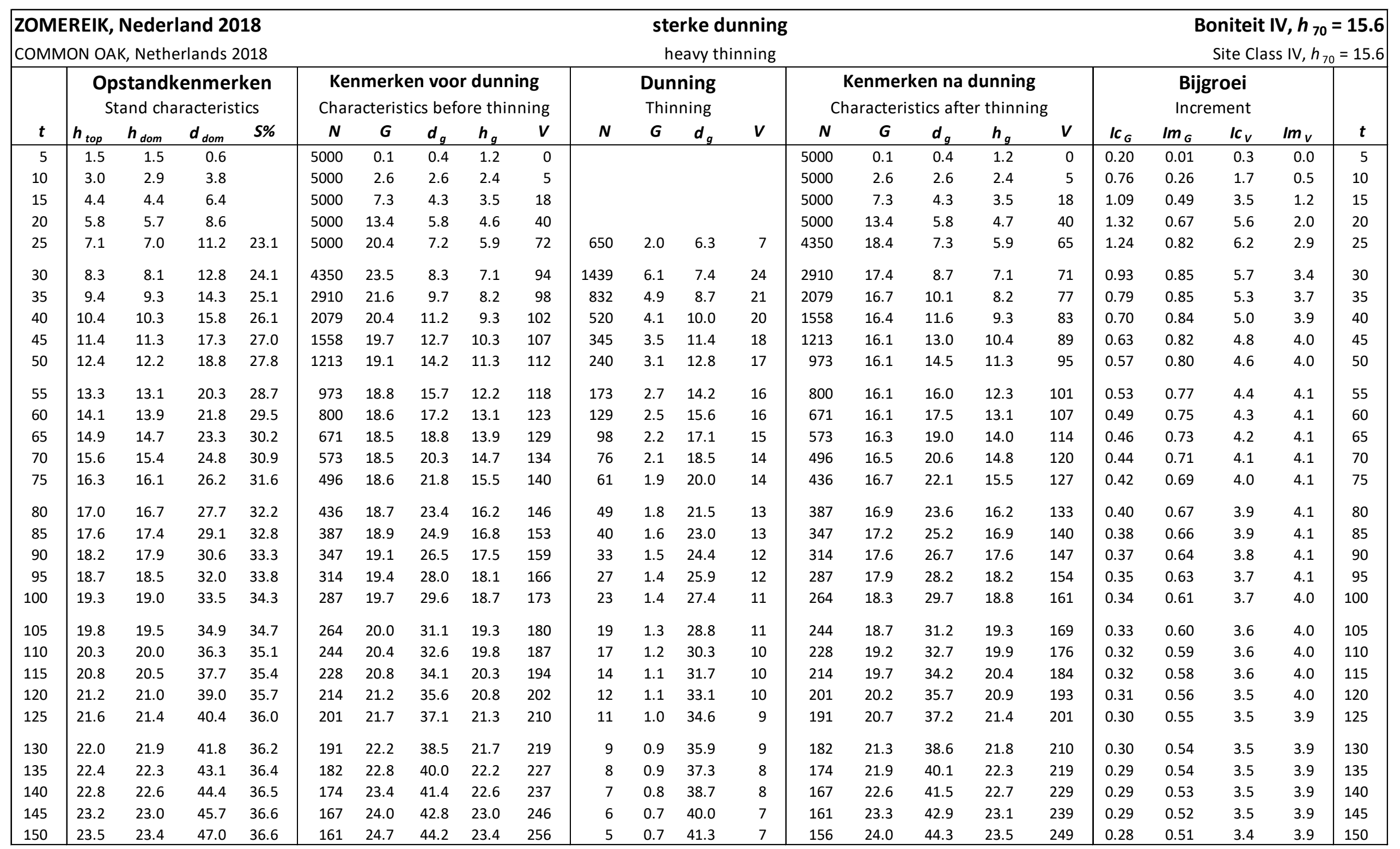




\begin{tabular}{|c|c|c|c|c|c|c|c|c|c|c|c|c|c|c|c|c|c|c|c|c|c|c|c|}
\hline \multicolumn{5}{|c|}{$\begin{array}{l}\text { ZOMEREIK, Nederland } 2018 \\
\text { COMMON OAK, Netherlands } 2018\end{array}$} & \multicolumn{15}{|c|}{ sterke dunning } & \multicolumn{4}{|c|}{ Boniteit V, $h_{70}=12.4$} \\
\hline \multirow[b]{2}{*}{$t$} & \multicolumn{4}{|c|}{$\begin{array}{l}\text { Opstandkenmerken } \\
\text { Stand characteristics }\end{array}$} & \multicolumn{5}{|c|}{$\begin{array}{l}\text { Kenmerken voor dunning } \\
\text { Characteristics before thinning }\end{array}$} & \multicolumn{4}{|c|}{$\begin{array}{c}\text { Dunning } \\
\text { Thinning }\end{array}$} & \multicolumn{5}{|c|}{$\begin{array}{l}\text { Kenmerken na dunning } \\
\text { Characteristics after thinning }\end{array}$} & \multicolumn{4}{|c|}{$\begin{array}{l}\text { Bijgroei } \\
\text { Increment }\end{array}$} & \multirow[b]{2}{*}{$t$} \\
\hline & $h_{\text {top }}$ & $\boldsymbol{h}_{\text {dom }}$ & $\boldsymbol{d}_{\text {dom }}$ & $S \%$ & $N$ & $\boldsymbol{G}$ & $d_{g}$ & $\boldsymbol{h}_{g}$ & $\boldsymbol{V}$ & $N$ & $\boldsymbol{G}$ & $d_{g}$ & $v$ & $N$ & $\boldsymbol{G}$ & $d_{g}$ & $\boldsymbol{h}_{g}$ & $v$ & $I c_{G}$ & $I m_{G}$ & $I c_{v}$ & $I m_{V}$ & \\
\hline 5 & 1.1 & 1.1 & & & 5000 & & & & 0 & & & & & 5000 & & & & 0 & & & 0.0 & 0.0 & 5 \\
\hline 10 & 2.2 & 2.1 & 2.2 & & 5000 & 0.9 & 1.5 & 1.7 & 1 & & & & & 5000 & 0.9 & 1.5 & 1.7 & 1 & 0.36 & 0.09 & 0.6 & 0.1 & 10 \\
\hline 15 & 3.2 & 3.2 & 4.3 & & 5000 & 3.3 & 2.9 & 2.6 & 6 & & & & & 5000 & 3.3 & 2.9 & 2.6 & 6 & 0.60 & 0.22 & 1.4 & 0.4 & 15 \\
\hline 20 & 4.3 & 4.2 & 6.2 & & 5000 & 6.8 & 4.2 & 3.4 & 16 & & & & & 5000 & 6.8 & 4.2 & 3.4 & 16 & 0.77 & 0.34 & 2.4 & 0.8 & 20 \\
\hline 25 & 5.3 & 5.2 & 7.8 & & 5000 & 11.0 & 5.3 & 4.2 & 31 & & & & & 5000 & 11.0 & 5.3 & 4.2 & 31 & 0.90 & 0.44 & 3.4 & 1.2 & 25 \\
\hline 30 & 6.2 & 6.1 & 9.4 & & 5000 & 15.7 & 6.3 & 5.1 & 50 & & & & & 5000 & 15.7 & 6.3 & 5.1 & 50 & 0.99 & 0.52 & 4.6 & 1.7 & 30 \\
\hline 35 & 7.1 & 7.0 & 11.4 & 25.1 & 5000 & 20.6 & 7.2 & 6.0 & 73 & 1397 & 4.8 & 6.6 & 16 & 3603 & 15.7 & 7.5 & 6.0 & 56 & 0.86 & 0.59 & 4.1 & 2.1 & 35 \\
\hline 40 & 8.0 & 7.9 & 12.6 & 26.1 & 3603 & 19.6 & 8.3 & 6.8 & 77 & 942 & 4.3 & 7.7 & 16 & 2660 & 15.3 & 8.5 & 6.9 & 61 & 0.72 & 0.61 & 4.0 & 2.3 & 40 \\
\hline 45 & 8.8 & 8.7 & 13.8 & 27.0 & 2660 & 18.7 & 9.5 & 7.7 & 81 & 619 & 3.7 & 8.7 & 15 & 2041 & 15.0 & 9.7 & 7.7 & 65 & 0.65 & 0.62 & 3.9 & 2.5 & 45 \\
\hline 50 & 9.6 & 9.5 & 15.0 & 27.8 & 2041 & 18.0 & 10.6 & 8.5 & 84 & 426 & 3.2 & 9.8 & 14 & 1615 & 14.8 & 10.8 & 8.5 & 70 & 0.59 & 0.62 & 3.7 & 2.6 & 50 \\
\hline 55 & 10.4 & 10.2 & 16.2 & 28.7 & 1615 & 17.6 & 11.8 & 9.2 & 88 & 305 & 2.9 & 11.0 & 14 & 1310 & 14.7 & 12.0 & 9.3 & 75 & 0.54 & 0.61 & 3.6 & 2.7 & 55 \\
\hline 60 & 11.1 & 10.9 & 17.5 & 29.5 & 1310 & 17.4 & 13.0 & 10.0 & 92 & 224 & 2.6 & 12.1 & 13 & 1086 & 14.8 & 13.2 & 10.0 & 79 & 0.50 & 0.61 & 3.5 & 2.8 & 60 \\
\hline 65 & 11.7 & 11.6 & 18.7 & 30.2 & 1086 & 17.2 & 14.2 & 10.7 & 97 & 170 & 2.4 & 13.3 & 13 & 916 & 14.8 & 14.4 & 10.8 & 84 & 0.47 & 0.60 & 3.5 & 2.9 & 65 \\
\hline 70 & 12.4 & 12.2 & 19.9 & 30.9 & 916 & 17.1 & 15.4 & 11.4 & 101 & 131 & 2.2 & 14.5 & 12 & 785 & 15.0 & 15.6 & 11.5 & 89 & 0.45 & 0.59 & 3.4 & 2.9 & 70 \\
\hline 75 & 13.0 & 12.8 & 21.1 & 31.6 & 785 & 17.1 & 16.7 & 12.1 & 106 & 103 & 2.0 & 15.7 & 12 & 682 & 15.2 & 16.8 & 12.1 & 94 & 0.42 & 0.58 & 3.3 & 2.9 & 75 \\
\hline 80 & 13.6 & 13.4 & 22.3 & 32.2 & 682 & 17.2 & 17.9 & 12.7 & 111 & 82 & 1.9 & 16.9 & 11 & 600 & 15.4 & 18.1 & 12.8 & 99 & 0.40 & 0.57 & 3.3 & 3.0 & 80 \\
\hline 85 & 14.2 & 14.0 & 23.6 & 32.8 & 600 & 17.3 & 19.2 & 13.3 & 116 & 67 & 1.7 & 18.1 & 11 & 533 & 15.6 & 19.3 & 13.4 & 105 & 0.39 & 0.56 & 3.3 & 3.0 & 85 \\
\hline 90 & $\mid$\begin{tabular}{|l|}
14.7 \\
\end{tabular} & 14.5 & 24.8 & 33.3 & 533 & 17.5 & 20.5 & 13.9 & 121 & 55 & 1.6 & 19.4 & 11 & 478 & 15.9 & 20.6 & 14.0 & 110 & 0.37 & 0.55 & 3.2 & 3.0 & 90 \\
\hline 95 & 15.3 & 15.1 & 26.0 & 33.8 & 478 & 17.7 & 21.7 & 14.5 & 126 & 45 & 1.5 & 20.6 & 10 & 433 & 16.2 & 21.8 & 14.6 & 116 & 0.36 & 0.54 & 3.2 & 3.0 & 95 \\
\hline 100 & 15.8 & 15.6 & 27.2 & 34.3 & 433 & 18.0 & 23.0 & 15.1 & 132 & 38 & 1.4 & 21.9 & 10 & 395 & 16.5 & 23.1 & 15.1 & 122 & 0.35 & 0.53 & 3.1 & 3.0 & 100 \\
\hline 105 & 16.2 & 16.0 & 28.4 & 34.7 & 395 & 18.3 & 24.3 & 15.6 & 138 & 32 & 1.3 & 23.1 & 10 & 363 & 16.9 & 24.4 & 15.7 & 128 & 0.34 & 0.52 & 3.1 & 3.0 & 105 \\
\hline 110 & 16.7 & 16.5 & 29.6 & 35.1 & 363 & 18.6 & 25.5 & 16.1 & 143 & 27 & 1.3 & 24.4 & 9 & 336 & 17.3 & 25.6 & 16.2 & 134 & 0.33 & 0.51 & 3.1 & 3.0 & 110 \\
\hline 115 & 17.2 & 16.9 & 30.8 & 35.4 & 336 & 18.9 & 26.8 & 16.6 & 150 & 23 & 1.2 & 25.6 & 9 & 312 & 17.7 & 26.9 & 16.7 & 141 & 0.32 & 0.50 & 3.1 & 3.0 & 115 \\
\hline 120 & 17.6 & 17.4 & 32.0 & 35.7 & 312 & 19.3 & 28.1 & 17.1 & 156 & 20 & 1.1 & 26.8 & 9 & 293 & 18.2 & 28.1 & 17.2 & 147 & 0.31 & 0.49 & 3.1 & 3.0 & 120 \\
\hline 125 & 18.0 & 17.8 & 33.2 & 36.0 & 293 & 19.7 & 29.3 & 17.5 & 163 & 17 & 1.1 & 28.1 & 8 & 275 & 18.7 & 29.4 & 17.6 & 154 & 0.31 & 0.49 & 3.1 & 3.0 & 125 \\
\hline 130 & 18.4 & 18.2 & 34.3 & 36.2 & 275 & 20.2 & 30.6 & 18.0 & 170 & 15 & 1.0 & 29.3 & 8 & 261 & 19.2 & 30.6 & 18.1 & 162 & 0.30 & 0.48 & 3.0 & 3.0 & 130 \\
\hline 135 & 18.8 & 18.5 & 35.5 & 36.4 & 261 & 20.7 & 31.8 & 18.4 & 177 & 13 & 0.9 & 30.5 & 8 & 248 & 19.8 & 31.9 & 18.5 & 169 & 0.30 & 0.47 & 3.0 & 3.0 & 135 \\
\hline 140 & $\mid$\begin{tabular}{|l|}
19.1 \\
\end{tabular} & 18.9 & 36.7 & 36.5 & 248 & 21.2 & 33.0 & 18.8 & 184 & 11 & 0.9 & 31.7 & 7 & 237 & 20.4 & 33.1 & 18.9 & 177 & 0.29 & 0.47 & 3.0 & 3.0 & 140 \\
\hline 145 & 19.5 & 19.3 & 37.8 & 36.6 & 237 & 21.8 & 34.3 & 19.2 & 192 & 10 & 0.8 & 32.9 & 7 & 227 & 21.0 & 34.3 & 19.3 & 185 & 0.29 & 0.46 & 3.0 & 3.0 & 145 \\
\hline 150 & \begin{tabular}{|l|l}
19.8 \\
\end{tabular} & 19.6 & 38.9 & 36.6 & 227 & 22.4 & 35.5 & 19.6 & 201 & 8 & 0.8 & 34.0 & 6 & 219 & 21.7 & 35.5 & 19.7 & 194 & 0.29 & 0.45 & 3.0 & 3.0 & 150 \\
\hline
\end{tabular}




\section{Amerikaanse eik (Quercus rubra)}

Jansen, J.J., A. Oosterbaan, G.M.J. Mohren en J. den Ouden

Bron: Jansen, J.J., A. Oosterbaan, G.M.J. Mohren en J. den Ouden, 2018. Groei en productie van Amerikaanse eik in Nederland.

FEM Groei en Productie Rapport 2018 - 9, 41 blz.

Dit rapport is gratis te downloaden op:

https://doi.org/10.18174/444098

\section{Red oak}

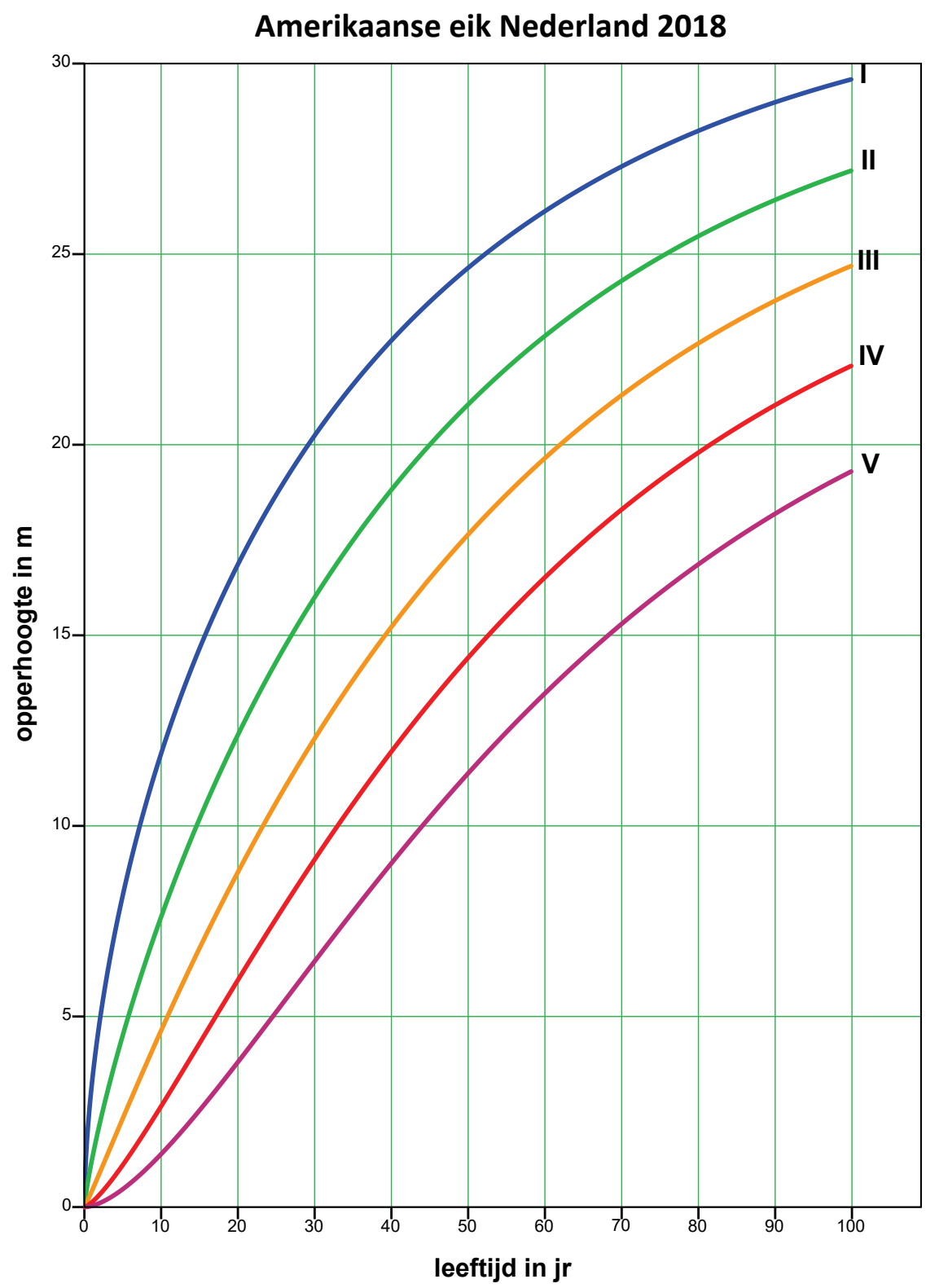




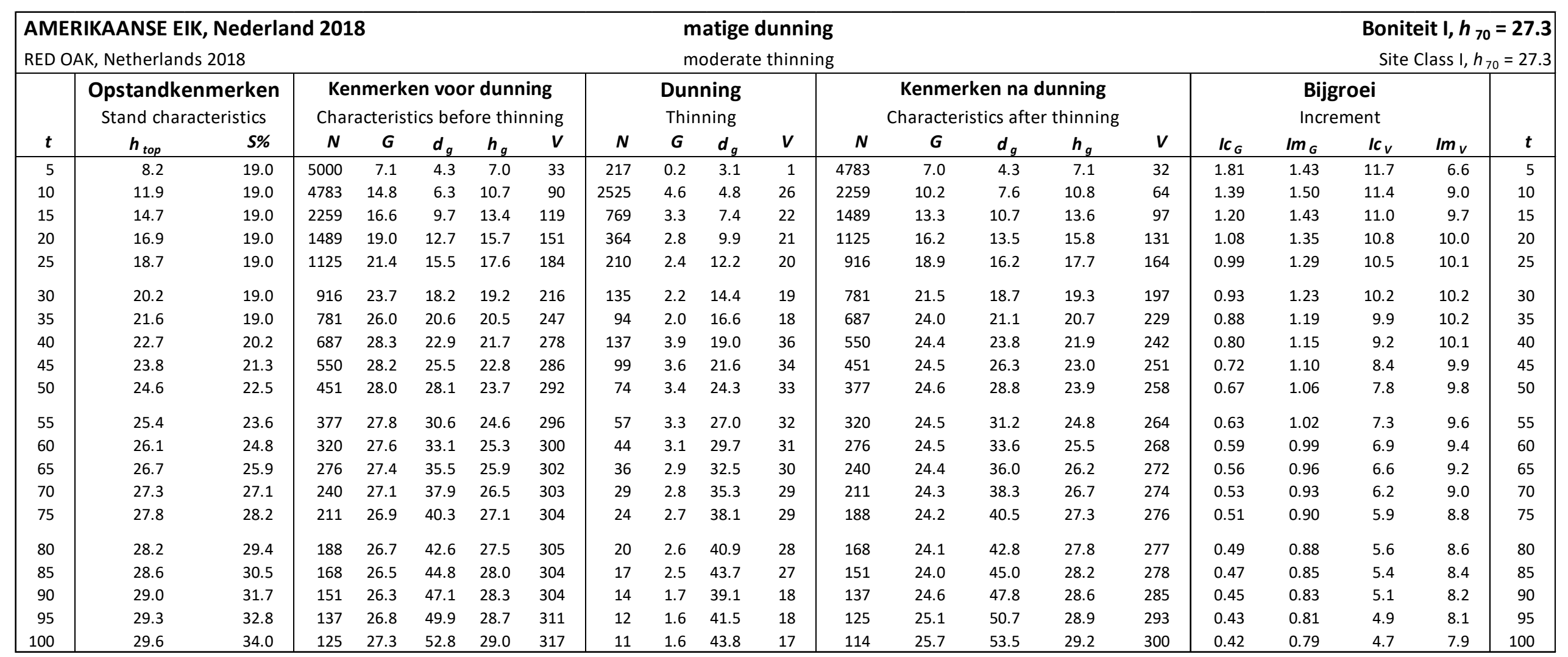




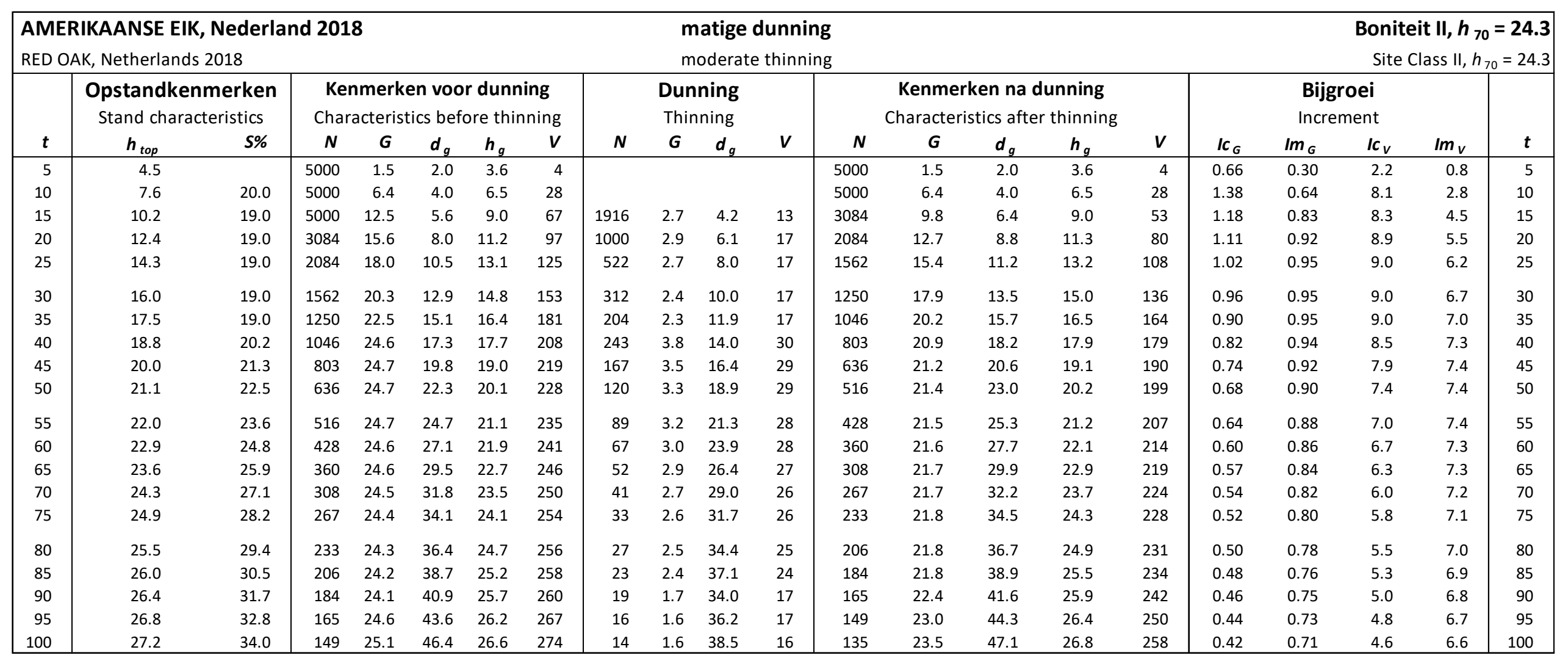




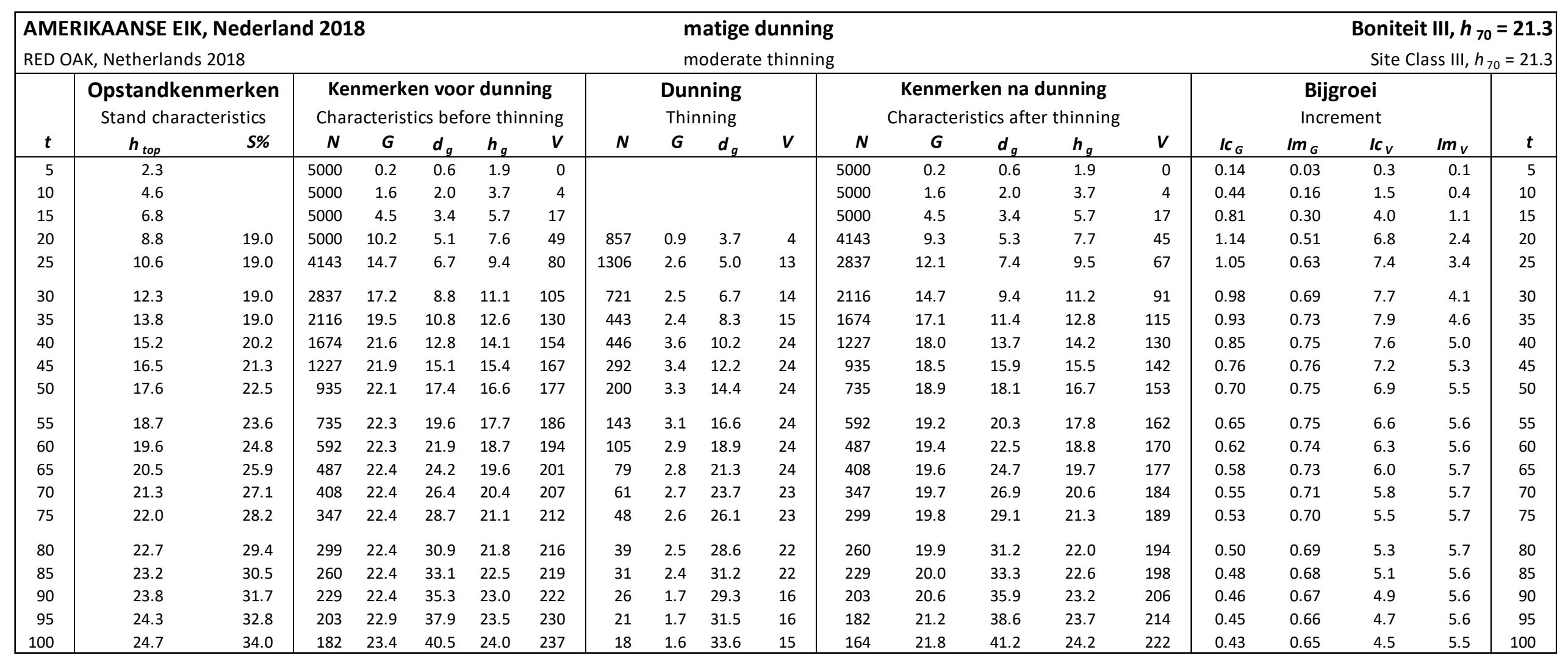




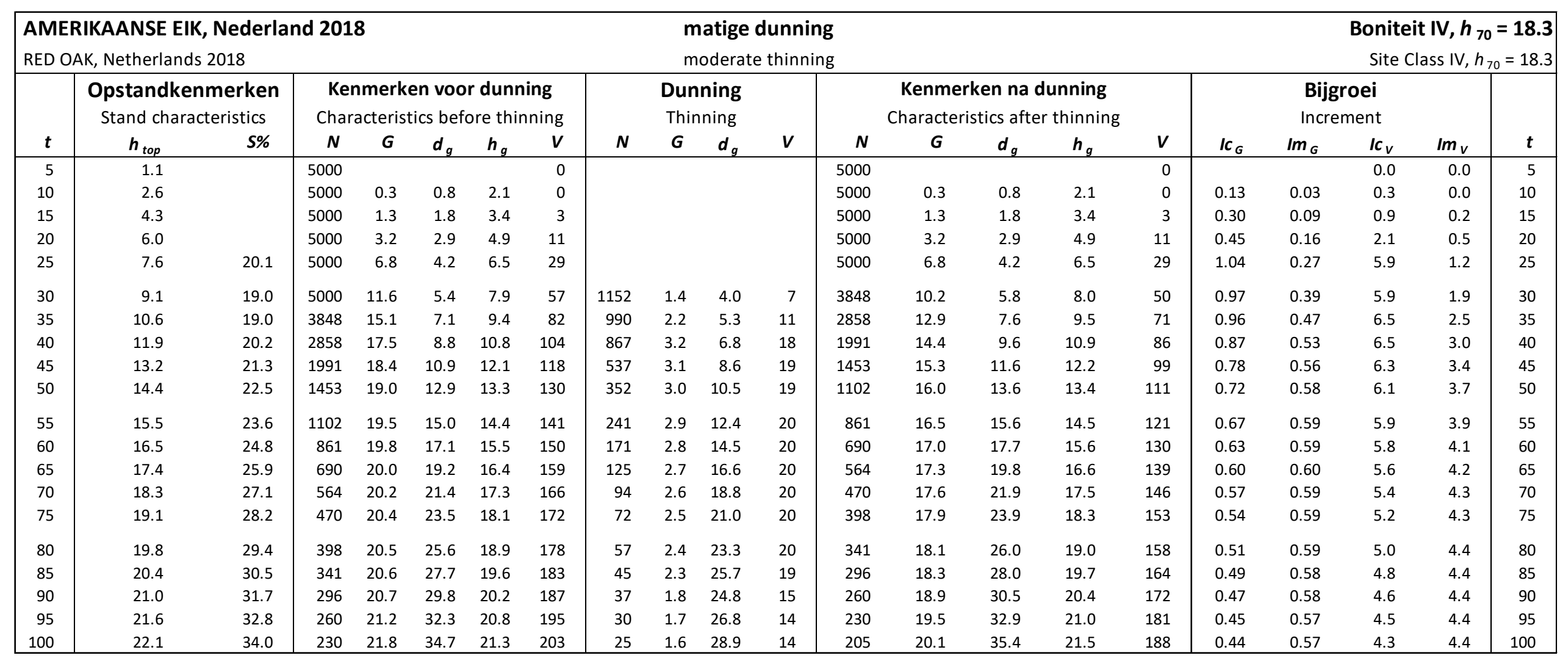




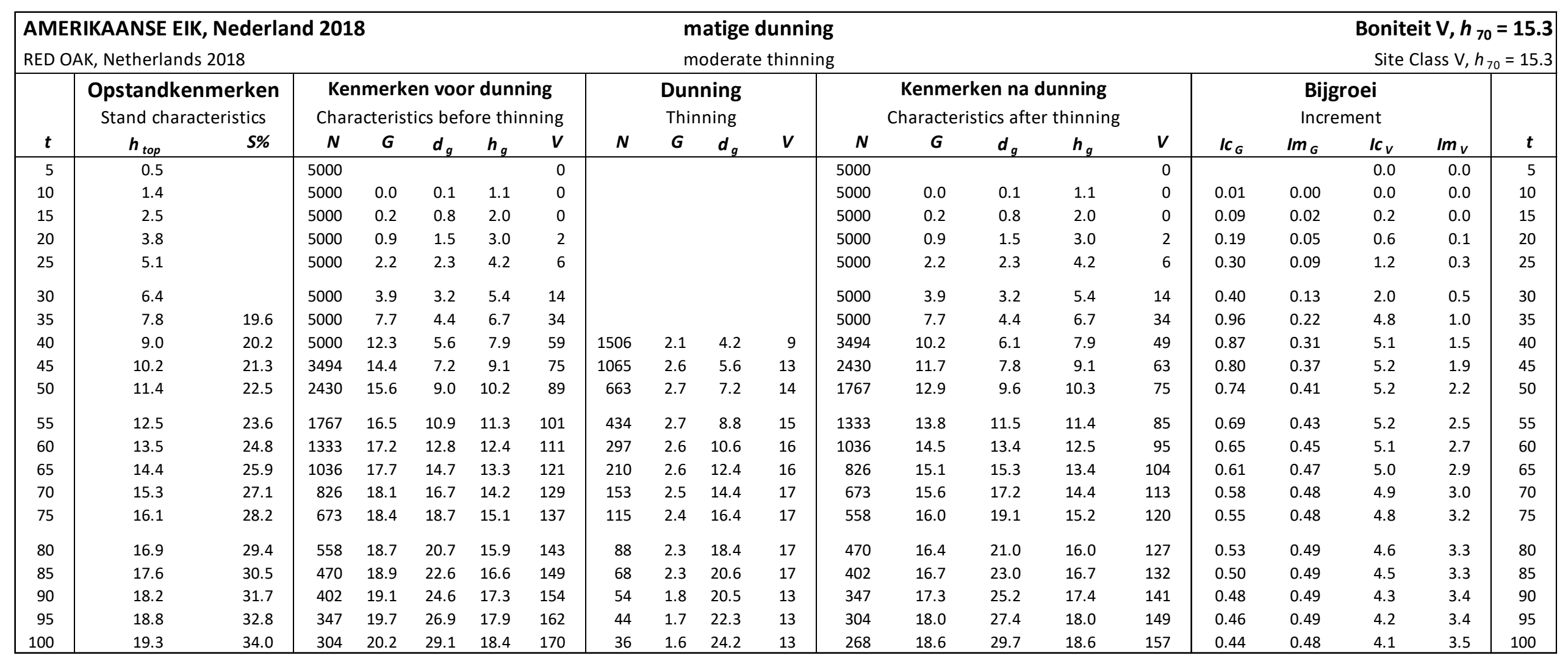




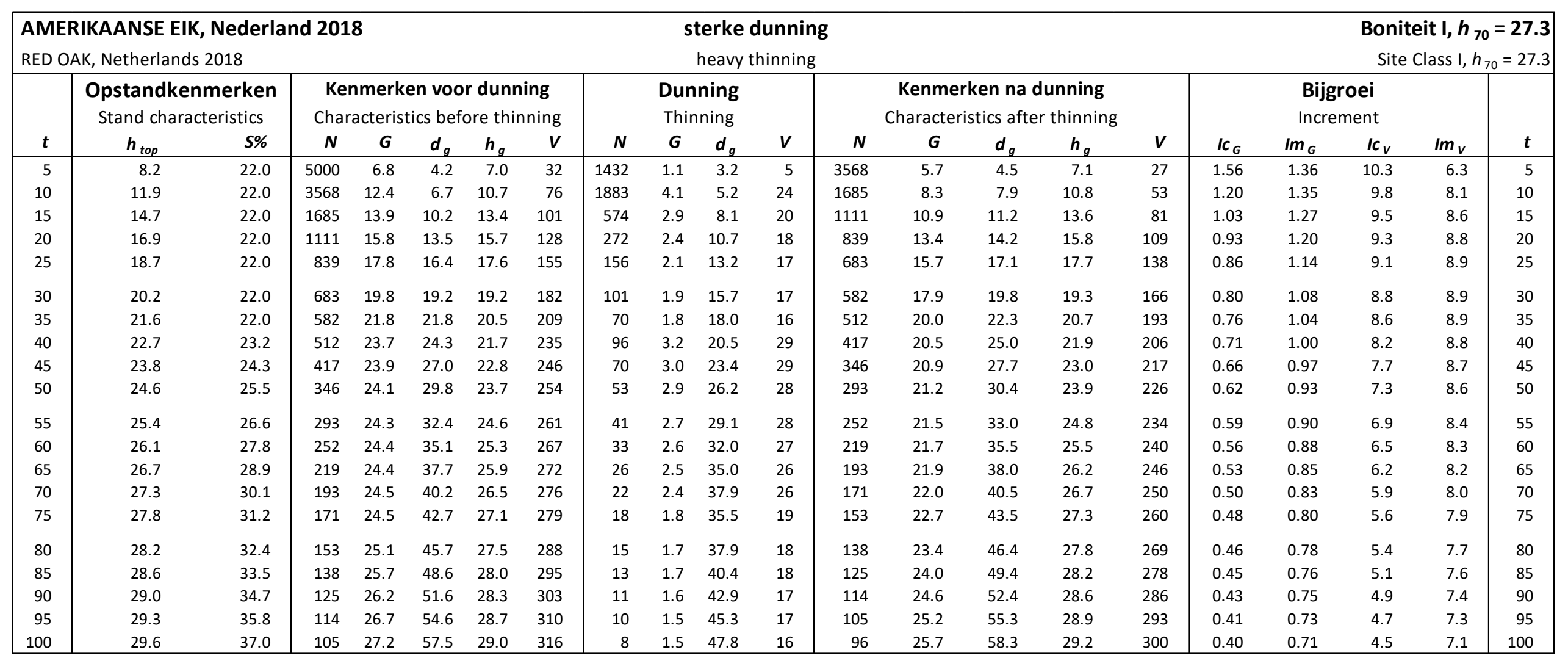




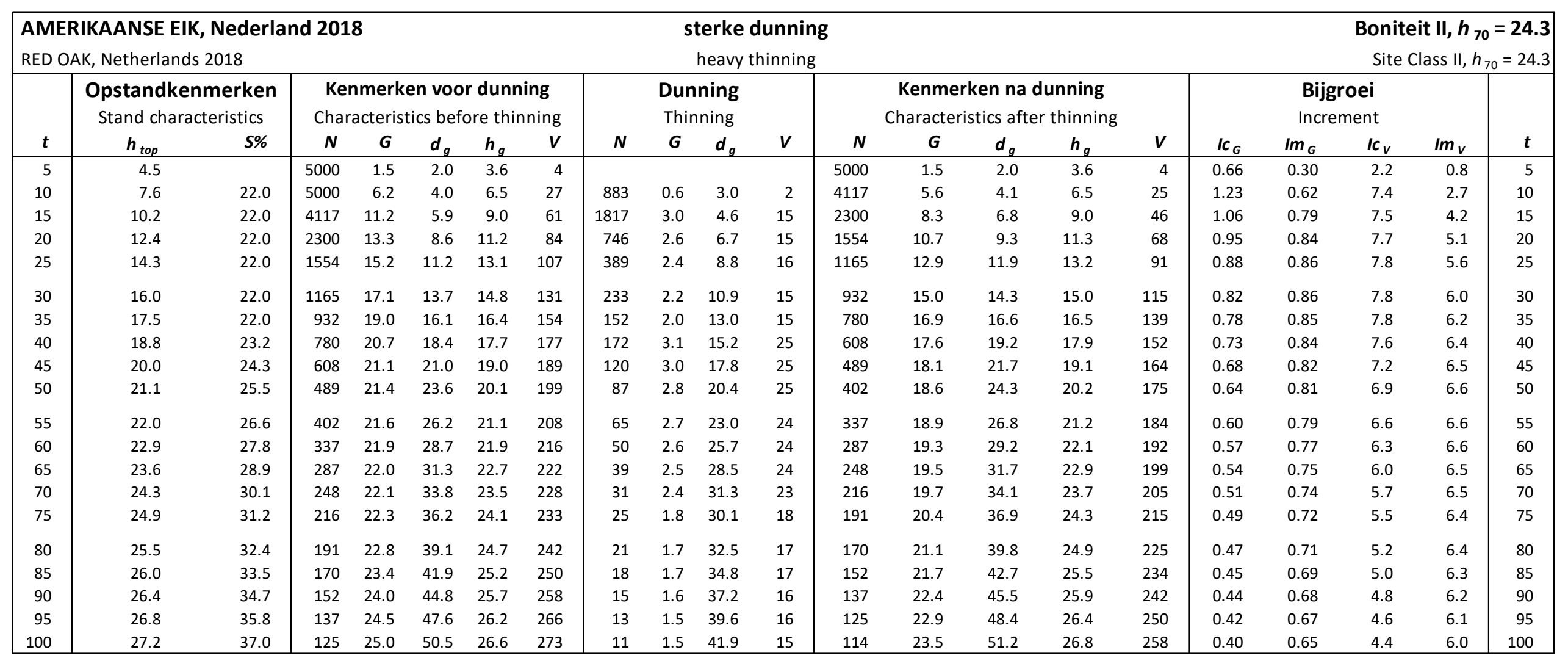




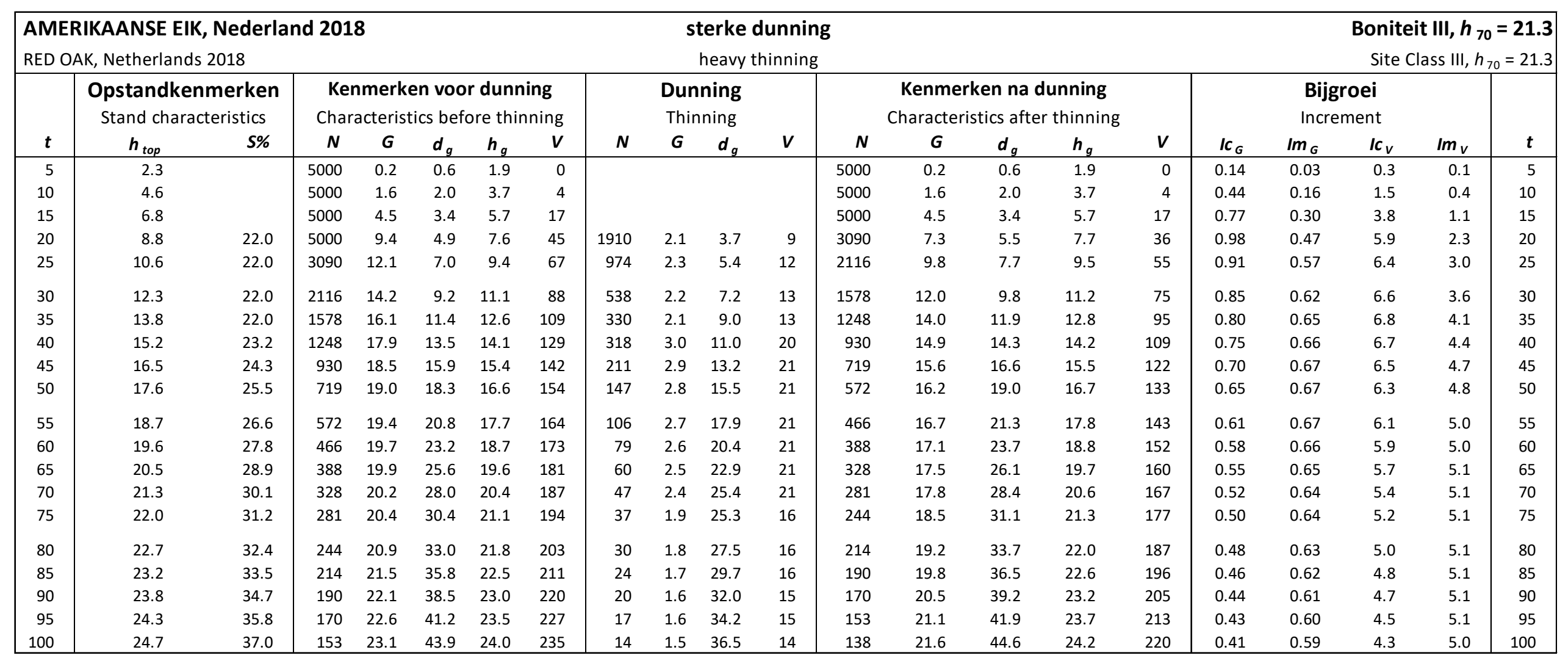




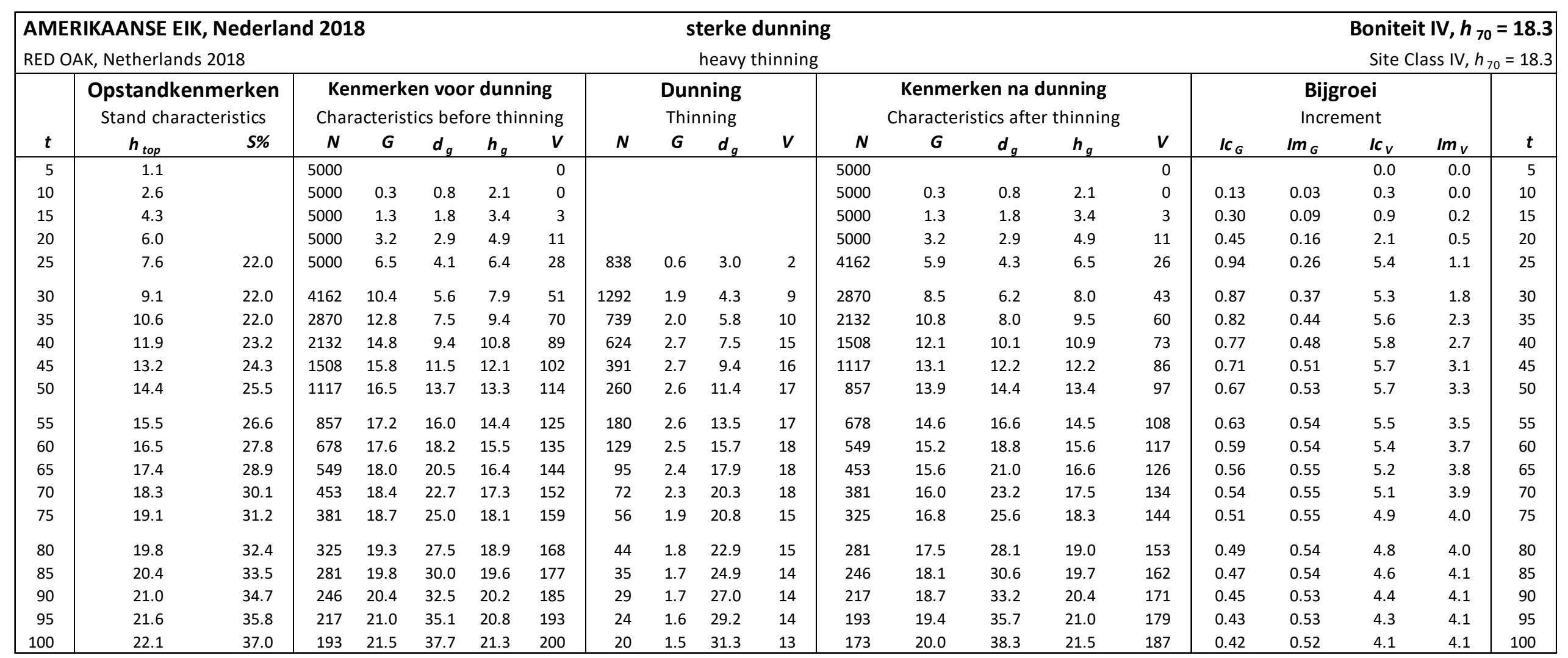




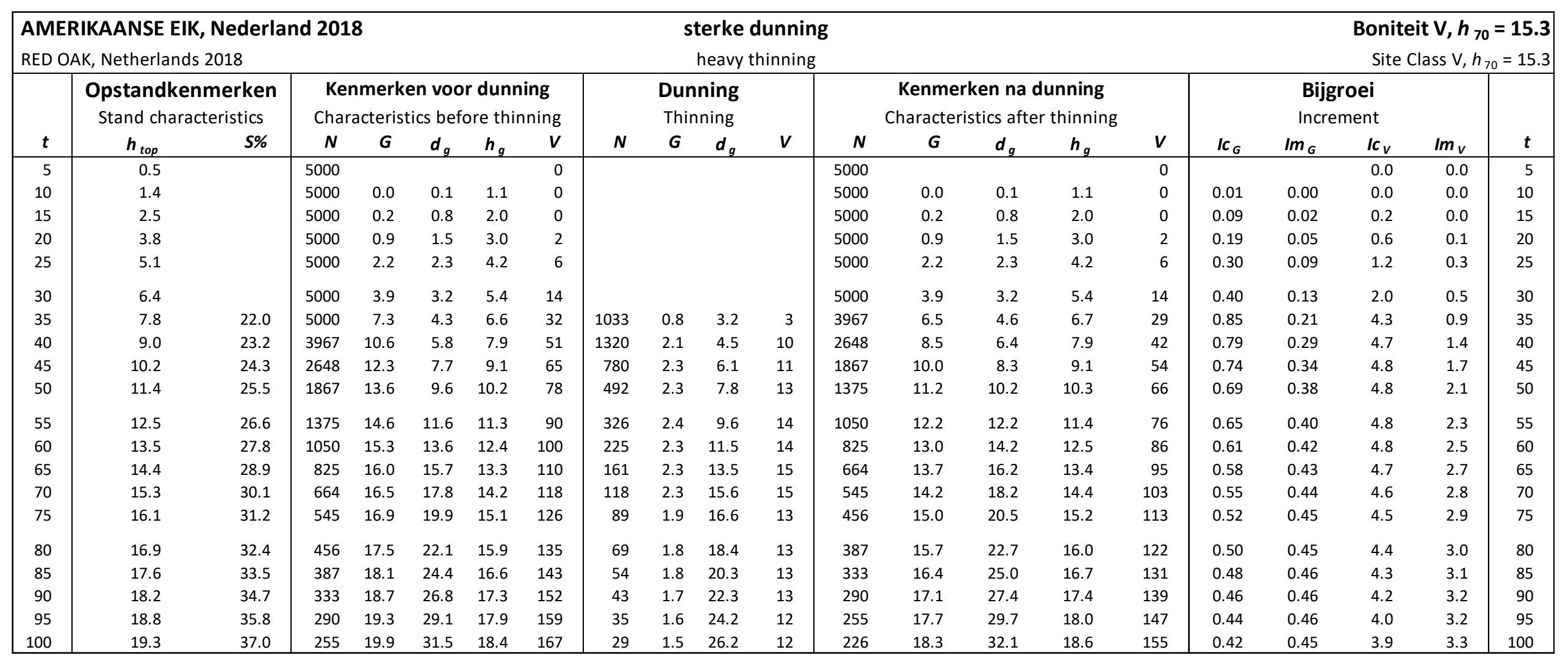




\section{Beuk (Fagus sylvatica)}

Common beech

Jansen, J.J., G.M.J. Mohren, A. Oosterbaan, L. Goudzwaard en J. den

Ouden

Bron: Jansen, J.J., G.M.J. Mohren, A. Oosterbaan, L. Goudzwaard en J. den Ouden, 2018. Groei en productie van beuk in Nederland.

FEM Groei en Productie Rapport 2018 - 5, 96 blz.

Dit rapport is gratis te downloaden op:

https://doi.org/10.18174/444094

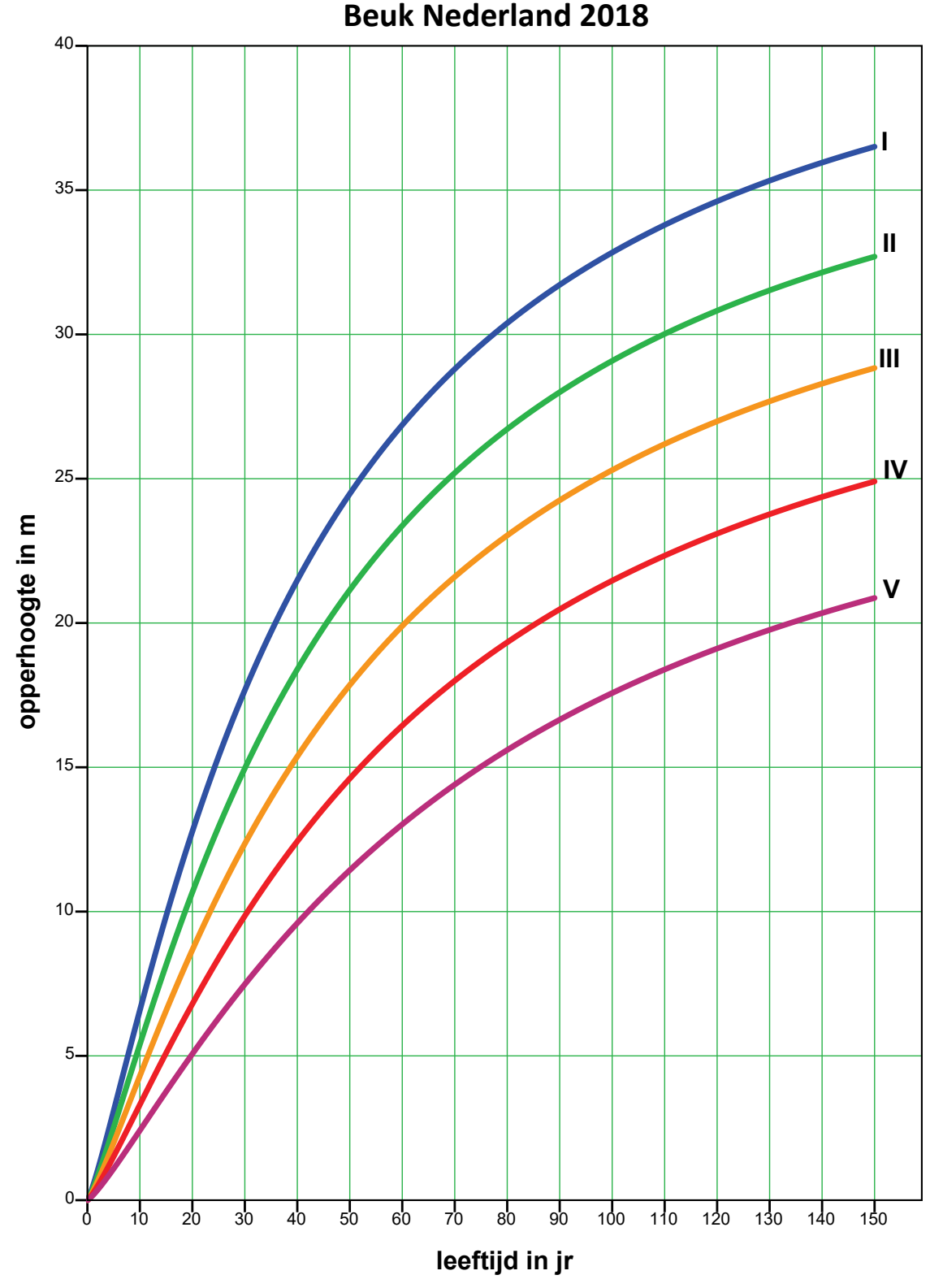




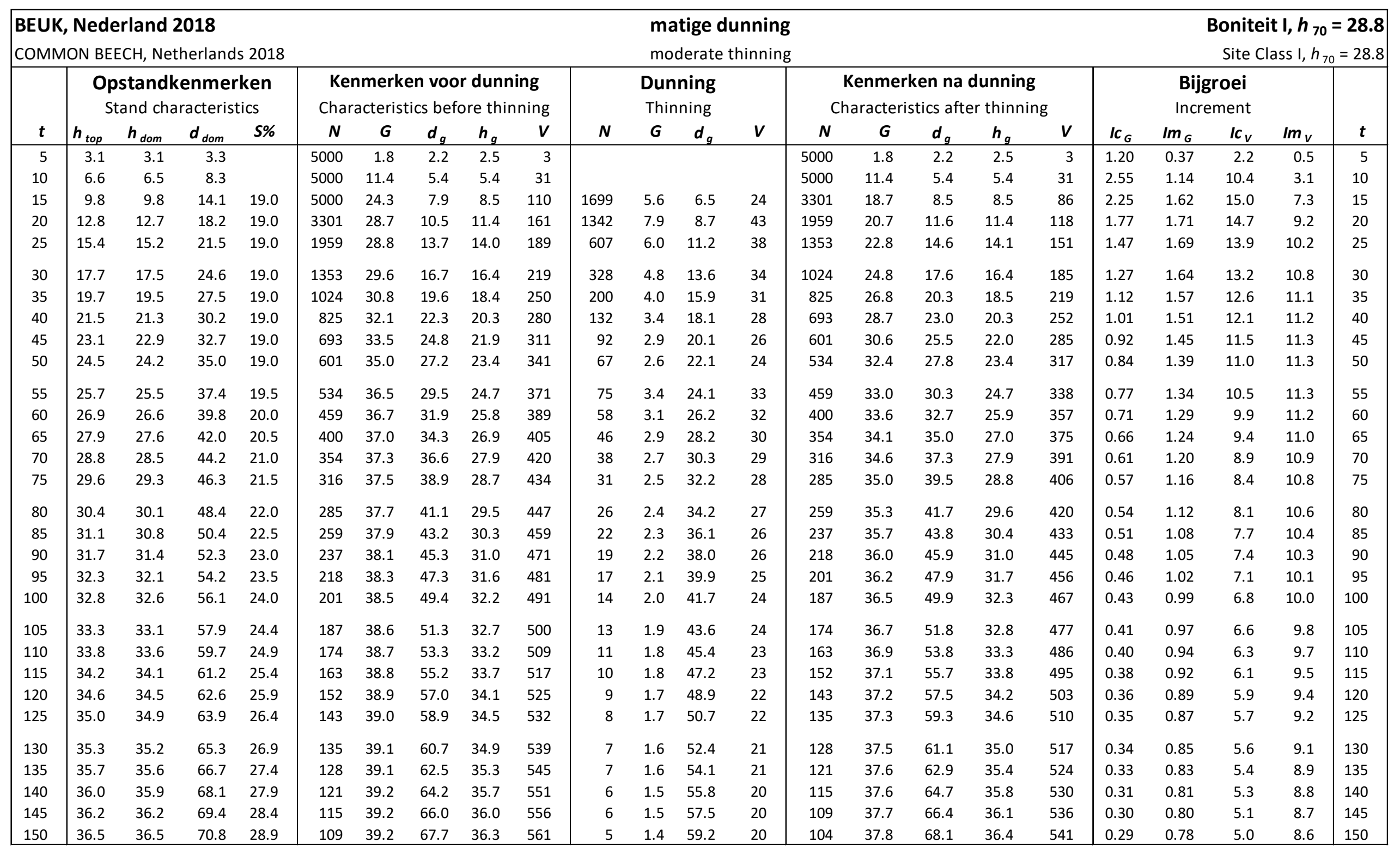




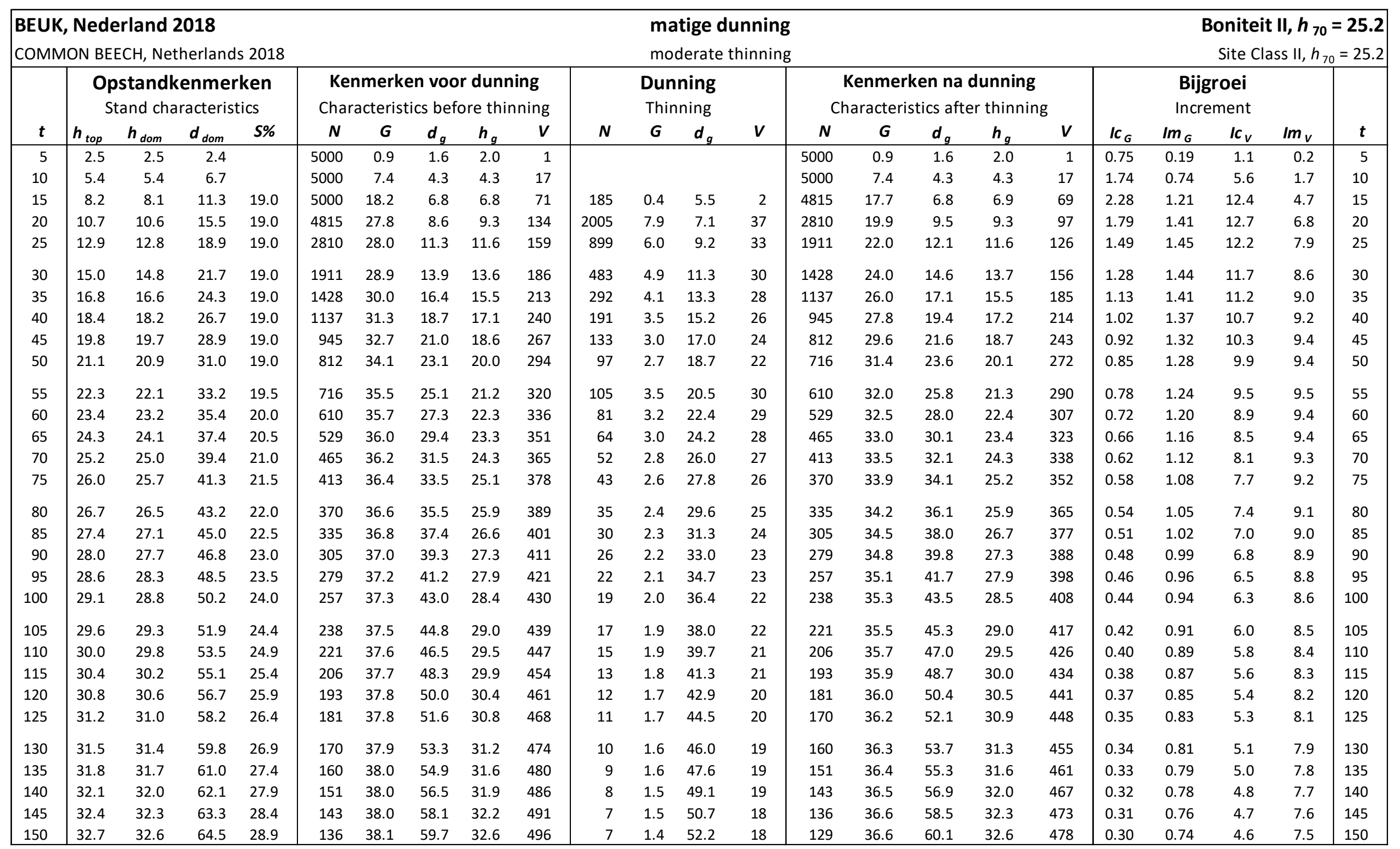




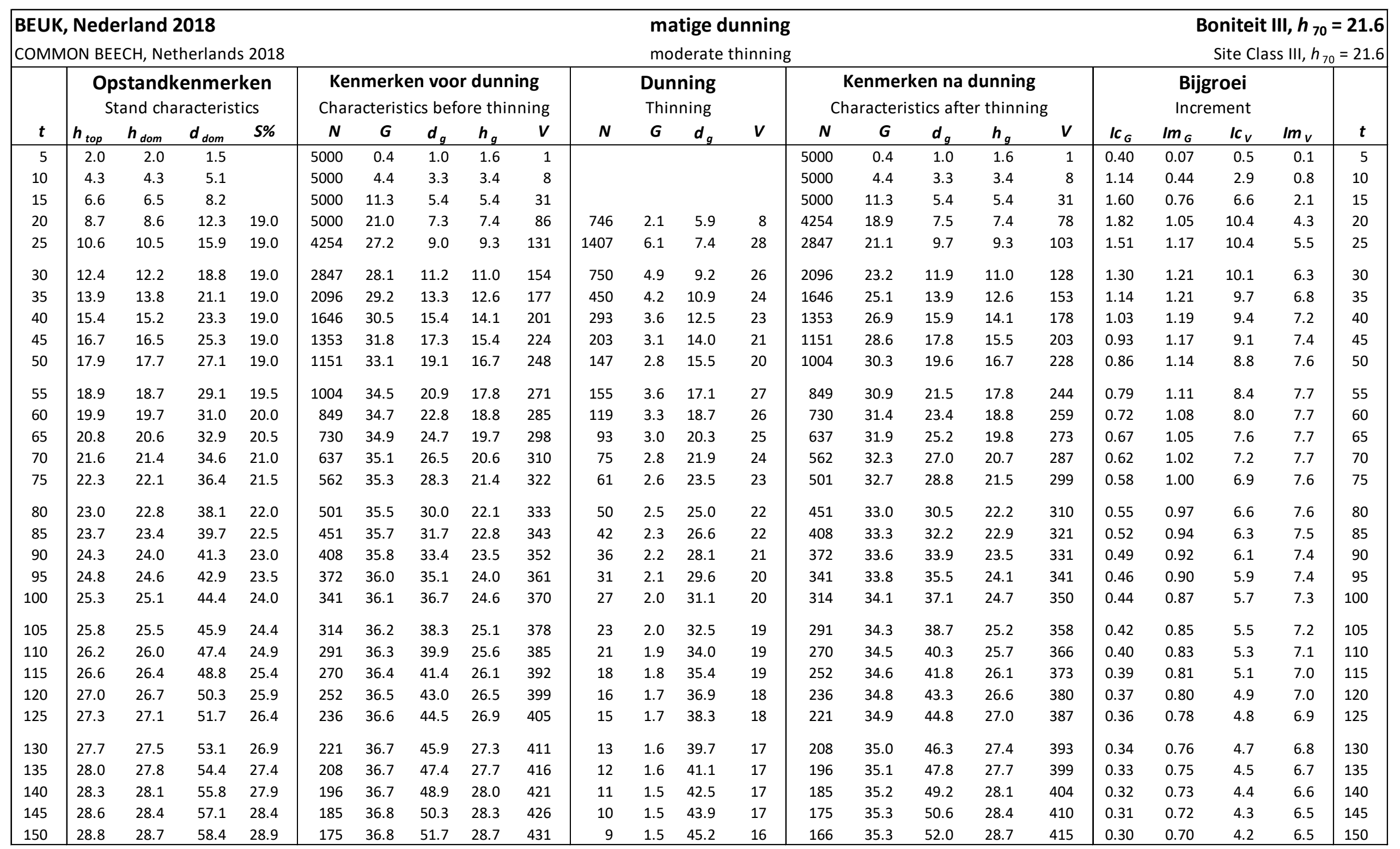




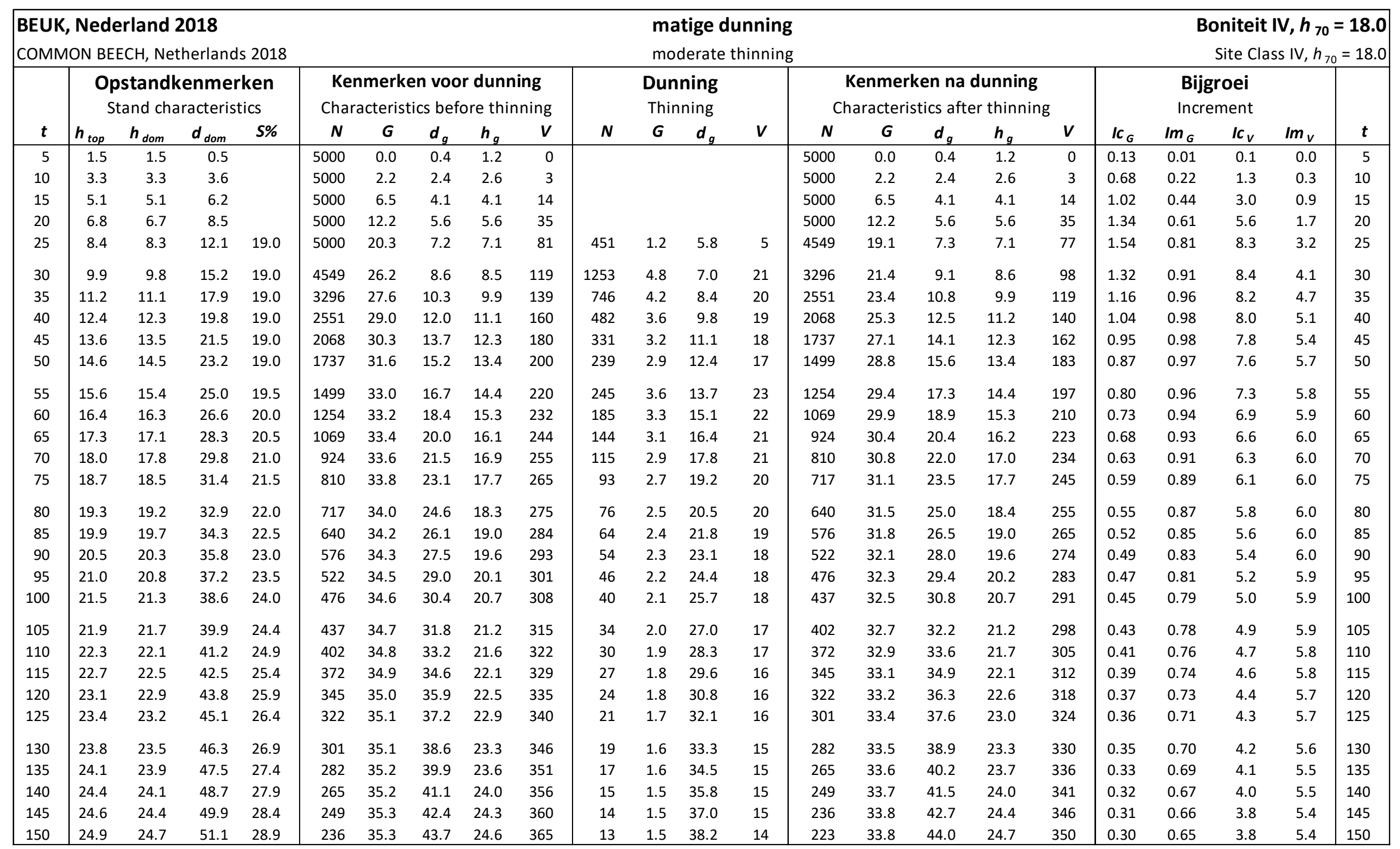




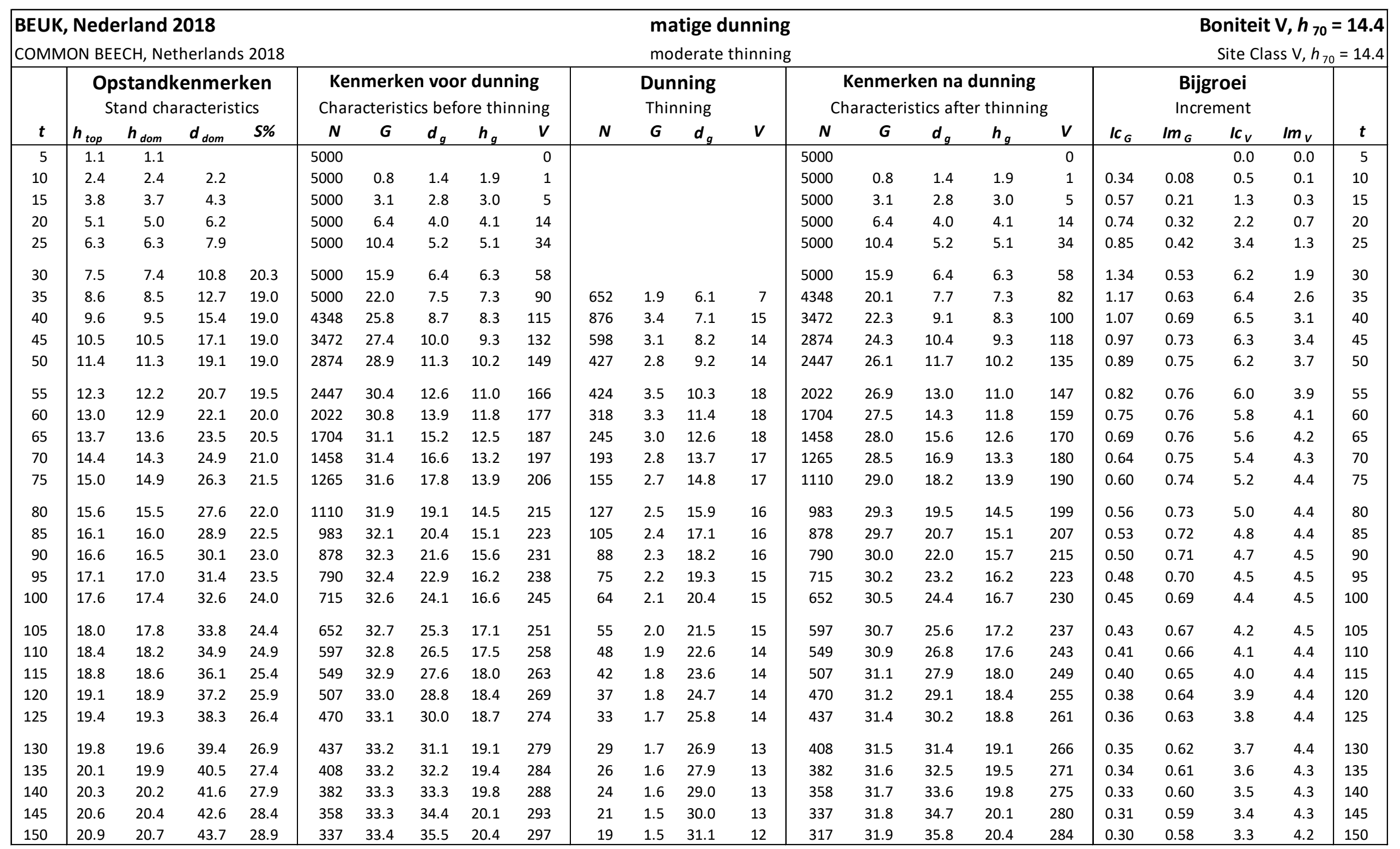




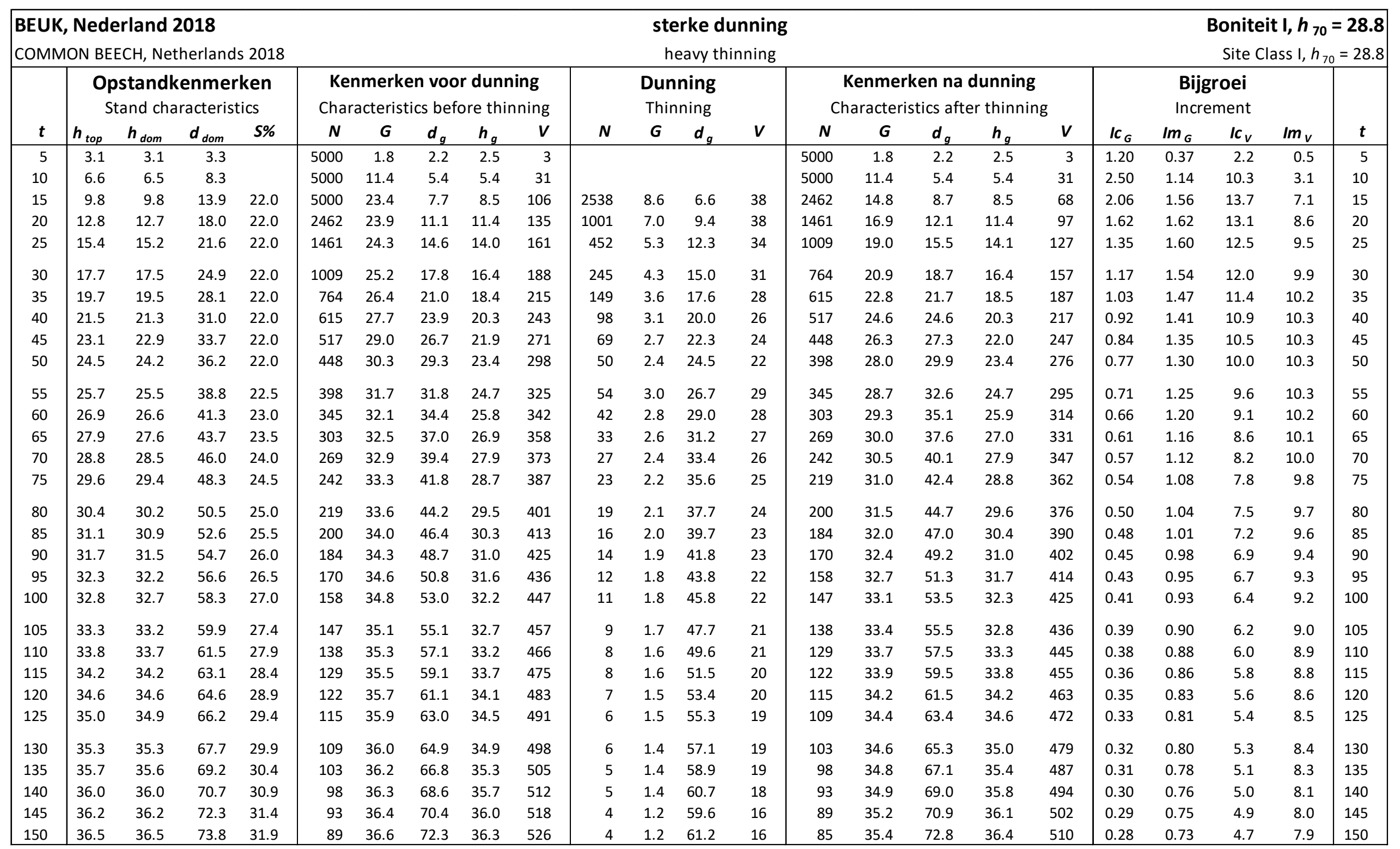




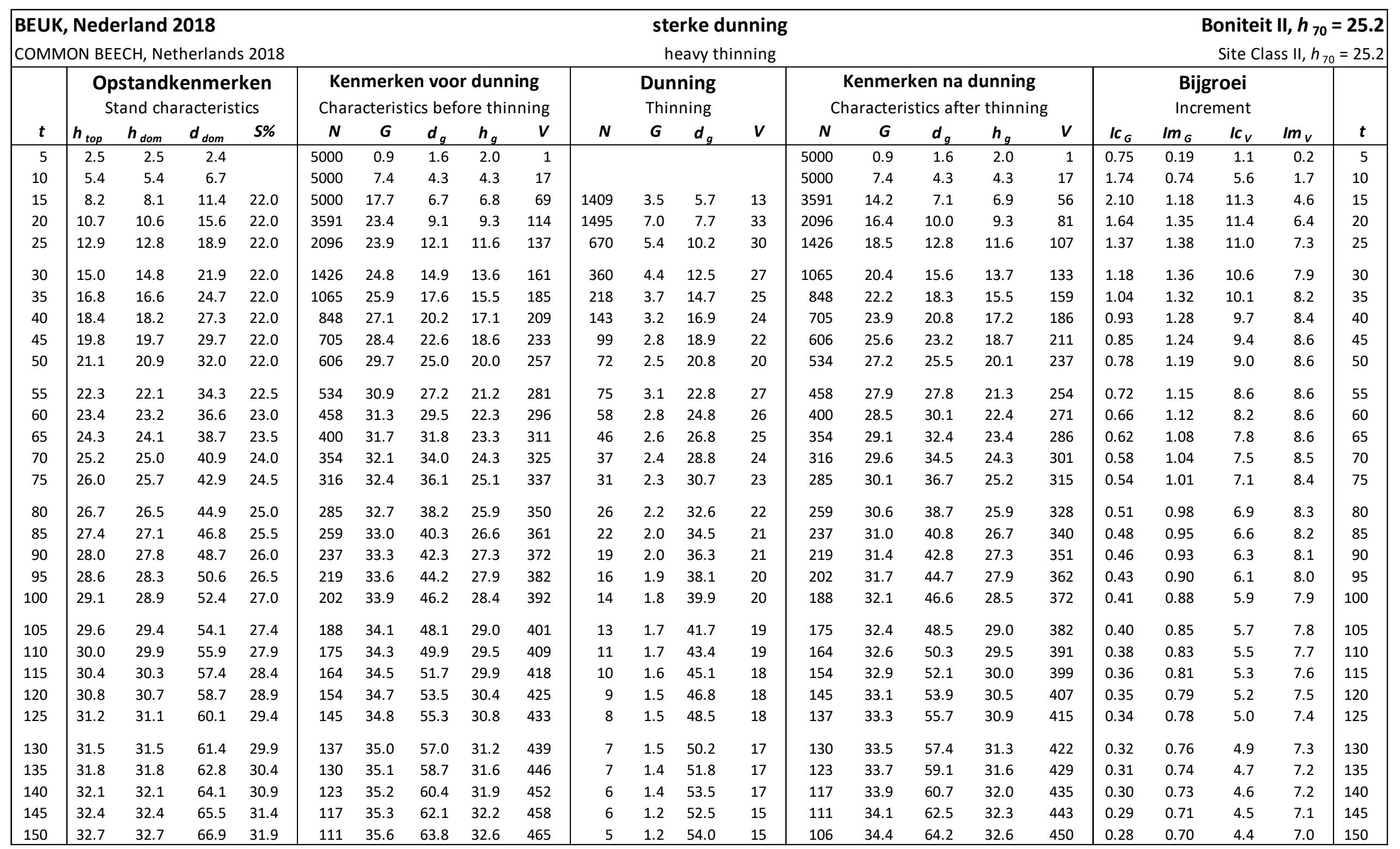




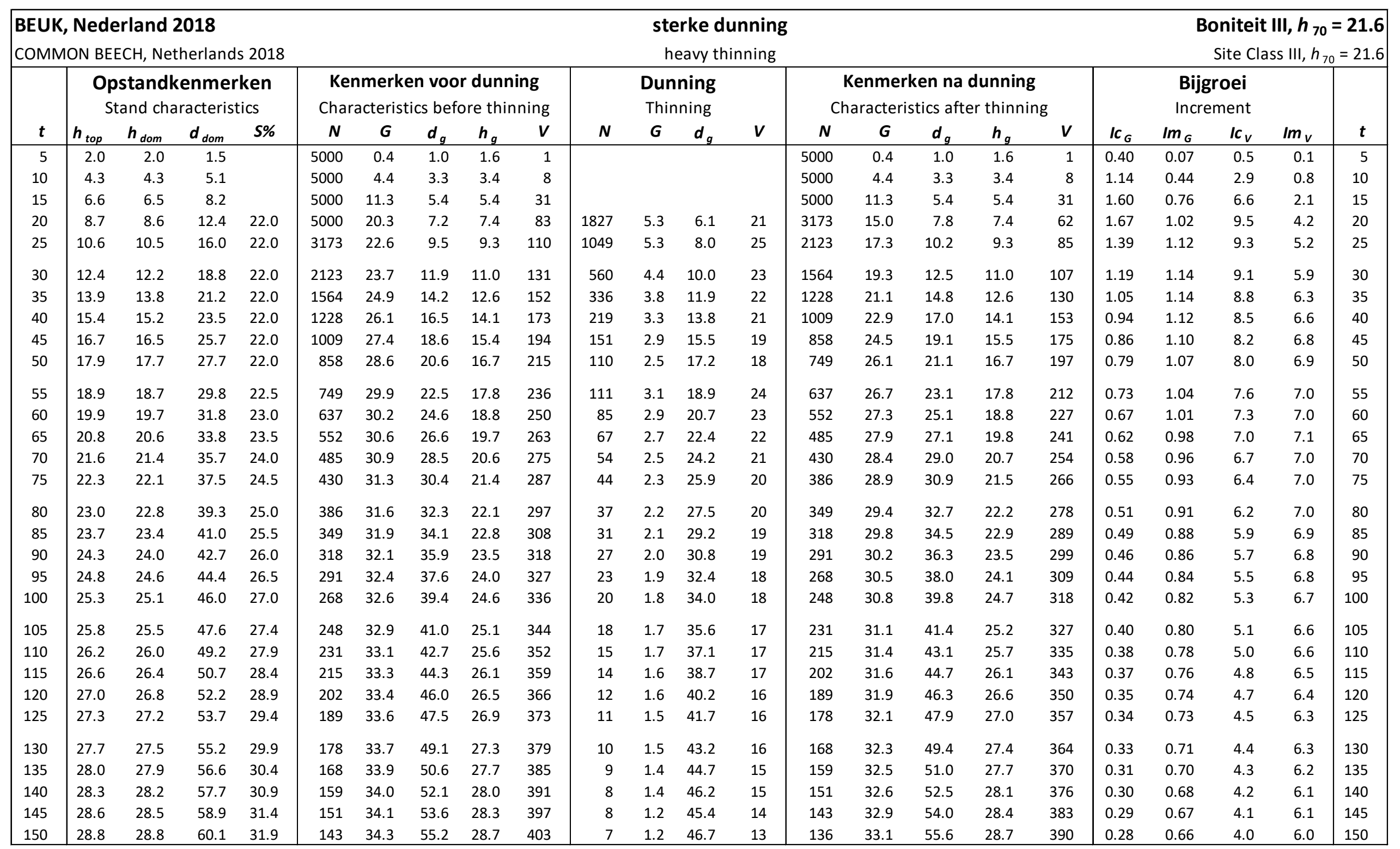




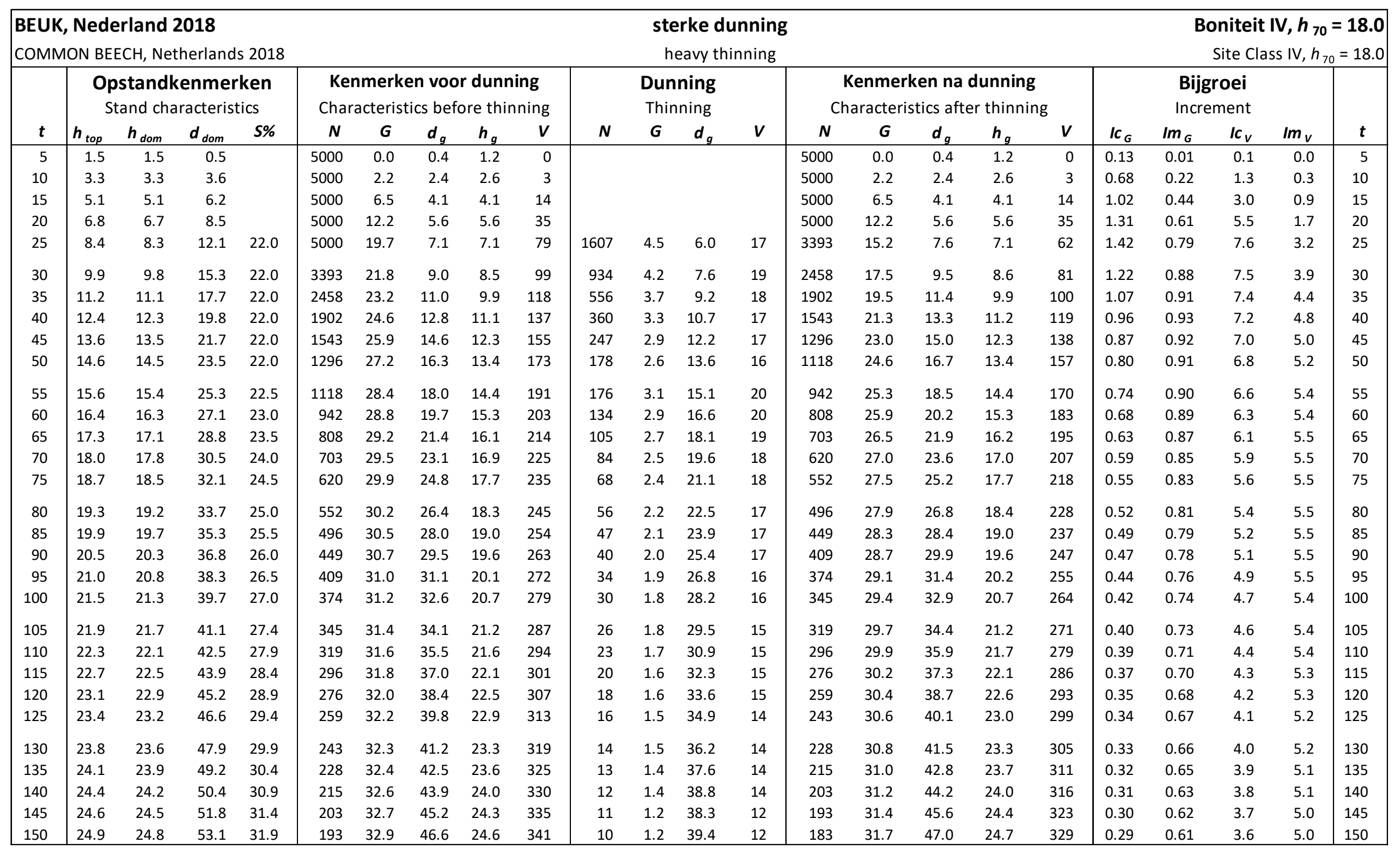




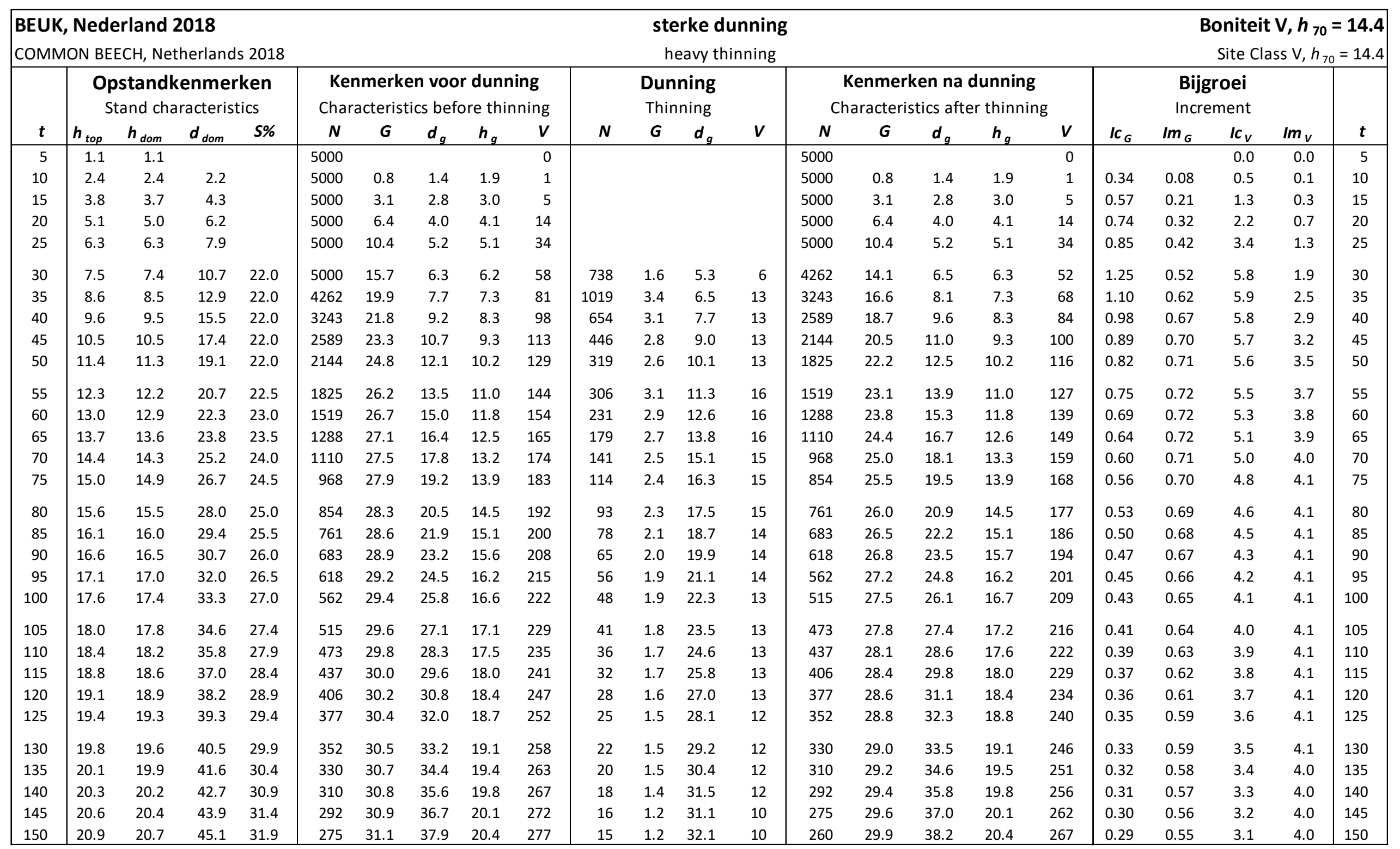




\section{Populier (Populus cultivars)}

Poplar Jansen, J.J., G.M.J. Mohren, P. Schmidt, L. Goudzwaard, A. Oosterbaan en J. den Ouden

Bron: Jansen, J.J., G.M.J. Mohren, P. Schmidt, L. Goudzwaard, A. Oosterbaan en J. den Ouden, 2018. Groei en productie van populier in Nederland. FEM Groei en Productie Rapport 2018 - 8, 127 blz.

Dit rapport is gratis te downloaden op:

https://doi.org/10.18174/444097

Het plantverband $4 \times 4$ is ontleend aan het rapport.

Bij het plantverband $5 \times 5$ en $5 \times 6$ is de in Flevoland gebruikelijke dunning en eindkap aan gehouden. Dat houdt in dat er dunning plaats vindt zodra het grondvlak boven $20 \mathrm{~m}^{2} \mathrm{ha}^{-1}$ stijgt. Voor de eindkap is de massaomloop aangehouden.

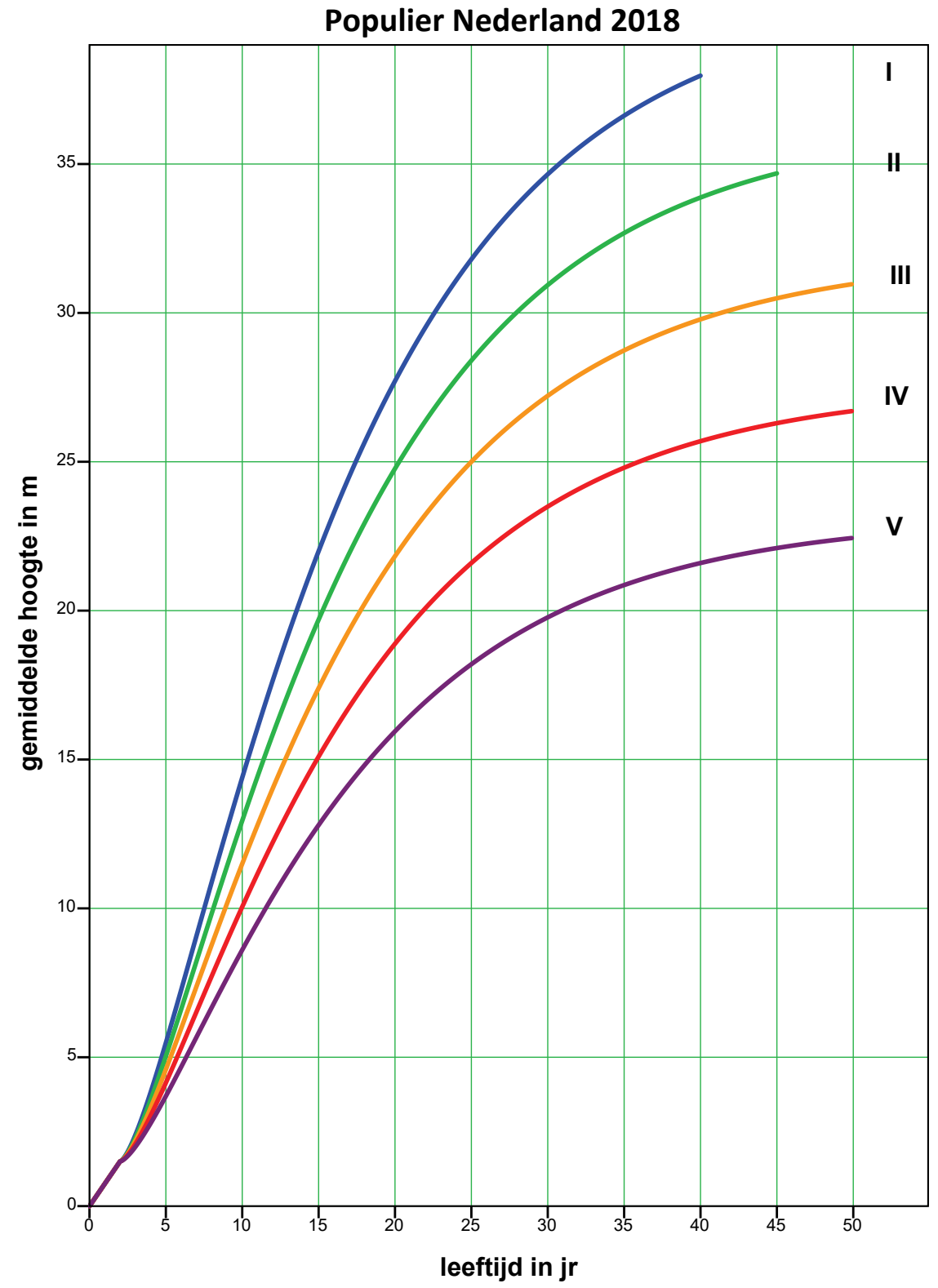




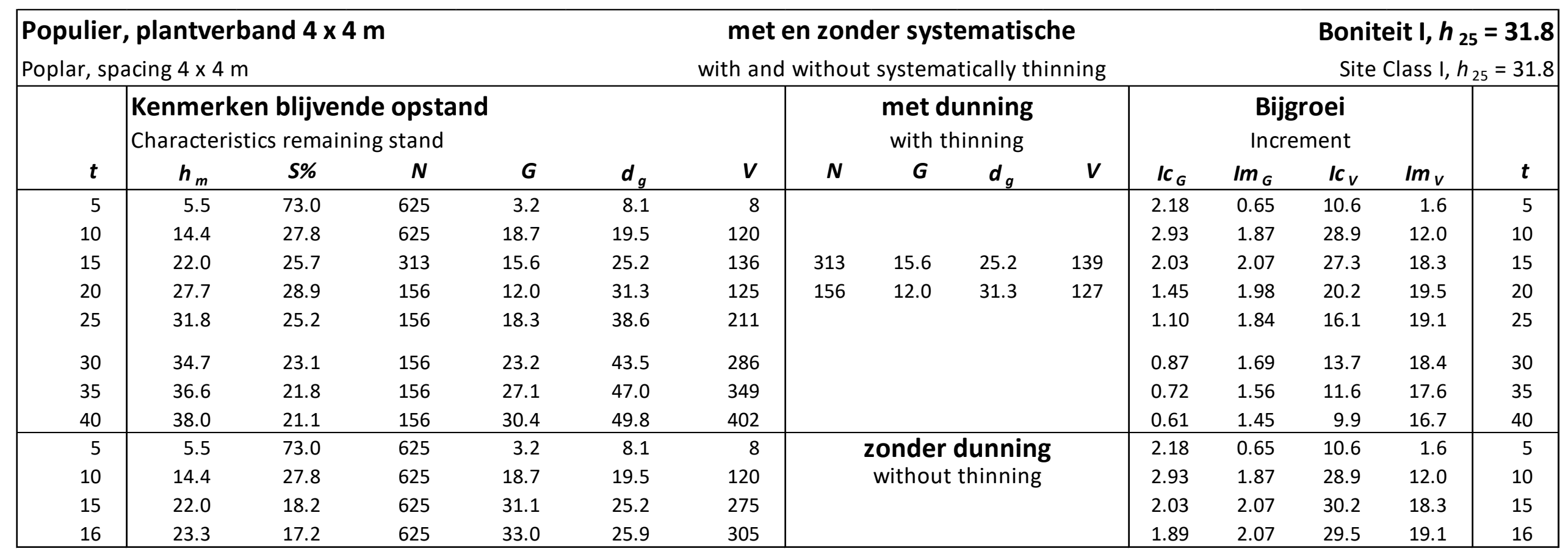




\begin{tabular}{|c|c|c|c|c|c|c|c|c|c|c|c|c|c|c|c|}
\hline \multicolumn{5}{|c|}{$\begin{array}{l}\text { Populier, plantverband } 4 \text { x } 4 \text { m } \\
\text { Poplar, spacing } 4 \times 4 \mathrm{~m}\end{array}$} & \multicolumn{7}{|c|}{$\begin{array}{l}\text { met en zonder systematische } \\
\text { with and without systematically thinning }\end{array}$} & \multicolumn{4}{|c|}{$\begin{array}{r}\text { Boniteit II, } \boldsymbol{h}_{\mathbf{2 5}}=\mathbf{2 8 . 4} \\
\text { Site Class II, } h_{25}=28.4\end{array}$} \\
\hline \multirow[b]{2}{*}{$t$} & \multicolumn{6}{|c|}{$\begin{array}{l}\text { Kenmerken blijvende opstand } \\
\text { Characteristics remaining stand }\end{array}$} & \multicolumn{4}{|c|}{$\begin{array}{l}\text { met dunning } \\
\text { with thinning }\end{array}$} & \multicolumn{4}{|c|}{$\begin{array}{c}\text { Bijgroei } \\
\text { Increment }\end{array}$} & \multirow[b]{2}{*}{$t$} \\
\hline & $\boldsymbol{h}_{m}$ & $S \%$ & $N$ & $\boldsymbol{G}$ & $d_{g}$ & $V$ & $N$ & $\boldsymbol{G}$ & $d_{g}$ & $v$ & $I c_{G}$ & $I m_{G}$ & $I c_{V}$ & $I m_{V}$ & \\
\hline 5 & 5.0 & 79.5 & 625 & 2.5 & 7.2 & 6 & \multirow{4}{*}{313} & \multirow{4}{*}{13.6} & \multirow{4}{*}{23.5} & \multirow{4}{*}{111} & 1.83 & 0.51 & 8.3 & 1.2 & 5 \\
\hline 10 & 12.9 & 30.9 & 625 & 15.9 & 18.0 & 94 & & & & & 2.58 & 1.59 & 23.2 & 9.4 & 10 \\
\hline 15 & 19.7 & 28.7 & 313 & 13.6 & 23.5 & 109 & & & & & 1.87 & 1.81 & 22.6 & 14.7 & 15 \\
\hline 20 & 24.8 & 22.8 & 313 & 21.4 & 29.5 & 206 & & & & & 1.34 & 1.75 & 18.2 & 15.9 & 20 \\
\hline 25 & 28.4 & 28.2 & 156 & 13.6 & 33.3 & 144 & \multirow[t]{5}{*}{156} & \multirow[t]{5}{*}{13.6} & \multirow[t]{5}{*}{33.3} & \multirow[t]{5}{*}{146} & 1.02 & 1.63 & 14.4 & 16.1 & 25 \\
\hline 30 & 30.9 & 25.9 & 156 & 18.1 & 38.4 & 205 & & & & & 0.81 & 1.51 & 11.3 & 15.4 & 30 \\
\hline 35 & 32.7 & 24.5 & 156 & 21.7 & 42.1 & 257 & & & & & 0.67 & 1.40 & 9.6 & 14.7 & 35 \\
\hline 40 & 33.9 & 23.6 & 156 & 24.8 & 45.0 & 301 & & & & & 0.57 & 1.30 & 8.2 & 14.0 & 40 \\
\hline 45 & 34.7 & 23.1 & 156 & 27.4 & 47.3 & 339 & & & & & 0.49 & 1.21 & 7.2 & 13.3 & 45 \\
\hline 5 & 5.0 & 79.5 & 625 & 2.5 & 7.2 & 6 & \multirow{4}{*}{\multicolumn{4}{|c|}{$\begin{array}{l}\text { zonder dunning } \\
\text { without thinning }\end{array}$}} & 1.83 & 0.51 & 8.3 & 1.2 & 5 \\
\hline 10 & 12.9 & 30.9 & 625 & 15.9 & 18.0 & 94 & & & & & 2.58 & 1.59 & 23.2 & 9.4 & 10 \\
\hline 15 & 19.7 & 20.3 & 625 & 27.1 & 23.5 & 221 & & & & & 1.87 & 1.81 & 24.9 & 14.7 & 15 \\
\hline 18 & 22.9 & 17.4 & 625 & 32.1 & 25.6 & 293 & & & & & 1.52 & 1.79 & 23.1 & 16.3 & 18 \\
\hline
\end{tabular}




\begin{tabular}{|c|c|c|c|c|c|c|c|c|c|c|c|c|c|c|c|}
\hline \multicolumn{5}{|c|}{$\begin{array}{l}\text { Populier, plantverband } 4 \text { × } 4 \text { m } \\
\text { Poplar, spacing } 4 \times 4 \mathrm{~m}\end{array}$} & \multicolumn{6}{|c|}{$\begin{array}{l}\text { met en zonder systematische } \\
\text { with and without systematically thinning }\end{array}$} & \multicolumn{5}{|c|}{$\begin{array}{r}\text { Boniteit III, } \boldsymbol{h}_{\mathbf{2 5}}=\mathbf{2 5 . 0} \\
\text { Site Class III, } h_{25}=25.0 \\
\end{array}$} \\
\hline \multirow[b]{2}{*}{$t$} & \multicolumn{6}{|c|}{$\begin{array}{l}\text { Kenmerken blijvende opstand } \\
\text { Characteristics remaining stand }\end{array}$} & \multicolumn{4}{|c|}{$\begin{array}{l}\text { met dunning } \\
\text { with thinning }\end{array}$} & \multicolumn{4}{|c|}{$\begin{array}{l}\text { Bijgroei } \\
\text { Increment }\end{array}$} & \multirow[b]{2}{*}{$t$} \\
\hline & $\boldsymbol{h}_{m}$ & $S \%$ & $N$ & $\boldsymbol{G}$ & $d_{g}$ & $V$ & $N$ & $\boldsymbol{G}$ & $d_{g}$ & $V$ & $I c_{G}$ & $I m_{G}$ & $I c_{v}$ & $I m_{v}$ & \\
\hline 5 & 4.6 & 87.2 & 625 & 1.9 & 6.2 & 4 & \multirow{5}{*}{313} & \multirow{5}{*}{15.1} & \multirow{5}{*}{24.8} & \multirow{5}{*}{134} & 1.51 & 0.38 & 6.3 & 0.8 & 5 \\
\hline 10 & 11.5 & 34.8 & 625 & 13.2 & 16.4 & 71 & & & & & 2.19 & 1.32 & 17.8 & 7.1 & 10 \\
\hline 15 & 17.4 & 23.0 & 625 & 23.1 & 21.7 & 171 & & & & & 1.69 & 1.54 & 20.0 & 11.4 & 15 \\
\hline 20 & 21.8 & 25.9 & 313 & 15.1 & 24.8 & 132 & & & & & 1.22 & 1.51 & 15.9 & 13.3 & 20 \\
\hline 25 & 25.0 & 22.6 & 313 & 20.4 & 28.8 & 198 & & & & & 0.93 & 1.42 & 12.2 & 13.3 & 25 \\
\hline 30 & 27.2 & 20.8 & 313 & 24.6 & 31.6 & 253 & \multirow{5}{*}{156} & \multirow{5}{*}{14.0} & \multirow{5}{*}{33.7} & \multirow{5}{*}{151} & 0.74 & 1.32 & 10.3 & 12.9 & 30 \\
\hline 35 & 28.7 & 27.8 & 156 & 14.0 & 33.7 & 149 & & & & & 0.61 & 1.23 & 8.2 & 12.4 & 35 \\
\hline 40 & 29.8 & 26.9 & 156 & 16.8 & 37.0 & 184 & & & & & 0.52 & 1.15 & 6.6 & 11.7 & 40 \\
\hline 45 & 30.5 & 26.2 & 156 & 19.2 & 39.6 & 215 & & & & & 0.46 & 1.07 & 5.8 & 11.1 & 45 \\
\hline 50 & 31.0 & 25.8 & 156 & 21.4 & 41.7 & 243 & & & & & 0.41 & 1.01 & 5.2 & 10.6 & 50 \\
\hline 5 & 4.6 & 87.2 & 625 & 1.9 & 6.2 & 4 & \multirow{5}{*}{\multicolumn{4}{|c|}{$\begin{array}{l}\text { zonder dunning } \\
\text { without thinning }\end{array}$}} & 1.51 & 0.38 & 6.3 & 0.8 & 5 \\
\hline 10 & 11.5 & 34.8 & 625 & 13.2 & 16.4 & 71 & & & & & 2.19 & 1.32 & 17.8 & 7.1 & 10 \\
\hline 15 & 17.4 & 23.0 & 625 & 23.1 & 21.7 & 171 & & & & & 1.69 & 1.54 & 20.0 & 11.4 & 15 \\
\hline 20 & 21.8 & 18.3 & 625 & 30.3 & 24.8 & 266 & & & & & 1.22 & 1.51 & 17.5 & 13.3 & 20 \\
\hline 22 & 23.2 & 17.2 & 625 & 32.6 & 25.8 & 300 & & & & & 1.09 & 1.48 & 16.3 & 13.6 & 22 \\
\hline
\end{tabular}




\begin{tabular}{|c|c|c|c|c|c|c|c|c|c|c|c|c|c|c|c|}
\hline \multirow{2}{*}{\multicolumn{5}{|c|}{$\begin{array}{l}\text { Populier, plantverband } 4 \text { x } 4 \text { m } \\
\text { Poplar, spacing } 4 \times 4 \mathrm{~m}\end{array}$}} & \multirow{2}{*}{\multicolumn{7}{|c|}{$\begin{array}{l}\text { met en zonder systematische } \\
\text { with and without systematically thinning }\end{array}$}} & \multirow{2}{*}{\multicolumn{4}{|c|}{$\begin{array}{r}\text { Boniteit IV, } \boldsymbol{h}_{\mathbf{2 5}}=\mathbf{2 1 . 6} \\
\text { Site Class IV, } h_{25}=21.6\end{array}$}} \\
\hline & & & & & & & & & & & & & & & \\
\hline \multirow[b]{2}{*}{$t$} & \multicolumn{6}{|c|}{$\begin{array}{l}\text { Kenmerken blijvende opstand } \\
\text { Characteristics remaining stand }\end{array}$} & \multicolumn{4}{|c|}{$\begin{array}{l}\text { met dunning } \\
\text { with thinning }\end{array}$} & \multicolumn{4}{|c|}{$\begin{array}{l}\text { Bijgroei } \\
\text { Increment }\end{array}$} & \multirow[b]{2}{*}{$t$} \\
\hline & $\boldsymbol{h}_{m}$ & $S \%$ & $N$ & $\boldsymbol{G}$ & $d_{g}$ & $V$ & $N$ & $\boldsymbol{G}$ & $d_{g}$ & $V$ & $I c_{G}$ & $\operatorname{lm} m_{G}$ & $I c_{v}$ & $I m_{v}$ & \\
\hline 5 & 4.1 & 96.6 & 625 & 1.4 & 5.3 & 3 & \multirow{10}{*}{313} & \multirow{10}{*}{15.1} & \multirow{10}{*}{24.8} & \multirow{10}{*}{133} & 1.19 & 0.27 & 4.1 & 0.5 & 5 \\
\hline 10 & 10.1 & 39.8 & 625 & 10.6 & 14.7 & 52 & & & & & 1.81 & 1.06 & 13.3 & 5.2 & 10 \\
\hline 15 & 15.1 & 26.5 & 625 & 19.1 & 19.7 & 127 & & & & & 1.50 & 1.27 & 15.4 & 8.5 & 15 \\
\hline 20 & 18.9 & 21.2 & 625 & 25.5 & 22.8 & 201 & & & & & 1.10 & 1.27 & 13.7 & 10.0 & 20 \\
\hline 25 & 21.6 & 26.2 & 313 & 15.1 & 24.8 & 131 & & & & & 0.84 & 1.21 & 10.5 & 10.6 & 25 \\
\hline 30 & 23.5 & 24.1 & 313 & 18.9 & 27.7 & 174 & & & & & 0.67 & 1.13 & 8.0 & 10.2 & 30 \\
\hline 35 & 24.8 & 22.8 & 313 & 21.9 & 29.9 & 211 & & & & & 0.56 & 1.06 & 6.8 & 9.8 & 35 \\
\hline 40 & 25.7 & 22.0 & 313 & 24.5 & 31.6 & 242 & & & & & 0.48 & 0.99 & 5.8 & 9.4 & 40 \\
\hline 45 & 26.3 & 21.5 & 313 & 26.7 & 33.0 & 269 & & & & & 0.42 & 0.93 & 5.0 & 8.9 & 45 \\
\hline 50 & 26.7 & 21.2 & 313 & 28.7 & 34.2 & 293 & & & & & 0.38 & 0.88 & 4.4 & 8.5 & 50 \\
\hline 5 & 4.1 & 96.6 & 625 & 1.4 & 5.3 & 3 & \multirow{6}{*}{\multicolumn{4}{|c|}{$\begin{array}{l}\text { zonder dunning } \\
\text { without thinning }\end{array}$}} & 1.19 & 0.27 & 4.1 & 0.5 & 5 \\
\hline 10 & 10.1 & 39.8 & 625 & 10.6 & 14.7 & 52 & & & & & 1.81 & 1.06 & 13.3 & 5.2 & 10 \\
\hline 15 & 15.1 & 26.5 & 625 & 19.1 & 19.7 & 127 & & & & & 1.50 & 1.27 & 15.4 & 8.5 & 15 \\
\hline 20 & 18.9 & 21.2 & 625 & 25.5 & 22.8 & 201 & & & & & 1.10 & 1.27 & 13.7 & 10.0 & 20 \\
\hline 25 & 21.6 & 18.5 & 625 & 30.2 & 24.8 & 264 & & & & & 0.84 & 1.21 & 11.5 & 10.6 & 25 \\
\hline 29 & 23.2 & 17.3 & 625 & 33.3 & 26.0 & 306 & & & & & 0.70 & 1.15 & 9.8 & 10.6 & 29 \\
\hline
\end{tabular}




\begin{tabular}{|c|c|c|c|c|c|c|c|c|c|c|c|c|c|c|c|}
\hline \multicolumn{5}{|c|}{$\begin{array}{l}\text { Populier, plantverband } 4 \times 4 \mathrm{~m} \\
\text { Poplar, spacing } 4 \times 4 \mathrm{~m}\end{array}$} & \multicolumn{7}{|c|}{$\begin{array}{l}\text { met en zonder systematische } \\
\text { with and without systematically thinning }\end{array}$} & \multicolumn{4}{|c|}{$\begin{array}{r}\text { Boniteit V, } \boldsymbol{h}_{\mathbf{2 5}}=\mathbf{1 8 . 2} \\
\text { Site Class V, } h_{25}=18.2 \\
\end{array}$} \\
\hline \multirow[b]{2}{*}{$t$} & \multicolumn{6}{|c|}{$\begin{array}{l}\text { Kenmerken blijvende opstand } \\
\text { Characteristics remaining stand }\end{array}$} & \multicolumn{4}{|c|}{$\begin{array}{l}\text { met dunning } \\
\text { with thinning }\end{array}$} & \multicolumn{4}{|c|}{$\begin{array}{l}\text { Bijgroei } \\
\text { Increment }\end{array}$} & \multirow[b]{2}{*}{$t$} \\
\hline & $\boldsymbol{h}_{m}$ & S\% & $N$ & $\boldsymbol{G}$ & $d_{g}$ & $V$ & $N$ & $\boldsymbol{G}$ & $d_{g}$ & $V$ & $I c_{G}$ & $I m_{G}$ & $I c_{v}$ & $I m_{v}$ & \\
\hline 5 & 3.7 & 108.3 & 625 & 0.9 & 4.4 & 2 & \multirow{10}{*}{313} & \multirow{10}{*}{14.1} & \multirow{10}{*}{23.9} & \multirow{10}{*}{116} & 0.87 & 0.19 & 2.4 & 0.3 & 5 \\
\hline 10 & 8.6 & 46.5 & 625 & 8.2 & 12.9 & 36 & & & & & 1.46 & 0.82 & 9.8 & 3.6 & 10 \\
\hline 15 & 12.8 & 31.3 & 625 & 15.0 & 17.5 & 88 & & & & & 1.25 & 1.00 & 11.0 & 5.9 & 15 \\
\hline 20 & 15.9 & 25.1 & 625 & 20.6 & 20.5 & 143 & & & & & 0.97 & 1.03 & 10.3 & 7.1 & 20 \\
\hline 25 & 18.2 & 22.0 & 625 & 24.8 & 22.5 & 190 & & & & & 0.75 & 0.99 & 8.7 & 7.6 & 25 \\
\hline 30 & 19.8 & 28.6 & 313 & 14.1 & 23.9 & 114 & & & & & 0.60 & 0.94 & 6.7 & 7.7 & 30 \\
\hline 35 & 20.9 & 27.1 & 313 & 16.8 & 26.2 & 142 & & & & & 0.50 & 0.88 & 5.2 & 7.4 & 35 \\
\hline 40 & 21.6 & 26.2 & 313 & 19.1 & 27.9 & 166 & & & & & 0.43 & 0.83 & 4.5 & 7.0 & 40 \\
\hline 45 & 22.1 & 25.6 & 313 & 21.1 & 29.4 & 187 & & & & & 0.38 & 0.78 & 3.9 & 6.7 & 45 \\
\hline 50 & 22.4 & 25.2 & 313 & 23.0 & 30.6 & 205 & & & & & 0.34 & 0.74 & 3.5 & 6.4 & 50 \\
\hline 5 & 3.7 & 108.3 & 625 & 0.9 & 4.4 & 2 & \multirow{10}{*}{\multicolumn{4}{|c|}{$\begin{array}{l}\text { zonder dunning } \\
\text { without thinning }\end{array}$}} & 0.87 & 0.19 & 2.4 & 0.3 & 5 \\
\hline 10 & 8.6 & 46.5 & 625 & 8.2 & 12.9 & 36 & & & & & 1.46 & 0.82 & 9.8 & 3.6 & 10 \\
\hline 15 & 12.8 & 31.3 & 625 & 15.0 & 17.5 & 88 & & & & & 1.25 & 1.00 & 11.0 & 5.9 & 15 \\
\hline 20 & 15.9 & 25.1 & 625 & 20.6 & 20.5 & 143 & & & & & 0.97 & 1.03 & 10.3 & 7.1 & 20 \\
\hline 25 & 18.2 & 22.0 & 625 & 24.8 & 22.5 & 190 & & & & & 0.75 & 0.99 & 8.7 & 7.6 & 25 \\
\hline 30 & 19.8 & 20.2 & 625 & 28.1 & 23.9 & 230 & & & & & 0.60 & 0.94 & 7.2 & 7.7 & 30 \\
\hline 35 & 20.9 & 19.2 & 625 & 30.9 & 25.1 & 263 & & & & & 0.50 & 0.88 & 6.0 & 7.5 & 35 \\
\hline 40 & 21.6 & 18.5 & 625 & 33.2 & 26.0 & 290 & & & & & 0.43 & 0.83 & 5.0 & 7.2 & 40 \\
\hline 45 & 22.1 & 18.1 & 625 & 35.2 & 26.8 & 313 & & & & & 0.38 & 0.78 & 4.3 & 7.0 & 45 \\
\hline 50 & 22.4 & 17.8 & 625 & 37.0 & 27.5 & 333 & & & & & 0.34 & 0.74 & 3.7 & 6.7 & 50 \\
\hline
\end{tabular}




\begin{tabular}{|c|c|c|c|c|c|c|c|c|c|c|c|c|c|c|c|}
\hline \multirow{2}{*}{\multicolumn{7}{|c|}{$\begin{array}{l}\text { Populier, plantverband } \mathbf{5} \text { x } \mathbf{5} \text { m } \\
\text { Poplar, spacing } 5 \times 5 \mathrm{~m}\end{array}$}} & \multirow{2}{*}{\multicolumn{6}{|c|}{$\begin{array}{l}\text { met Flevoland beheer } \\
\text { with Flevoland management }\end{array}$}} & \multirow{2}{*}{\multicolumn{3}{|c|}{$\begin{array}{r}\text { Boniteit I, h25 = } \mathbf{3 1 . 8} \\
\text { Site Class I, h25 = 31.8 }\end{array}$}} \\
\hline & & & & & & & & & & & & & & & \\
\hline \multirow[b]{2}{*}{$t$} & \multicolumn{6}{|c|}{$\begin{array}{l}\text { Kenmerken blijvende opstand } \\
\text { Characteristics remaining stand }\end{array}$} & \multicolumn{4}{|c|}{$\begin{array}{l}\text { Dunning } \\
\text { Thinning }\end{array}$} & \multicolumn{4}{|c|}{$\begin{array}{l}\text { Bijgroei } \\
\text { Increment }\end{array}$} & \multirow[b]{2}{*}{$t$} \\
\hline & $\boldsymbol{h}_{m}$ & S\% & $N$ & $\boldsymbol{G}$ & $d_{g}$ & $v$ & $N$ & $\boldsymbol{G}$ & $d_{g}$ & $v$ & $I c_{G}$ & $\operatorname{Im}{ }_{G}$ & $I c_{V}$ & $I m_{V}$ & \\
\hline 5 & 5.5 & 91.2 & 400 & 2.4 & 8.8 & 6 & & & & & 1.67 & 0.49 & 8.2 & 1.2 & 5 \\
\hline 10 & 14.4 & 34.7 & 400 & 15.2 & 22.0 & 97 & & & & & 2.64 & 1.52 & 24.8 & 9.7 & 10 \\
\hline 12 & 17.7 & 40.0 & 200 & 10.2 & 25.5 & 76 & 200 & 10.2 & 25.5 & 76 & 2.39 & 1.70 & 25.0 & 12.7 & 12 \\
\hline 15 & 22.0 & 32.1 & 200 & 16.5 & 32.4 & 145 & & & & & 1.90 & 1.78 & 22.7 & 14.7 & 15 \\
\hline 17 & 24.5 & 40.8 & 100 & 10.1 & 35.8 & 96 & 100 & 10.1 & 35.8 & 96 & 1.65 & 1.78 & 20.6 & 15.7 & 17 \\
\hline 20 & 27.7 & 36.1 & 100 & 14.4 & 42.8 & 149 & & & & & 1.29 & 1.73 & 17.2 & 16.1 & 20 \\
\hline 25 & 31.8 & 31.4 & 100 & 20.1 & 50.6 & 232 & & & & & 1.04 & 1.61 & 15.8 & 16.2 & 25 \\
\hline
\end{tabular}

\begin{tabular}{|c|c|c|c|c|c|c|c|c|c|c|c|c|c|c|c|}
\hline \multirow{2}{*}{\multicolumn{7}{|c|}{$\begin{array}{l}\text { Populier, plantverband } 5 \text { x } 5 \text { m } \\
\text { Poplar, spacing } 5 \text { × } 5 \mathrm{~m}\end{array}$}} & \multirow{2}{*}{\multicolumn{6}{|c|}{$\begin{array}{l}\text { met Flevoland beheer } \\
\text { with Flevoland management }\end{array}$}} & \multirow{2}{*}{\multicolumn{3}{|c|}{$\begin{array}{r}\text { Boniteit II, h25 = } \mathbf{2 8 . 4} \\
\text { Site Class II, h25 = 28.4 }\end{array}$}} \\
\hline & & & & & & & & & & & & & & & \\
\hline \multirow[b]{2}{*}{$t$} & \multicolumn{6}{|c|}{$\begin{array}{l}\text { Kenmerken blijvende opstand } \\
\text { Characteristics remaining stand }\end{array}$} & \multicolumn{4}{|c|}{$\begin{array}{l}\text { Dunning } \\
\text { Thinning }\end{array}$} & \multicolumn{4}{|c|}{$\begin{array}{l}\text { Bijgroei } \\
\text { Increment }\end{array}$} & \multirow[b]{2}{*}{$t$} \\
\hline & $h_{m}$ & S\% & $N$ & $\boldsymbol{G}$ & $d_{g}$ & $v$ & $N$ & $\boldsymbol{G}$ & $d_{g}$ & $v$ & $I c_{G}$ & $\operatorname{Im}{ }_{G}$ & $I c_{V}$ & $I m_{V}$ & \\
\hline 5 & 5.0 & 99.3 & 400 & 1.9 & 7.8 & 4 & & & & & 1.39 & 0.38 & 6.3 & 0.9 & 5 \\
\hline 10 & 12.9 & 38.6 & 400 & 12.7 & 20.1 & 75 & & & & & 2.26 & 1.27 & 19.7 & 7.5 & 10 \\
\hline 14 & 18.5 & 38.3 & 200 & 10.7 & 26.1 & 83 & 200 & 10.7 & 26.1 & 83 & 1.92 & 1.53 & 20.9 & 11.8 & 14 \\
\hline 15 & 19.7 & 35.9 & 200 & 12.6 & 28.4 & 102 & & & & & 1.78 & 1.56 & 19.8 & 12.3 & 15 \\
\hline 20 & 24.8 & 28.5 & 200 & 20.0 & 35.6 & 192 & & & & & 1.30 & 1.53 & 16.6 & 13.7 & 20 \\
\hline 21 & 25.6 & 39.1 & 100 & 10.6 & 36.8 & 105 & 100 & 10.6 & 36.8 & 105 & 1.21 & 1.52 & 15.4 & 13.9 & 21 \\
\hline 25 & 28.4 & 35.2 & 100 & 14.7 & 43.3 & 156 & & & & & 0.90 & 1.44 & 12.1 & 13.7 & 25 \\
\hline
\end{tabular}




\begin{tabular}{|c|c|c|c|c|c|c|c|c|c|c|c|c|c|c|c|}
\hline \multirow{2}{*}{\multicolumn{7}{|c|}{$\begin{array}{l}\text { Populier, plantverband } 5 \text { x } 5 \text { m } \\
\text { Poplar, spacing } 5 \times 5 \mathrm{~m}\end{array}$}} & \multirow{2}{*}{\multicolumn{6}{|c|}{$\begin{array}{l}\text { met Flevoland beheer } \\
\text { with Flevoland management }\end{array}$}} & \multirow{2}{*}{\multicolumn{3}{|c|}{$\begin{array}{r}\text { Boniteit III, h25 = } \mathbf{2 5 . 0} \\
\text { Site Class III, h25 = 25.0 }\end{array}$}} \\
\hline & & & & & & & & & & & & & & & \\
\hline \multirow[b]{2}{*}{$t$} & \multicolumn{6}{|c|}{$\begin{array}{l}\text { Kenmerken blijvende opstand } \\
\text { Characteristics remaining stand }\end{array}$} & \multicolumn{4}{|c|}{$\begin{array}{l}\text { Dunning } \\
\text { Thinning }\end{array}$} & \multicolumn{4}{|c|}{$\begin{array}{l}\text { Bijgroei } \\
\text { Increment }\end{array}$} & \multirow[b]{2}{*}{$t$} \\
\hline & $h_{m}$ & S\% & $N$ & $G$ & $d_{g}$ & $V$ & $N$ & $\boldsymbol{G}$ & $d_{g}$ & $v$ & $I c_{G}$ & $I m_{G}$ & $I c_{v}$ & $I m_{v}$ & \\
\hline 5 & 4.6 & 109.0 & 400 & 1.4 & 6.8 & 3 & & & & & 1.14 & 0.29 & 4.7 & 0.6 & 5 \\
\hline 10 & 11.5 & 43.5 & 400 & 10.4 & 18.2 & 56 & & & & & 1.88 & 1.04 & 14.9 & 5.6 & 10 \\
\hline 15 & 17.4 & 28.7 & 400 & 19.4 & 24.9 & 144 & & & & & 1.62 & 1.29 & 17.2 & 9.6 & 15 \\
\hline 16 & 18.4 & 38.4 & 200 & 10.5 & 25.9 & 81 & 200 & 10.5 & 25.9 & 81 & 1.51 & 1.32 & 16.3 & 10.1 & 16 \\
\hline 20 & 21.8 & 32.4 & 200 & 15.6 & 31.5 & 136 & & & & & 1.13 & 1.31 & 13.3 & 10.9 & 20 \\
\hline 25 & 25.0 & 40.0 & 200 & 20.7 & 36.3 & 201 & & & & & 0.89 & 1.25 & 10.8 & 11.3 & 25 \\
\hline
\end{tabular}

\begin{tabular}{|c|c|c|c|c|c|c|c|c|c|c|c|c|c|c|c|}
\hline \multirow{2}{*}{\multicolumn{7}{|c|}{$\begin{array}{l}\text { Populier, plantverband } 5 \text { x } 5 \text { m } \\
\text { Poplar, spacing } 5 \times 5 \mathrm{~m}\end{array}$}} & \multirow{2}{*}{\multicolumn{6}{|c|}{$\begin{array}{l}\text { met Flevoland beheer } \\
\text { with Flevoland management }\end{array}$}} & \multirow{2}{*}{\multicolumn{3}{|c|}{$\begin{array}{r}\text { Boniteit IV, h25 = } \mathbf{2 1 . 6} \\
\text { Site Class IV, h25 = } 21.6\end{array}$}} \\
\hline & & & & & & & & & & & & & & & \\
\hline \multirow[b]{2}{*}{$t$} & \multicolumn{6}{|c|}{$\begin{array}{l}\text { Kenmerken blijvende opstand } \\
\text { Characteristics remaining stand }\end{array}$} & \multicolumn{4}{|c|}{$\begin{array}{l}\text { Dunning } \\
\text { Thinning }\end{array}$} & \multicolumn{4}{|c|}{$\begin{array}{l}\text { Bijgroei } \\
\text { Increment }\end{array}$} & \multirow[b]{2}{*}{$t$} \\
\hline & $\boldsymbol{h}_{m}$ & S\% & $N$ & $\boldsymbol{G}$ & $d_{g}$ & $V$ & $N$ & $\boldsymbol{G}$ & $d_{g}$ & $v$ & $I c_{G}$ & $\operatorname{Im}{ }_{G}$ & $I c_{V}$ & $\operatorname{Im}{ }_{V}$ & \\
\hline 5 & 4.1 & 120.7 & 400 & 1.0 & 5.7 & 2 & & & & & 0.89 & 0.21 & 3.1 & 0.4 & 5 \\
\hline 10 & 10.1 & 49.7 & 400 & 8.2 & 16.2 & 40 & & & & & 1.51 & 0.82 & 10.9 & 4.0 & 10 \\
\hline 15 & 15.1 & 33.1 & 400 & 15.6 & 22.3 & 104 & & & & & 1.37 & 1.04 & 13.6 & 6.9 & 15 \\
\hline 19 & 18.2 & 38.8 & 200 & 10.4 & 25.7 & 79 & 200 & 10.4 & 25.7 & 79 & 1.12 & 1.09 & 11.8 & 8.3 & 19 \\
\hline 20 & 18.9 & 37.4 & 200 & 11.5 & 27.0 & 90 & & & & & 1.03 & 1.09 & 10.9 & 8.4 & 20 \\
\hline 25 & 21.6 & 32.7 & 200 & 15.7 & 31.7 & 136 & & & & & 0.78 & 1.04 & 8.9 & 8.6 & 25 \\
\hline 30 & 23.5 & 30.1 & 200 & 19.3 & 35.1 & 178 & & & & & 0.65 & 0.99 & 7.7 & 8.6 & 30 \\
\hline
\end{tabular}




\begin{tabular}{|c|c|c|c|c|c|c|c|c|c|c|c|c|c|c|c|}
\hline \multirow{2}{*}{\multicolumn{7}{|c|}{$\begin{array}{l}\text { Populier, plantverband } \mathbf{5} \times \mathbf{5} \mathbf{~ m} \\
\text { Poplar, spacing } 5 \times 5 \mathrm{~m}\end{array}$}} & \multirow{2}{*}{\multicolumn{6}{|c|}{$\begin{array}{l}\text { met Flevoland beheer } \\
\text { with Flevoland management }\end{array}$}} & \multirow{2}{*}{\multicolumn{3}{|c|}{$\begin{array}{r}\text { Boniteit V, h25 = } \mathbf{1 8 . 2} \\
\text { Site Class V, h25 = } 18.2\end{array}$}} \\
\hline & & & & & & & & & & & & & & & \\
\hline \multirow[b]{2}{*}{$t$} & \multicolumn{6}{|c|}{$\begin{array}{l}\text { Kenmerken blijvende opstand } \\
\text { Characteristics remaining stand }\end{array}$} & \multicolumn{4}{|c|}{$\begin{array}{l}\text { Dunning } \\
\text { Thinning }\end{array}$} & \multicolumn{4}{|c|}{$\begin{array}{l}\text { Bijgroei } \\
\text { Increment }\end{array}$} & \multirow[b]{2}{*}{$t$} \\
\hline & $\boldsymbol{h}_{m}$ & S\% & $N$ & $G$ & $d_{g}$ & $v$ & $N$ & $\boldsymbol{G}$ & $d_{g}$ & $v$ & $I c_{G}$ & $I m_{G}$ & $I c_{V}$ & $I m_{V}$ & \\
\hline 5 & 3.7 & 135.3 & 400 & 0.7 & 4.7 & 1 & & & & & 0.65 & 0.14 & 1.8 & 0.3 & 5 \\
\hline 10 & 8.6 & 58.1 & 400 & 6.3 & 14.1 & 27 & & & & & 1.17 & 0.63 & 7.7 & 2.7 & 10 \\
\hline 15 & 12.8 & 39.1 & 400 & 12.1 & 19.6 & 71 & & & & & 1.09 & 0.80 & 9.4 & 4.7 & 15 \\
\hline 20 & 15.9 & 31.4 & 400 & 17.1 & 23.3 & 118 & & & & & 0.91 & 0.85 & 9.3 & 5.9 & 20 \\
\hline 24 & 17.8 & 39.7 & 200 & 10.2 & 25.5 & 77 & 200 & 10.2 & 25.5 & 77 & 0.75 & 0.85 & 7.5 & 6.4 & 24 \\
\hline 25 & 18.2 & 38.9 & 200 & 11.0 & 26.5 & 83 & & & & & 0.70 & 0.85 & 6.9 & 6.4 & 25 \\
\hline 30 & 19.8 & 35.8 & 200 & 13.9 & 29.7 & 112 & & & & & 0.53 & 0.80 & 5.4 & 6.3 & 30 \\
\hline
\end{tabular}




\begin{tabular}{|c|c|c|c|c|c|c|c|c|c|c|c|c|c|c|c|}
\hline \multirow{2}{*}{\multicolumn{7}{|c|}{$\begin{array}{l}\text { Populier, plantverband } 5 \text { x } 6 \text { m } \\
\text { Poplar, spacing } 5 \times 6 \mathrm{~m}\end{array}$}} & \multirow{2}{*}{\multicolumn{6}{|c|}{$\begin{array}{l}\text { met Flevoland beheer } \\
\text { with Flevoland management }\end{array}$}} & \multirow{2}{*}{\multicolumn{3}{|c|}{$\begin{array}{r}\text { Boniteit I, h25 = } \mathbf{3 1 . 8} \\
\text { Site Class I, h25 = 31.8 }\end{array}$}} \\
\hline & & & & & & & & & & & & & & & \\
\hline \multirow[b]{2}{*}{$t$} & \multicolumn{6}{|c|}{$\begin{array}{l}\text { Kenmerken blijvende opstand } \\
\text { Characteristics remaining stand }\end{array}$} & \multicolumn{4}{|c|}{$\begin{array}{l}\text { Dunning } \\
\text { Thinning }\end{array}$} & \multicolumn{4}{|c|}{$\begin{array}{l}\text { Bijgroei } \\
\text { Increment }\end{array}$} & \multirow[b]{2}{*}{$t$} \\
\hline & $\boldsymbol{h}_{m}$ & S\% & $N$ & $\boldsymbol{G}$ & $d_{g}$ & $v$ & $N$ & $\boldsymbol{G}$ & $d_{g}$ & $v$ & $I c_{G}$ & $\operatorname{Im}{ }_{G}$ & $I c_{V}$ & $I m_{V}$ & \\
\hline 5 & 5.5 & 99.9 & 333 & 2.2 & 9.1 & 5 & & & & & 1.49 & 0.44 & 7.2 & 1.1 & 5 \\
\hline 10 & 14.4 & 38.1 & 333 & 13.8 & 22.9 & 88 & & & & & 2.49 & 1.38 & 23.5 & 8.8 & 10 \\
\hline 13 & 19.2 & 40.4 & 167 & 10.5 & 28.4 & 84 & 167 & 10.5 & 28.4 & 84 & 2.20 & 1.62 & 24.4 & 12.9 & 13 \\
\hline 15 & 22.0 & 35.2 & 167 & 14.6 & 33.4 & 128 & & & & & 1.89 & 1.68 & 22.4 & 14.1 & 15 \\
\hline 19 & 26.7 & 41.0 & 83 & 10.6 & 40.2 & 108 & 83 & 10.6 & 40.2 & 108 & 1.45 & 1.67 & 19.2 & 15.7 & 19 \\
\hline 20 & 27.7 & 39.5 & 83 & 12.1 & 43.0 & 125 & & & & & 1.34 & 1.66 & 17.9 & 15.8 & 20 \\
\hline 25 & 31.8 & 34.4 & 83 & 17.5 & 51.7 & 202 & & & & & 0.99 & 1.55 & 14.7 & 15.7 & 25 \\
\hline
\end{tabular}

\begin{tabular}{|c|c|c|c|c|c|c|c|c|c|c|c|c|c|c|c|}
\hline \multirow{2}{*}{\multicolumn{7}{|c|}{$\begin{array}{l}\text { Populier, plantverband } 5 \text { × } 6 \text { m } \\
\text { Poplar, spacing } 5 \times 6 \mathrm{~m}\end{array}$}} & \multirow{2}{*}{\multicolumn{6}{|c|}{$\begin{array}{l}\text { met Flevoland beheer } \\
\text { with Flevoland management }\end{array}$}} & \multirow{2}{*}{\multicolumn{3}{|c|}{$\begin{array}{r}\text { Boniteit II, h25 = } \mathbf{2 8 . 4} \\
\text { Site Class II, h25 = 28.4 }\end{array}$}} \\
\hline & & & & & & & & & & & & & & & \\
\hline \multirow[b]{2}{*}{$t$} & \multicolumn{6}{|c|}{$\begin{array}{l}\text { Kenmerken blijvende opstand } \\
\text { Characteristics remaining stand }\end{array}$} & \multicolumn{4}{|c|}{$\begin{array}{l}\text { Dunning } \\
\text { Thinning }\end{array}$} & \multicolumn{4}{|c|}{$\begin{array}{l}\text { Bijgroei } \\
\text { Increment }\end{array}$} & \multirow[b]{2}{*}{$t$} \\
\hline & $\boldsymbol{h}_{m}$ & S\% & $N$ & $\boldsymbol{G}$ & $d_{g}$ & $V$ & $N$ & $\boldsymbol{G}$ & $d_{g}$ & $V$ & $I c_{G}$ & $I m_{G}$ & $I c_{v}$ & $I m_{V}$ & \\
\hline 5 & 5.0 & 108.8 & 333 & 1.7 & 8.1 & 4 & & & & & 1.24 & 0.34 & 5.6 & 0.8 & 5 \\
\hline 10 & 12.9 & 42.3 & 333 & 11.5 & 20.9 & 68 & & & & & 2.11 & 1.15 & 18.3 & 6.8 & 10 \\
\hline 15 & 19.7 & 39.3 & 167 & 10.8 & 28.7 & 88 & 167 & 10.8 & 28.7 & 88 & 1.76 & 1.44 & 20.0 & 11.7 & 15 \\
\hline 20 & 24.8 & 31.3 & 167 & 18.1 & 37.1 & 173 & & & & & 1.26 & 1.44 & 16.3 & 13.0 & 20 \\
\hline 22 & 26.4 & 41.5 & 83 & 10.2 & 39.6 & 103 & 83 & 10.2 & 39.6 & 103 & 1.11 & 1.42 & 14.3 & 13.4 & 22 \\
\hline 25 & 28.4 & 38.6 & 83 & 13.2 & 44.8 & 139 & & & & & 0.87 & 1.37 & 11.4 & 13.2 & 25 \\
\hline
\end{tabular}




\begin{tabular}{|c|c|c|c|c|c|c|c|c|c|c|c|c|c|c|c|}
\hline \multirow{2}{*}{\multicolumn{7}{|c|}{$\begin{array}{l}\text { Populier, plantverband } 5 \text { × } 6 \text { m } \\
\text { Poplar, spacing } 5 \times 6 \mathrm{~m}\end{array}$}} & \multirow{2}{*}{\multicolumn{6}{|c|}{$\begin{array}{l}\text { met Flevoland beheer } \\
\text { with Flevoland management }\end{array}$}} & \multirow{2}{*}{\multicolumn{3}{|c|}{$\begin{array}{r}\text { Boniteit III, h25 = } \mathbf{2 5 . 0} \\
\text { Site Class III, h25 = 25.0 }\end{array}$}} \\
\hline & & & & & & & & & & & & & & & \\
\hline \multirow[b]{2}{*}{$t$} & \multicolumn{6}{|c|}{$\begin{array}{l}\text { Kenmerken blijvende opstand } \\
\text { Characteristics remaining stand }\end{array}$} & \multicolumn{4}{|c|}{$\begin{array}{l}\text { Dunning } \\
\text { Thinning }\end{array}$} & \multicolumn{4}{|c|}{$\begin{array}{l}\text { Bijgroei } \\
\text { Increment }\end{array}$} & \multirow[b]{2}{*}{$t$} \\
\hline & $h_{m}$ & S\% & $N$ & $\boldsymbol{G}$ & $d_{g}$ & $V$ & $N$ & $\boldsymbol{G}$ & $d_{g}$ & $v$ & $I c_{G}$ & $I m_{G}$ & $I c_{v}$ & $I m_{v}$ & \\
\hline 5 & 4.6 & 119.4 & 333 & 1.3 & 7.0 & 3 & & & & & 1.01 & 0.26 & 4.2 & 0.5 & 5 \\
\hline 10 & 11.5 & 47.6 & 333 & 9.3 & 18.9 & 50 & & & & & 1.74 & 0.93 & 13.6 & 5.0 & 10 \\
\hline 15 & 17.4 & 31.5 & 333 & 17.9 & 26.1 & 132 & & & & & 1.57 & 1.19 & 17.1 & 8.8 & 15 \\
\hline 17 & 19.3 & 40.1 & 167 & 10.5 & 28.3 & 84 & 167 & 10.5 & 28.3 & 84 & 1.39 & 1.23 & 15.4 & 9.8 & 17 \\
\hline 20 & 21.8 & 35.5 & 167 & 14.1 & 32.8 & 123 & & & & & 1.09 & 1.23 & 12.6 & 10.3 & 20 \\
\hline 25 & 25.0 & 31.0 & 167 & 19.0 & 38.1 & 183 & & & & & 0.89 & 1.18 & 11.3 & 10.7 & 25 \\
\hline
\end{tabular}

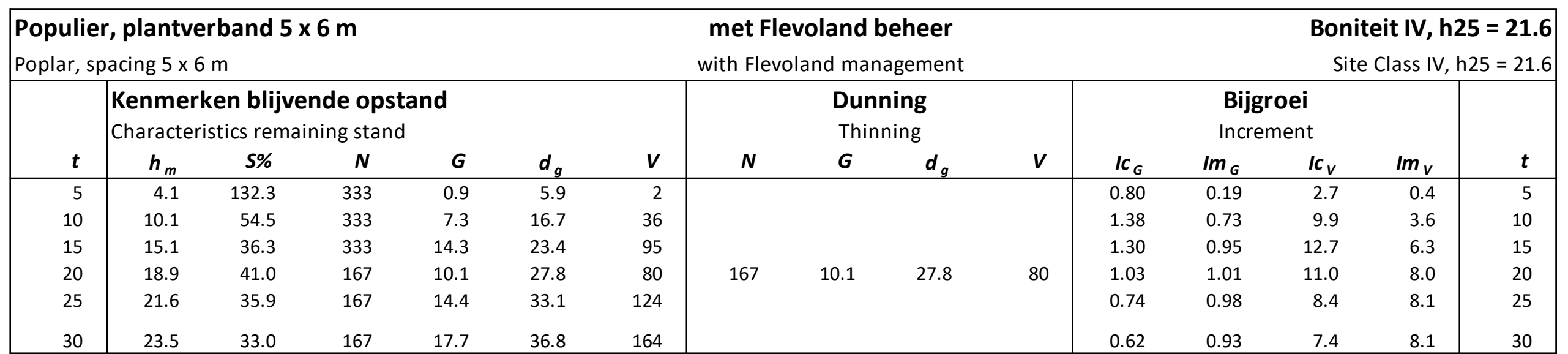




\begin{tabular}{|c|c|c|c|c|c|c|c|c|c|c|c|c|c|c|c|}
\hline \multirow{2}{*}{\multicolumn{7}{|c|}{$\begin{array}{l}\text { Populier, plantverband } 5 \times 6 \text { m } \\
\text { Poplar, spacing } 5 \times 6 \mathrm{~m}\end{array}$}} & \multirow{2}{*}{\multicolumn{6}{|c|}{$\begin{array}{l}\text { met Flevoland beheer } \\
\text { with Flevoland management }\end{array}$}} & \multirow{2}{*}{\multicolumn{3}{|c|}{$\begin{array}{r}\text { Boniteit V, h25 = } \mathbf{1 8 . 2} \\
\text { Site Class V, h25 = } 18.2\end{array}$}} \\
\hline & & & & & & & & & & & & & & & \\
\hline \multirow[b]{2}{*}{$t$} & \multicolumn{6}{|c|}{$\begin{array}{l}\text { Kenmerken blijvende opstand } \\
\text { Characteristics remaining stand }\end{array}$} & \multicolumn{4}{|c|}{$\begin{array}{l}\text { Dunning } \\
\text { Thinning }\end{array}$} & \multicolumn{4}{|c|}{$\begin{array}{l}\text { Bijgroei } \\
\text { Increment }\end{array}$} & \multirow[b]{2}{*}{$t$} \\
\hline & $\boldsymbol{h}_{m}$ & S\% & $N$ & $G$ & $d_{g}$ & $v$ & $N$ & $\boldsymbol{G}$ & $d_{g}$ & $v$ & $I c_{G}$ & $I m_{G}$ & $I c_{V}$ & $I m_{V}$ & \\
\hline 5 & 3.7 & 148.3 & 333 & 0.6 & 4.9 & 1 & & & & & 0.58 & 0.13 & 1.6 & 0.2 & 5 \\
\hline 10 & 8.6 & 63.6 & 333 & 5.6 & 14.6 & 24 & & & & & 1.05 & 0.56 & 6.9 & 2.4 & 10 \\
\hline 15 & 12.8 & 42.8 & 333 & 10.9 & 20.4 & 64 & & & & & 1.03 & 0.73 & 8.7 & 4.3 & 15 \\
\hline 20 & 15.9 & 34.3 & 333 & 15.7 & 24.5 & 108 & & & & & 0.87 & 0.78 & 8.7 & 5.4 & 20 \\
\hline 25 & 18.2 & 30.1 & 333 & 19.6 & 27.4 & 150 & & & & & 0.71 & 0.79 & 7.3 & 6.0 & 25 \\
\hline 26 & 18.6 & 41.7 & 167 & 10.2 & 27.9 & 79 & 167 & 10.2 & 27.9 & 79 & 0.67 & 0.78 & 6.7 & 6.1 & 26 \\
\hline 30 & 19.8 & 39.2 & 167 & 12.4 & 30.8 & 101 & & & & & 0.50 & 0.75 & 5.1 & 6.0 & 30 \\
\hline
\end{tabular}




\section{Lijnbeplantingen van Populier}

Poplar line plantations

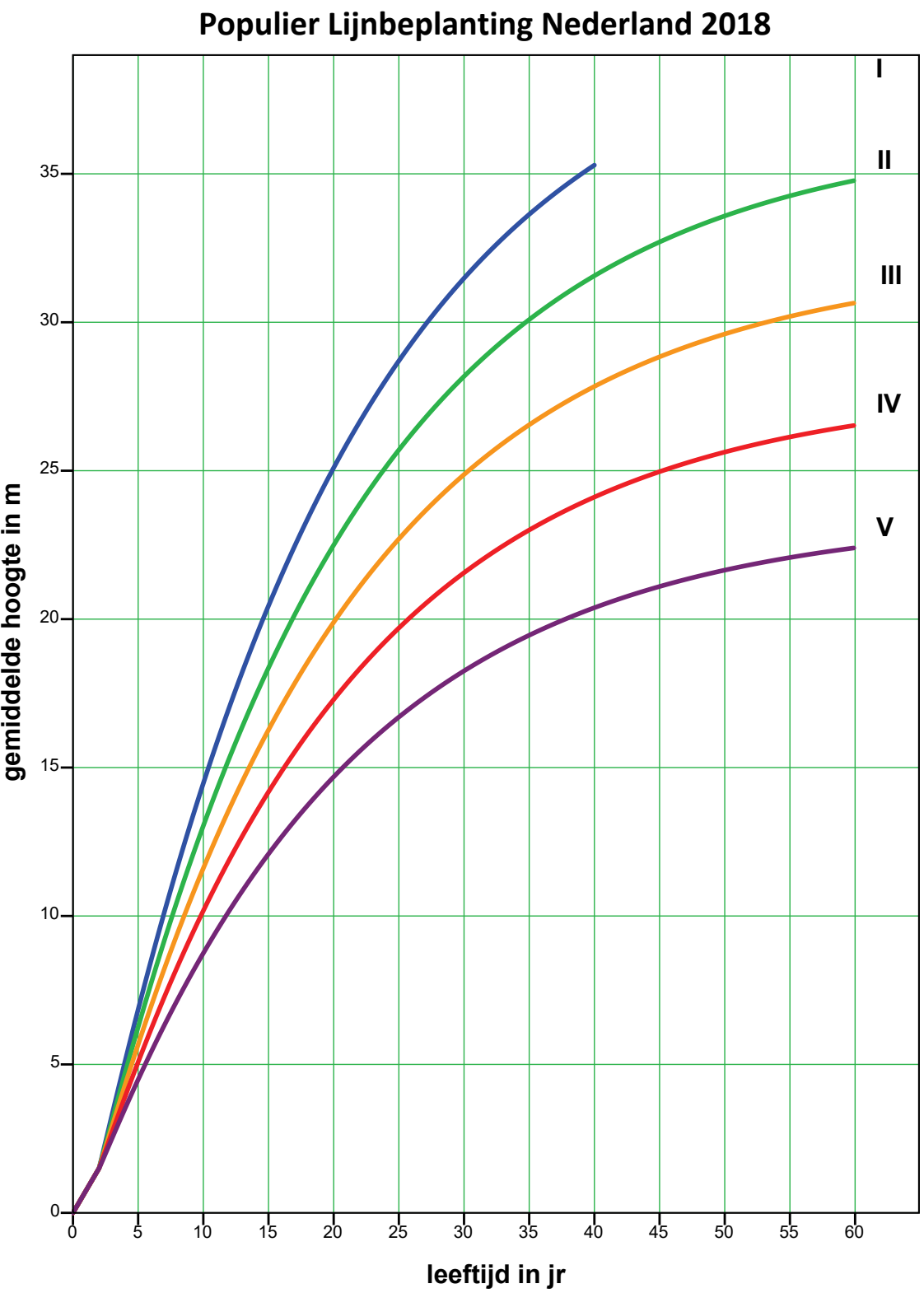




\begin{tabular}{|c|c|c|c|c|c|c|c|c|c|c|c|c|c|}
\hline \multirow{2}{*}{\multicolumn{3}{|c|}{$\begin{array}{l}\text { Populier, Nederland } 2018 \\
\text { Poplar, Netherlands } 2018\end{array}$}} & \multicolumn{8}{|c|}{ lijnbeplanting met plantafstand $3.2 \mathrm{~m}$, zonder dunning } & \multirow{2}{*}{\multicolumn{3}{|c|}{$\begin{array}{r}\text { Boniteit I, } \boldsymbol{h}_{\mathbf{2 5}}=\mathbf{2 8 . 7} \\
\text { Site Class I, } h_{25}=28.7\end{array}$}} \\
\hline & & & & & ntatio & h spa & $3.2 \mathrm{~m}$ & nout $t$ & & & & & \\
\hline \multirow[b]{2}{*}{$t$} & \multicolumn{8}{|c|}{$\begin{array}{l}\text { Kenmerken blijvende opstand } \\
\text { Characteristics remaining stand }\end{array}$} & \multicolumn{4}{|c|}{$\begin{array}{l}\text { Bijgroei } \\
\text { Increment }\end{array}$} & \multirow[b]{2}{*}{$t$} \\
\hline & $h_{m}$ & S\% & $N$ & $\boldsymbol{G}$ & $d_{g}$ & $d_{\text {or }}$ & $\boldsymbol{d}_{i r}$ & $v$ & $I c_{G}$ & $I m_{G}$ & $I c_{v}$ & $I m_{V}$ & \\
\hline 5 & 6.9 & 46.7 & 313 & 3.6 & 12.2 & 12.3 & 12.0 & 11 & 1.64 & 0.73 & 8.8 & 2.1 & 5 \\
\hline 10 & 14.5 & 22.1 & 313 & 14.5 & 24.3 & 24.6 & 23.9 & 93 & 2.65 & 1.45 & 23.6 & 9.3 & 10 \\
\hline 15 & 20.4 & 15.6 & 313 & 28.5 & 34.1 & 34.6 & 33.6 & 239 & 2.89 & 1.90 & 33.6 & 15.9 & 15 \\
\hline 20 & 25.1 & 12.7 & 313 & 42.8 & 41.8 & 42.4 & 41.1 & 419 & 2.78 & 2.14 & 37.6 & 21.0 & 20 \\
\hline 25 & 28.7 & 11.1 & 313 & 56.1 & 47.8 & 48.6 & 47.1 & 608 & 2.51 & 2.24 & 37.5 & 24.3 & 25 \\
\hline 30 & 31.5 & 10.2 & 313 & 67.9 & 52.6 & 53.4 & 51.8 & 790 & 2.19 & 2.26 & 34.9 & 26.3 & 30 \\
\hline 35 & 33.6 & 9.5 & 313 & 78.0 & 56.4 & 57.3 & 55.5 & 955 & 1.87 & 2.23 & 31.0 & 27.3 & 35 \\
\hline 40 & 35.3 & 9.1 & 313 & 86.6 & 59.4 & 60.3 & 58.5 & 1100 & 1.56 & 2.16 & 26.8 & 27.5 & 40 \\
\hline
\end{tabular}




\begin{tabular}{|c|c|c|c|c|c|c|c|c|c|c|c|c|c|}
\hline \multirow{2}{*}{\multicolumn{3}{|c|}{$\begin{array}{l}\text { Populier, Nederland } 2018 \\
\text { Poplar, Netherlands } 2018\end{array}$}} & \multicolumn{8}{|c|}{ lijnbeplanting met plantafstand $3.2 \mathrm{~m}$, zonder dunning } & \multirow{2}{*}{\multicolumn{3}{|c|}{$\begin{array}{r}\text { Boniteit II, } \boldsymbol{h}_{\mathbf{2 5}}=\mathbf{2 5 . 7} \\
\text { Site Class II, } h_{25}=25.7\end{array}$}} \\
\hline & & & & $\operatorname{lin} \epsilon$ & ntatic & th $\mathrm{sp}$ & $3.2 \mathrm{~m}$ & hout $t$ & & & & & \\
\hline \multirow[b]{2}{*}{$t$} & \multicolumn{8}{|c|}{$\begin{array}{l}\text { Kenmerken blijvende opstand } \\
\text { Characteristics remaining stand }\end{array}$} & \multicolumn{4}{|c|}{$\begin{array}{l}\text { Bijgroei } \\
\text { Increment }\end{array}$} & \multirow[b]{2}{*}{$t$} \\
\hline & $\boldsymbol{h}_{m}$ & S\% & $N$ & $\boldsymbol{G}$ & $d_{g}$ & $d_{\text {or }}$ & $\boldsymbol{d}_{i r}$ & $v$ & $I c_{G}$ & $I m_{G}$ & $I c_{v}$ & $I m_{v}$ & \\
\hline 5 & 6.3 & 51.1 & 313 & 3.0 & 11.0 & 11.1 & 10.8 & 8 & 1.20 & 0.59 & 6.0 & 1.6 & 5 \\
\hline 10 & 13.0 & 24.5 & 313 & 10.8 & 21.0 & 21.4 & 20.6 & 64 & 1.98 & 1.08 & 16.3 & 6.4 & 10 \\
\hline 15 & 18.4 & 17.4 & 313 & 21.3 & 29.5 & 30.1 & 28.9 & 164 & 2.16 & 1.42 & 23.0 & 11.0 & 15 \\
\hline 20 & 22.5 & 14.2 & 313 & 32.0 & 36.1 & 36.9 & 35.4 & 288 & 2.08 & 1.60 & 25.8 & 14.4 & 20 \\
\hline 25 & 25.7 & 12.5 & 313 & 41.9 & 41.3 & 42.2 & 40.4 & 418 & 1.88 & 1.68 & 25.7 & 16.7 & 25 \\
\hline 30 & 28.2 & 11.4 & 313 & 50.7 & 45.5 & 46.5 & 44.5 & 542 & 1.64 & 1.69 & 23.9 & 18.1 & 30 \\
\hline 35 & 30.1 & 10.6 & 313 & 58.3 & 48.7 & 49.8 & 47.7 & 655 & 1.39 & 1.67 & 21.3 & 18.7 & 35 \\
\hline 40 & 31.6 & 10.1 & 313 & 64.7 & 51.3 & 52.5 & 50.2 & 755 & 1.17 & 1.62 & 18.4 & 18.9 & 40 \\
\hline 45 & 32.7 & 9.8 & 313 & 70.0 & 53.4 & 54.6 & 52.2 & 839 & 0.97 & 1.56 & 15.5 & 18.6 & 45 \\
\hline 50 & 33.6 & 9.5 & 313 & 74.4 & 55.1 & 56.3 & 53.9 & 910 & 0.80 & 1.49 & 12.9 & 18.2 & 50 \\
\hline 55 & 34.3 & 9.3 & 313 & 78.0 & 56.4 & 57.7 & 55.1 & 969 & 0.65 & 1.42 & 10.6 & 17.6 & 55 \\
\hline 60 & 34.8 & 9.2 & 313 & 81.0 & 57.4 & 58.7 & 56.2 & 1017 & 0.53 & 1.35 & 8.7 & 17.0 & 60 \\
\hline
\end{tabular}




\begin{tabular}{|c|c|c|c|c|c|c|c|c|c|c|c|c|c|}
\hline \multirow{2}{*}{\multicolumn{3}{|c|}{$\begin{array}{l}\text { Populier, Nederland } 2018 \\
\text { Poplar, Netherlands } 2018\end{array}$}} & \multicolumn{8}{|c|}{ lijnbeplanting met plantafstand $3.2 \mathrm{~m}$, zonder dunning } & \multirow{2}{*}{\multicolumn{3}{|c|}{$\begin{array}{r}\text { Boniteit III, } \boldsymbol{h}_{\mathbf{2 5}}=\mathbf{2 2 . 7} \\
\text { Site Class III, } h_{25}=22.7\end{array}$}} \\
\hline & & & & line & ntatio & th spa & $3.2 \mathrm{~m}$, & out $\mathrm{t}$ & & & & & \\
\hline \multirow[b]{2}{*}{$t$} & \multicolumn{8}{|c|}{$\begin{array}{l}\text { Kenmerken blijvende opstand } \\
\text { Characteristics remaining stand }\end{array}$} & \multicolumn{4}{|c|}{$\begin{array}{l}\text { Bijgroei } \\
\text { Increment }\end{array}$} & \multirow[b]{2}{*}{$t$} \\
\hline & $h_{m}$ & S\% & $N$ & $\boldsymbol{G}$ & $d_{g}$ & $d_{o r}$ & $\boldsymbol{d}_{i r}$ & $v$ & $I c_{G}$ & $I m_{G}$ & $I c_{v}$ & $I m_{V}$ & \\
\hline 5 & 5.7 & 56.4 & 313 & 2.3 & 9.7 & 9.9 & 9.5 & 6 & 1.37 & 0.46 & 4.6 & 1.1 & 5 \\
\hline 10 & 11.6 & 27.6 & 313 & 7.9 & 17.9 & 18.4 & 17.5 & 43 & 1.44 & 0.79 & 10.8 & 4.3 & 10 \\
\hline 15 & 16.3 & 19.7 & 313 & 15.5 & 25.2 & 25.8 & 24.5 & 109 & 1.57 & 1.04 & 15.2 & 7.3 & 15 \\
\hline 20 & 19.9 & 16.1 & 313 & 23.3 & 30.8 & 31.7 & 30.0 & 191 & 1.51 & 1.17 & 17.0 & 9.5 & 20 \\
\hline 25 & 22.7 & 14.1 & 313 & 30.5 & 35.3 & 36.3 & 34.3 & 276 & 1.37 & 1.22 & 17.0 & 11.1 & 25 \\
\hline 30 & 24.9 & 12.9 & 313 & 37.0 & 38.8 & 39.9 & 37.7 & 359 & 1.19 & 1.23 & 15.8 & 12.0 & 30 \\
\hline 35 & 26.5 & 12.1 & 313 & 42.5 & 41.6 & 42.8 & 40.4 & 433 & 1.02 & 1.21 & 14.0 & 12.4 & 35 \\
\hline 40 & 27.8 & 11.5 & 313 & 47.1 & 43.8 & 45.1 & 42.6 & 499 & 0.85 & 1.18 & 12.1 & 12.5 & 40 \\
\hline 45 & 28.8 & 11.1 & 313 & 51.0 & 45.6 & 46.9 & 44.3 & 555 & 0.71 & 1.13 & 10.2 & 12.3 & 45 \\
\hline 50 & 29.6 & 10.8 & 313 & 54.2 & 47.0 & 48.4 & 45.7 & 602 & 0.58 & 1.08 & 8.5 & 12.0 & 50 \\
\hline 55 & 30.2 & 10.6 & 313 & 56.8 & 48.1 & 49.5 & 46.8 & 640 & 0.47 & 1.03 & 7.0 & 11.6 & 55 \\
\hline 60 & 30.7 & 10.4 & 313 & 59.0 & 49.0 & 50.4 & 47.6 & 672 & 0.38 & 0.98 & 5.7 & 11.2 & 60 \\
\hline
\end{tabular}




\begin{tabular}{|c|c|c|c|c|c|c|c|c|c|c|c|c|c|}
\hline \multirow{2}{*}{\multicolumn{3}{|c|}{$\begin{array}{l}\text { Populier, Nederland } 2018 \\
\text { Poplar, Netherlands } 2018\end{array}$}} & \multicolumn{8}{|c|}{ lijnbeplanting met plantafstand $3.2 \mathrm{~m}$, zonder dunning } & \multirow{2}{*}{\multicolumn{3}{|c|}{$\begin{array}{r}\text { Boniteit IV, } \boldsymbol{h}_{\mathbf{2 5}}=\mathbf{1 9 . 7} \\
\text { Site Class IV, } h_{25}=19.7 \\
\end{array}$}} \\
\hline & & & & & ntatio & th spa & $3.2 \mathrm{~m}$, & out $\mathrm{t}$ & & & & & \\
\hline \multirow[b]{2}{*}{$t$} & \multicolumn{8}{|c|}{$\begin{array}{l}\text { Kenmerken blijvende opstand } \\
\text { Characteristics remaining stand }\end{array}$} & \multicolumn{4}{|c|}{$\begin{array}{l}\text { Bijgroei } \\
\text { Increment }\end{array}$} & \multirow[b]{2}{*}{$t$} \\
\hline & $h_{m}$ & S\% & $N$ & $\boldsymbol{G}$ & $d_{g}$ & $d_{o r}$ & $d_{i r}$ & $v$ & $I c_{G}$ & $I m_{G}$ & $\| c_{V}$ & $I m_{V}$ & \\
\hline 5 & 5.1 & 63.0 & 313 & 1.7 & 8.3 & 8.5 & 8.1 & 4 & 1.07 & 0.34 & 3.2 & 0.8 & 5 \\
\hline 10 & 10.2 & 31.4 & 313 & 5.6 & 15.1 & 15.5 & 14.6 & 27 & 1.02 & 0.56 & 6.8 & 2.7 & 10 \\
\hline 15 & 14.2 & 22.6 & 313 & 11.0 & 21.2 & 21.9 & 20.5 & 69 & 1.11 & 0.73 & 9.6 & 4.6 & 15 \\
\hline 20 & 17.3 & 18.5 & 313 & 16.5 & 25.9 & 26.8 & 25.1 & 121 & 1.07 & 0.82 & 10.8 & 6.0 & 20 \\
\hline 25 & 19.7 & 16.2 & 313 & 21.6 & 29.7 & 30.7 & 28.7 & 175 & 0.97 & 0.86 & 10.7 & 7.0 & 25 \\
\hline 30 & 21.6 & 14.8 & 313 & 26.1 & 32.6 & 33.8 & 31.5 & 227 & 0.84 & 0.87 & 10.0 & 7.6 & 30 \\
\hline 35 & 23.0 & 13.9 & 313 & 30.0 & 35.0 & 36.2 & 33.8 & 274 & 0.72 & 0.86 & 8.9 & 7.8 & 35 \\
\hline 40 & 24.1 & 13.3 & 313 & 33.3 & 36.8 & 38.1 & 35.6 & 316 & 0.60 & 0.83 & 7.7 & 7.9 & 40 \\
\hline 45 & 25.0 & 12.8 & 313 & 36.1 & 38.3 & 39.7 & 37.0 & 351 & 0.50 & 0.80 & 6.5 & 7.8 & 45 \\
\hline 50 & 25.6 & 12.5 & 313 & 38.3 & 39.5 & 40.9 & 38.2 & 380 & 0.41 & 0.77 & 5.4 & 7.6 & 50 \\
\hline 55 & 26.1 & 12.2 & 313 & 40.2 & 40.5 & 41.9 & 39.1 & 405 & 0.33 & 0.73 & 4.4 & 7.4 & 55 \\
\hline 60 & 26.5 & 12.1 & 313 & 41.7 & 41.2 & 42.7 & 39.8 & 425 & 0.27 & 0.69 & 3.6 & 7.1 & 60 \\
\hline
\end{tabular}




\begin{tabular}{|c|c|c|c|c|c|c|c|c|c|c|c|c|c|}
\hline \multirow{2}{*}{\multicolumn{3}{|c|}{$\begin{array}{l}\text { Populier, Nederland } 2018 \\
\text { Poplar, Netherlands } 2018\end{array}$}} & \multirow{2}{*}{\multicolumn{8}{|c|}{ lijnbeplanting met plantafstand $3.2 \mathrm{~m}$, zonder dunning }} & \multirow{2}{*}{\multicolumn{3}{|c|}{$\begin{array}{r}\text { Boniteit V, } \boldsymbol{h}_{25}=\mathbf{1 6 . 7} \\
\text { Site Class } V, h_{25}=16.7\end{array}$}} \\
\hline & & & & line & & & $3.2 \mathrm{~m}$ & out $\mathrm{t}$ & & & & & \\
\hline \multirow[b]{2}{*}{$t$} & \multicolumn{8}{|c|}{$\begin{array}{l}\text { Kenmerken blijvende opstand } \\
\text { Characteristics remaining stand }\end{array}$} & \multicolumn{4}{|c|}{$\begin{array}{l}\text { Bijgroei } \\
\text { Increment }\end{array}$} & \multirow[b]{2}{*}{$t$} \\
\hline & $\boldsymbol{h}_{m}$ & $S \%$ & $N$ & $G$ & $d_{g}$ & $d_{o r}$ & $d_{i r}$ & $v$ & $I c_{G}$ & $I m_{G}$ & $I c_{v}$ & $I m_{V}$ & \\
\hline 5 & 4.5 & 71.3 & 313 & 1.2 & 6.9 & 7.1 & 6.7 & 2 & 0.77 & 0.23 & 2.0 & 0.5 & 5 \\
\hline 10 & 8.7 & 36.6 & 313 & 3.8 & 12.4 & 12.9 & 12.0 & 17 & 0.70 & 0.38 & 4.1 & 1.7 & 10 \\
\hline 15 & 12.1 & 26.5 & 313 & 7.5 & 17.5 & 18.2 & 16.8 & 42 & 0.76 & 0.50 & 5.8 & 2.8 & 15 \\
\hline 20 & 14.7 & 21.8 & 313 & 11.2 & 21.4 & 22.3 & 20.6 & 73 & 0.73 & 0.56 & 6.5 & 3.6 & 20 \\
\hline 25 & 16.7 & 19.2 & 313 & 14.7 & 24.5 & 25.5 & 23.5 & 105 & 0.66 & 0.59 & 6.4 & 4.2 & 25 \\
\hline 30 & 18.3 & 17.5 & 313 & 17.8 & 26.9 & 28.1 & 25.9 & 136 & 0.58 & 0.59 & 6.0 & 4.5 & 30 \\
\hline 35 & 19.5 & 16.4 & 313 & 20.5 & 28.9 & 30.1 & 27.7 & 165 & 0.49 & 0.59 & 5.3 & 4.7 & 35 \\
\hline 40 & 20.4 & 15.7 & 313 & 22.7 & 30.4 & 31.7 & 29.2 & 189 & 0.41 & 0.57 & 4.6 & 4.7 & 40 \\
\hline 45 & 21.1 & 15.2 & 313 & 24.6 & 31.7 & 33.0 & 30.4 & 210 & 0.34 & 0.55 & 3.9 & 4.7 & 45 \\
\hline 50 & 21.7 & 14.8 & 313 & 26.1 & 32.6 & 34.0 & 31.3 & 228 & 0.28 & 0.52 & 3.2 & 4.6 & 50 \\
\hline 55 & 22.1 & 14.5 & 313 & 27.4 & 33.4 & 34.8 & 32.1 & 243 & 0.23 & 0.50 & 2.6 & 4.4 & 55 \\
\hline 60 & 22.4 & 14.3 & 313 & 28.4 & 34.0 & 35.5 & 32.7 & 255 & 0.19 & 0.47 & 2.2 & 4.2 & 60 \\
\hline
\end{tabular}




\section{Trilpopulier (Populus tremula)}

Aspen

Jansen, J.J., G.M.J. Mohren, P. Schmidt, L. Goudzwaard, A. Oosterbaan en J. den Ouden

Bron: Jansen, J.J., G.M.J. Mohren, P. Schmidt, L. Goudzwaard, A. Oosterbaan en J. den Ouden, 2018. Groei en productie van populier in Nederland. FEM Groei en Productie Rapport 2018 - 8, 127 blz.

Dit rapport is gratis te downloaden op: https://doi.org/10.18174/444097

Voor de trilpopulier is alleen een tabel met sterke dunning beschikbaar.

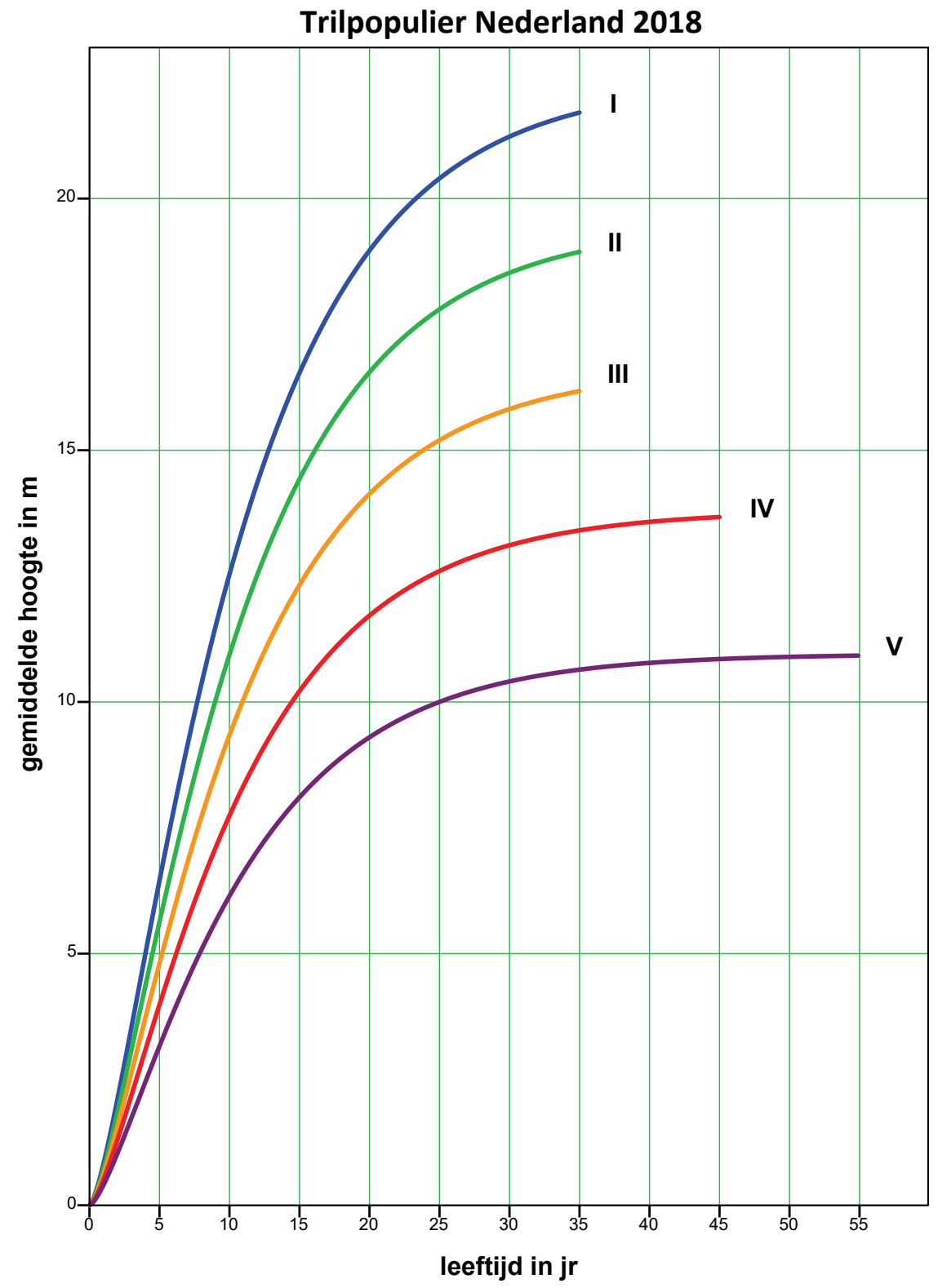




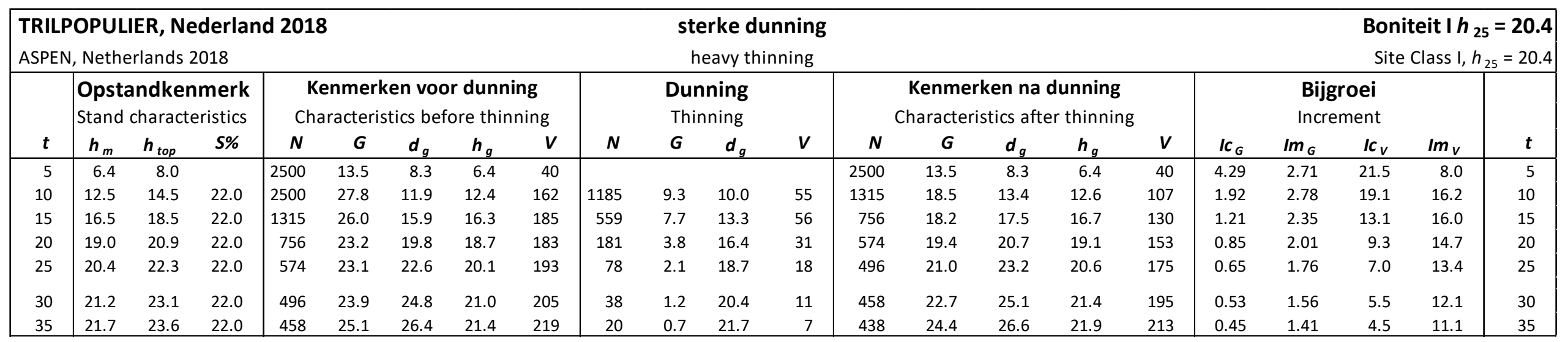

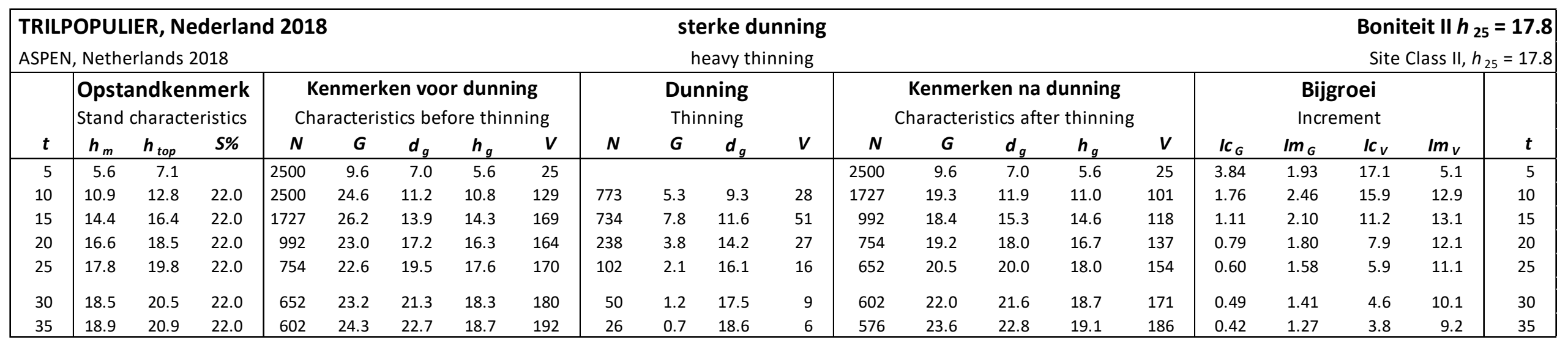




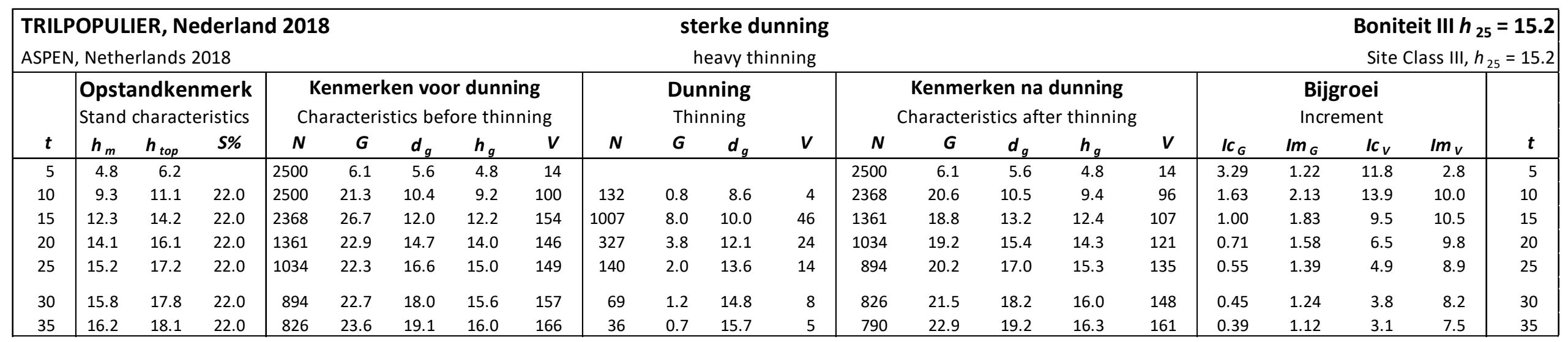

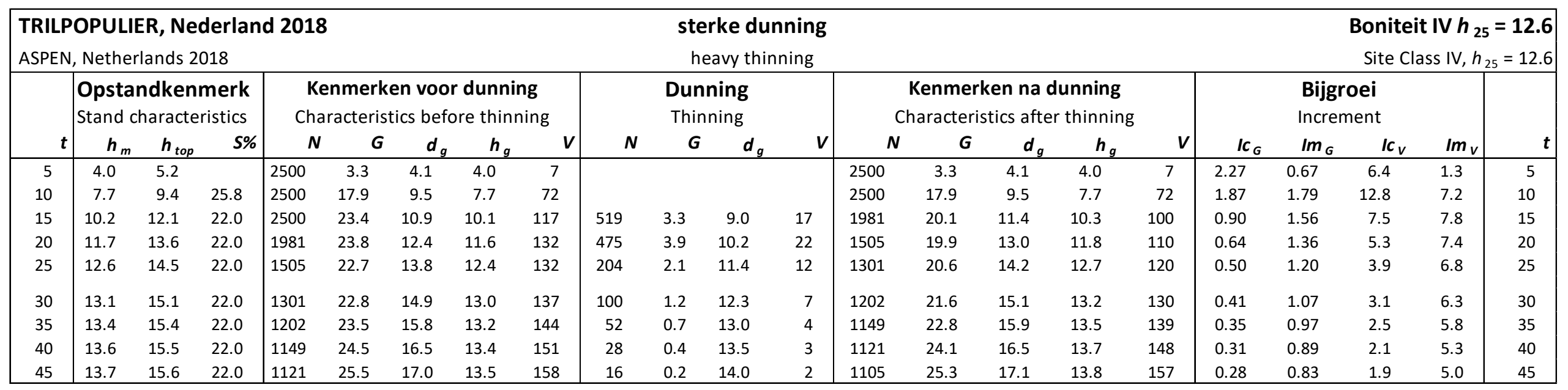




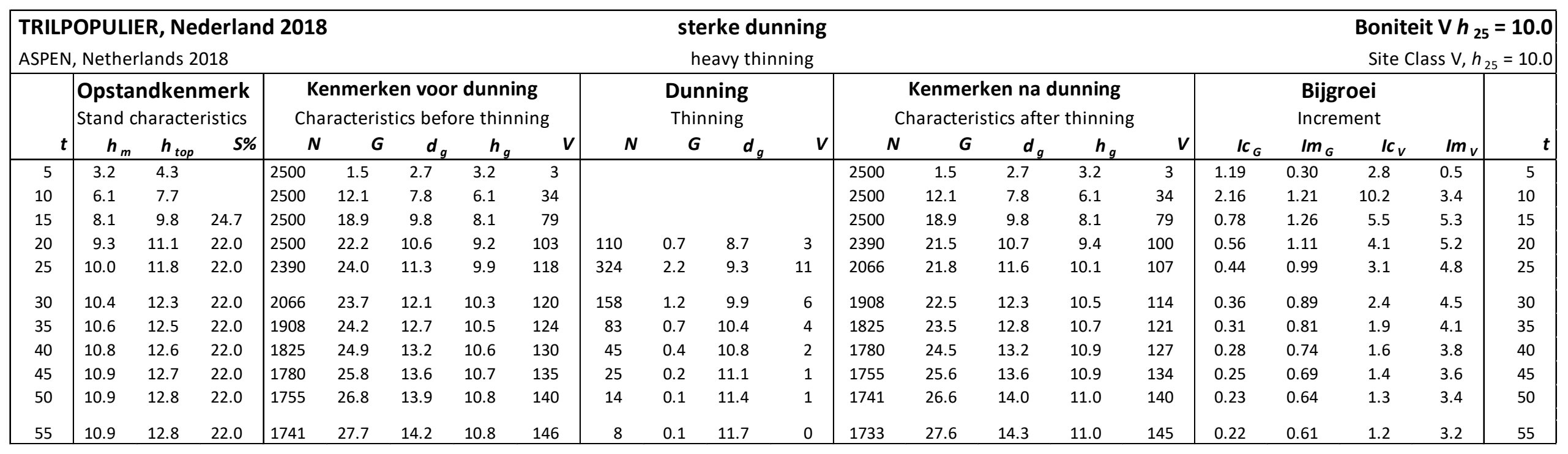




\section{Ruwe berk (Betula pendula)}

Jansen, J.J., A. Oosterbaan, G.M. Mohren en J. den Ouden

Bron: Jansen, J.J., A. Oosterbaan, G.M. Mohren en J. den Ouden, 2018. Groei en productie van ruwe berk in Nederland, FEM Groei en Productie Rapport 2018 - 13, 41 blz.

Dit rapport is gratis te downloaden op:

https://doi.org/10.18174/444102

Voor de ruwe berk is alleen een tabel met sterke dunning beschikbaar.

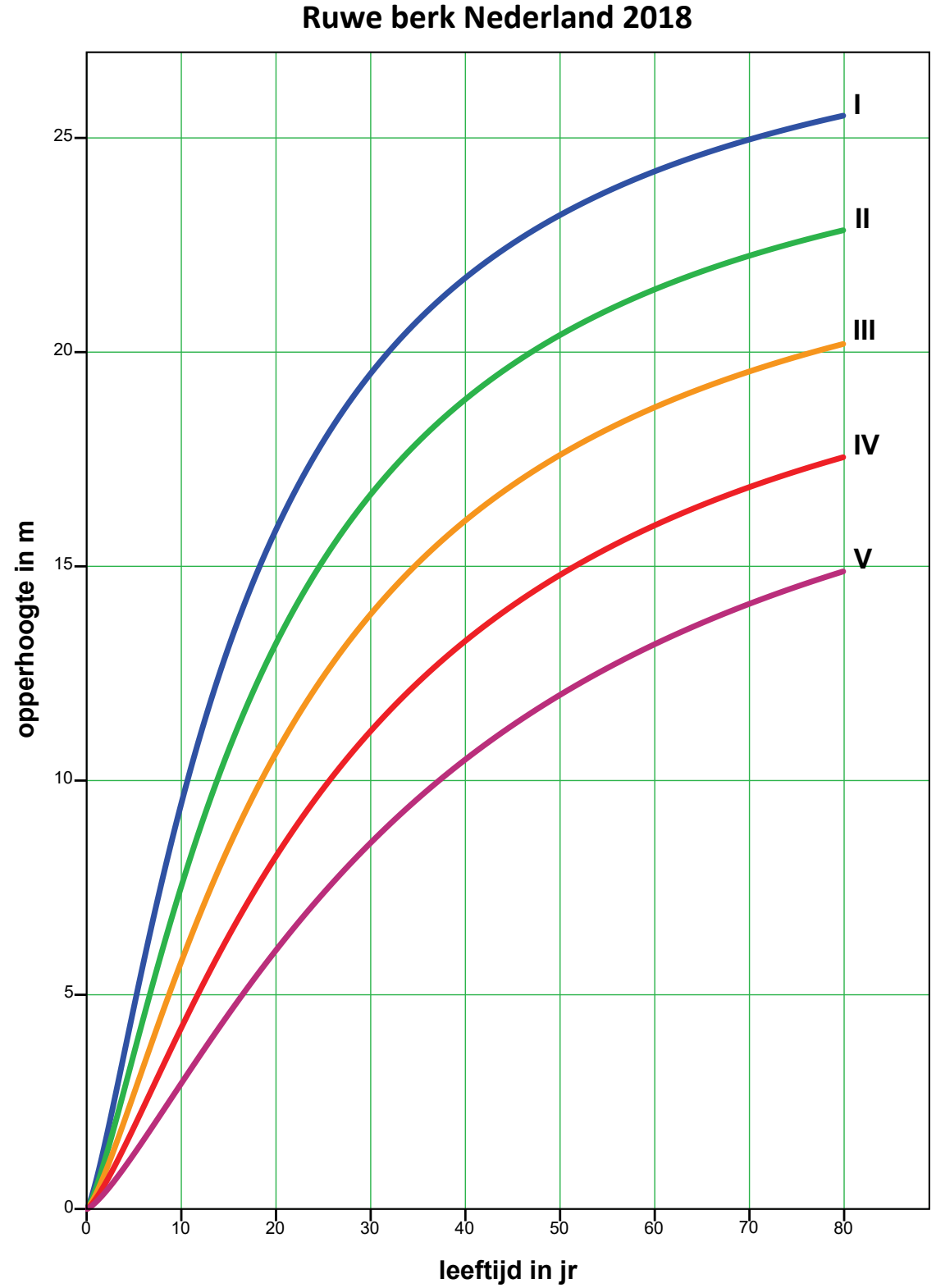




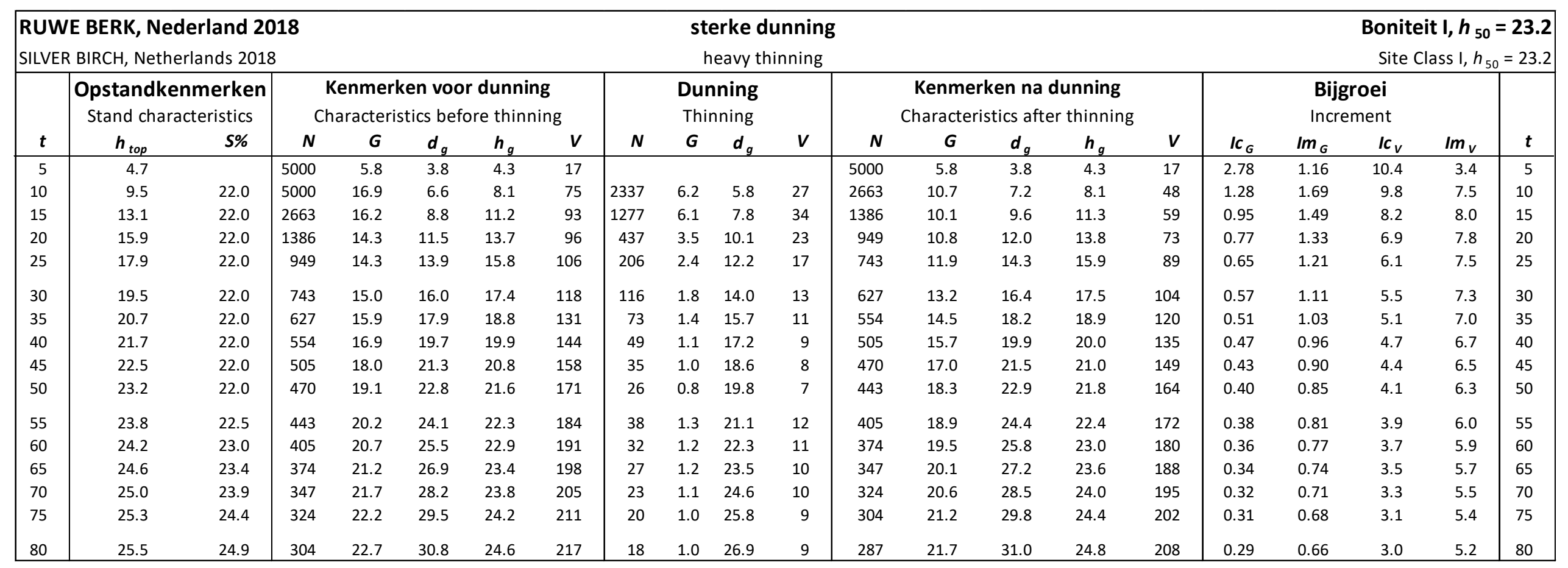




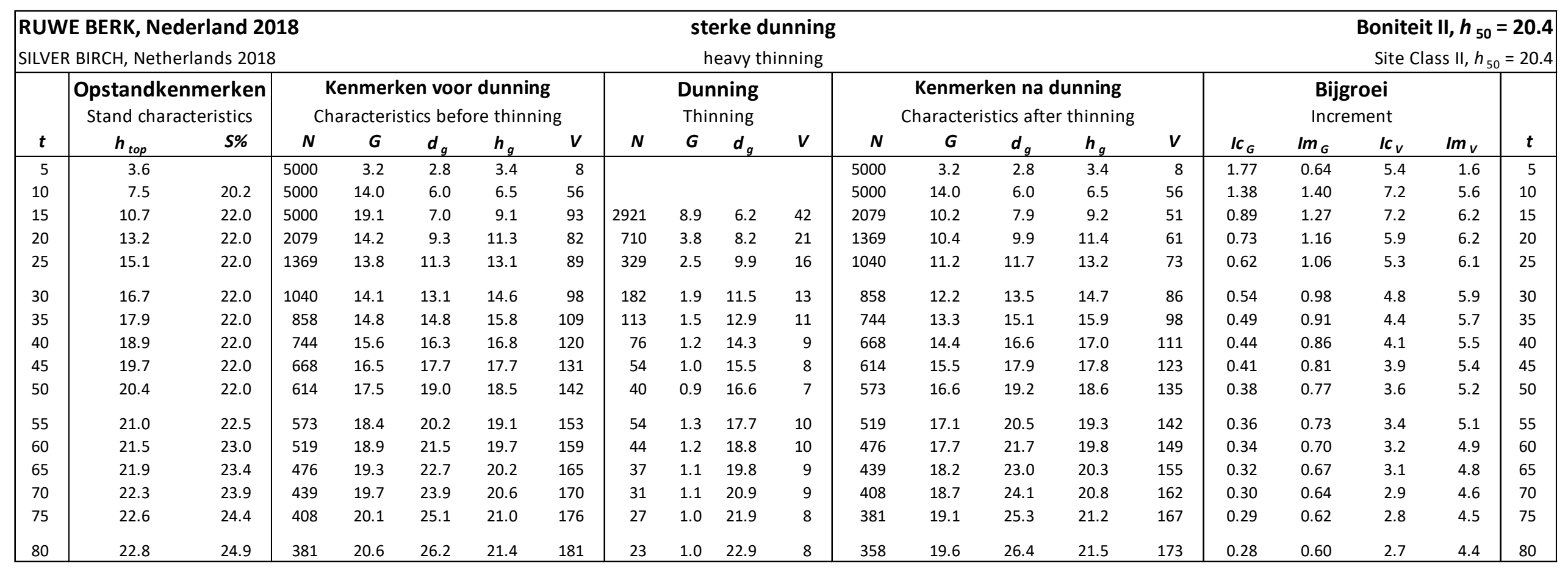




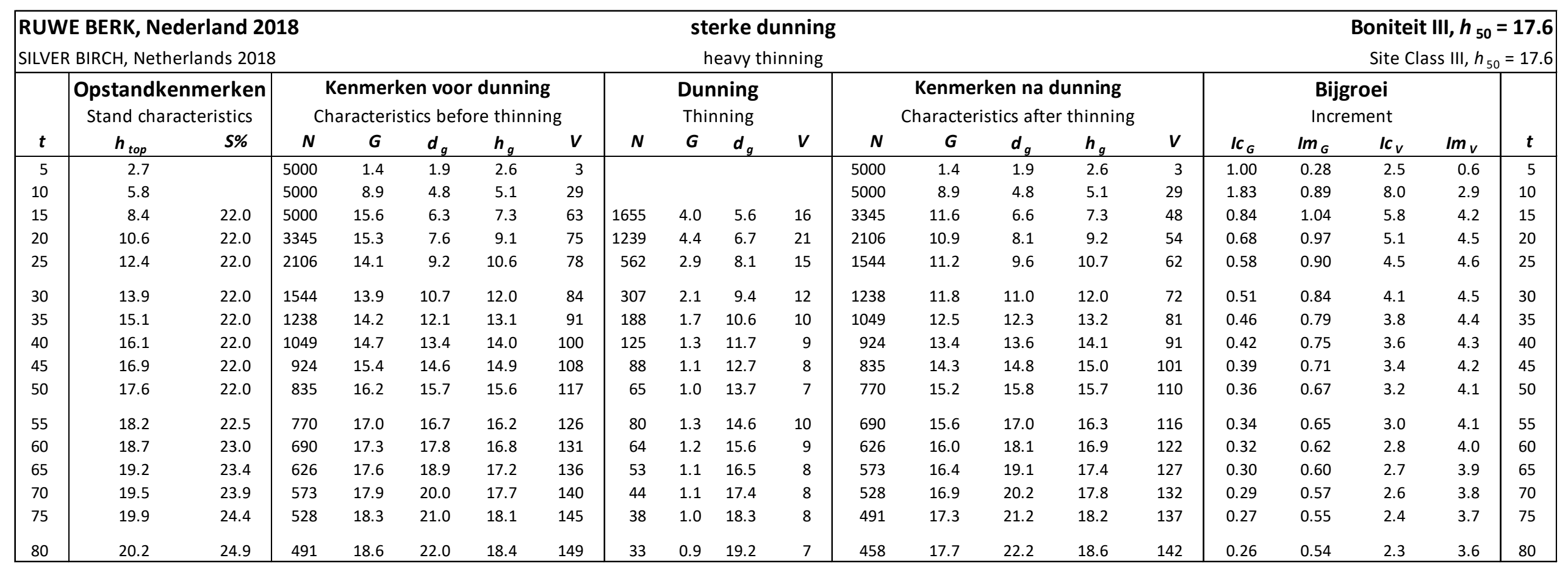




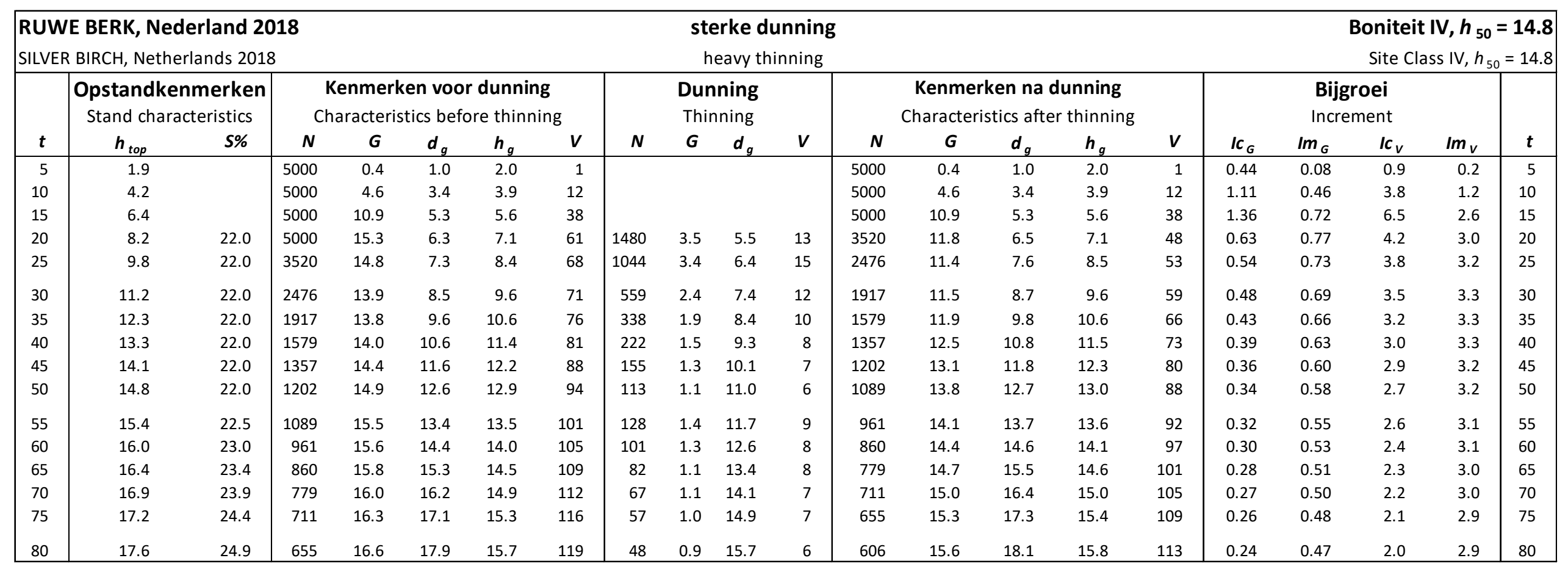




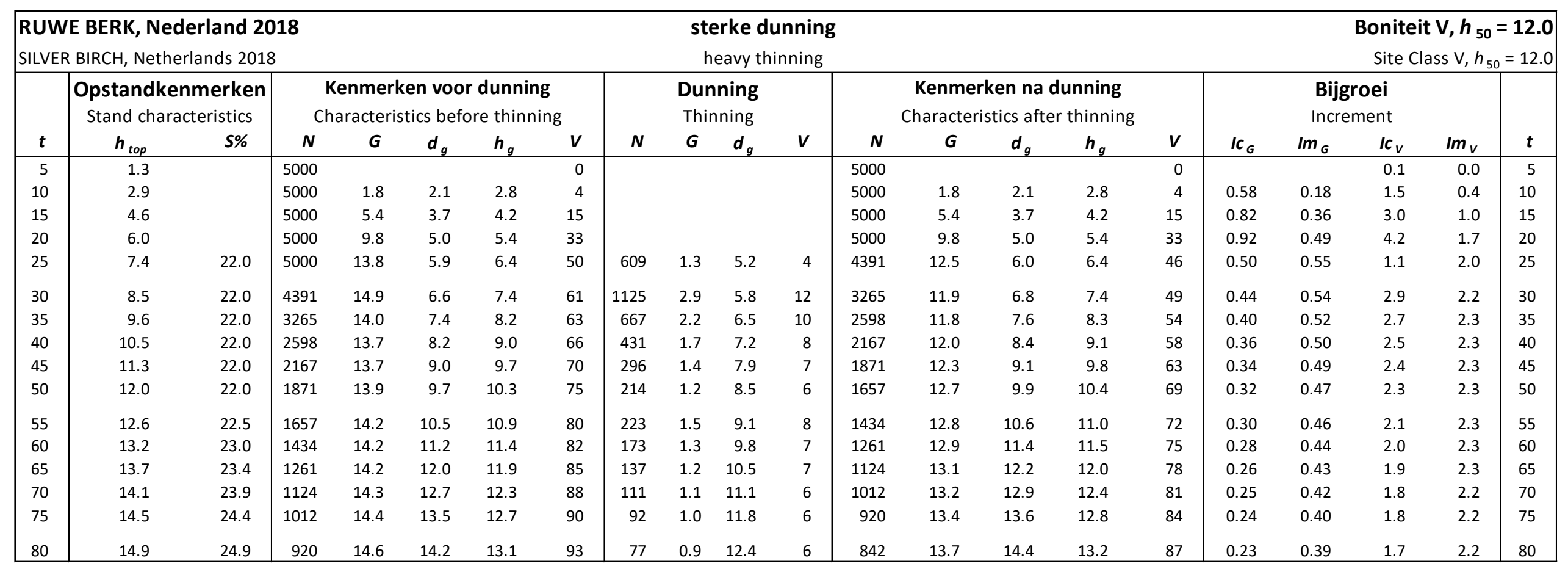




\section{Es (Fraxinus excelsior)}

Ash

Jansen, J.J. L. Goudzwaard, A. Oosterbaan, G.M.J. Mohren en J. den Ouden

Bron: Jansen, J.J. L. Goudzwaard, A. Oosterbaan, G.M.J. Mohren en J. den Ouden, 2018. Groei en productie van es in Nederland. FEM Groei en Productie Rapport 2018 - 11, 44 blz.

Dit rapport is gratis te downloaden op:

https://doi.org/10.18174/444100

Voor de es is alleen een tabel met matige dunning beschikbaar.

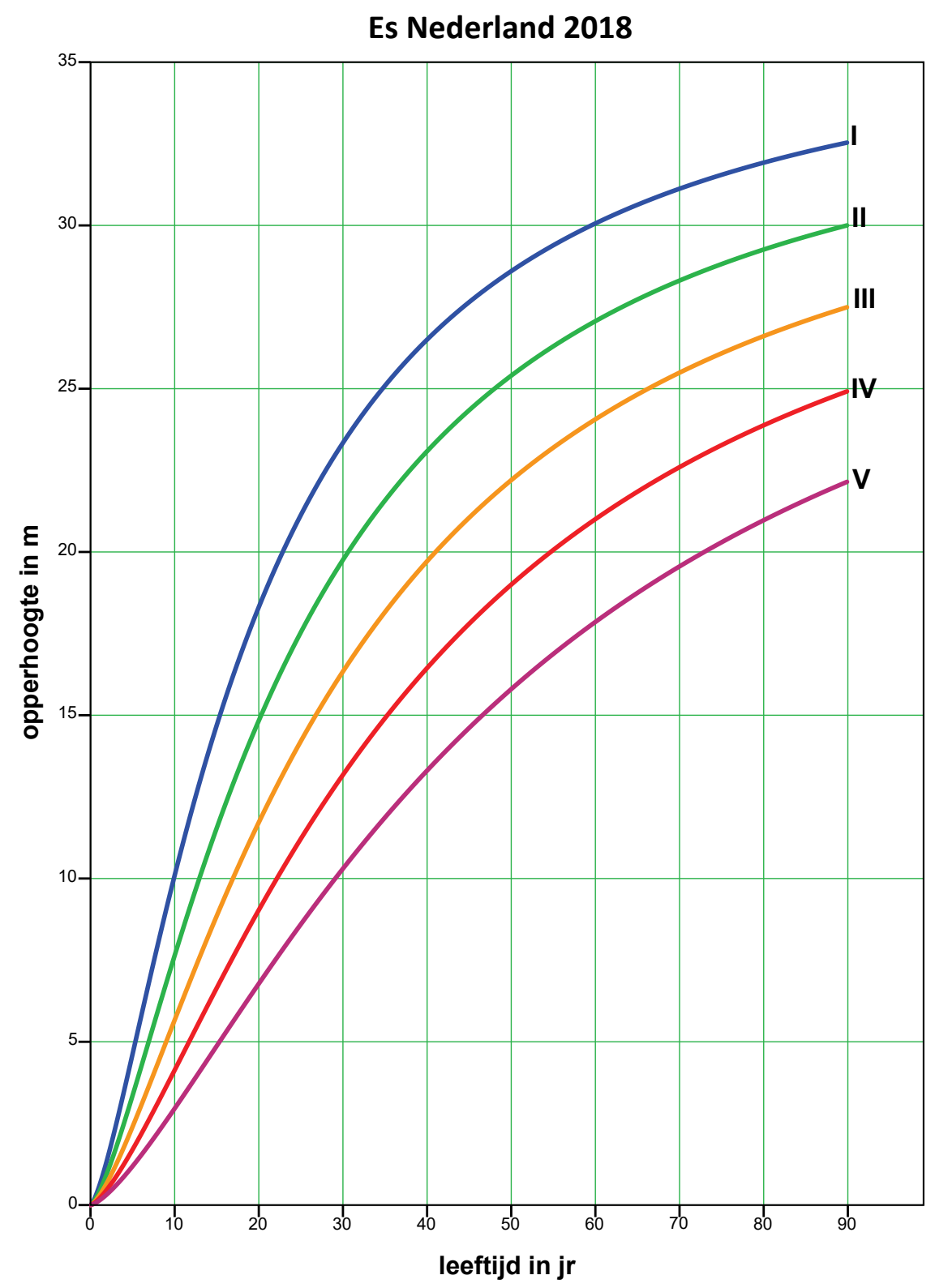




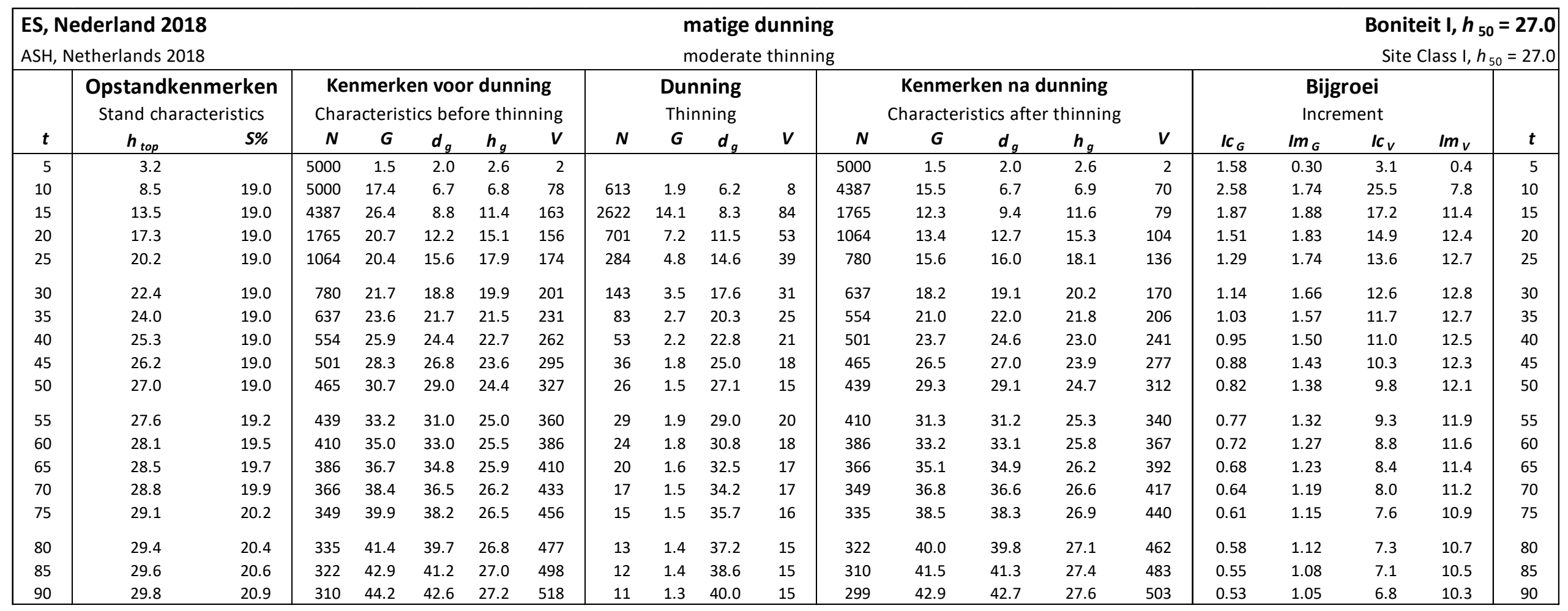




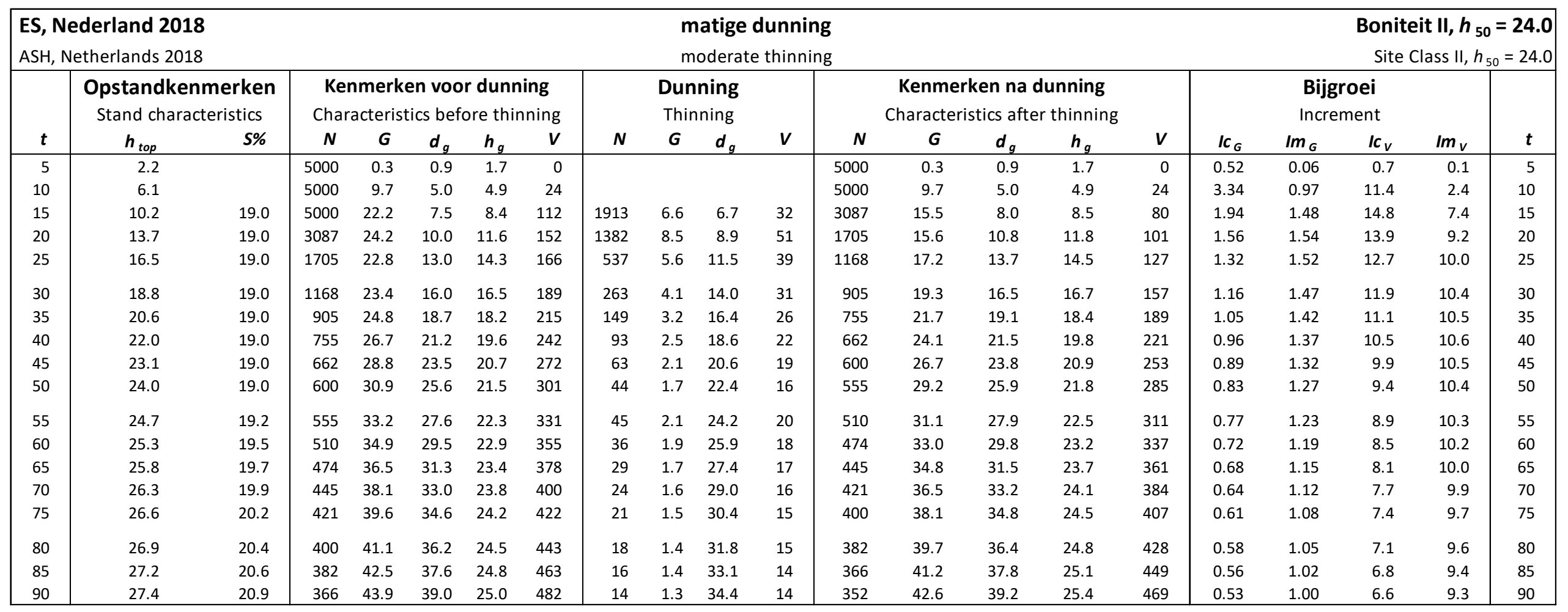




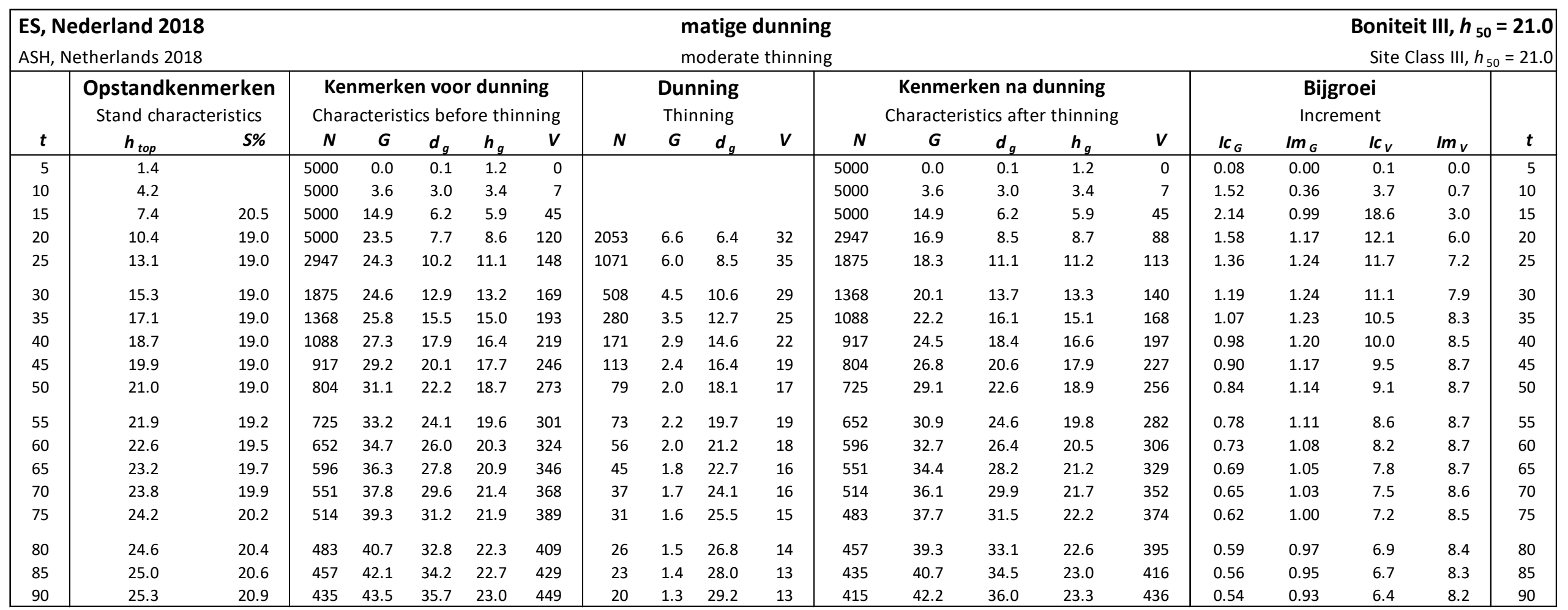




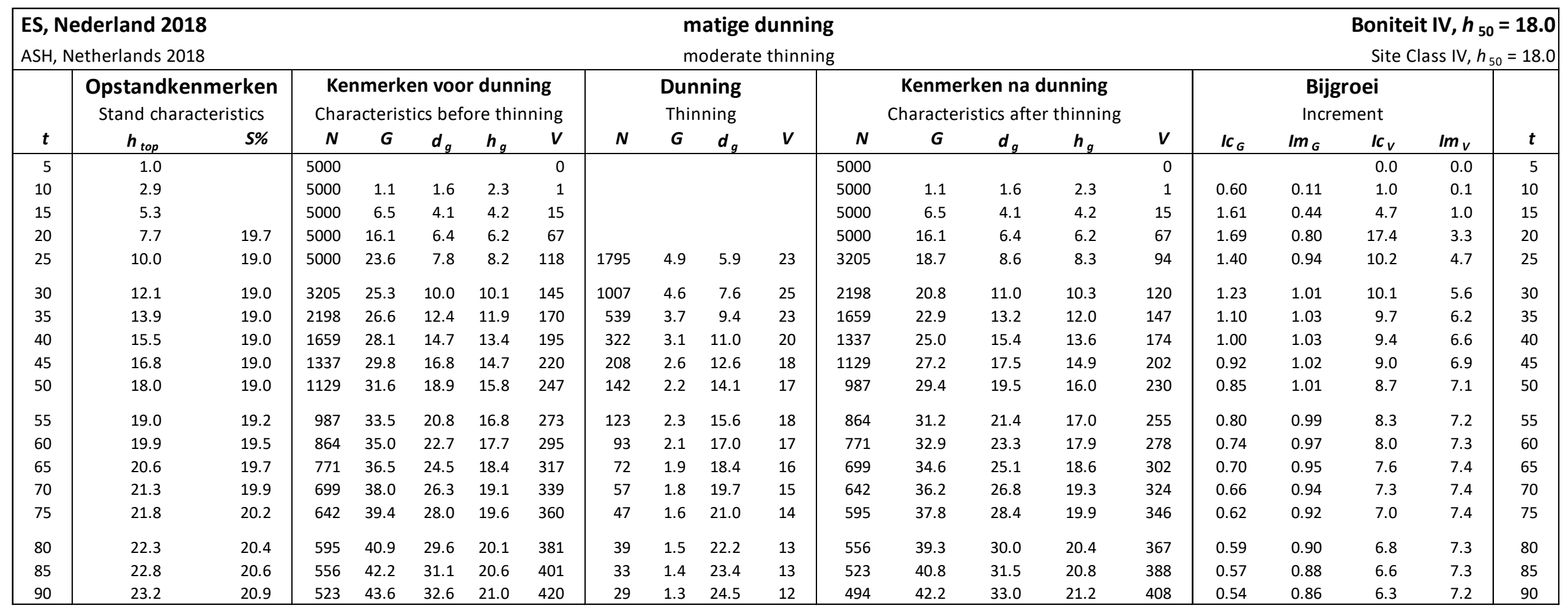




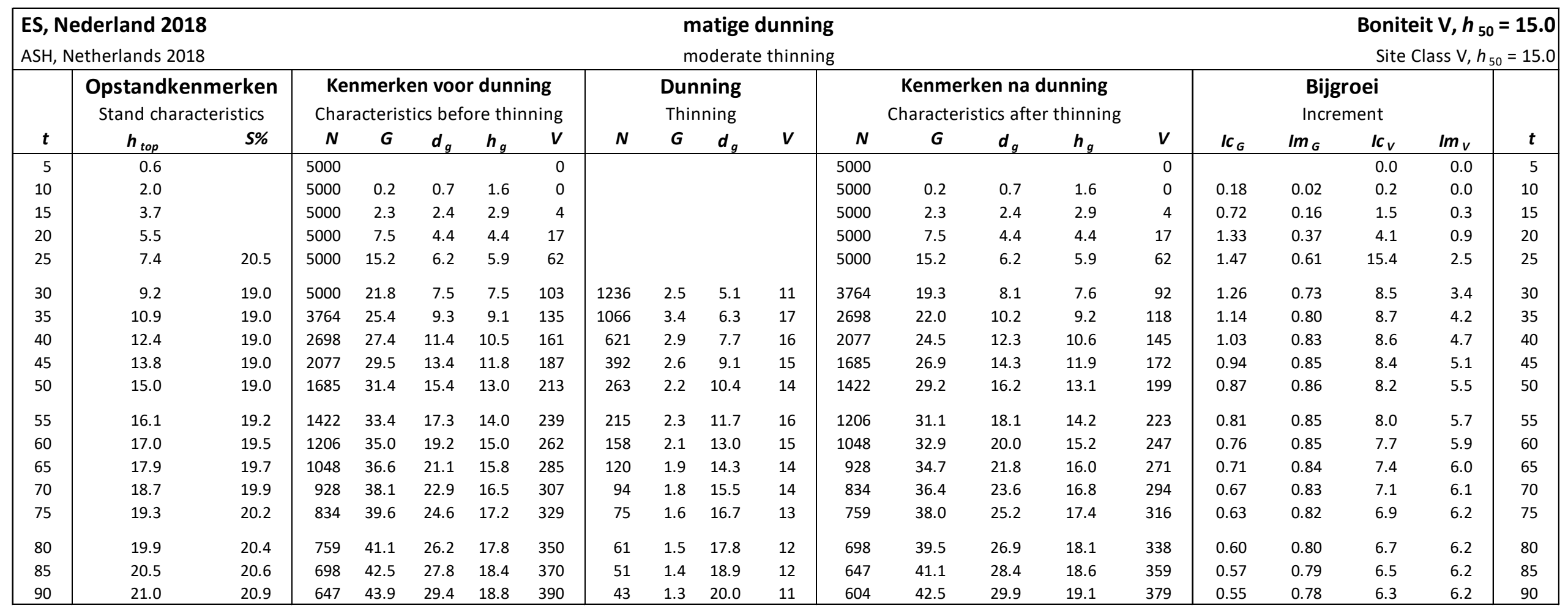




\section{Zwarte els (Alnus glutionosa)}

Bron: Jansen, J.J., A. Oosterbaan, G.M.J. Mohren, P. Copini en J. den Ouden, 2018. Groei en productie van zwarte els in Nederland. FEM Groei en Productie Rapport 2018 - 10, 47 blz.

Dit rapport is gratis te downloaden op:

https://doi.org/10.18174/444099

Voor de zwarte els is alleen een tabel met matige dunning beschikbaar

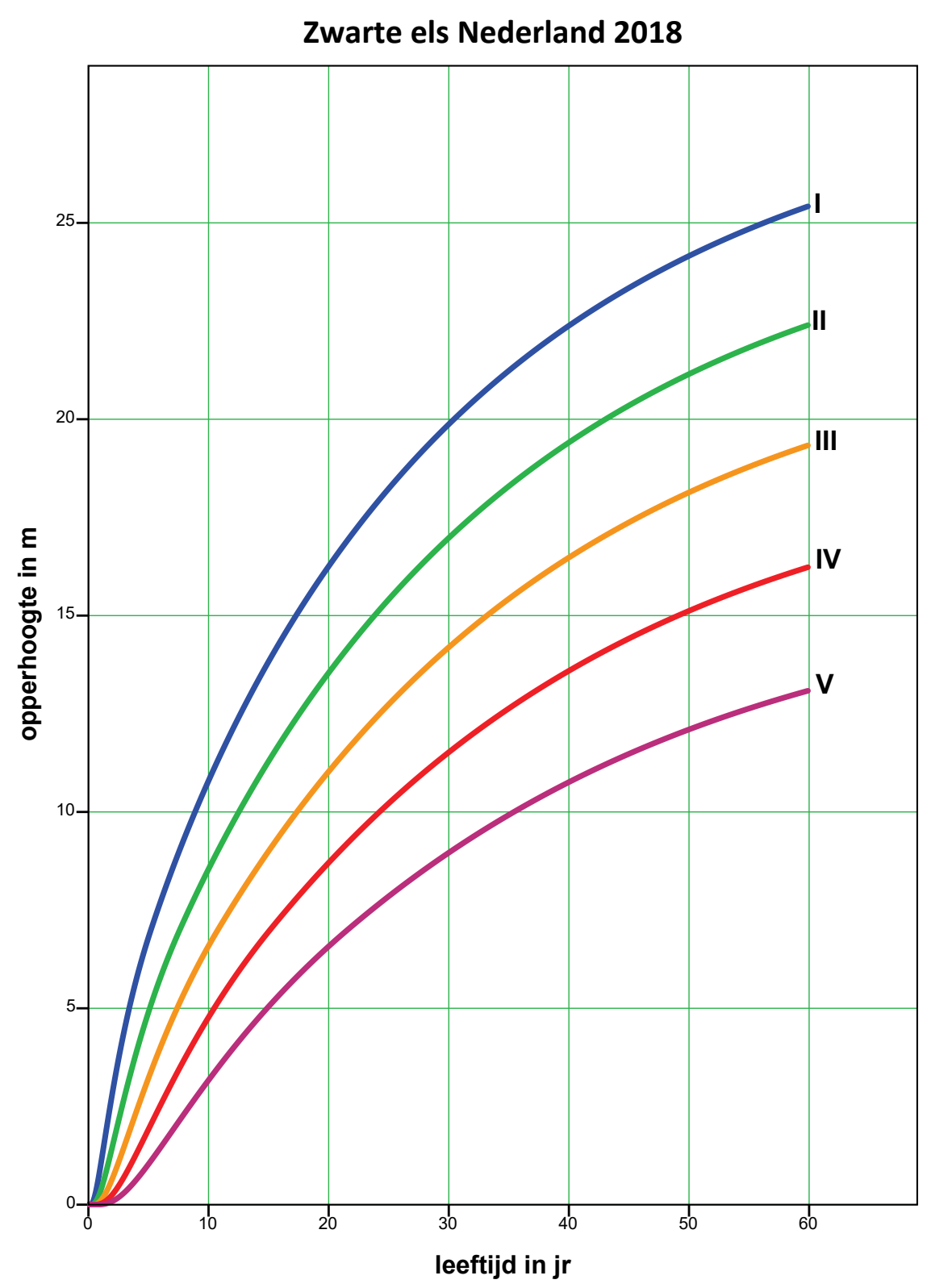




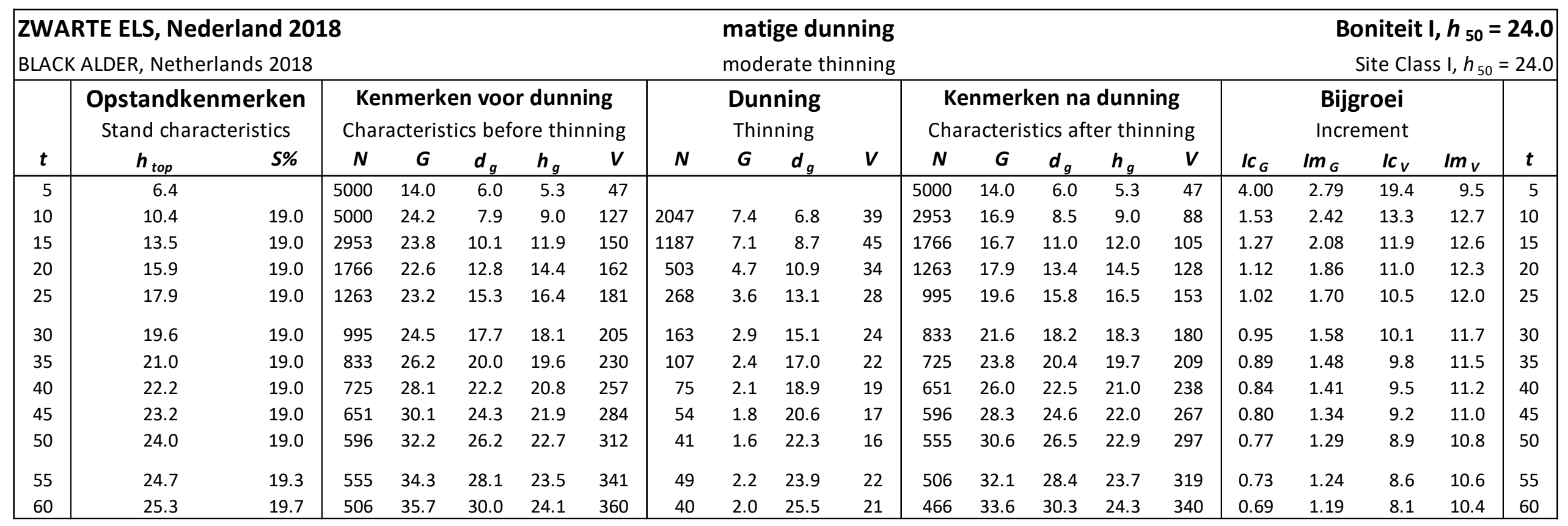




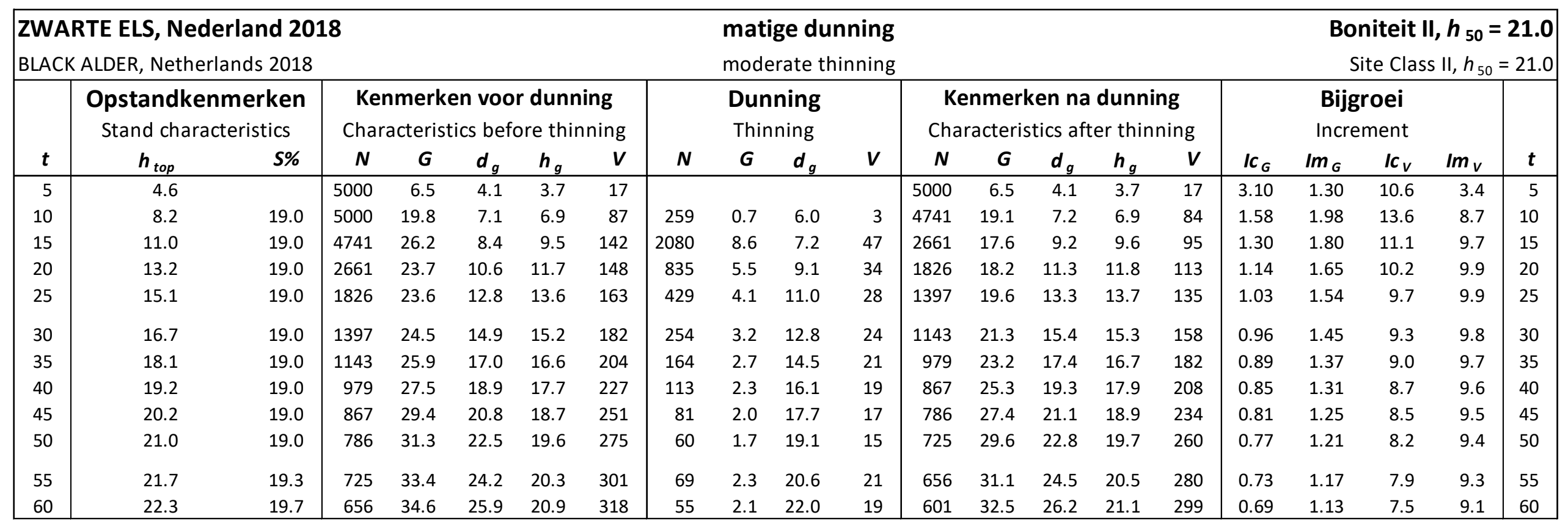




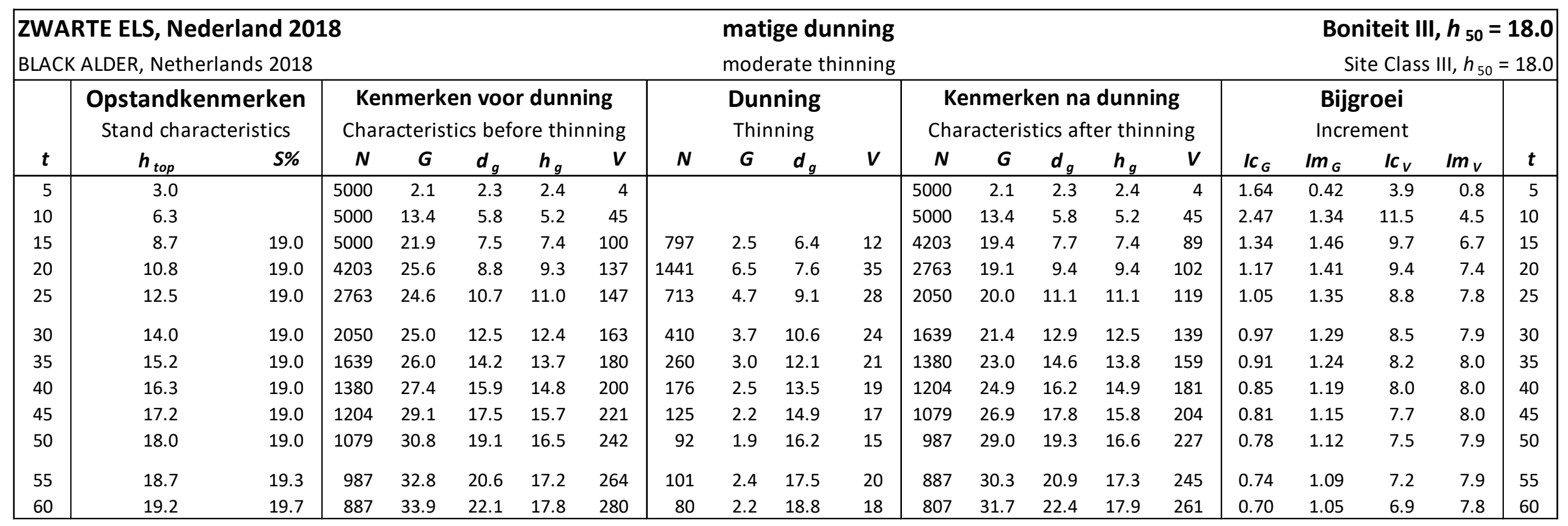




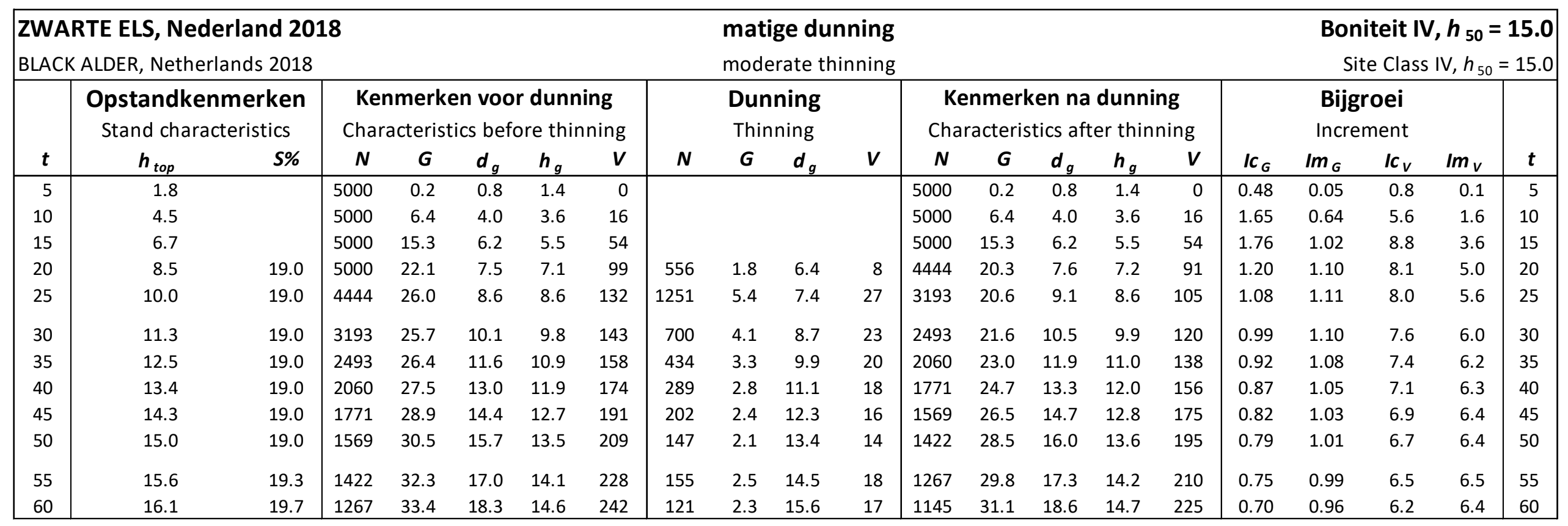




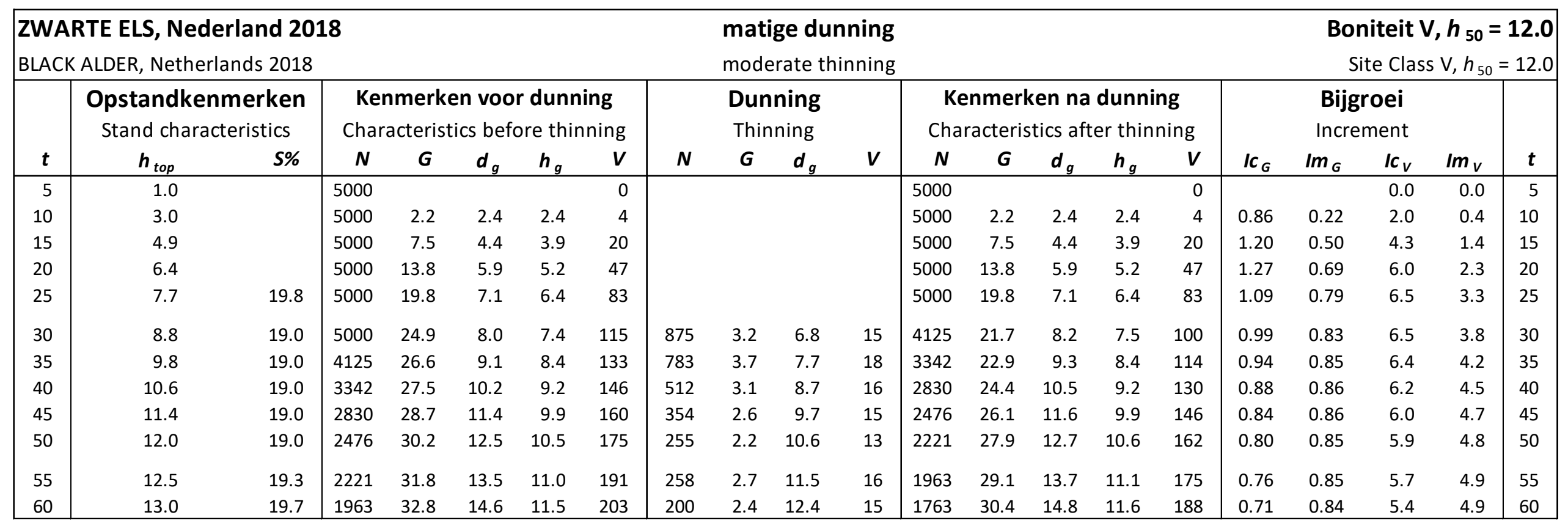




\section{Gewone Esdoorn (Acer pseudoplatanus)}

Jansen, J.J. A. Oosterbaan, G.M.J. Mohren en J. den Ouden

Bron: Jansen, J.J. A. Oosterbaan, G.M.J. Mohren en J. den Ouden, 2018. Groei en productie van gewone esdoorn in Nederland. FEM Groei en Productie Rapport 2018 - 12, 38 blz.

Dit rapport is gratis te downloaden op:

https://doi.org/10.18174/444101

Voor gewone esdoorn is alleen een tabel met sterke dunning beschikbaar.

\section{Sycamore}

.

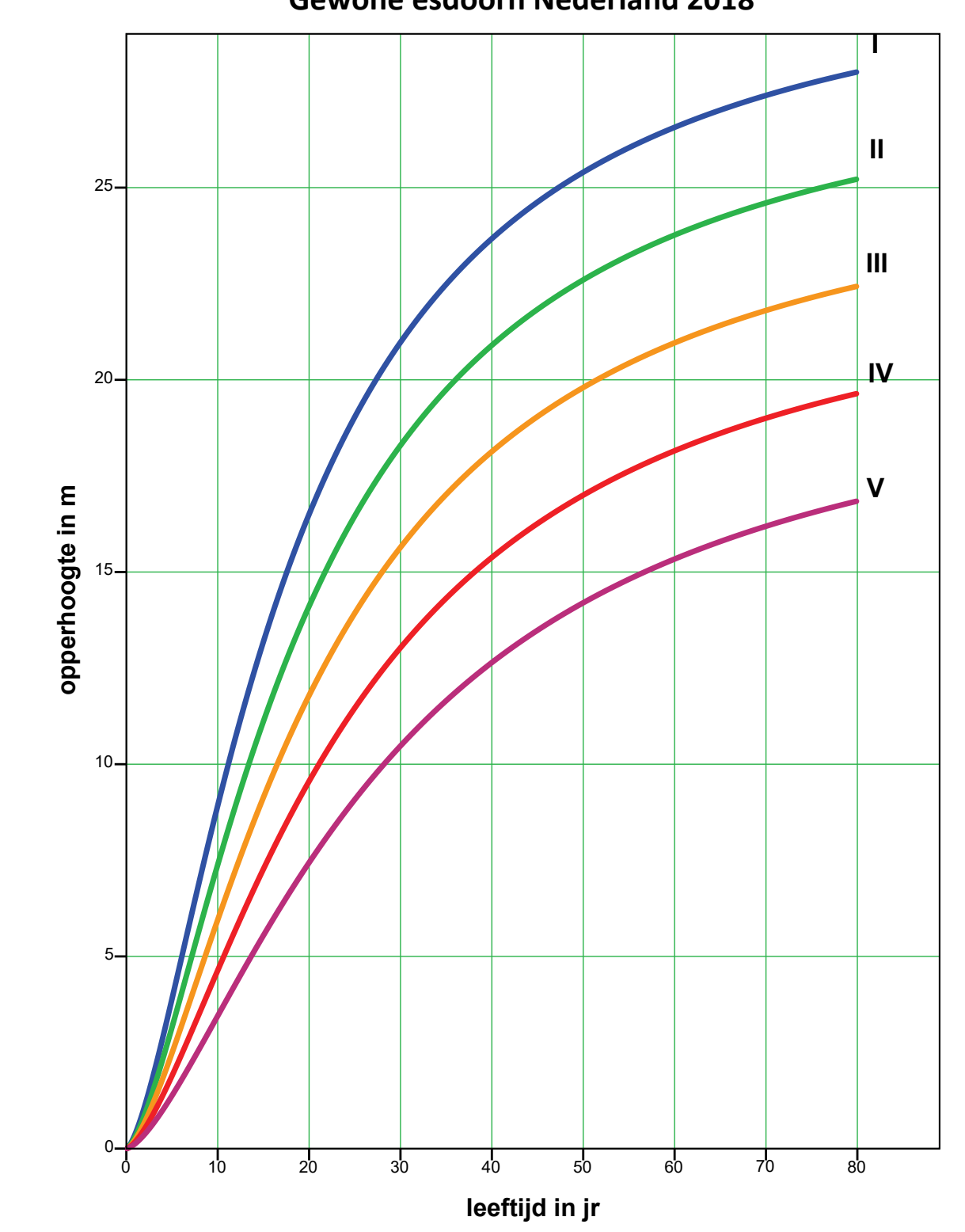




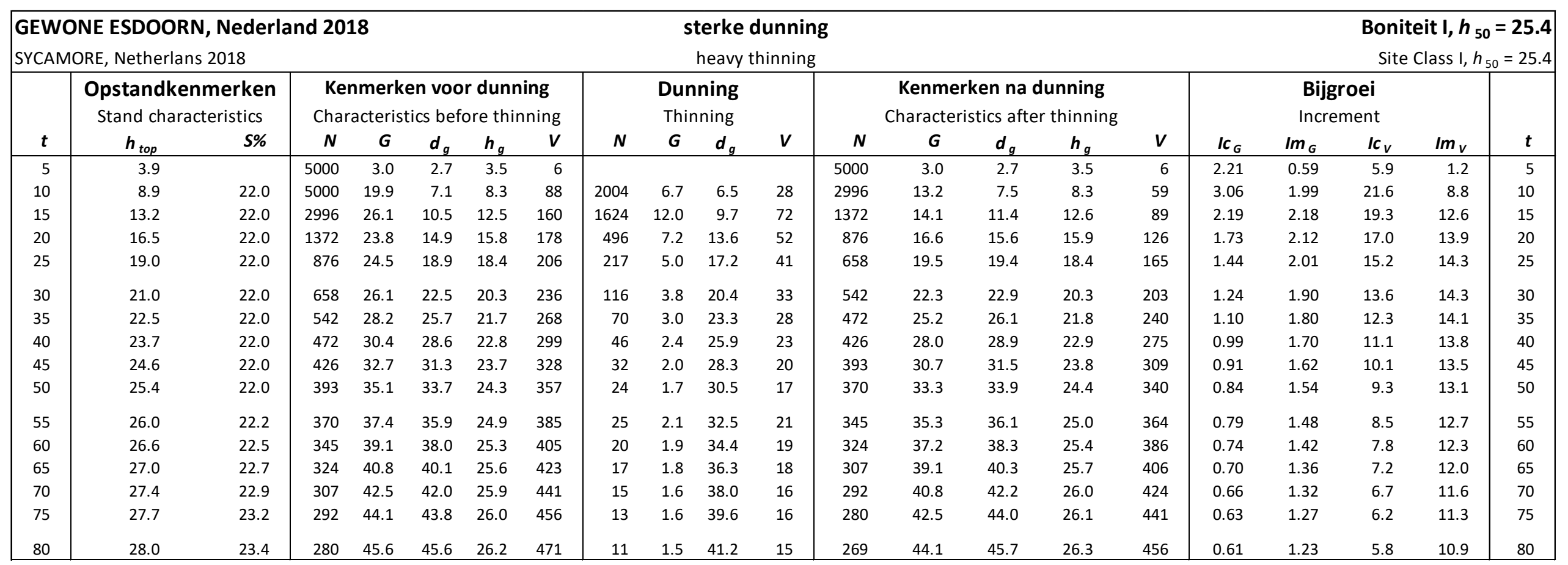




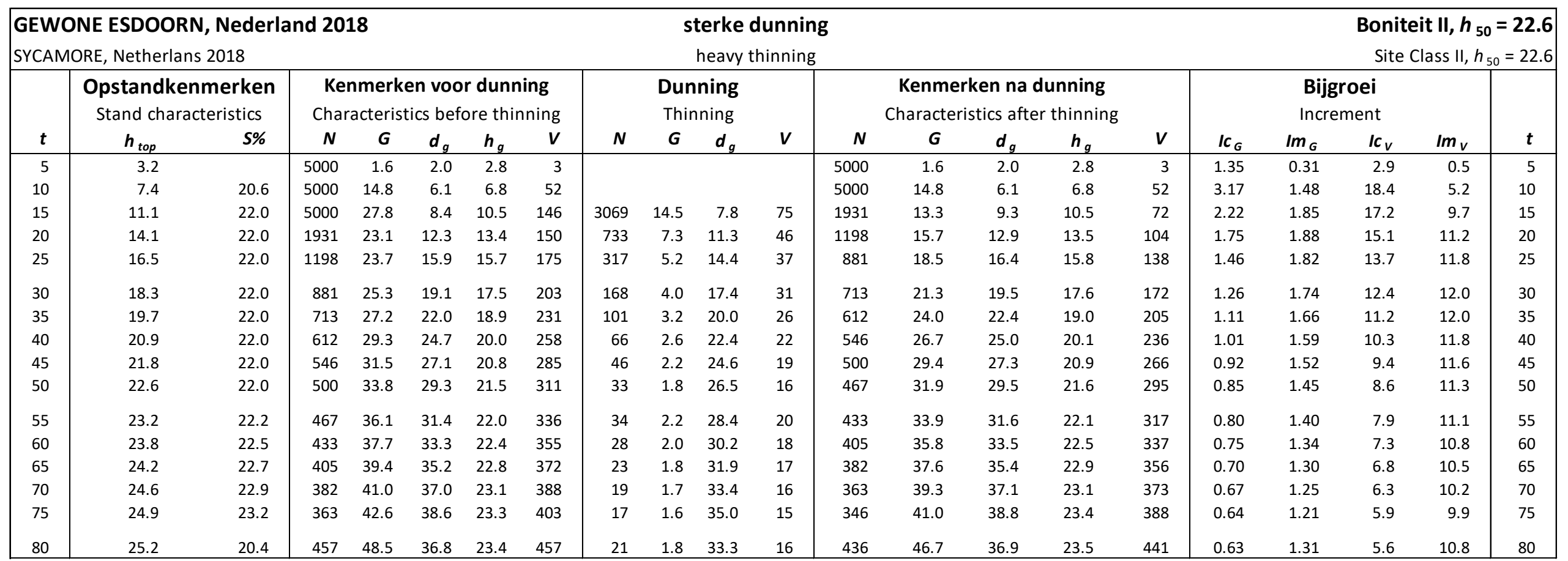




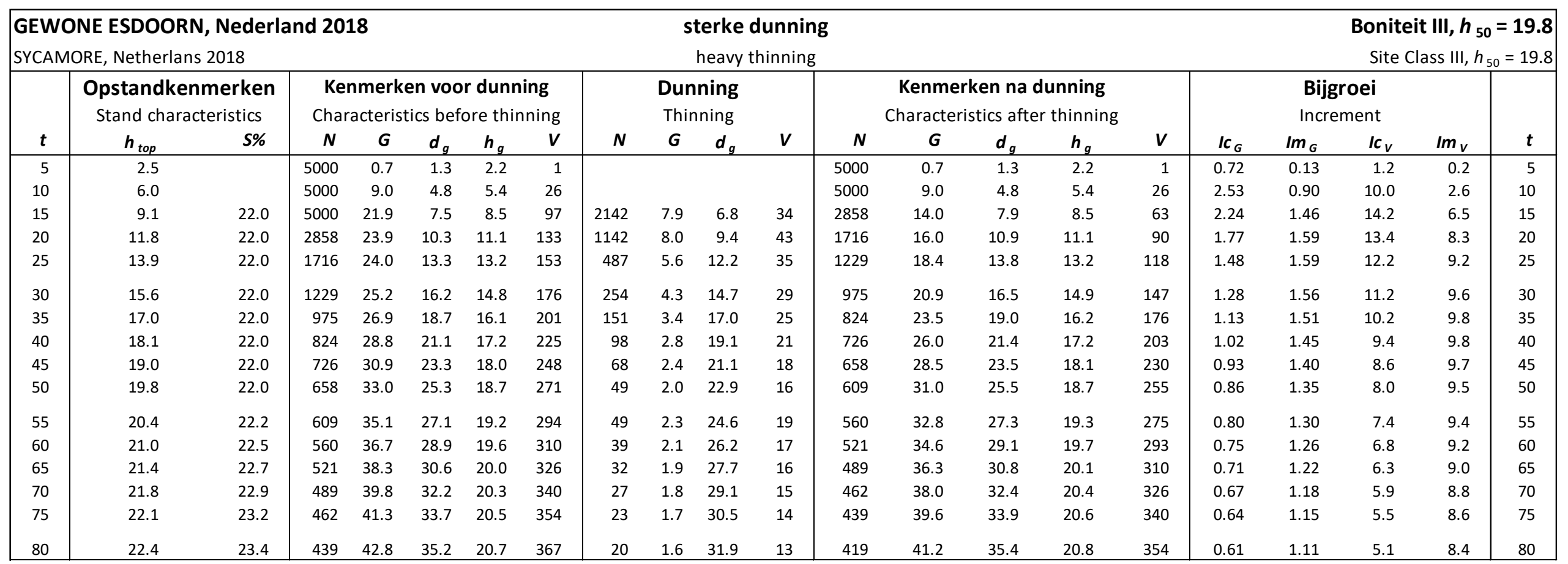




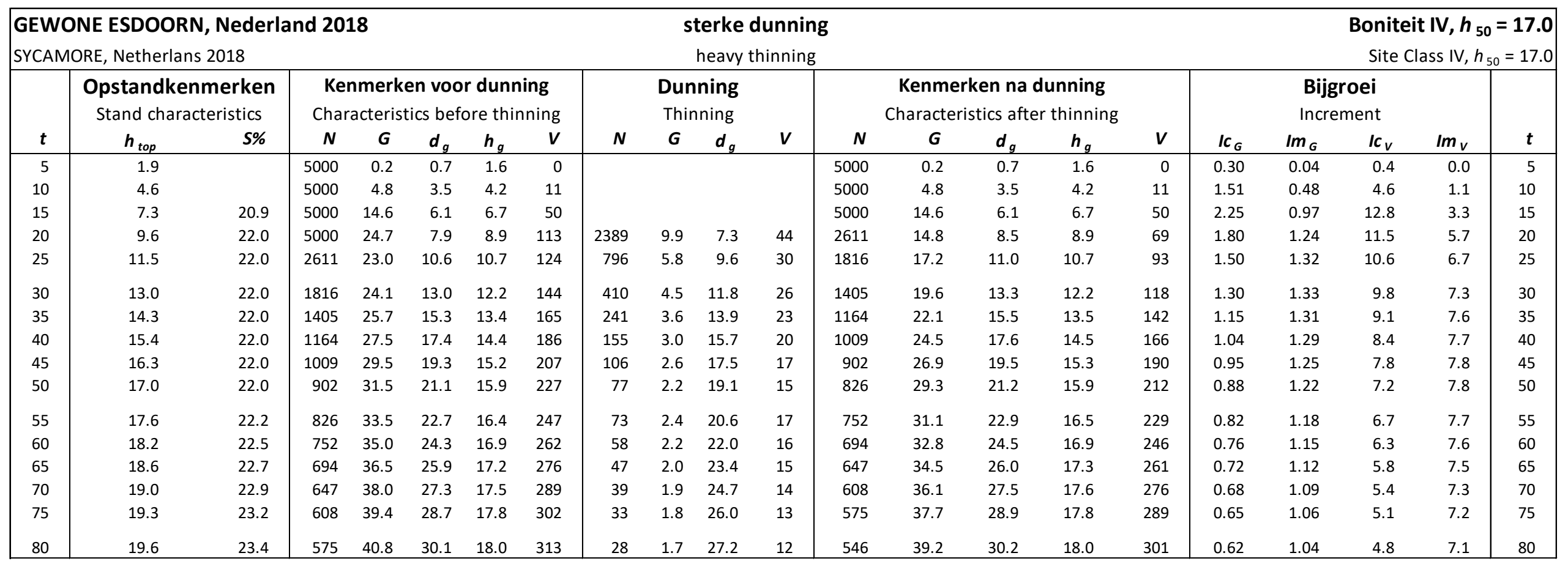




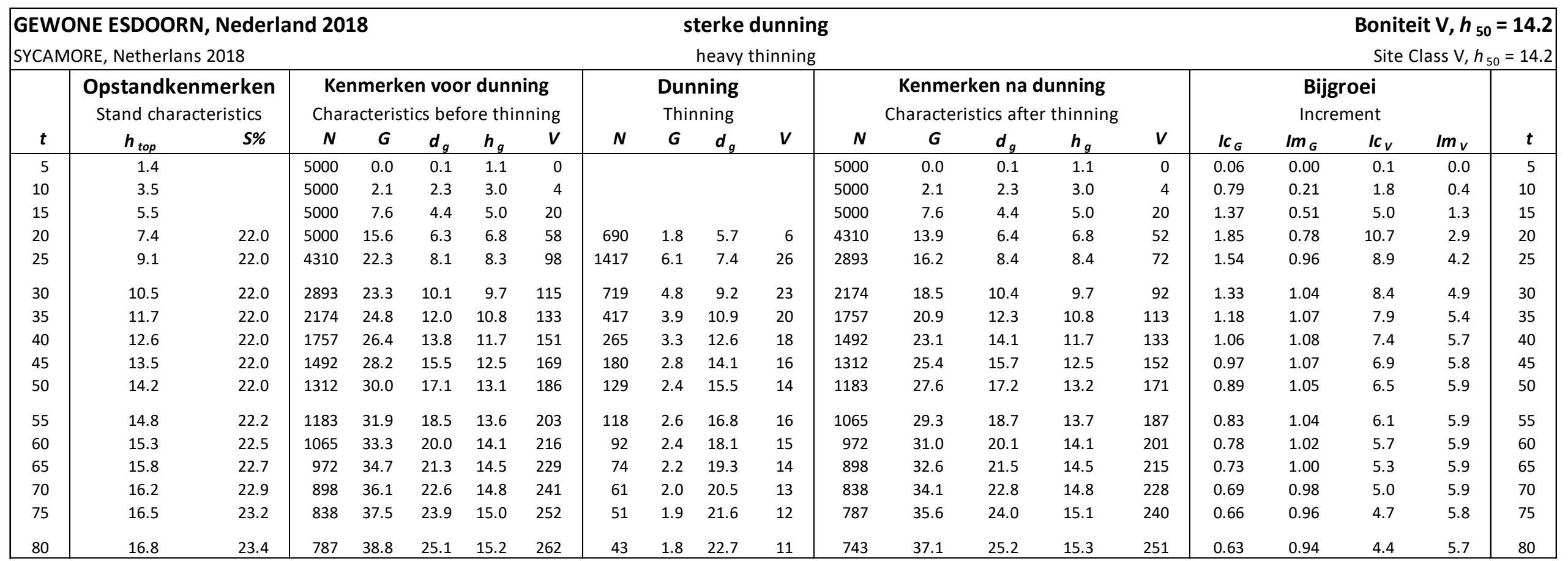

